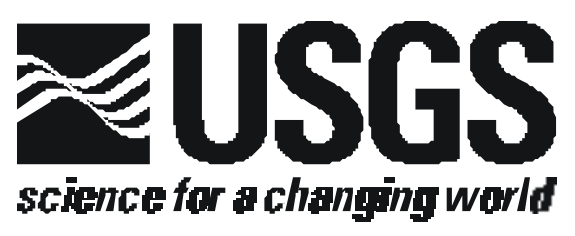

\title{
MAGNETOTELLURIC DATA ACROSS THE BATTLE MOUNTAIN-EUREKA AND CARLIN TRENDS, NEAR BATTLE MOUNTAIN, NEVADA
}

\author{
by \\ Jackie M. Williams, Brian D. Rodriguez, \\ and \\ Douglas P. Klein1
}

Open-File Report 01-228

On-line edition

2001

This report is preliminary and has not been reviewed for conformity with U.S. Geological Survey editorial standards and stratigraphic nomenclature. Any use of trade, product, or firm names is for descriptive purposes only and does not imply endorsement by the U.S.

Government.

U.S. DEPARTMENT OF THE INTERIOR

U.S. GEOLOGICAL SURVEY

1 Denver, Colorado 
TABLE OF CONTENTS

INTRODUCTION

MAGNETOTELLURIC METHOD 3

MAGNETOTELLURIC SURVEY

MAGNETOTELLURIC DATA $\quad 6$

REFERENCES CITED 9

APPENDIX - MAGNETOTELLURIC DATA PLOTS 12 


\title{
INTRODUCTION
}

\author{
Many Sediment-hosted gold deposits occur along linear \\ trends in northern Nevada. The distribution and genesis of \\ these deposits along the Battle Mountain-Eureka and Carlin \\ gold trends is not fully understood. In general, most models \\ agree that regional structures played an important role in the \\ spatial distribution of these deposits (e.g. Arehart and \\ others, 1993; Ilchik and Barton, 1997; Radtke, 1985; Shawe, \\ 1991; Sillitoe and Bonham, 1990; Tosdal, 1998). To \\ investigate crustal structures that may be related to the \\ genesis of gold deposits along these trends, a regional \\ southwest-northeast profile of magnetotelluric (MT) soundings \\ was acquired in 1996, 1997, 1999, and 2000 (1ine MT4-MT4", \\ Figure 1). Resistivity modeling of the MT data can be used to \\ infer the deep resistivity structure of the crust to help \\ investigate possible tectonic controls on the emplacement of \\ mineral deposits along these linear trends. The purpose of \\ this report is to release the MT sounding data, and no \\ interpretation of the data is included.
}

\section{MAGNETOTELLURIC METHOD}

The magnetotelluric (MT) method is a passive surface geophysical technique, which uses the earth's natural electromagnetic fields to investigate the electrical resistivity structure of the subsurface. The resistivity of geologic units is largely dependent upon their fluid content, porosity, degree of fracturing, temperature, and conductive mineral content (Keller, 1989). Saline fluids within the pore spaces and fracture openings can reduce resistivities in a resistive rock matrix. Also, resistivity can be lowered by the presence of conductive clay minerals, carbon, and metallic mineralization. It is common for altered volcanic rocks to contain authigenic minerals that have resistivities ten times lower than those of the surrounding rocks (Nelson and Anderson, 1992). Increased temperatures cause higher ionic mobility and mineral activation energy, reducing rock resistivities significantly. Unaltered, unfractured igneous rocks are normally very resistive (typically 1,000 ohm-m or greater), whereas fault zones will show low resistivity (less than $100 \mathrm{ohm-m)}$ when they are comprised of rocks fractured enough to have hosted fluid transport and consequent mineralogical alteration (Eberhart-Phillips and others, 1995). Carbonate rocks are moderately to highly resistive (hundreds 
to thousands of ohm-m) dependent upon their fluid content, porosity, fracturing, and impurities. Marine shales, mudstones, and clay-rich alluvium are normally very conductive (a few ohm-m to tens of ohm-m). Unaltered, metamorphic rocks (non-graphitic) are moderately to highly resistive (hundreds to thousands of ohm-m). Tables of electrical resistivity for a variety of rocks, minerals and geological environments may be found in Keller (1987) and Palacky (1987).

The MT method can be used to probe the crust from depths of tens of meters to depths of tens of kilometers (Vozoff, 1991). Natural variations of the Earth's magnetic and electric field are measured and recorded at each MT station. The main frequency bands used by the MT method are $10,000 \mathrm{~Hz}$ to $1 \mathrm{~Hz}$ from worldwide lightning activity and $1 \mathrm{~Hz}$ to 0.0001 $\mathrm{Hz}$ from geomagnetic micro-pulsations. The natural electric and magnetic fields propagate vertically in the earth because the very large resistivity contrast between the air and the earth causes a vertical refraction of both fields transmitted into the earth (Vozoff, 1972).

The natural electric and magnetic fields are recorded in two orthogonal, horizontal directions. The vertical magnetic field ("tipper") is also recorded. The resulting time-series signals are used to derive earth tensor apparent resistivities and phases by first converting them to complex cross-spectra using FFT (fast-Fourier-transform) techniques. Least-squares, cross-spectral analysis (Bendat and Piersol, 1971) is used to solve for a tensor-transfer function that relates the observed electric fields to the magnetic fields under the assumption that the Earth consists of a two-input, two-output, linear system with the magnetic fields as input and the electric fields as output (Rodriguez and others, 1996). Prior to conversion to apparent resistivity and phase, the tensor is normally rotated into principal directions that correspond to the direction of maximum and minimum apparent resistivity. For a two-dimensional (2-D) Earth, the MT fields can be decoupled into transverse electric (TE) and transverse magnetic (TM) modes; 2-D modeling is generally done to fit both modes. When the geology satisfies the 2-D assumption, the MT data for the TE mode is assumed to represent the situation when the electric field is along the geologic strike, and the data for the TM mode is assumed to represent the situation when the electric field is across strike. The MT method is well suited for studying complicated geological environments because the electric and magnetic relations are sensitive to vertical and horizontal variations in resistivity. The method is capable of establishing whether the electromagnetic fields are 
responding to subsurface terranes of effectively 1-, 2-, or 3dimensions. An introduction to the MT method and references for a more advanced understanding are contained in Dobrin and Savit (1988) and Vozoff (1991).

\section{MAGNETOTELLURIC SURVEY}

Twenty-three MT soundings were located along or near profile MT4-MT4" (Figure 1) with spacing that varied from 2 to 13.5 kilometers. The profile orientation is roughly perpendicular to the Battle Mountain-Eureka and Carlin trends. Only stations 24B-28B were collected with the U.S. Geological Survey's truck-mounted MT system (Stanley, 1978). All other stations were collected with a portable EMI MT-1 system (EMI, 1996). Horizontal electric fields were sensed using an Lshaped, three-electrode array with dipole lengths of $30 \mathrm{~m}$ except for stations 24B-28B where a dipole length of $37.5 \mathrm{~m}$ was used with the truck-mounted MT system. The orthogonal, horizontal magnetic fields in the direction of the electricfield measurement array were sensed using permalloy-cored induction coils (Stanley and Tinkler, 1983). Frequencies sampled ranged from 200 to $0.002 \mathrm{~Hz}$ using single station recordings of both orthogonal horizontal components of the electric and magnetic fields, along with the vertical magnetic field at all stations except 24B-28B. Sampling this frequency range in previous areas of widely varying geology has allowed us to probe the crust from depths of hundreds of meters to depths of tens of kilometers.

The recorded time-series data were transformed to the frequency domain and Fourier analyzed to determine a twodimensional apparent resistivity and phase tensor at each site. The data were rotated to maximum and minimum apparent resistivity directions so that propagation modes for the signals were decoupled into TE and TM modes. Local reference sensors to help reduce bias in the impedance determinations due to instrument or environmental noise (Gamble and others, 1979a; Clarke and others, 1983) were used at all stations except 24B-28B and 98. Although true remote reference techniques were not used in our survey, we did sort crosspower files to select optimal signal-to-noise data sets (see Appendix).

The effects of near-surface resistivity anomalies cause "static shifts" (Sternberg and others, 1988) in the data. Static shifts of this data set ranged from 0.0 to 0.8 of a $10 \mathrm{~g}$ 
decade. Only stations 14, 23, 25A, 26A, and 27B had static shifts of 0.4 of a log decade or greater. The remainder of the stations had an average of 0.2 of a log decade static shift.

\section{MAGNETOTELLURIC DATA}

The following table shows twenty-three magnetotelluric (MT) station locations (from southwest to northeast). Coordinates are referenced to the 1866 Clarke spheroid and North American 1927 Western United States datum. Longitude and latitude format below is decimal degrees. Elevation is in meters.

\begin{tabular}{|c|c|c|c|}
\hline Station & Longitude & Latitude & Elev (m) \\
\hline 98 & $\overline{-117.23117}$ & $\overline{40.44410}$ & 1440 \\
\hline 12 & -117.12441 & 40.44999 & 1430 \\
\hline 13 & -117.05447 & 40.46508 & 1420 \\
\hline 14 & -116.98314 & 40.49695 & 1390 \\
\hline 15 & -116.95895 & 40.47327 & 1430 \\
\hline 16 & -116.94524 & 40.51559 & 1360 \\
\hline 19 & -116.89245 & 40.51839 & 1390 \\
\hline 20 & -116.86685 & 40.52878 & 1350 \\
\hline 22 & -116.84852 & 40.55662 & 1340 \\
\hline 23 & -116.82223 & 40.56811 & 1350 \\
\hline 21 & -116.80062 & 40.53557 & 1400 \\
\hline $24 \mathrm{~A}$ & -116.79907 & 40.58977 & 1340 \\
\hline $25 \mathrm{~A}$ & -116.78090 & 40.61226 & 1400 \\
\hline 71 & -116.76773 & 40.69609 & 1350 \\
\hline $26 \mathrm{~A}$ & -116.70164 & 40.66617 & 1450 \\
\hline $27 A$ & -116.54826 & 40.73778 & 1410 \\
\hline $28 \mathrm{~A}$ & -116.44901 & 40.78003 & 1420 \\
\hline 1 & -116.33037 & 40.79034 & 1550 \\
\hline $24 B$ & -116.29118 & 40.79805 & 1650 \\
\hline $25 B$ & -116.25092 & 40.79475 & 1770 \\
\hline $26 B$ & -116.22477 & 40.82100 & 1600 \\
\hline $27 B$ & -116.18810 & 40.80681 & 1600 \\
\hline $28 B$ & -116.15437 & 40.80115 & 1600 \\
\hline
\end{tabular}

The figures in the Appendix represent the field-processed MT data for each station after the time series data were converted to the frequency domain and the tensor-transfer function was rotated into principal directions as described above in the "Magnetotelluric Method" section. 
For each station, nine separate graph plots are given:

1. Apparent Resistivity for the rotated maximum ( $x$ symbol) and minimum (o symbol) modes

2. Impedance Phase for the rotated maximum ( $x$ symbol) and minimum (o symbol) modes

3. Rotation Angle for the impedance tensor (corresponds to the direction of maximum apparent resistivity)

4. Impedance Skew for the impedance tensor

5. Multiple Coherency for the rotated maximum ( $x$ symbol) and minimum (o symbol) modes of the electric field

6. Impedance Polar Plots (at 12 selected frequencies)

7. Tipper Magnitude for the vertical magnetic field

8. Tipper strike for the vertical magnetic field, and

9. HzHx (x symbol) and HzHy (o symbol) Coherency

Error bars that appear on the Apparent Resistivity, Impedance Phase, Skew, Tipper Magnitude, and Tipper Strike plots represent probable errors within one standard deviation of the sample variance (Gamble and others, 1979b).

Apparent resistivity is a measure of the magnitude of the electric field strength over the magnetic field strength for a given frequency. The impedance phase is proportional to the slope of the apparent resistivity curve on a log-log scale, but from a baseline at -45 degrees (Vozoff, 1991). A measure of the dimensionality for MT data is provided by the impedance skew of the impedance tensor (Vozoff, 1972). If the effective measured resistivity response to the geology beneath a MT station is truly $1-D$ or $2-D$, then the skew will be zero. Both instrument and environmental sources of noise contribute to non-zero skew values, but are typically small (about 0.1) for relatively low noise level recordings. Higher skews (above 0.2) are an indication of either 3-D resistivity responses to the geology or higher levels of noise. Man-made electrical noise, such as power lines, power generators, moving vehicles and trains can have a negative effect on $\mathrm{MT}$ data quality. All these local disturbances produce an incoherent noise mainly affecting frequencies above $1 \mathrm{~Hz}$. other man-made electrical noise, such as direct current electric trains and active cathodic protection of pipelines produce coherent electromagnetic signals mainly affecting frequencies below $1 \mathrm{~Hz}$.

In the survey area, noise from a number of small power lines and small moving vehicles was negligible at distances of $0.4 \mathrm{~km}$ and greater from the noise source. Power line levels 
were measured at each site and were typically less than 20\% of the maximum recordable signals. Noise from larger power lines, power generators, pipelines, railroads, and steamdriven trains, mostly near mining operations, was negligible at least $5 \mathrm{~km}$ from them. Recordings were not made when noise from moving vehicles affected the magnetic signals. Local lightning, wind, and rainstorms can also degrade data quality, but these were avoided by not recording during active thunderstorm periods. Wind noise was minimized by burying the magnetic induction coils.

Predicted values of the electric field can be computed from the measured values of the magnetic field (Vozoff, 1991). The coherence of the predicted electric field with the measured electric field is a measure of the signal-to-noise ratio provided in the multiple coherency plot. Values are normalized between 0 and 1 , where values at 0.5 signify signal levels equal to noise levels. For this data set, coherencies were generally at an acceptable level, except at times in the "dead band" (0.1 to $1 \mathrm{~Hz})$ and at times in the lower frequencies $(0.002$ to 0.1$)$. The lower frequency ionospheric signals are related to sunspot activity whose levels typically follow an 11-year cycle. The sunspot activity was near the lowest level of the cycle during the 1996 and 1997 surveys, and was near the highest level in the 1999 and 2000 surveys.

The figures in the Appendix represent the field-processed MT data at each station, which includes some data scatter and poor signal-to-noise ratios. Our only effort at removing noisy data points was to visually inspect and select the best signal-to-noise field data to combine into the final data plots.

The impedance polar plots provide a measure of the MT data dimensionality (Reddy and others, 1977). For 1-D resistivity structures, the principal impedance polar diagram (dashed line) is a circle. For 2-D or 3-D resistivity structures, the principal impedance polar diagram (dashed line) elongates either parallel or perpendicular to strike direction. Over resistors, the principal impedance polar diagram elongates perpendicular to strike direction and over conductors, the principal impedance polar diagram elongates parallel to strike direction. Also, for 2-D resistivity structures, the additional impedance polar diagram (solid line) attains the shape of a symmetric clover leaf. For 3-D resistivity structures, the additional impedance polar diagram (solid line) elongates in one direction and its amplitude is comparable to that of the principal impedance polar diagram 
(dashed line). Sites whose polar plots indicated 3-D character in the lower frequencies were MT stations 98, 13, 14, 19-21, 71, 26A-28A, 1, 24B, 26B, and 27B (Figure 1).

The tipper can be solved for when the vertical component of the magnetic field is measured. The tipper magnitude is a measure of the tipping of the magnetic field out of the horizontal plane (Vozoff, 1991). The magnitude is zero for the 1-D case and typically increases between 0.1 to 0.5 , and rarely as great as 1 , as it responds to vertical and subvertical structures. The tipper strike is typically used to help resolve the 90-degree ambiguity in the impedance rotation angle. The vertical component of the magnetic field was measured at all MT stations except 24B-28B. The tipper magnitude of these stations was typically 0.1 to 0.4 over the lower frequencies indicating vertical structure at depth. Stations 13, 25A, and 26A had even larger tipper magnitudes (over 0.5 ) in the lower frquencies indicating nearby vertical structure at depth. The $\mathrm{HzHx}$ and $\mathrm{HzHy}$ coherency is a measure of the signal-to-noise ratio of the vertical magnetic field with respect to each of the orthogonal horizontal magnetic field directions. Values are normalized between 0 and 1, where values at 0.5 signify signal levels equal to noise levels. These three-component magnetic field coherencies provide a check on the signal-to-noise ratio of the measured values in the tipper magnitude and tipper strike plots.

\section{REFERENCES CITED}

Arehart, G.B., Foland, K.A., Naeser, C.W., and Kesler, S.E., 1993, 40Ar/39Ar, K/Ar, and fission track geochronology of sediment-hosted disseminated gold deposits at Post-Betze, Carlin Trend, northeastern Nevada: Economic Geology, vol. 88 , no. 3, p. $622-646$.

Bendat, J.S., and Piersol, A.G., 1971, Random Data: Analysis and Measurement Procedures: New York, Wiley Interscience, $407 \mathrm{p}$.

Clarke, J., Gamble, T.D., Goubau, W.M., Koch, R.H., and Miracky, R.F., 1983, Remote-reference magnetotellurics: Equipment and procedures: Geophysical Prospecting, vol. $31, \mathrm{p}, 149-170$. 
Dobrin, M.D., and Savit, C.H., 1988, Introduction to Geophysical Prospecting (4th ed.): New York, McGraw-Hill, $867 \mathrm{p}$.

Eberhart-Phillips, D., Stanley, W. D., Rodriguez, B. D. and Lutter, W. J., 1995, Surface seismic and electrical methods to detect fluids related to faulting: Journal of Geophysical Research, vol. 100, no. B7, pp. 12,91912,936 .

EMI, 1996, MT-1 magnetotelluric system operation manual, version 3.2: ElectroMagnetic Instruments, Inc., Richmond, California, $220 \mathrm{p}$.

Gamble, T.D., Goubau, W.M. and Clarke, J., 1979a, Magnetotellurics with a remote magnetic reference: Geophysics, v. 44, no. 1, p. 53-68.

Gamble, T.D., Goubau, W.M. and Clarke, J., 1979b, Error analysis for remote reference magnetotellurics: Geophysics, v. 44, no. 5, p. 959-968.

Ilchik, R.P. and Barton, M.D., 1997, An amagmatic origin of Carlin-type gold deposits: Economic Geology, vol. 92 , no. 3 , p. 269-288.

Keller, G.V., 1987, Rock and mineral properties, in Electromagnetic Methods in Applied Geophysics Theory: M.N. Nabighian, Ed., Society of Exploration Geophysicists, Tulsa, Oklahoma, v. 1, p. 1351.

Keller, G.V., 1989, Electrical properties, in Carmichael, R.S., Ed., Practical handbook of physical properties of rocks and minerals: CRC Press, Boca Raton, Florida, p. $359-427$.

Nelson, P.H. and Anderson, L.A., 1992, Physical properties of ash flow tuff from Yucca Mountain, Nevada: Journal of Geophysical Research, vol. 97, no. B5, p. 6823-6841.

Palacky, G.J., 1987, Resistivity characteristics of geologic targets, in Electromagnetic Methods in Applied Geophysics Theory: M.N. Nabighian, Ed., Society of Exploration Geophysicists, Tulsa, Oklahoma, vol. 1, p. 53129. 
Radtke, A.S., 1985, Geology of the Carlin gold deposit, Nevada: U.S. Geological Survey Professional Paper 1267, $124 \mathrm{p}$.

Reddy, I.K., Rankin, D., and Phillips, R.J., 1977, Threedimensional modeliing in magnetotelluric and magnetic variational sounding: Geophysics Journal of the Royal Astronomical Society, vol. 51, p. 313-325.

Rodriguez, B.D., Stanley, W.D., and Williams, J.M., 1996, Axial structures within the Reelfoot rift delineated with magnetotelluric surveys: U.S. Geological Survey Professional Paper $1538-\mathrm{K}, 30 \mathrm{p}$.

Shawe, D.R., 1991, Structurally controlled gold trends imply large gold resources in Nevada, in Geology and ore deposits of the Great Basin, Symposium Proceedings: Raines, G.L., Lisle, R.E., Schafe, R.W., Wilkinson, W.H., Eds., Geological Society of Nevada, Reno, vol. 1, p. 199212 .

Sillitoe, R.H. and Bonham, H.F., 1990, Sediment-hosted gold deposits; distal products of magmatic-hydrothermal systems: Geology, vol. 18, no. 2, p. 157-161.

Stanley, W.D., 1978, New magnetotelluric field processing system: U.S. Geological Survey Professional Paper 1100, $174 \mathrm{p}$.

Stanley, W.D., and Tinkler, R.D., 1983, A practical, low-noise coil system for magnetotellurics: U.S. Geological Survey Open-File Report 83-85, $18 \mathrm{p}$.

Sternberg, B.K., Washburne, J.C., and Pellerin, L., 1988, Correction for the static shift in magnetotellurics using transient electromagnetic soundings: Geophysics, vol. 53, p. $1459-1468$.

Struhsacker, E.M., Jones, E., and Green, S.M., 1996, Roadside geology and precious-metal mineralization along the I-80 corridor, Reno to Elko, Nevada, in Struhsacker, E.M. and Green, S.M., eds., Geology and ore deposits of the American Cordillera - Field Trip Guidebook Compendium: Geological Society of Nevada, Reno, Nevada, p. 3. 
Tosdal, R.M., 1998, Contributions to the gold metallogeny of northern Nevada: U.S. Geological Survey Open-File Report $98-338,290 \mathrm{p}$.

Vozoff, K., 1972, The magnetotelluric method in the exploration of sedimentary basins: Geophysics, vol. 37, p. $98-141$.

Vozoff, K., 1991, The magnetotelluric method, in Electromagnetic methods in applied geophysics: M.N. Nabighian, Ed., Society of Exploration Geophysicists, Tulsa, Oklahoma, vol. 2, part B, p. 641-711.

\section{APPENDIX}

\section{MAGNETOTELLURIC DATA PLOTS}

For all stations except 24B-28B, there are nine separate graph plots:

1. Apparent Resistivity for the rotated maximum (x symbol) and minimum (o symbol) modes

2. Impedance Phase for the rotated maximum ( $x$ symbol) and minimum (o symbol) modes

3. Rotation Angle for the impedance tensor (corresponds to the direction of maximum apparent resistivity)

4. Impedance skew for the impedance tensor

5. Multiple Coherency for the rotated maximum (x symbol) and minimum (o symbol) modes of the electric field

6. Impedance Polar Plots (at 12 selected frequencies)

7. Tipper Magnitude for the vertical magnetic field

8. Tipper strike for the vertical magnetic field, and

9. $\mathrm{HzHx}$ ( $x$ symbol) and $\mathrm{HzHy}$ (o symbol) Coherency

Stations 24B-28B have only the first six graph plots above, since the vertical magnetic field data (Tipper, Hz) was not acquired. Refer to the "Magnetotelluric Data" section in this report for an explanation of these plots. 


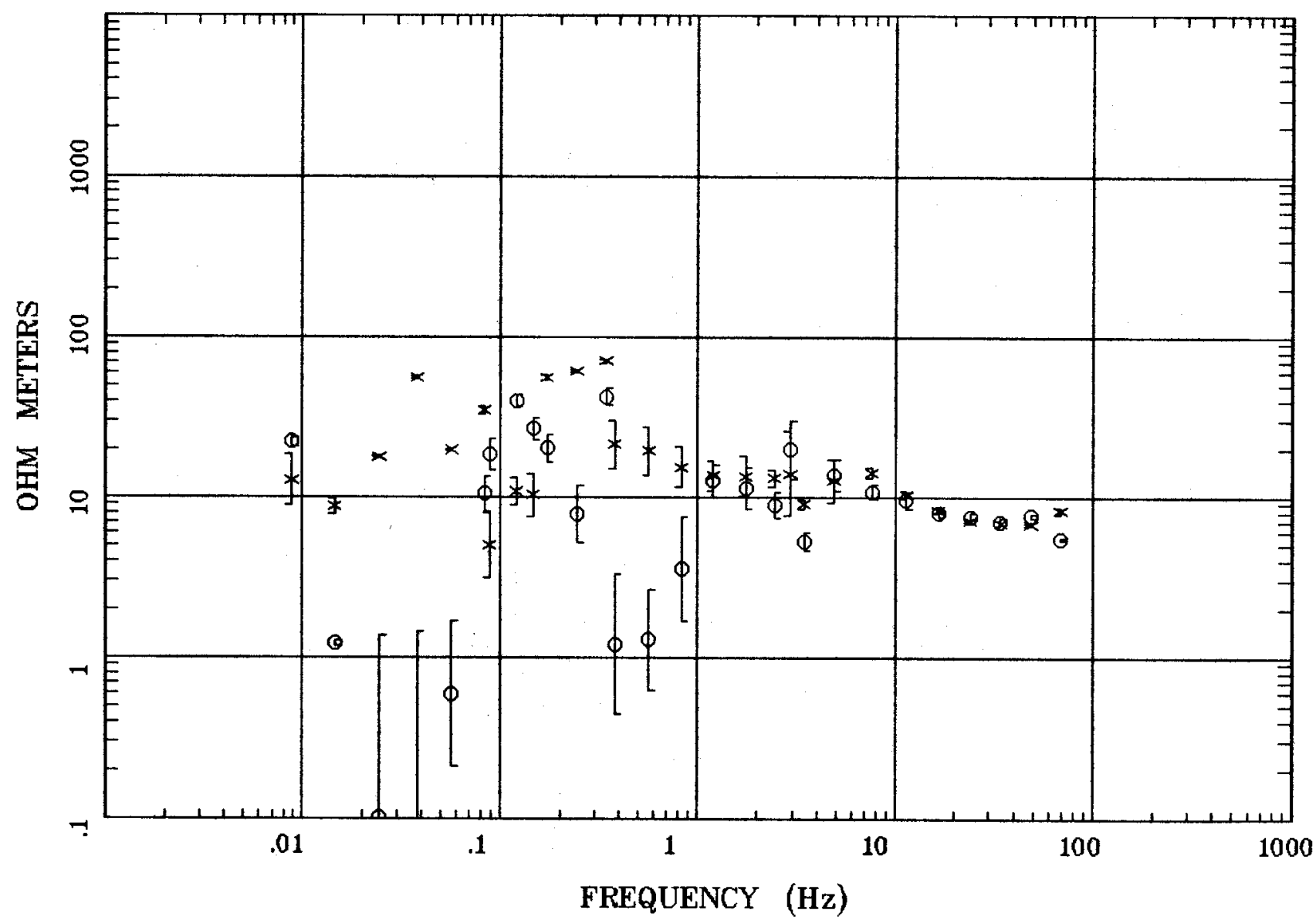

Client:

Remote: none

Acquired: 13:1 Jul 30, 2000

Survey Co:USGS
Rotation:

Filename: hr98c.avg

Channels: Ch1 Ch2 Ch3 Ch4 Ch5 Ch3 Ch4

Plotted: 08:24 Dec 08, 2000

< EMI - ElectroMagnetic Instruments > 
Fish Creek Mtns, NV 100k

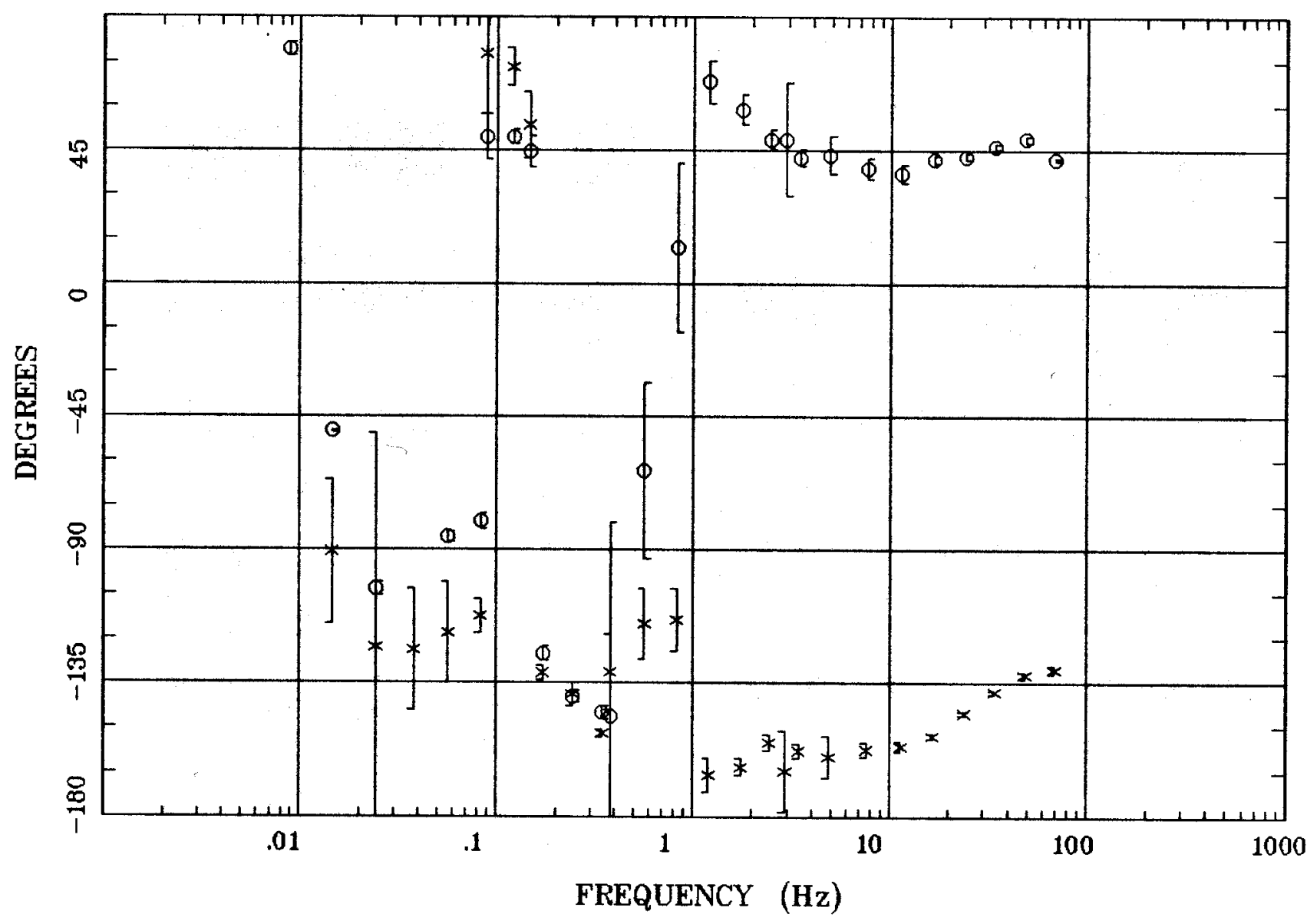

Client:

Remote: none

Acquired: 13:1 Jul 30, 2000

Survey Co:USGS
Rotation:

Filename: hr98c.avg

Channels: Ch1 Ch2 Ch3 Ch4 Ch5 Ch3 Ch4

Plotted: 08:24 Dec 08, 2000

< EMI - ElectroMagnetic Instruments 
Fish Creek Mtns, NV 100k

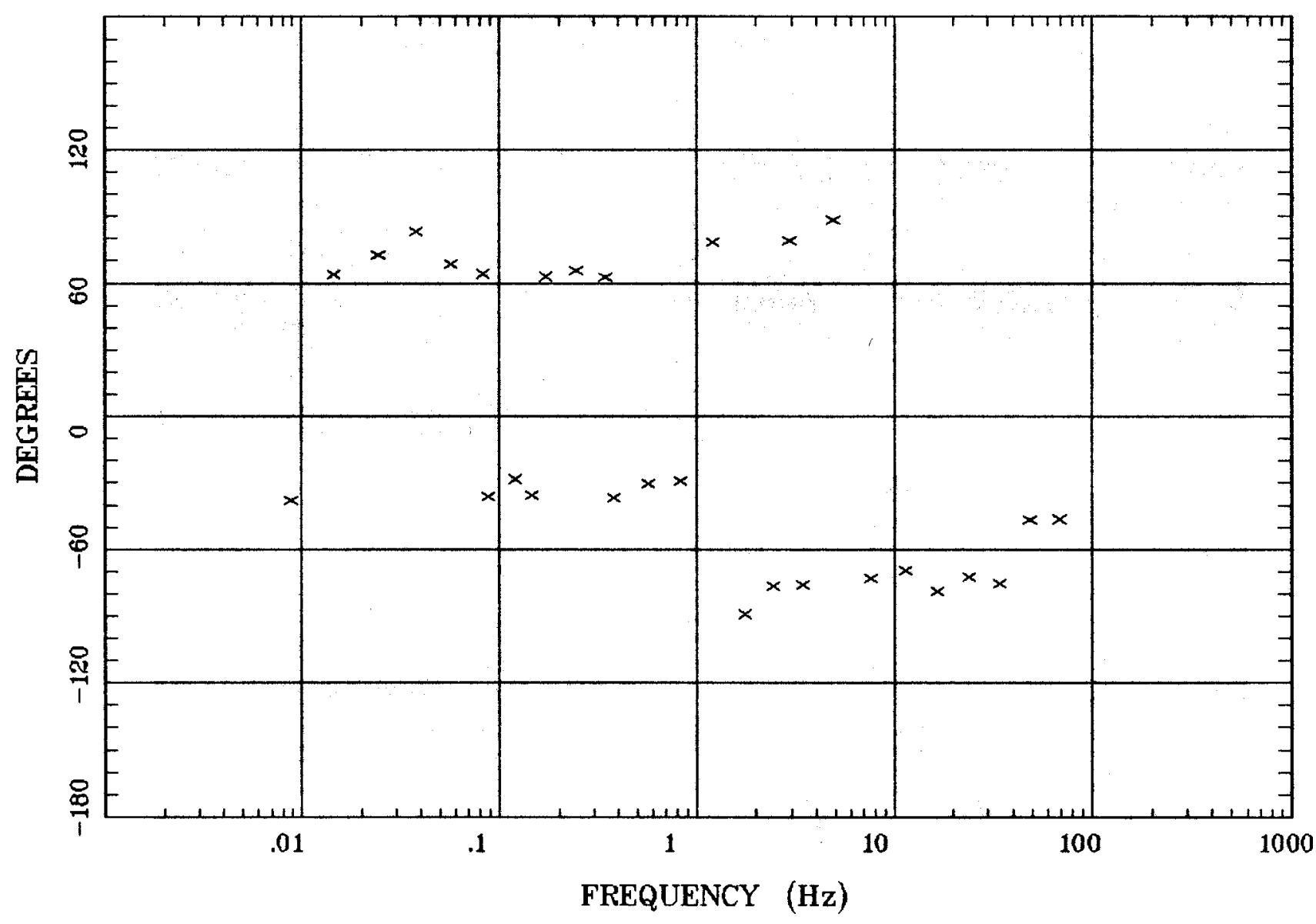

Client:

Remote: none

Acquired: $13: 1$ Jul 30, 2000 Survey Co:USGS
Rotation:

Filename: hr98c.avg

Channels: Ch1 Ch2 Ch3 Ch4 Ch5 Ch3 Ch4 Plotted: 08:24 Dec 08, 2000

$<$ EMI - ElectroMagnetic Instruments 


\section{Station 98}

IMPEDANCE SKEW

Fish Creek Mtns, NV 100k

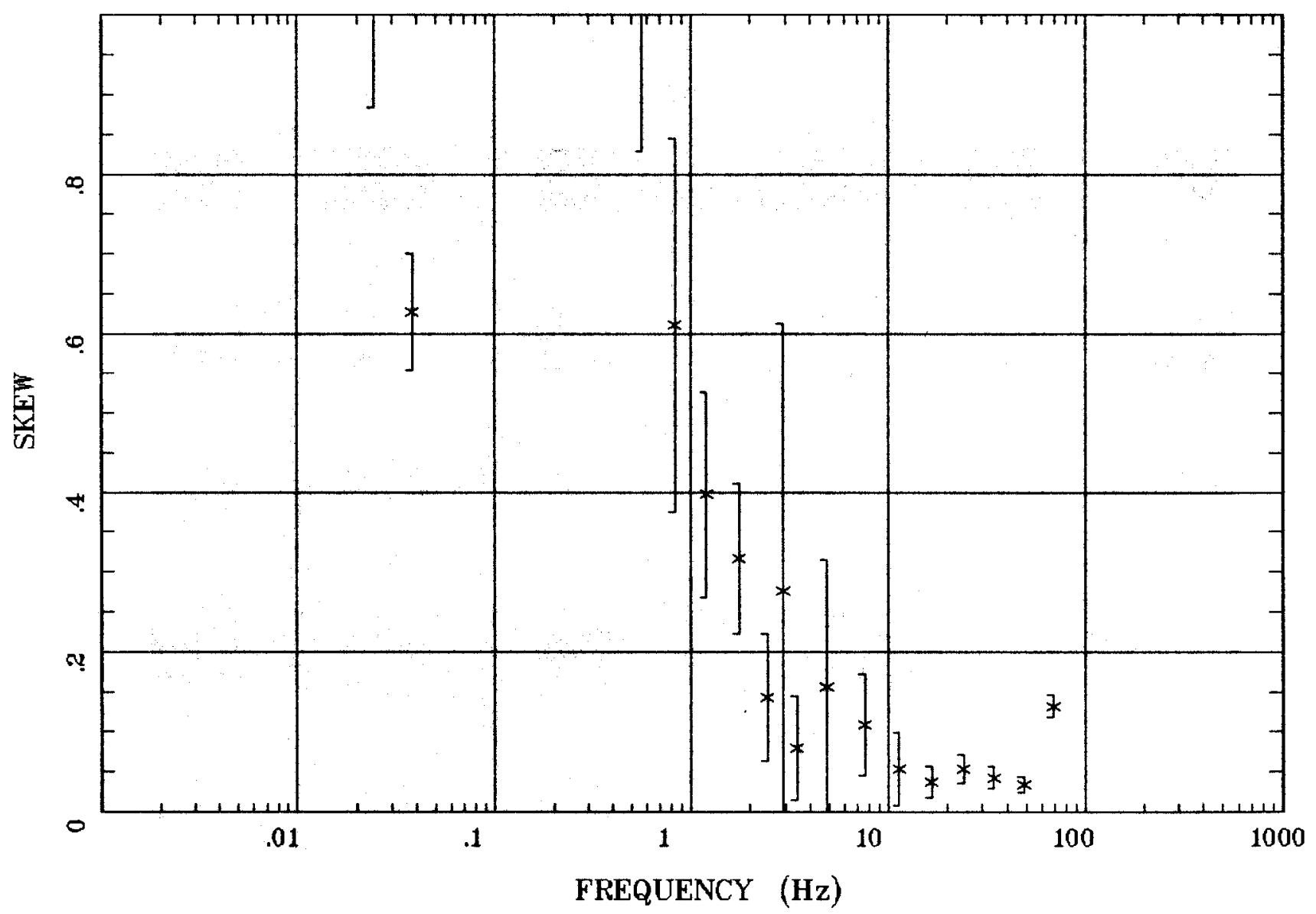

Client:

Remote: none

Acquired: 13:1 Jul 30, 2000

Survey Co:USGS
Rotation:

Filename: hr98c.avg

Channels: Ch1 Ch2 Ch3 Ch4 Ch5 Ch3 Ch4

Plotted: 08:24 Dec 08, 2000

$<$ EMI - ElectroMagnetic Instruments 
Station 98

E MULT Coh.

Fish Creek Mtns, NV 100k

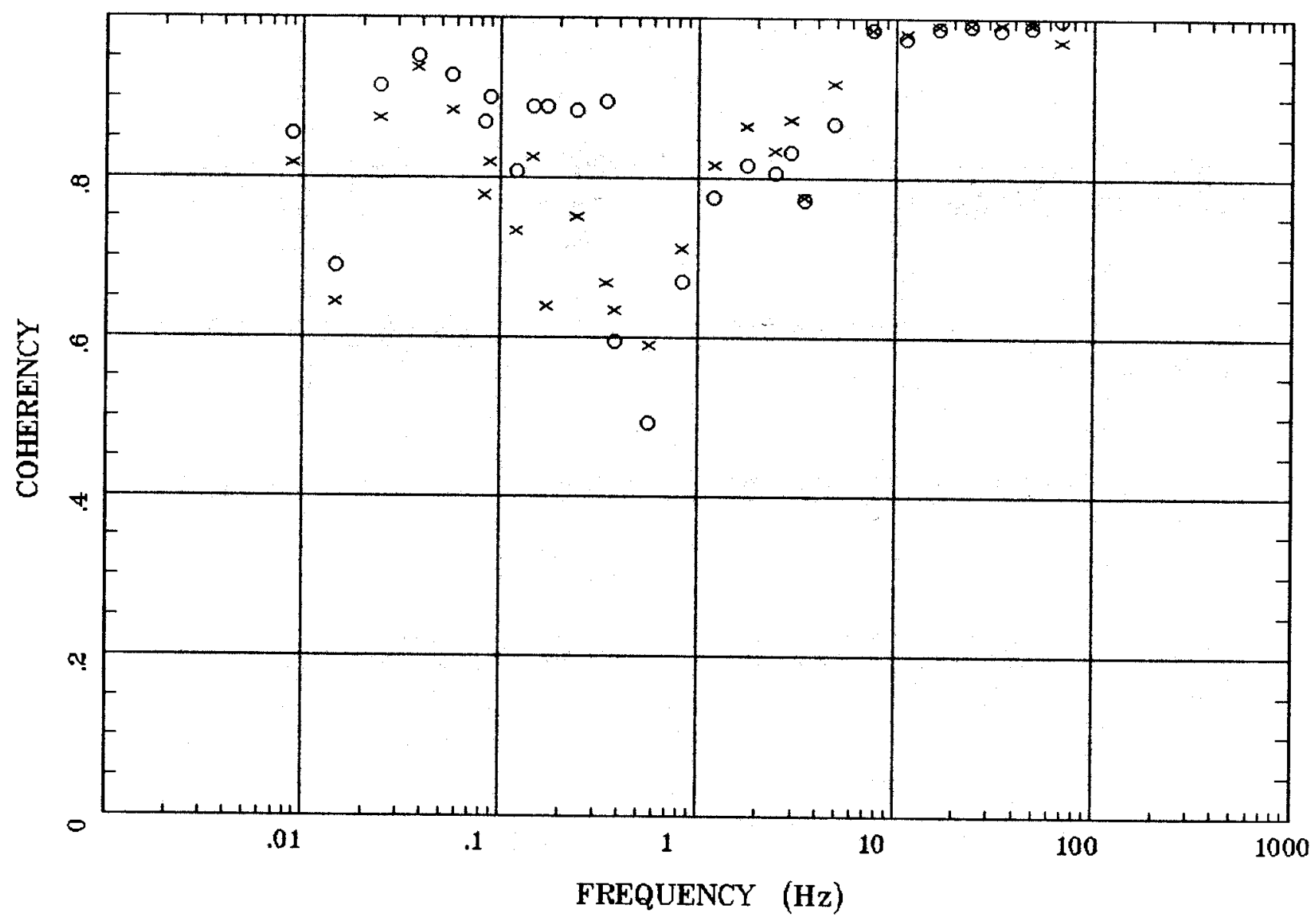

Client:

Remote: none

Acquired: $13: 1$ Jul 30, 2000

Survey Co:USGS
Rotation:

Filename: hr98c.avg

Channels: Ch1 Ch2 Ch3 Ch4 Ch5 Ch3 Ch4

Plotted: 08:24 Dec 08, 2000

$<$ EMI - ElectroMagnetic Instruments 
Station 98

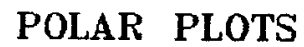

Fish Creek Mtns, NV 100k

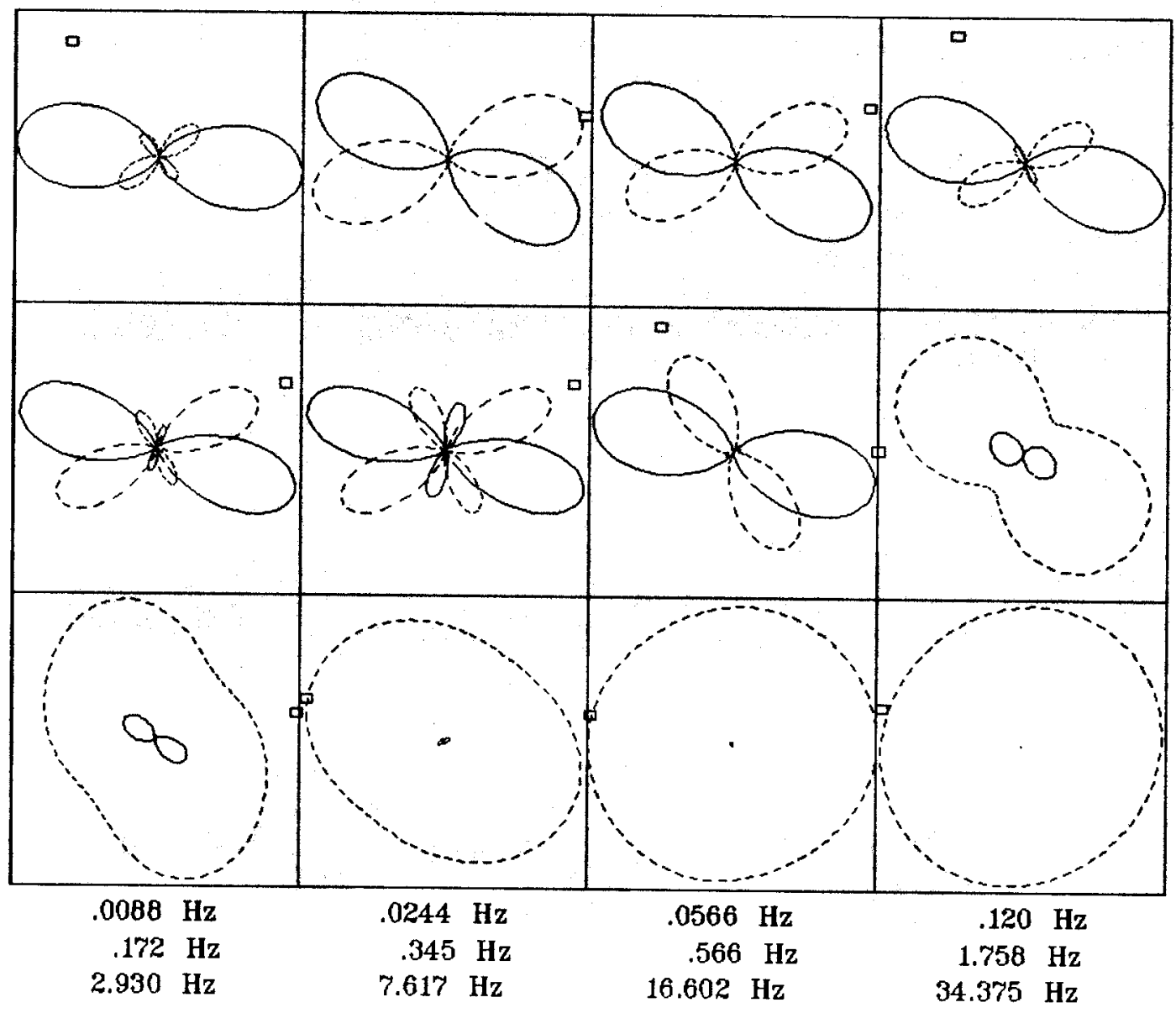

Client:

Remote: none

Acquired: 13:1 Jul 30, 2000 Survey Co:USGS
Rotation:

Filename: hr98c.avg

Channels: Ch1 Ch2 Ch3 Ch4 Ch5 Ch3 Ch4

Plotted: 08:24 Dec 08, 2000

< EMI - ElectroMagnetic Instruments > 
TIPPER MAGNITUDE

Fish Creek Mtns, NV 100k

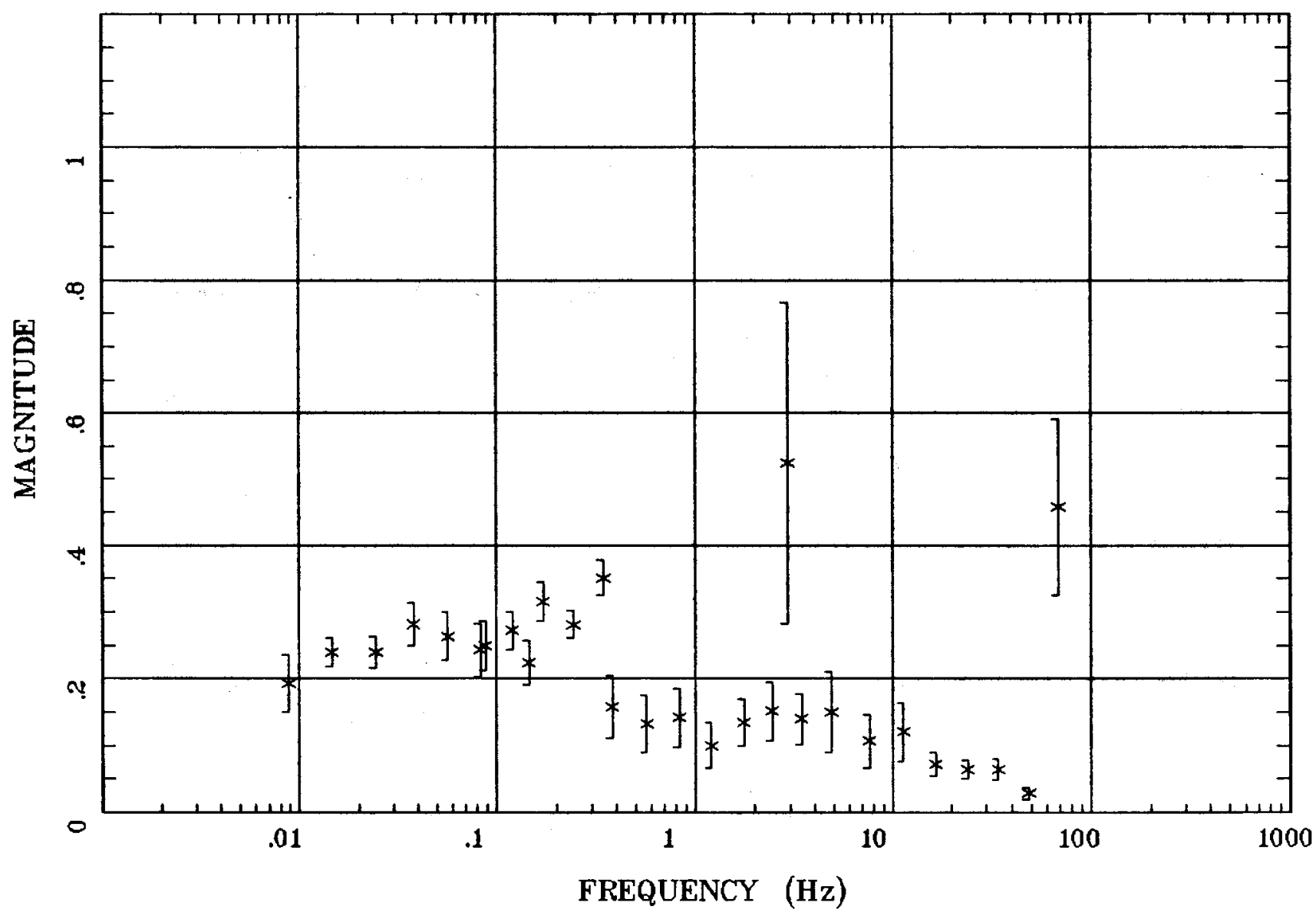

Client:

Remote: none

Acquired: $13: 1$ Jul 30, 2000 Survey Co:USGS
Rotation:

Filename: hr98c.avg

Channels: Ch1 Ch2 Ch3 Ch4 Ch5 Ch3 Ch4 Plotted: 08:24 Dec 08, 2000

< EMI - ElectroMagnetic Instruments > 
Fish Creek Mtns, NV 100k

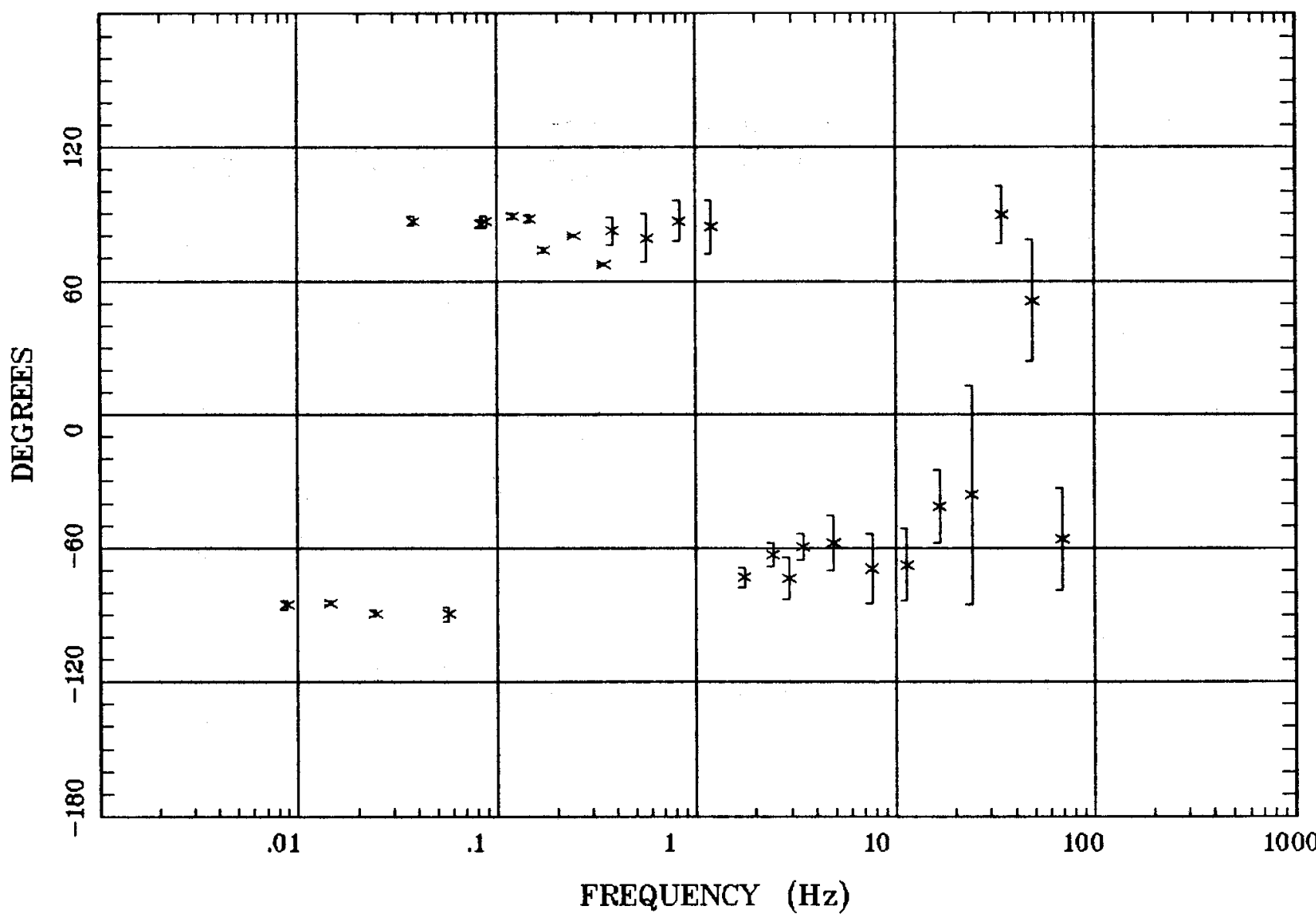

Client:

Remote: none

Acquired: 13:1 Jul 30, 2000

Survey Co:USGS
Rotation:

Filename: hr98c.avg

Channels: Ch1 Ch2 Ch3 Ch4 Ch5 Ch3 Ch4 Plotted: 08:24 Dec 08, 2000

< EMI - ElectroMagnetic Instruments 
HzHx.x Coh HzHy.o

Fish Creek Mtns, NV 100k

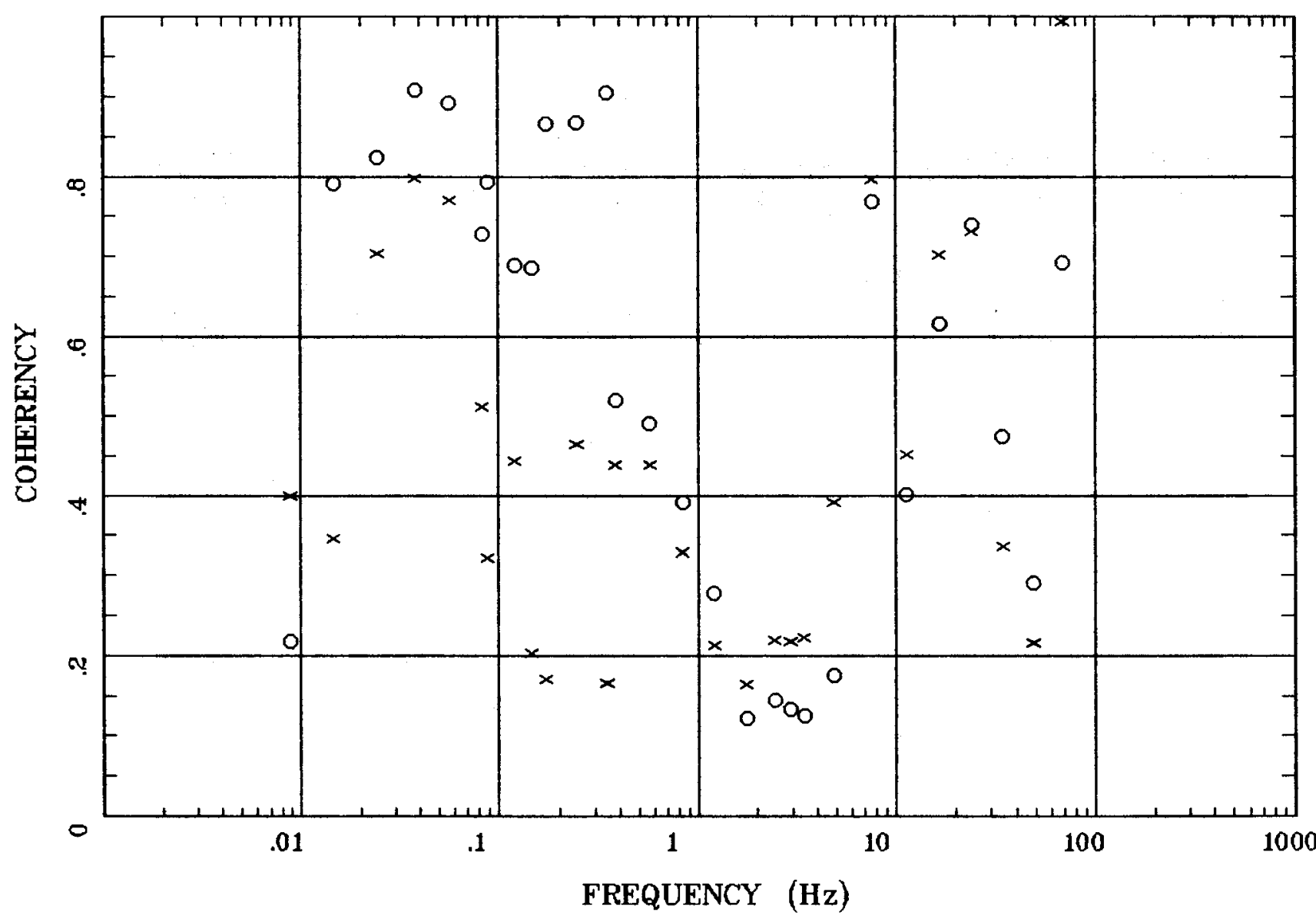

Client:

Remote: none

Acquired: 13:1 Jul 30, 2000

Survey Co:USGS
Rotation:

Filename: hr98c.avg

Channels: Ch1 Ch2 Ch3 Ch4 Ch5 Ch3 Ch4 Plotted: 08:25 Dec 08, 2000

< EMI - ElectroMagnetic Instruments 
APPARENT RESISTIVITY

battle mtn No. NV

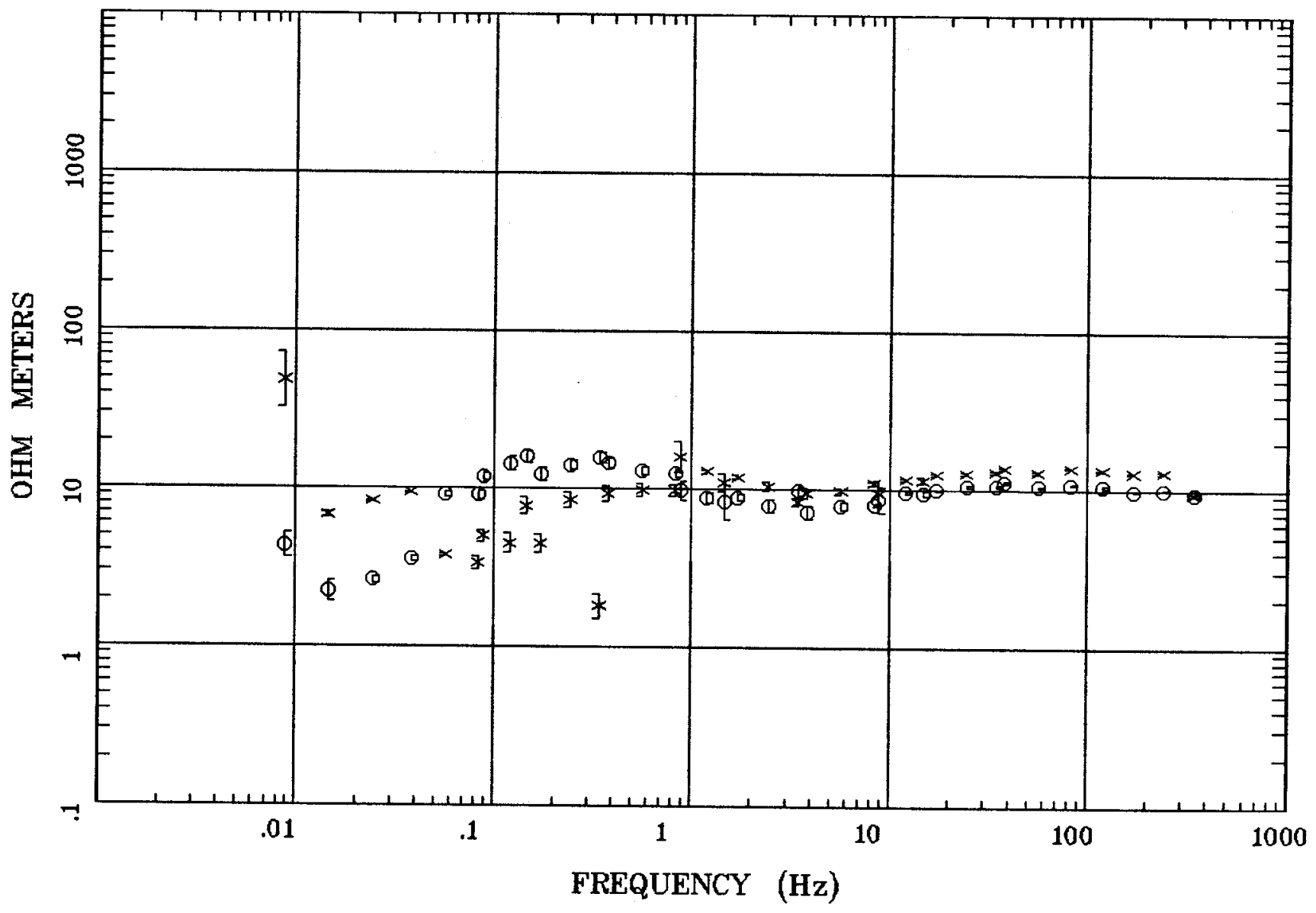

Client:

Rotation:

Remote: e-field local ref

Filename: nna12.all

Acquired: 17:0 Jul 09, 1997

Channels: Ch1 Ch2 Ch3 Ch4 Ch5 Ch6 Ch7

Plotted: 08:51 Dec 08, 2000

Survey Co:

< EMI - ElectroMagnetic Instruments 
IMPEDANCE PHASE

battle mtn No. NV

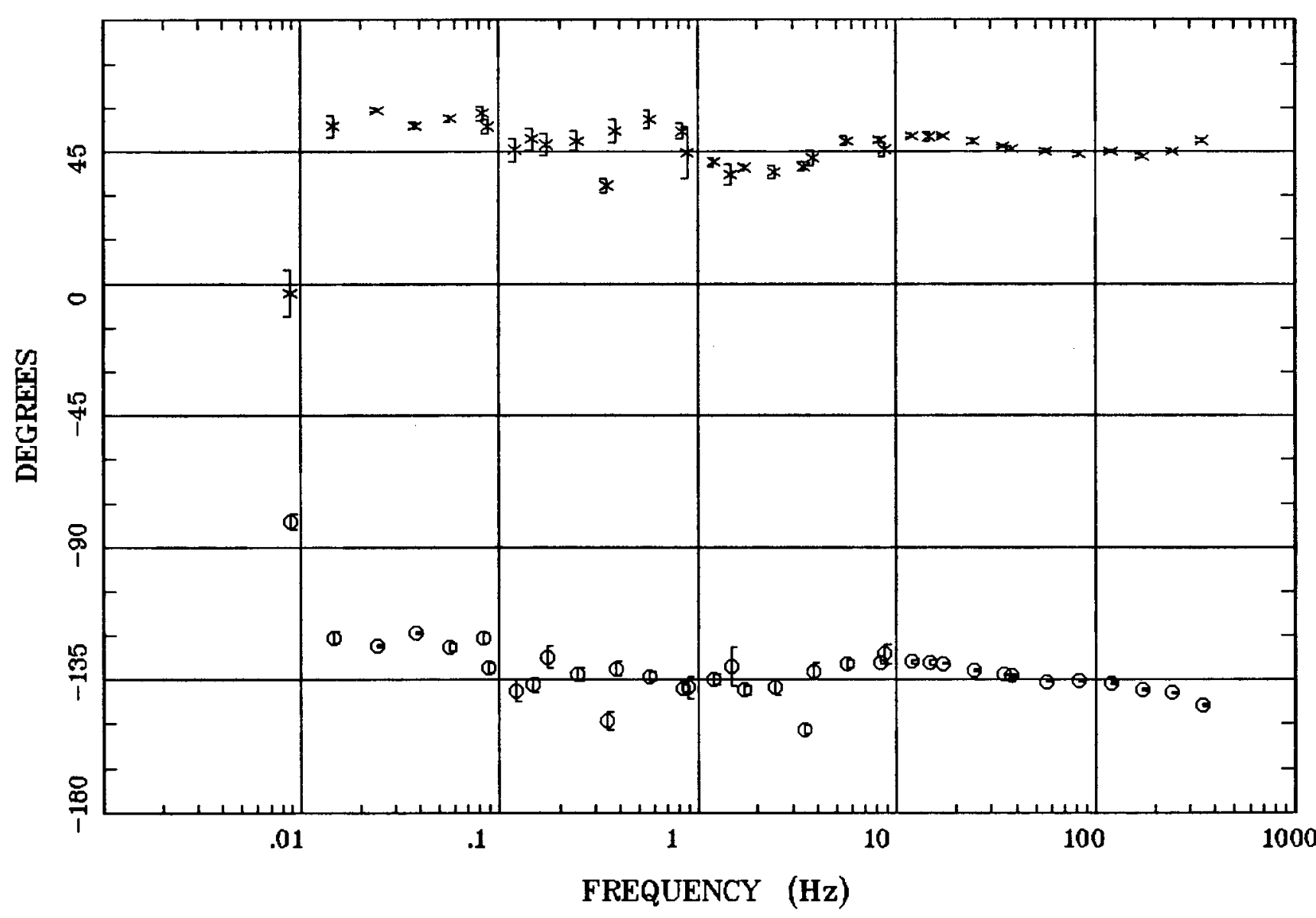

Client:

Remote: e-field local ref

Acquired: 17:0 Jul 09, 1997 Survey Co:
Rotation:

Filename: nna12.all

Channels: Ch1 Ch2 Ch3 Ch4 Ch5 Ch6 Ch7 Plotted: 08:51 Dec 08, 2000

$<$ EMI - ElectroMagnetic Instruments > 


\section{ROTATION ANGLE}

battle mtn No. NV

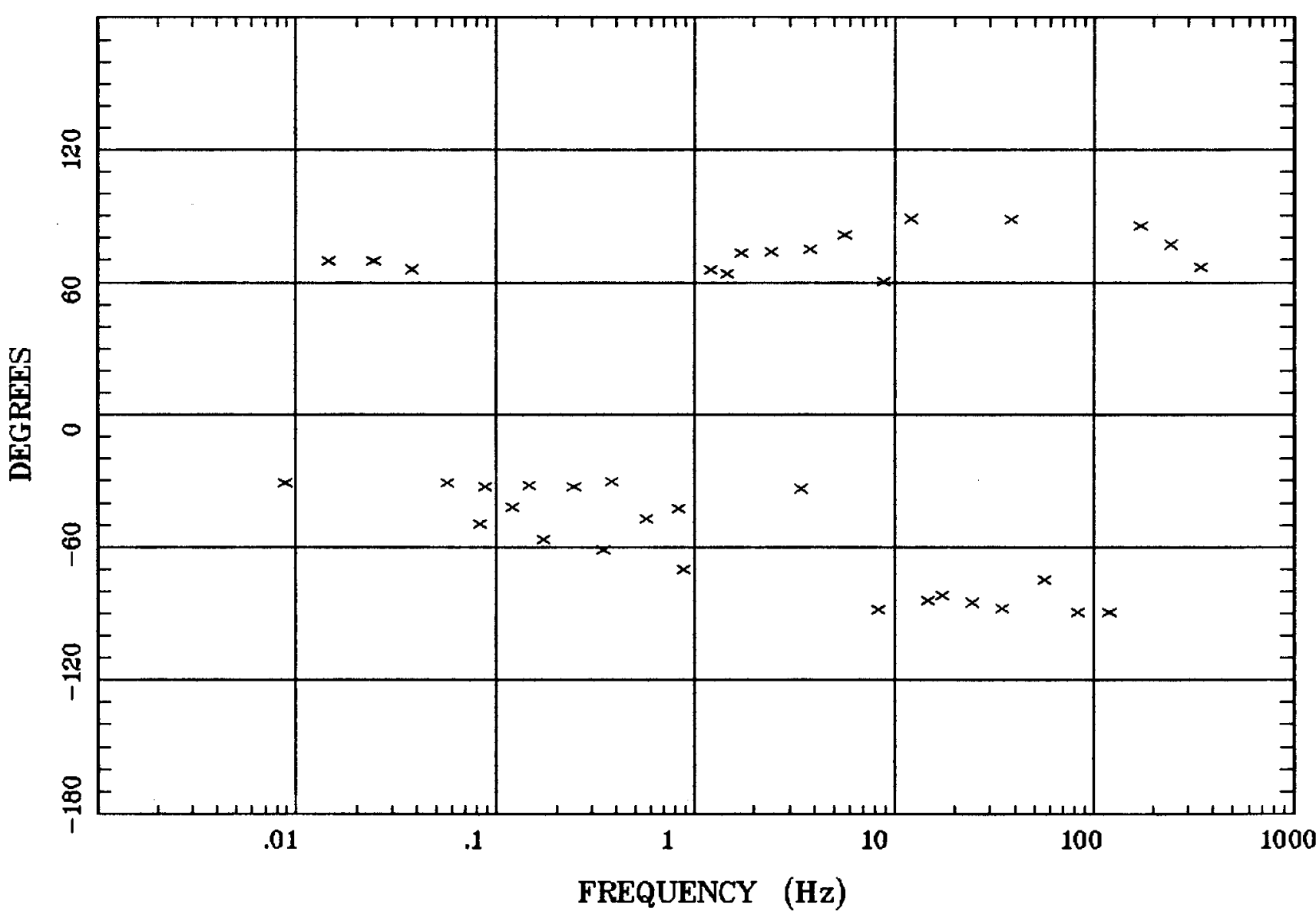

Client:

Remote: e-field local ref

Acquired: 17:0 Jul 09, 1997 Survey Co:
Rotation:

Filename: nna12,all

Channels: Ch1 Ch2 Ch3 Ch4 Ch5 Ch6 Ch7 Plotted: 08:51 Dec 08, 2000

< EMI - ElectroMagnetic Instruments > 
IMPEDANCE SKEW

battle mtn No. NV

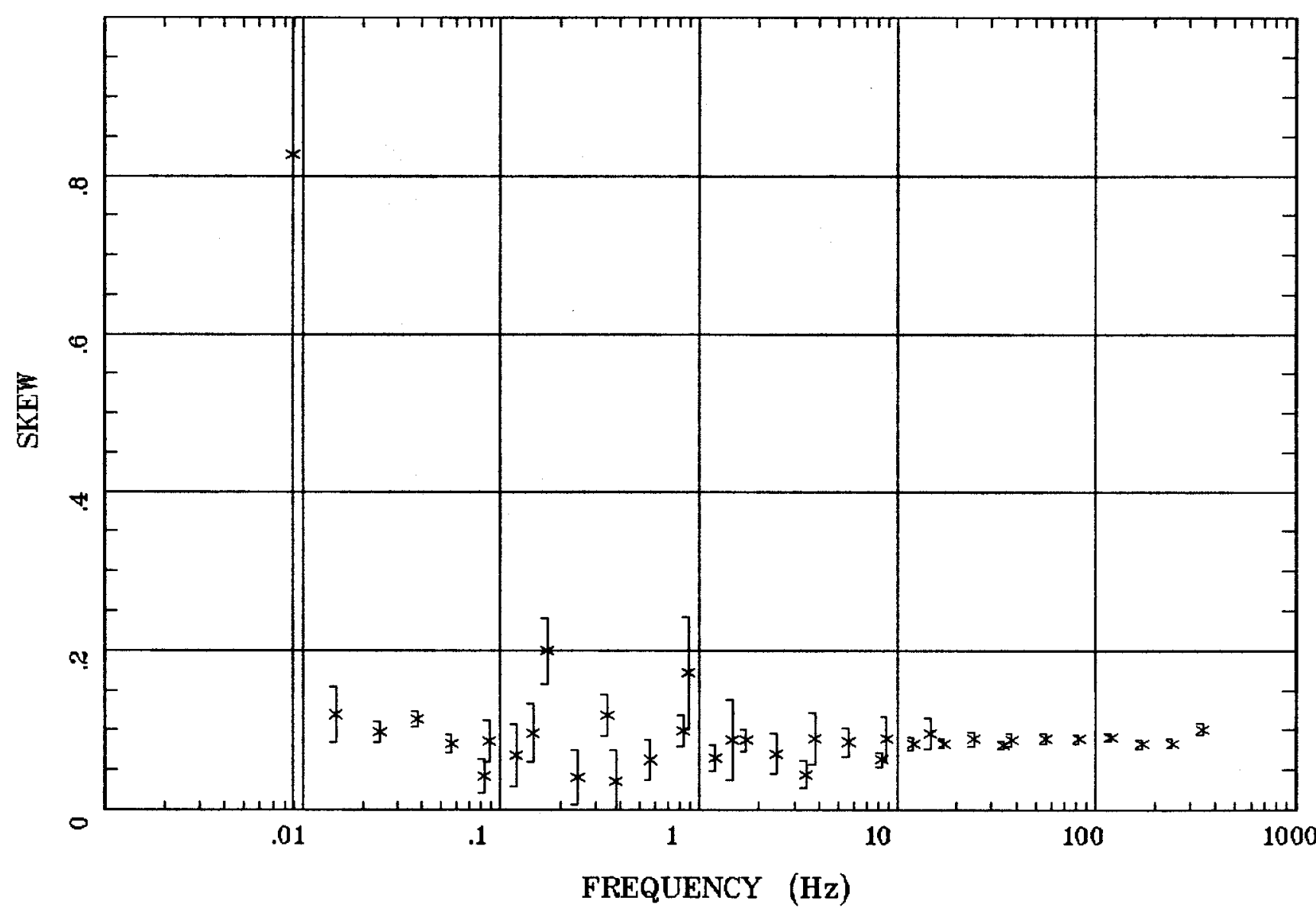

Client:

Remote: e-field local ref Acquired: 17:0 Jul 09, 1997 Survey Co:

Rotation:

Filename: nna12.all

Channels: Ch1 Ch2 Ch3 Ch4 Ch5 Ch6 Ch7 Plotted: 08:51 Dec 08, 2000

< EMI - ElectroMagnetic Instruments 
Station 12

E MULT Coh.

battle mtn No. NV

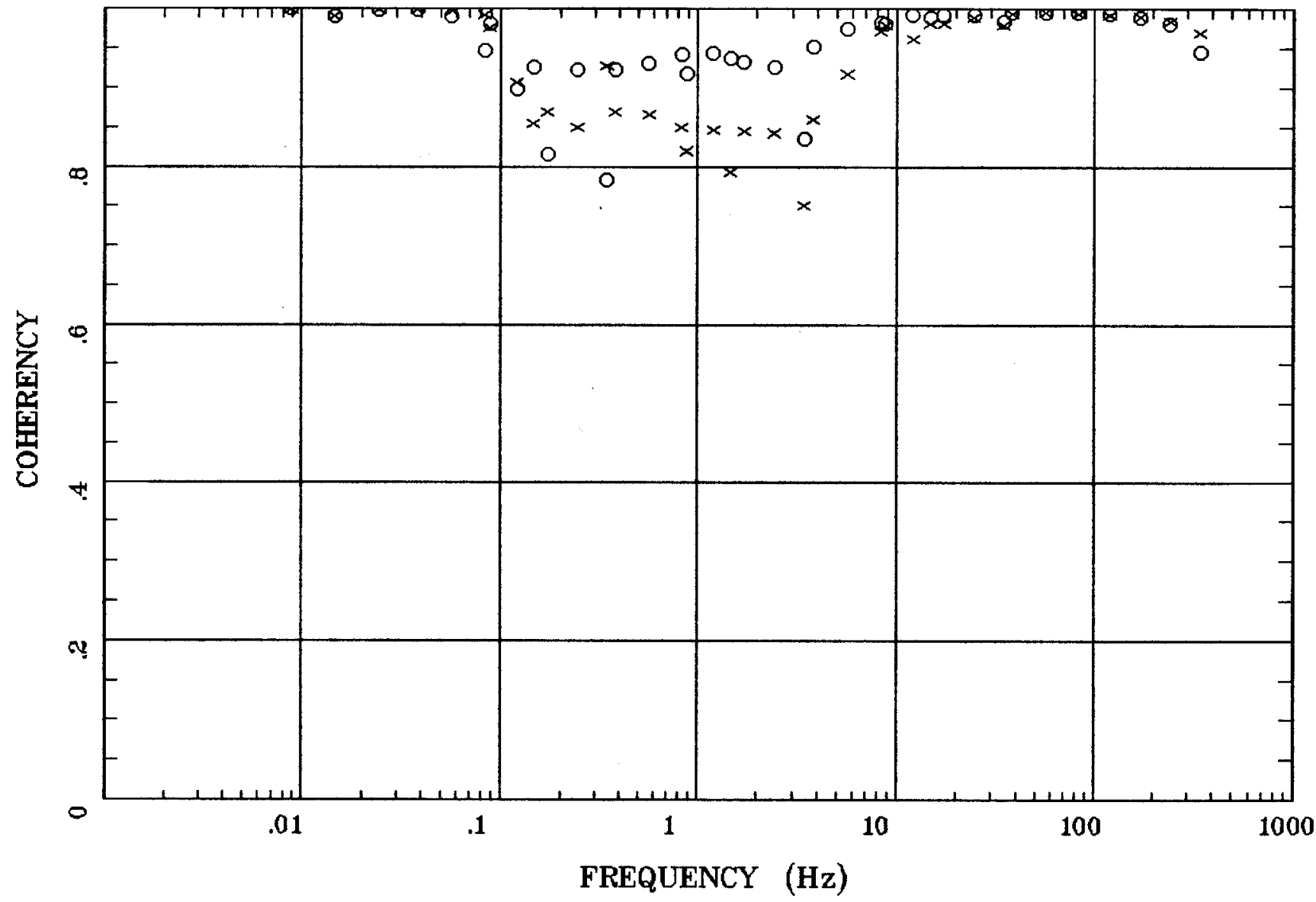

Client:

Remote: e-field local ref Acquired: 17:0 Jul 09, 1997 Survey Co:
Rotation:

Filename: nna12.all

Channels: Ch1 Ch2 Ch3 Ch4 Ch5 Ch6 Ch7

Plotted: 08:51 Dec 08, 2000

< EMI - ElectroMagnetic Instruments 
TIPPER MAGNITUDE

battle mtn No. NV

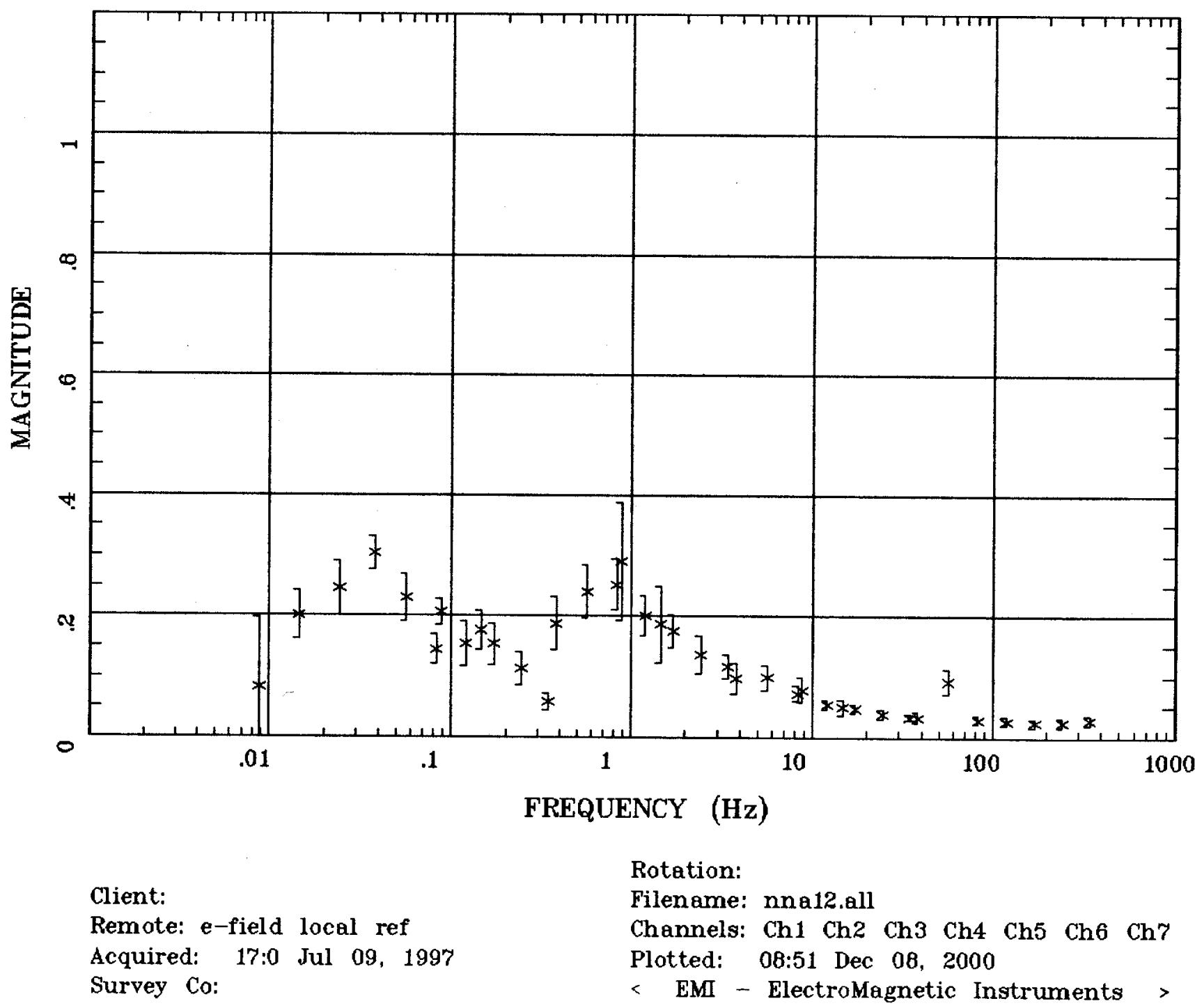


TIPPER STRIKE

battle mtn No. NV

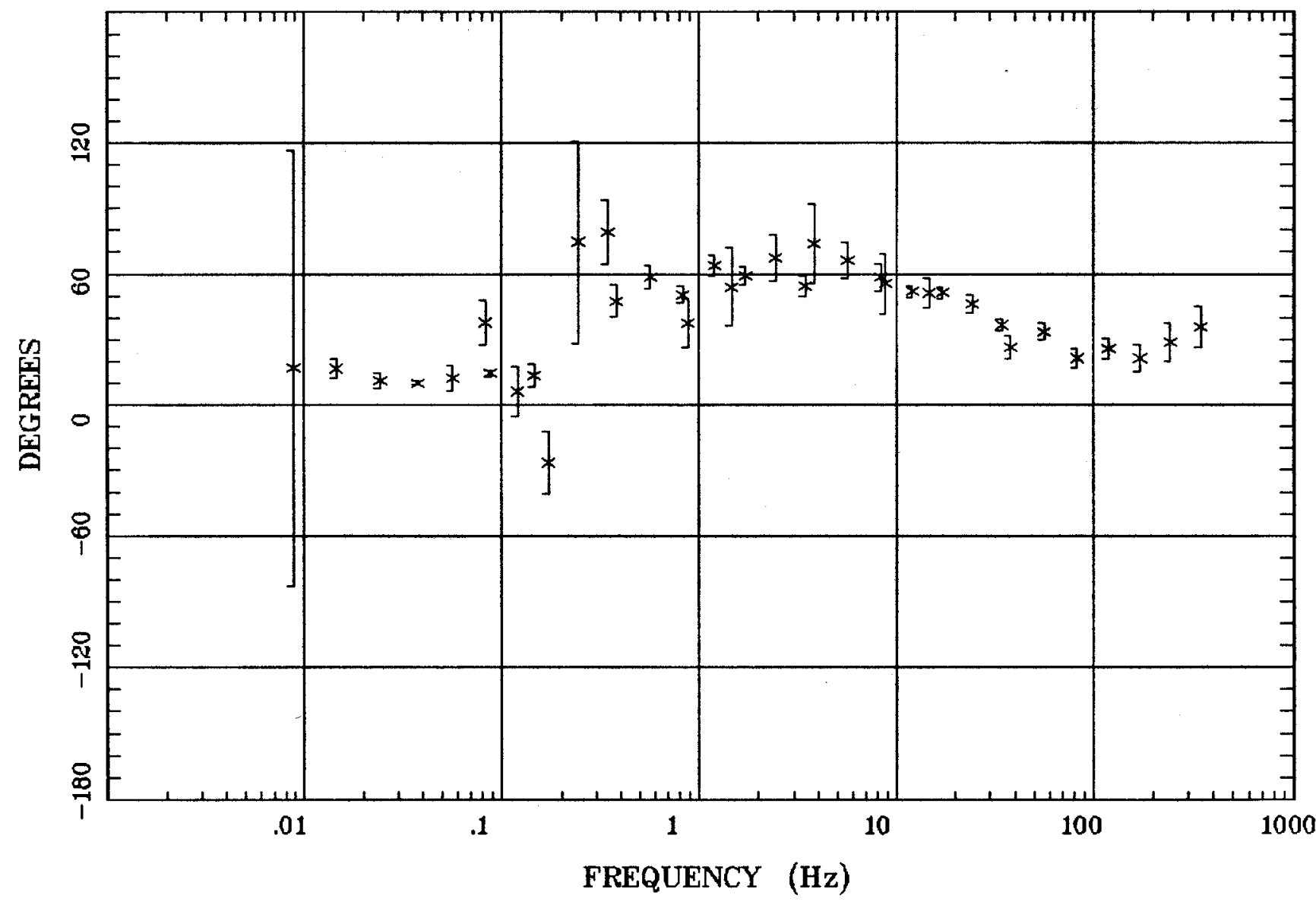

Client:

Remote: e-field local ref Acquired: 17:0 Jul 09, 1997 Survey Co:
Rotation:

Filename: nna12,all

Channels: Ch1 Ch2 Ch3 Ch4 Ch5 Ch6 Ch7

Plotted: 08:51 Dec 08, 2000

< EMI - ElectroMagnetic Instruments 
HzHx.x Coh HzHy.o

battle mtn No. NV

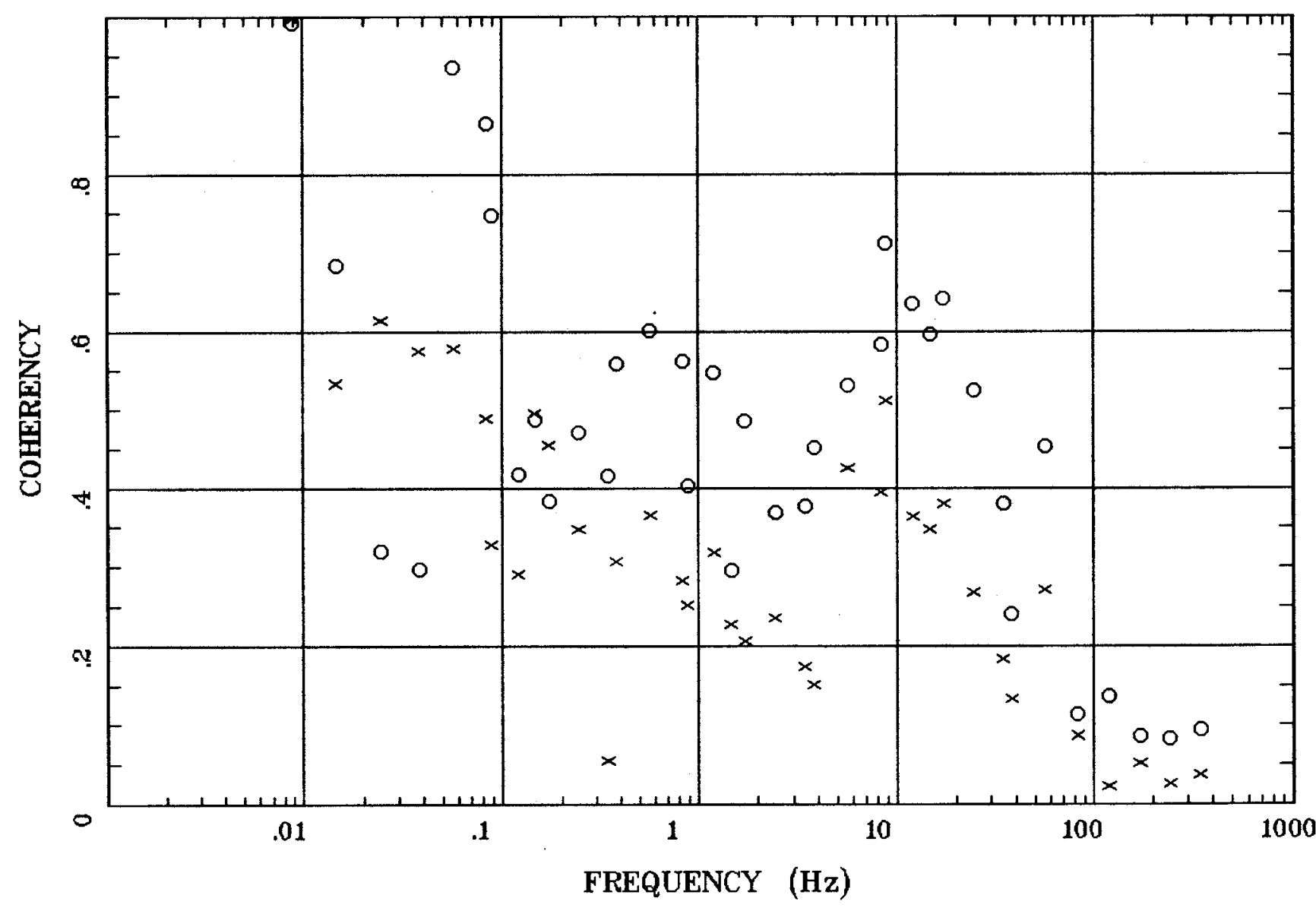

Client:

Remote: e-field local ref Acquired: 17:0 Jul 09, 1997 Survey Co:
Rotation:

Filename: nna12.all

Channels: Ch1 Ch2 Ch3 Ch4 Ch5 Ch6 Ch7 Plotted: 08:51 Dec 08, 2000

$<$ EMI - ElectroMagnetic Instruments 


\section{Station 13}

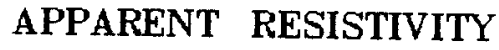

Battle Mtn.

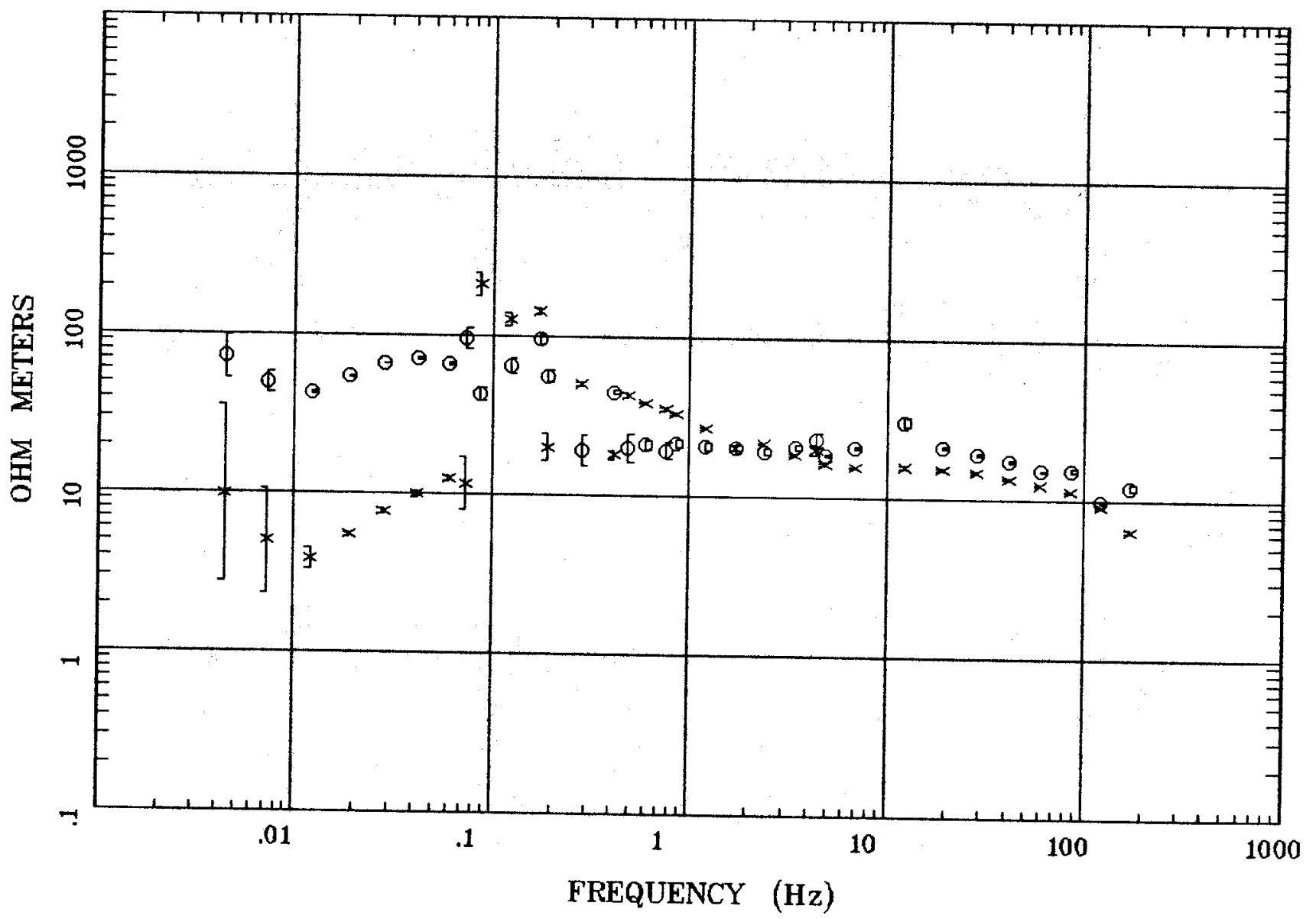

Client:

Remote: E local ref.

Acquired: 09:4 Jul 16, 1997

Survey Co:

\section{Rotation:}

Filename: nn13a.all

Channels: Ch1 Ch2 Ch3 Ch4 Ch5 Ch6 Ch7 Plotted: 08:26 Dec 08, 2000

< EMI - ElectroMagnetic Instruments 
IMPEDANCE PHASE

Battle Mtn.

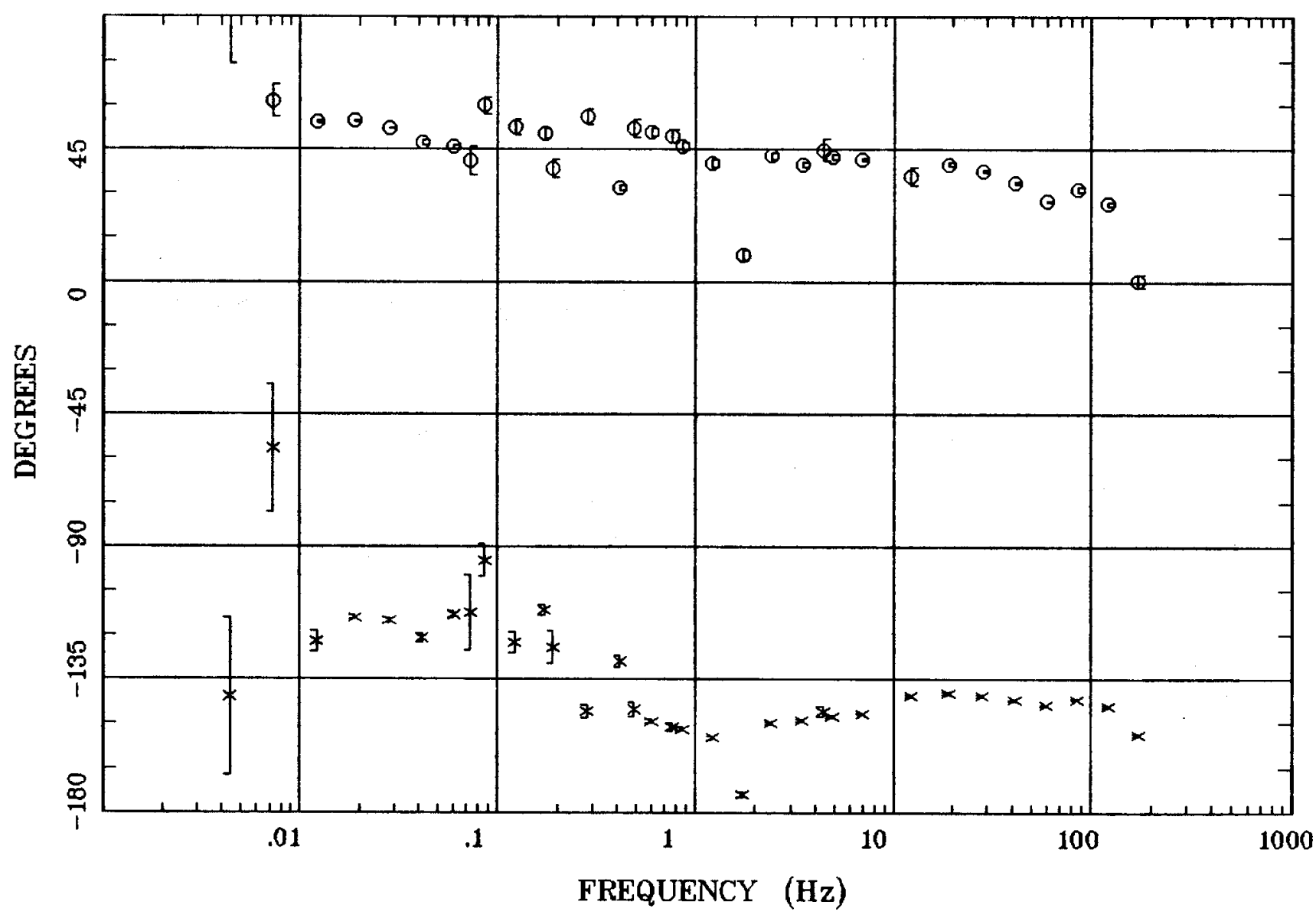

Client:

Remote: E local ref.

Acquired: 09:4 Jul 16, 1997

Survey Co:
Rotation:

Filename: nn13a.all

Channels: Ch1 Ch2 Ch3 Ch4 Ch5 Ch6 Ch7

Plotted: 08:26 Dec 08, 2000

< EMI - ElectroMagnetic Instruments 
Station 13

ROTATION ANGLE

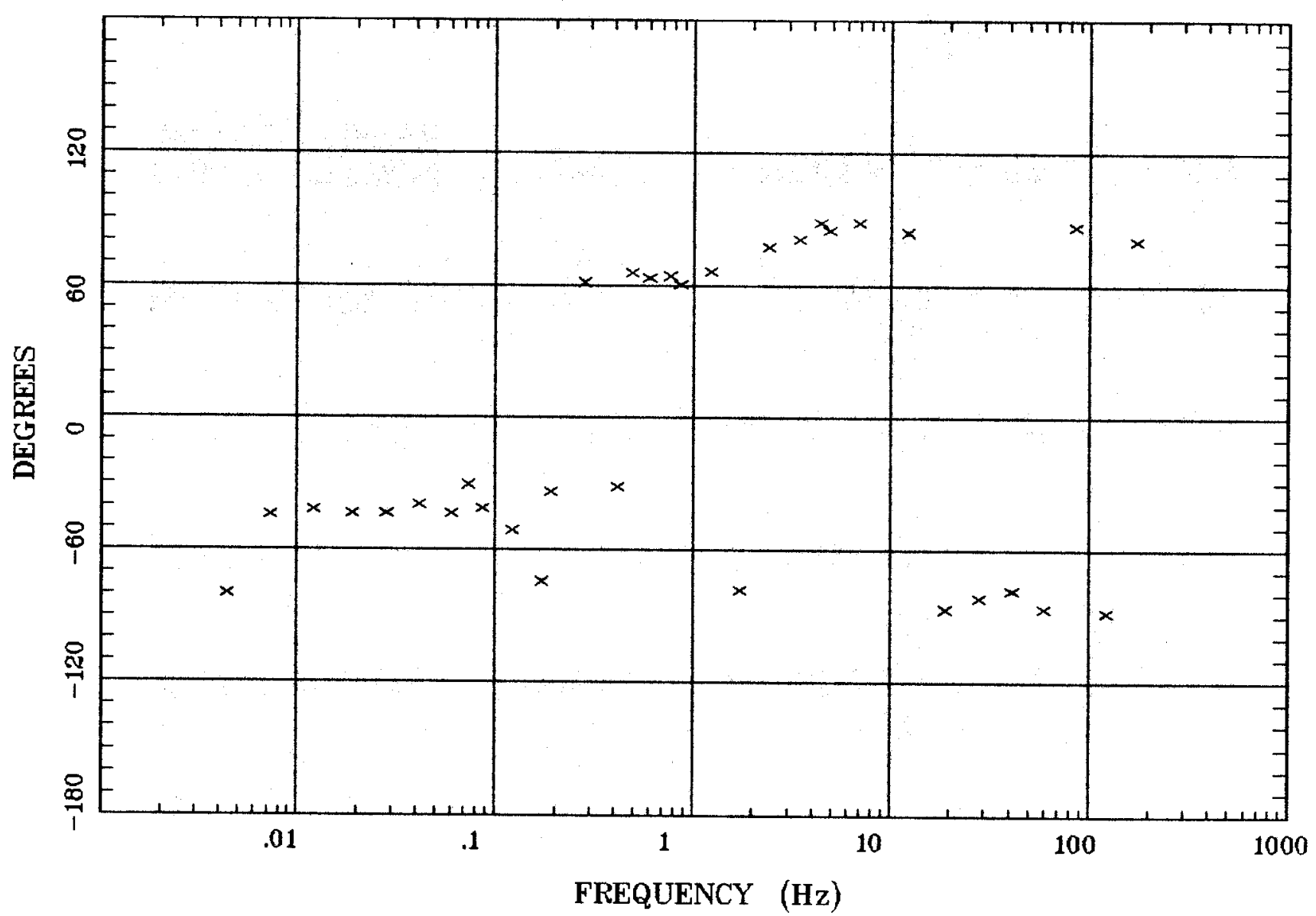

Client:

Remote: E local ref.

Acquired: 09:4 Jul 16, 1997

Survey Co:
Rotation:

Filename: nn13a.all

Channels: Ch1 Ch2 Ch3 Ch4 Ch5 Ch6 Ch7

Plotted: 08:26 Dec 08, 2000

< EMI - ElectroMagnetic Instruments > 
IMPEDANCE SKEW

Battle Mtn.

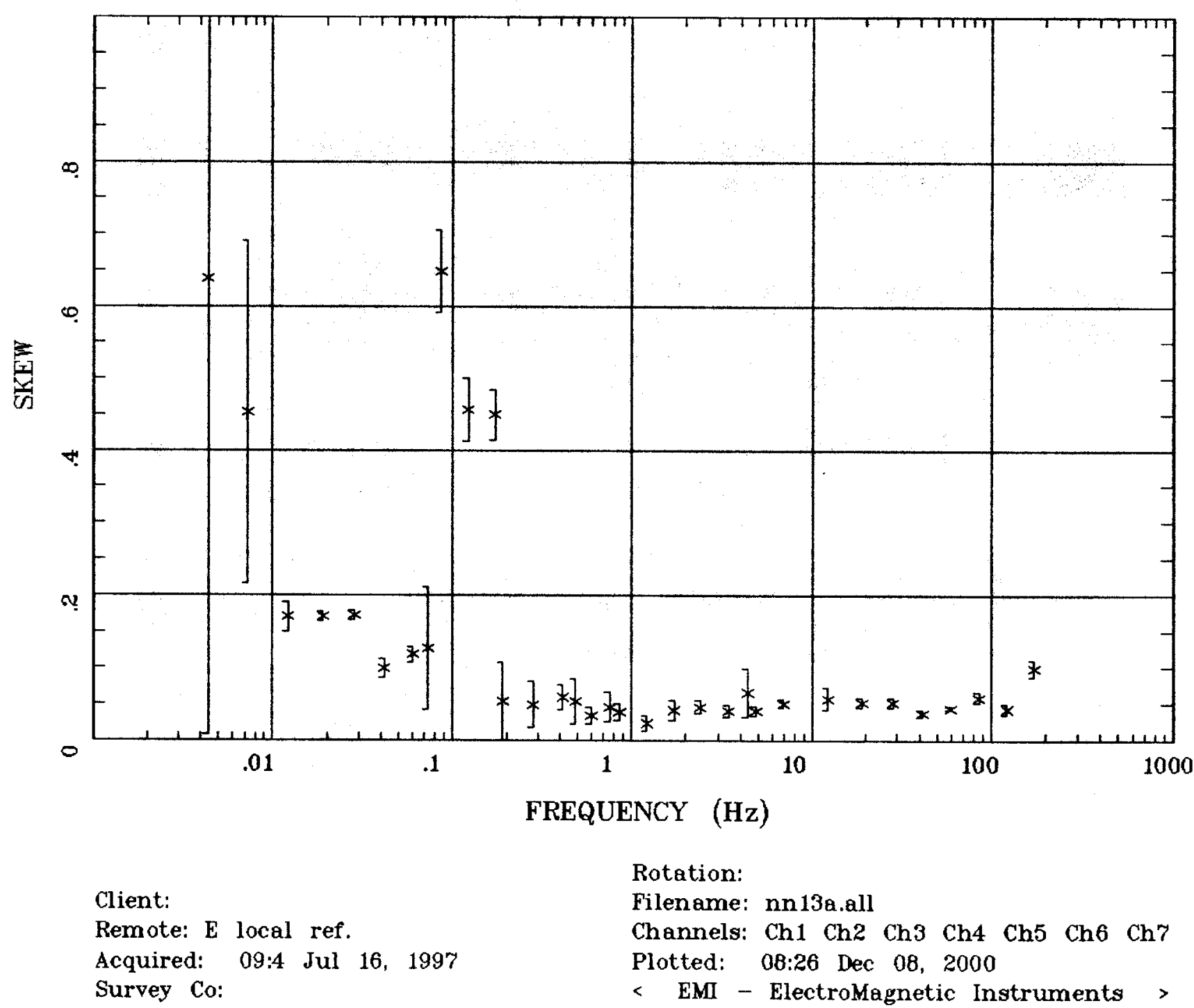


Station 13

E MULT Coh.

Battle Mtn.

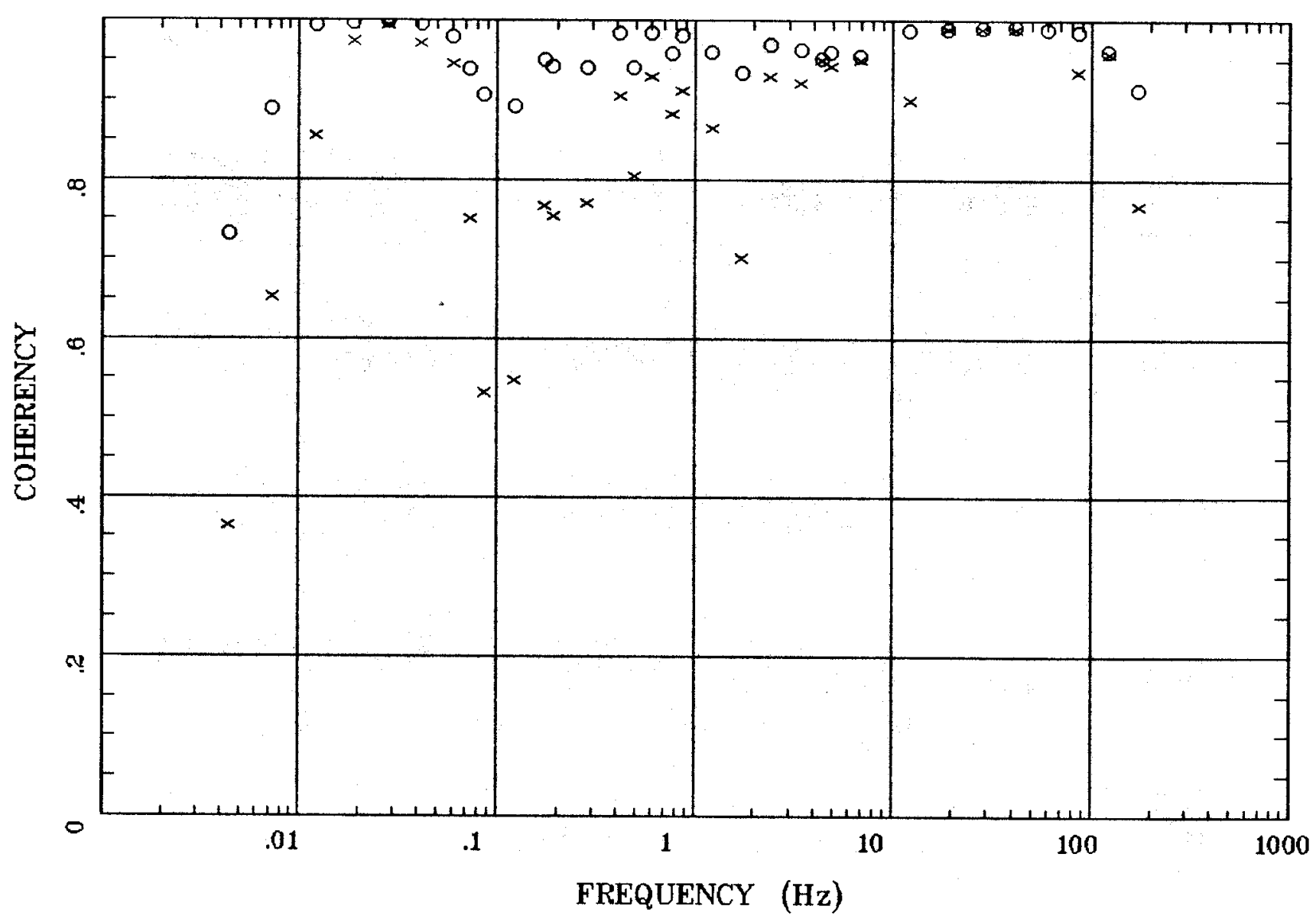

Client:

Remote: E local ref.

Acquired: 09:4 Jul 16, 1997

Survey Co:
Rotation:

Filename: nn13a.all

Channels: Ch1 Ch2 Ch3 Ch4 Ch5 Ch6 Ch7

Plotted: 08:26 Dec 08, 2000

< EMI - ElectroMagnetic Instruments > 
Battle Mtn.

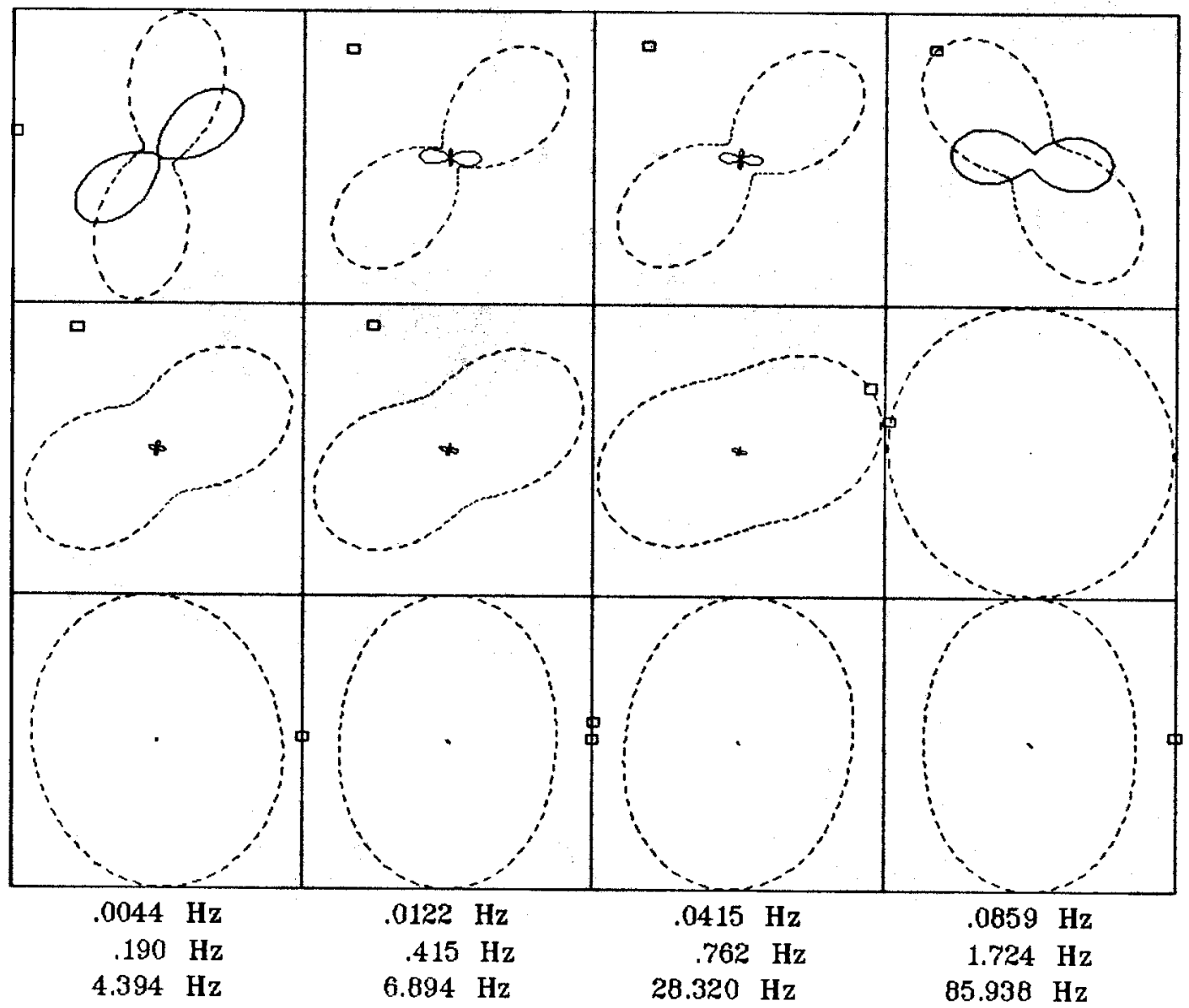

Client:

Remote: E local ref.

Acquired: 09:4 Jul 16, 1997 Survey Co:
Rotation:

Filename: nn13a.all

Channels: Ch1 Ch2 Ch3 Ch4 Ch5 Ch6 Ch7

Plotted: 08:26 Dec 08, 2000

< EMI - ElectroMagnetic Instruments 
Battle Mtn.

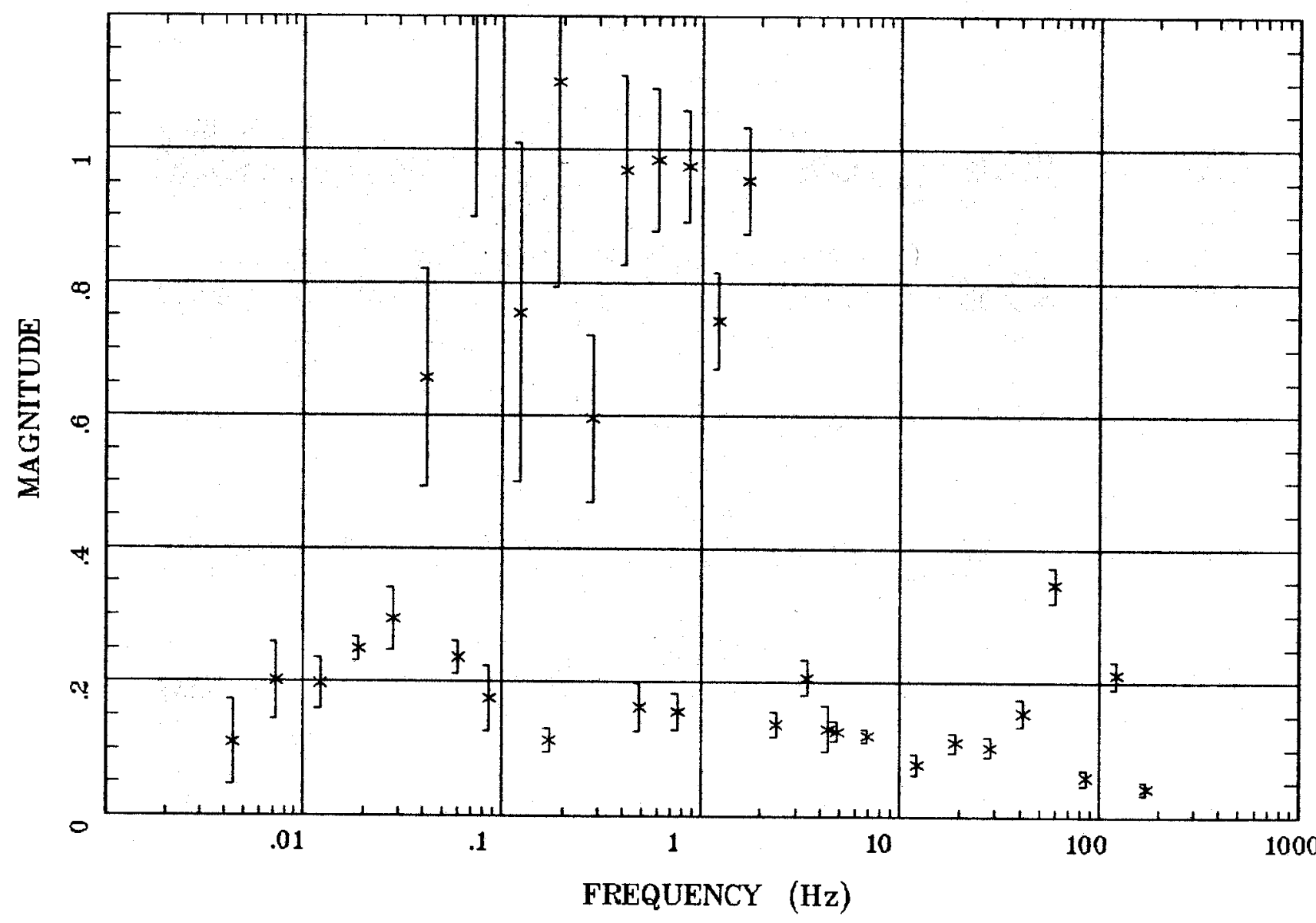

Client:

Remote: E local ref.

Acquired: 09:4 Jul 16, 1997 Survey Co:
Rotation:

Filename: nn 13a.all

Channels: Ch1 Ch2 Ch3 Ch4 Ch5 Ch6 Ch7 Plotted: 08:26 Dec 08, 2000

$<$ EMI - ElectroMagnetic Instruments 


\section{Station 13}

\section{TIPPER STRIKE}

Battle Mtn.

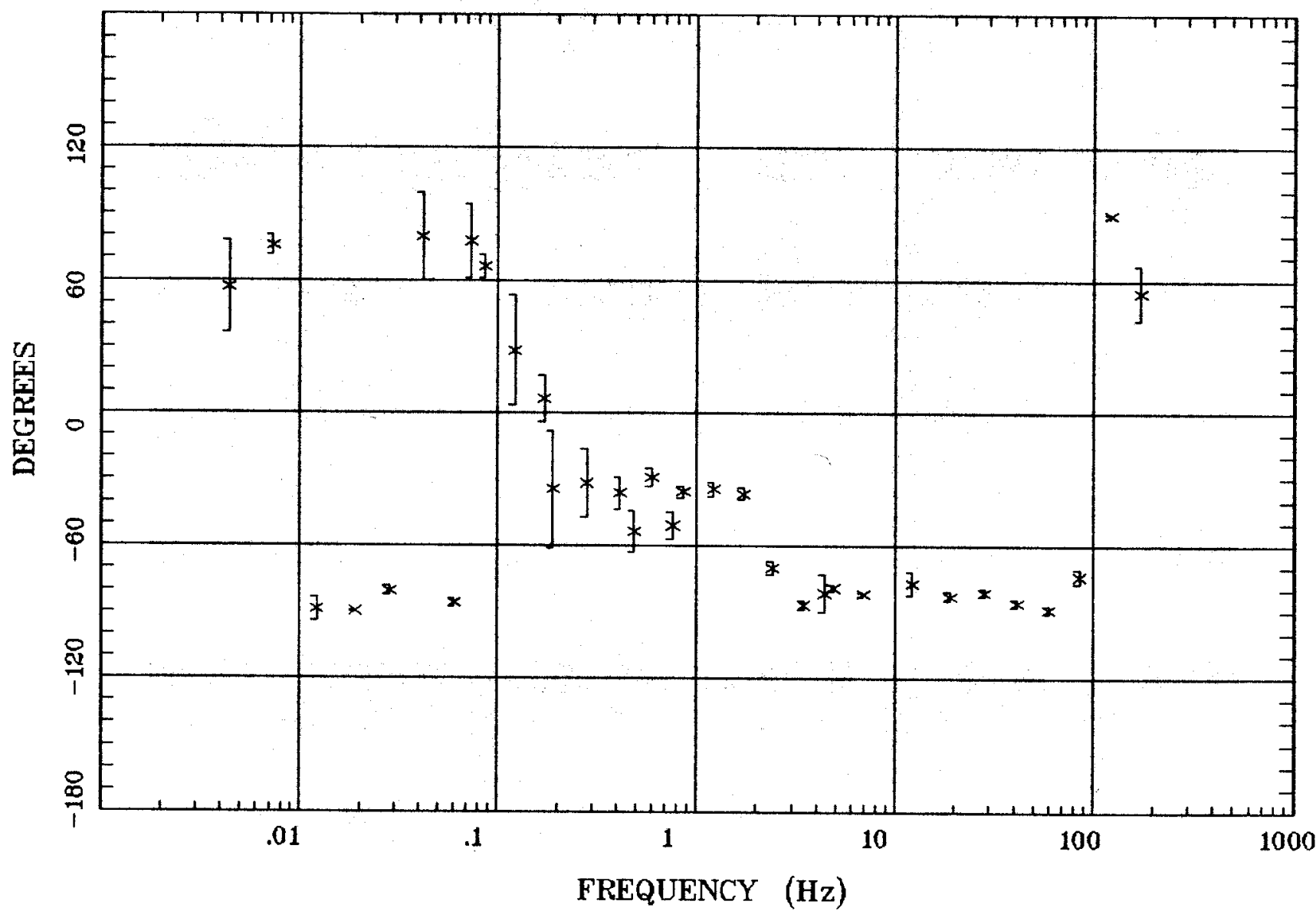

Client:

Remote: E local ref.

Acquired: 09:4 Jul 16, 1997

Survey Co:
Rotation:

Filename: nn13a.all

Channels: Ch1 Ch2 Ch3 Ch4 Ch5 Ch6 Ch7

Plotted: 08:26 Dec 08, 2000

< EMI - ElectroMagnetic Instruments 


\section{HzHx.x Coh HzHy.o}

Battle Mtn.

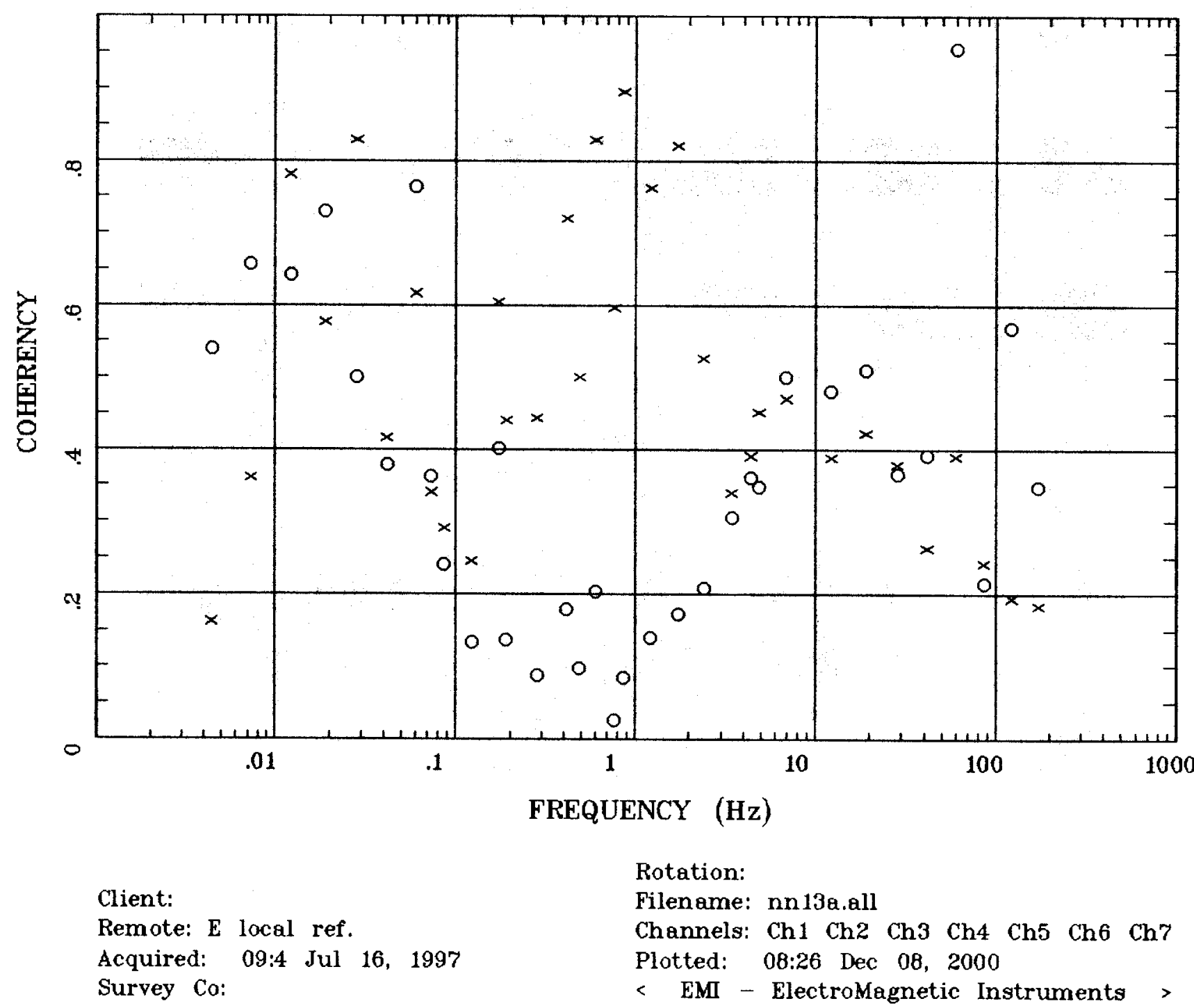




\section{Station 14}

APPARENT RESISTIVITY

Battle Mtn.

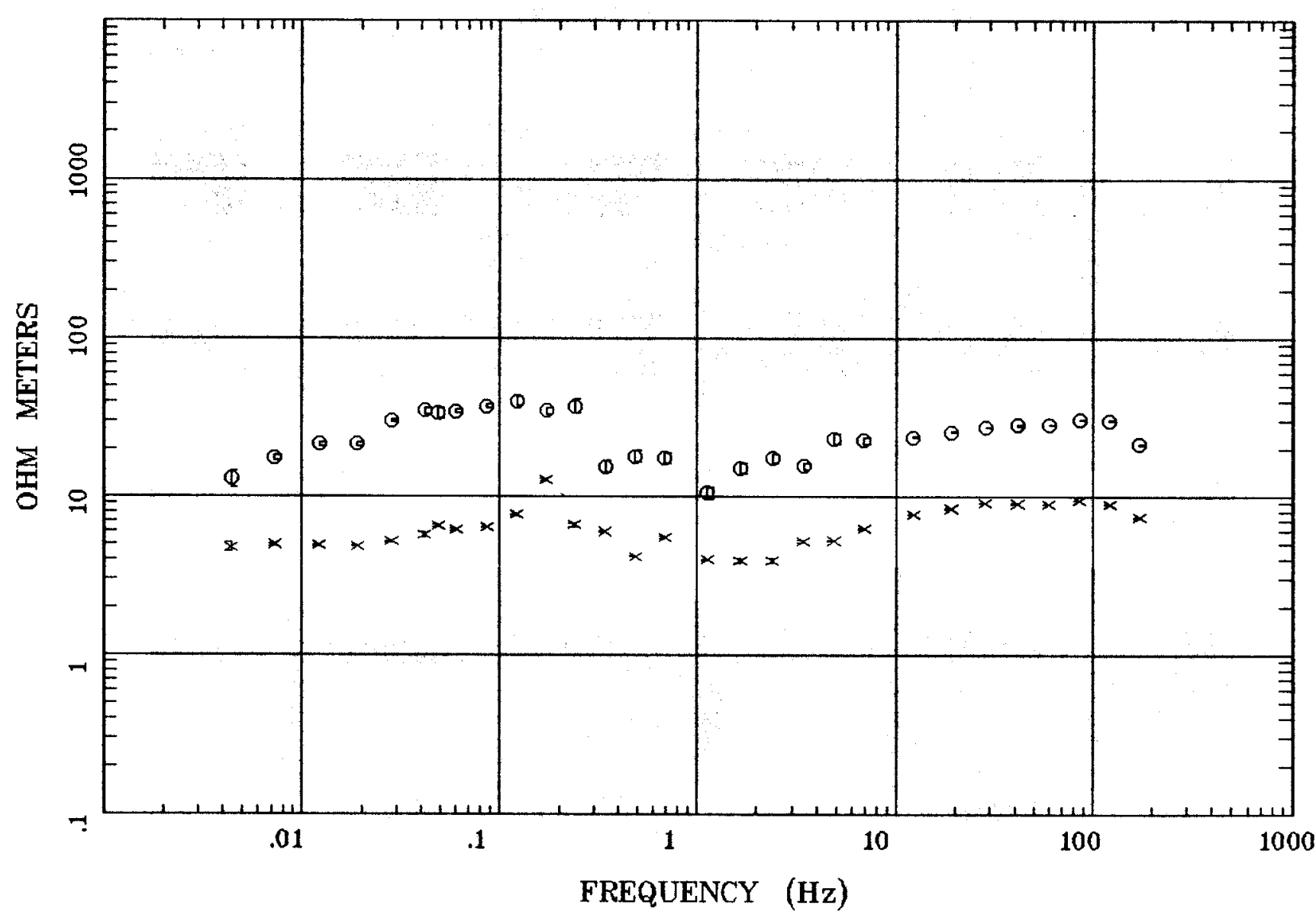

Client:

Remote: E local ref.

Acquired: 10:0 Jul 17, 1997 Survey Co:

\section{Rotation:}

Filename: nn14.all

Channels: Ch1 Ch2 Ch3 Ch4 Ch5 Ch6 Ch7

Plotted: 08:27 Dec 08, 2000

$<$ EMI - ElectroMagnetic Instruments > 
Station 14

IMPEDANCE PHASE

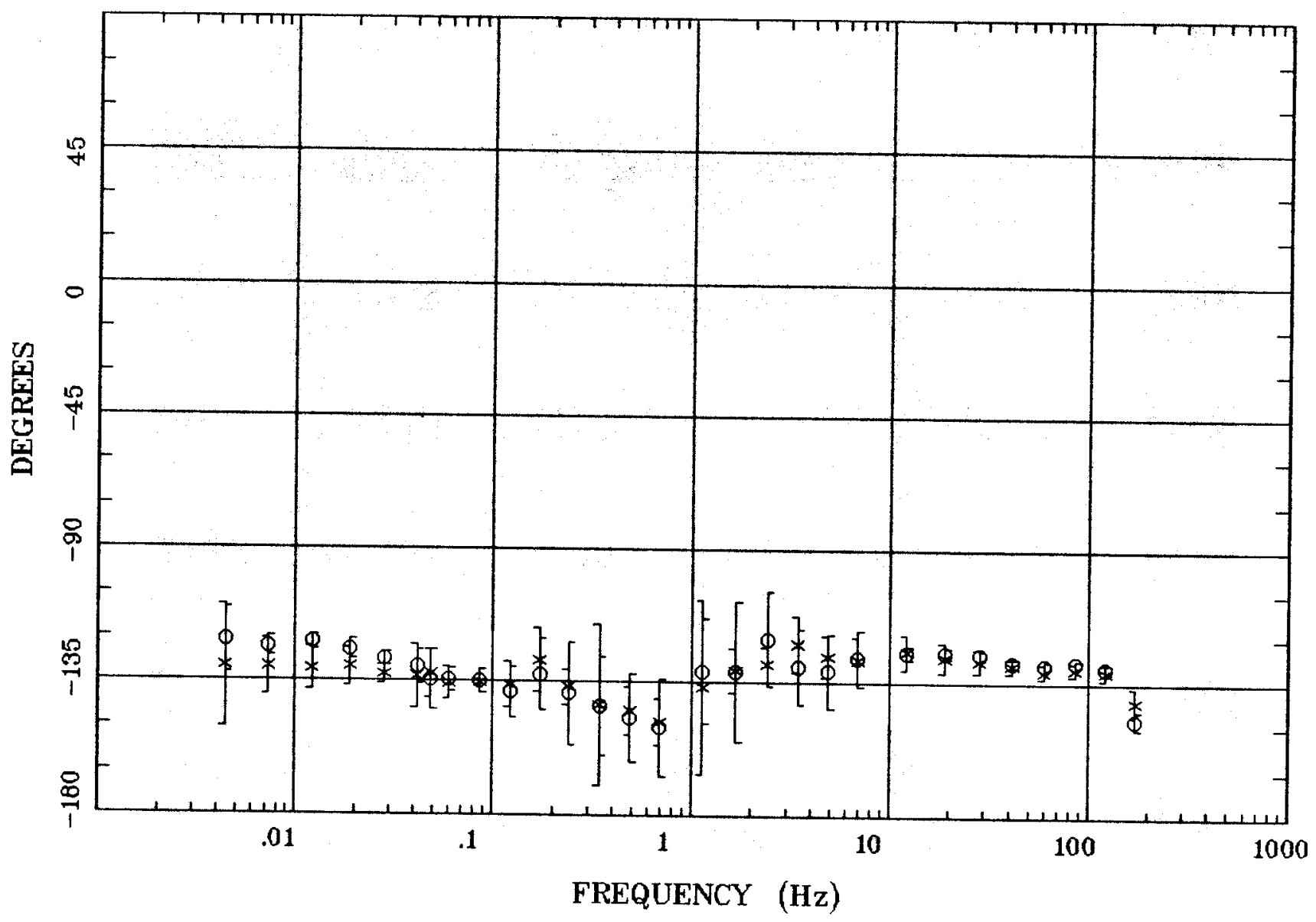

Client:

Remote: E local ref.

Acquired: 10:0 Jul 17, 1997 Survey Co:
Rotation:

Filename: nn14.all

Channels: Ch1 Ch2 Ch3 Ch4 Ch5 Ch6 Ch?

Plotted: 08:27 Dec 08, 2000

< EMI - ElectroMagnetic Instruments 


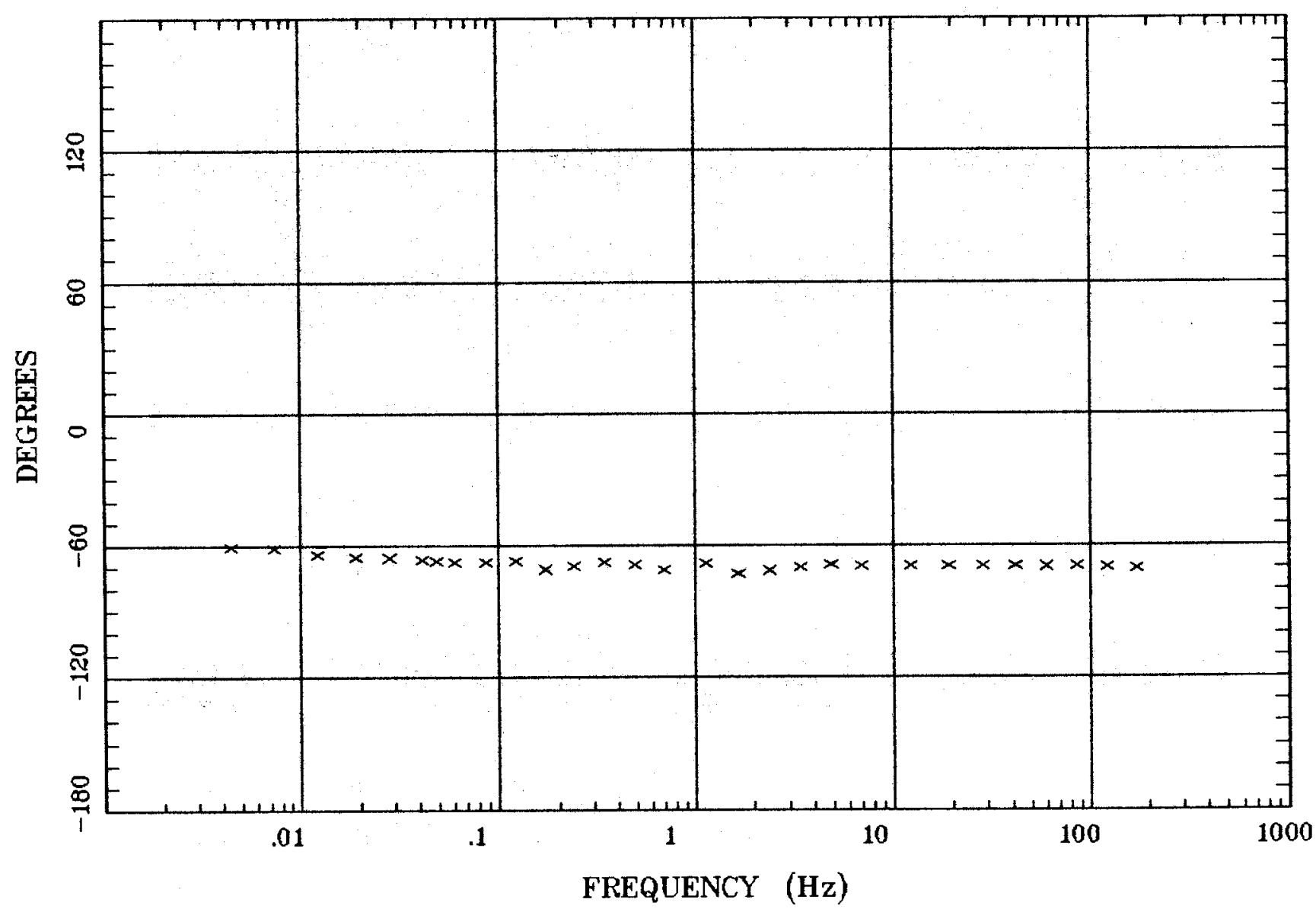

Client:

Remote: E local ref.

Acquired: 10:0 Jul 17, 1997 Survey Co:
Rotation:

Filename: nn14.all

Channels: Ch1 Ch2 Ch3 Ch4 Ch5 Ch6 Ch7

Plotted: 08:27 Dec 08, 2000

< EMI - ElectroMagnetic Instruments > 
IMPEDANCE SKEW

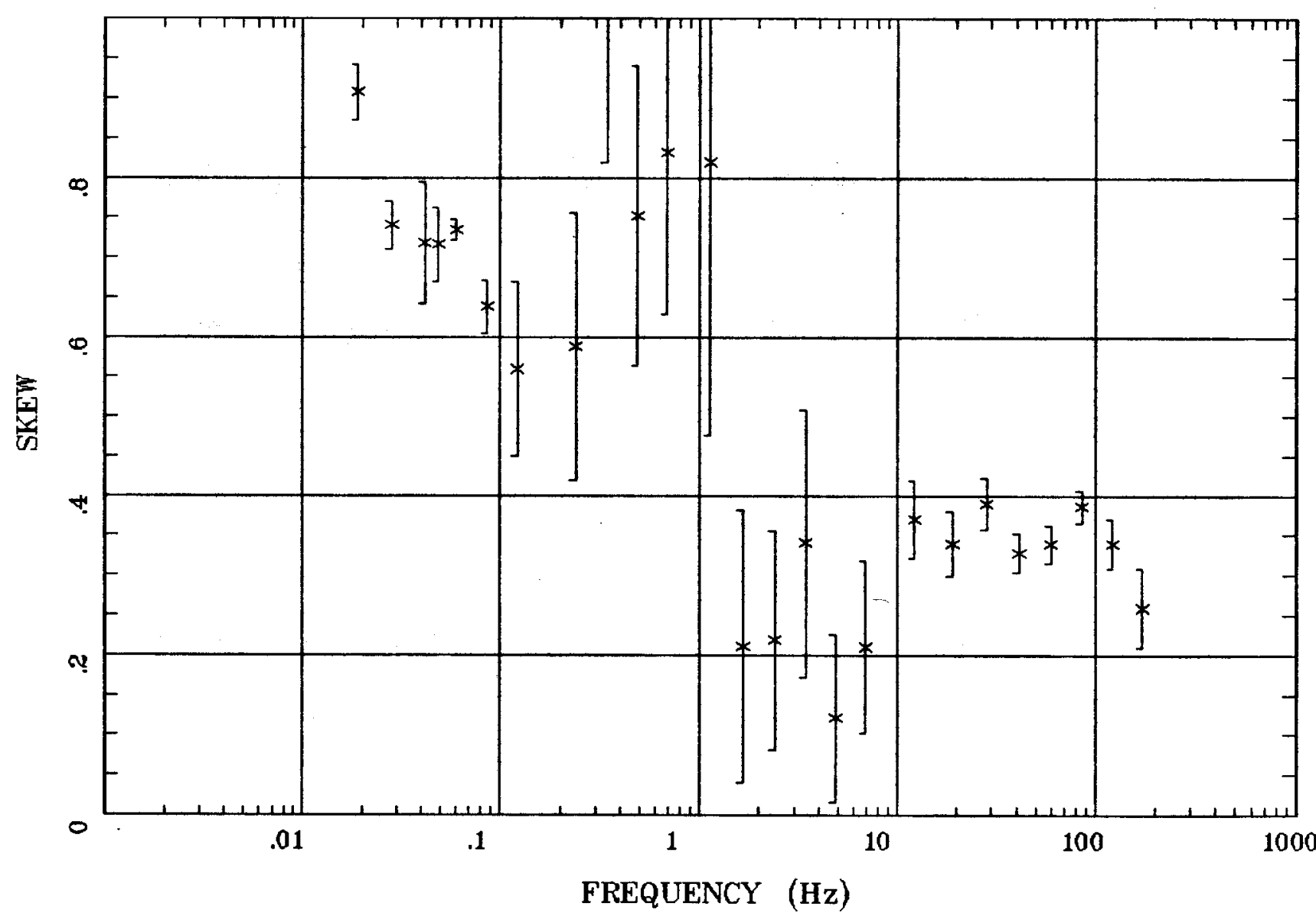

Client:

Remote: E local ref.

Acquired: 10:0 Jul 17, 1997 Survey Co:
Battle Mtn.

Rotation:

Filename: nn14.all

Channels: Ch1 Ch2 Ch3 Ch4 Ch5 Ch6 Ch7

< EMI - ElectroMagnetic Instruments
Plotted: 08:27 Dec 08, 2000 
E MULT Coh. Battle Mtn.

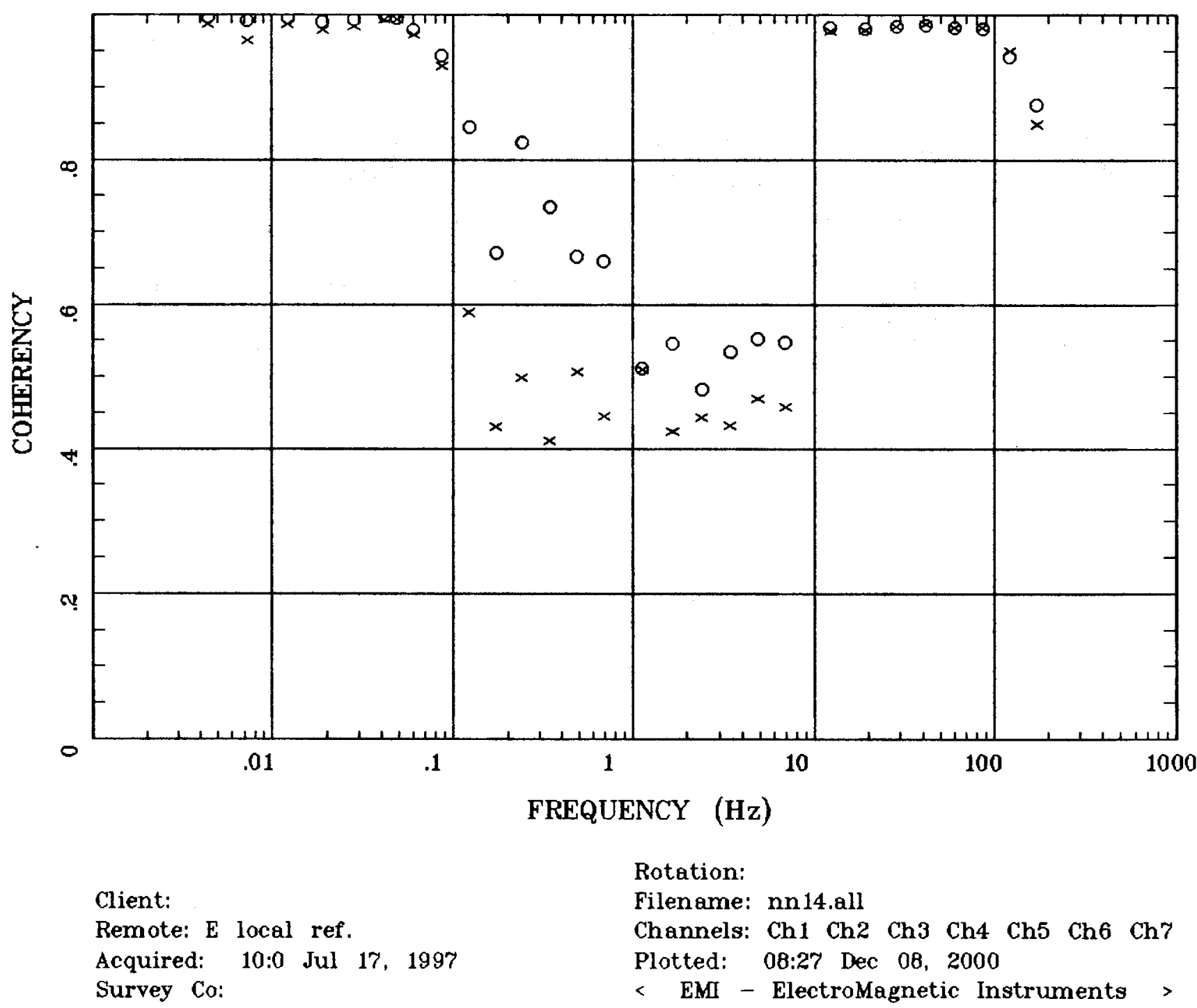


Battle Mtn.

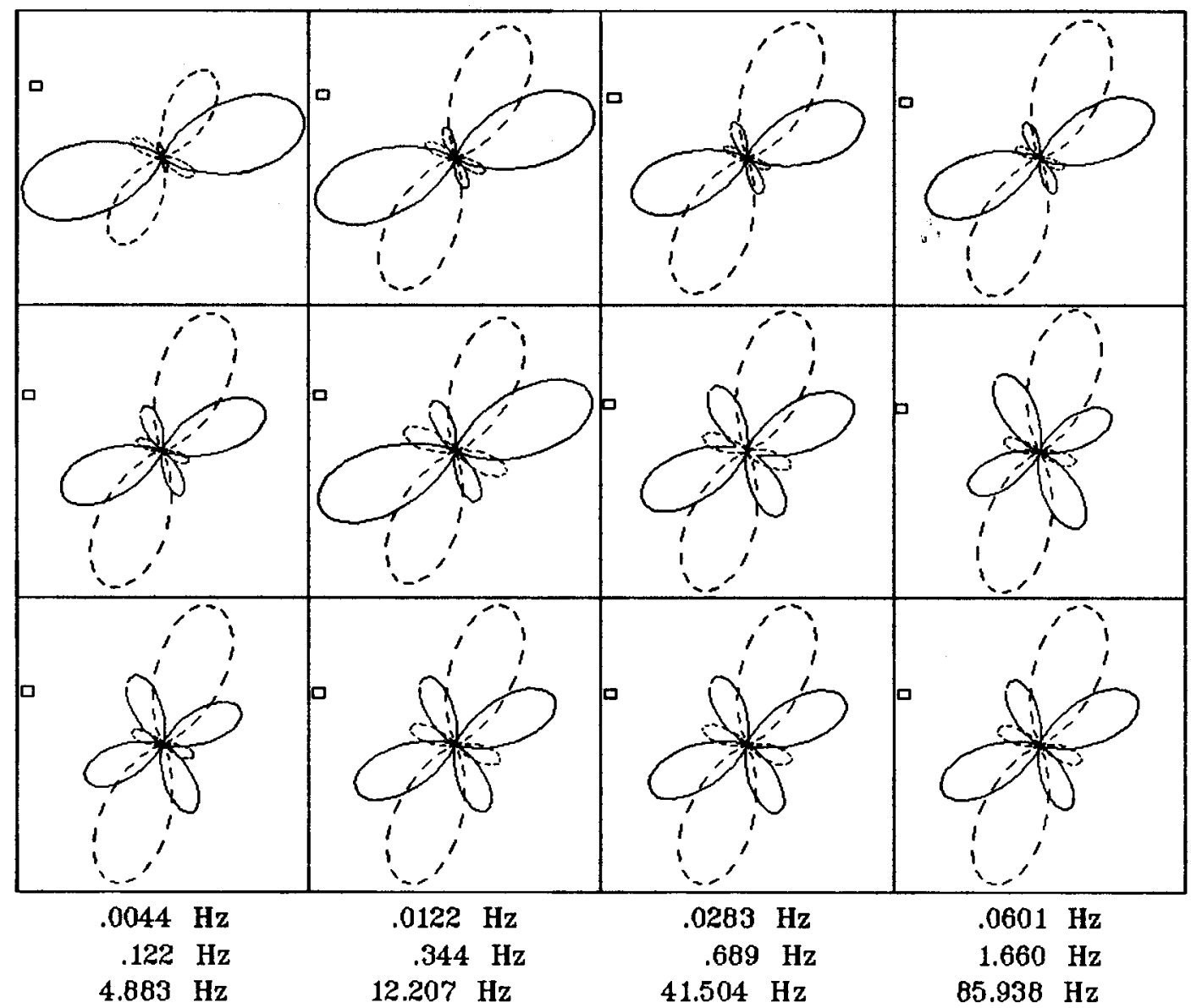

Client:

Remote: E local ref.

Acquired: 10:0 Jul 17, 1997

Survey Co:
Rotation:

Filename: nn14.all

Channels: Ch1 Ch2 Ch3 Ch4 Ch5 Ch6 Ch7

Plotted: 08:27 Dec 08, 2000

$\langle$ EMI - ElectroMagnetic Instruments > 
Battle Mtn.

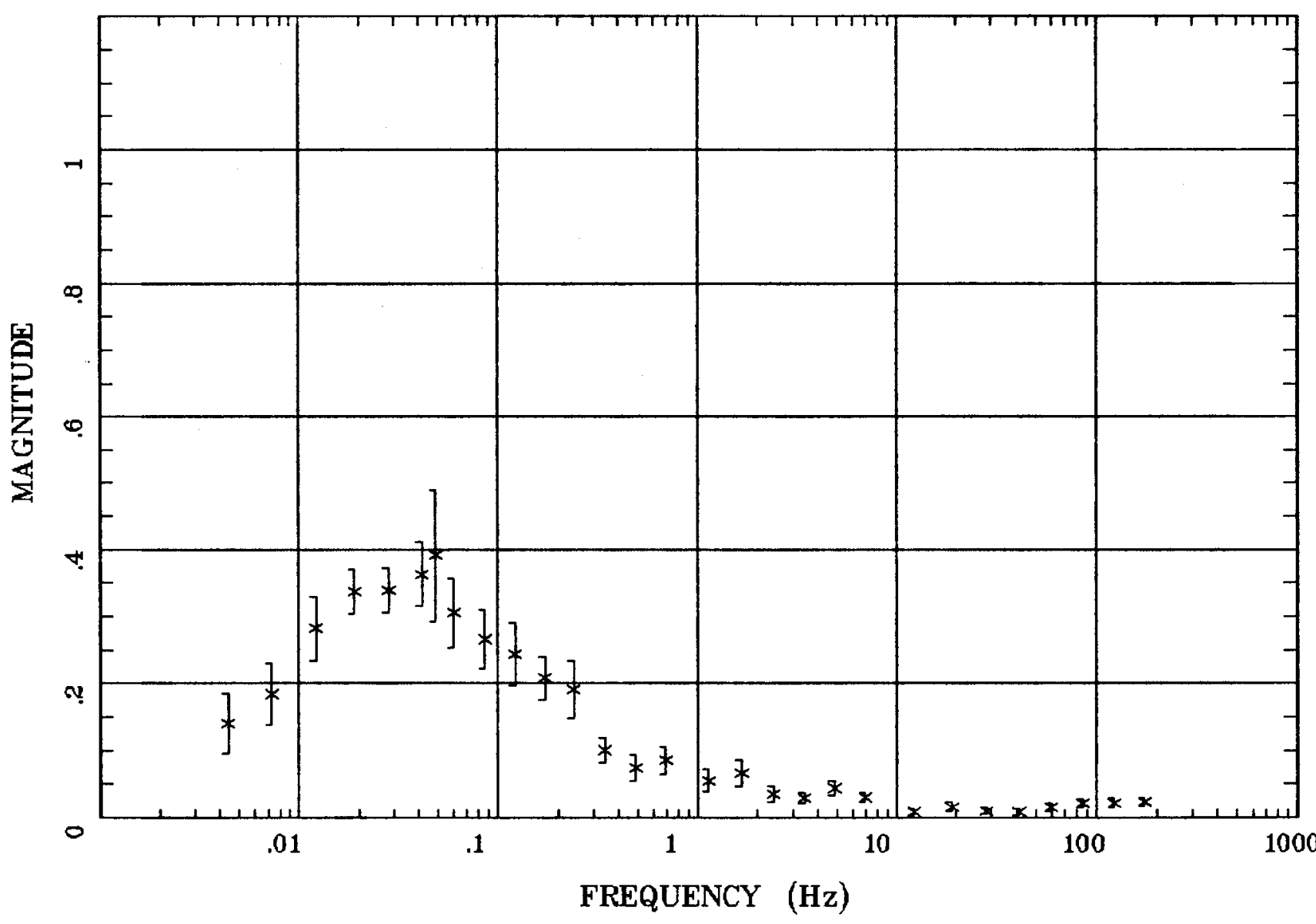

Client:

Remote: E local ref.

Acquired: 10:0 Jul 17, 1997

Survey Co:
Rotation:

Filename: nn14.all

Channels: Ch1 Ch2 Ch3 Ch4 Ch5 Ch6 Ch7

Plotted: 08:27 Dec 08, 2000

< EMI - ElectroMagnetic Instruments 
TIPPER STRIKE

Battle Mtn.

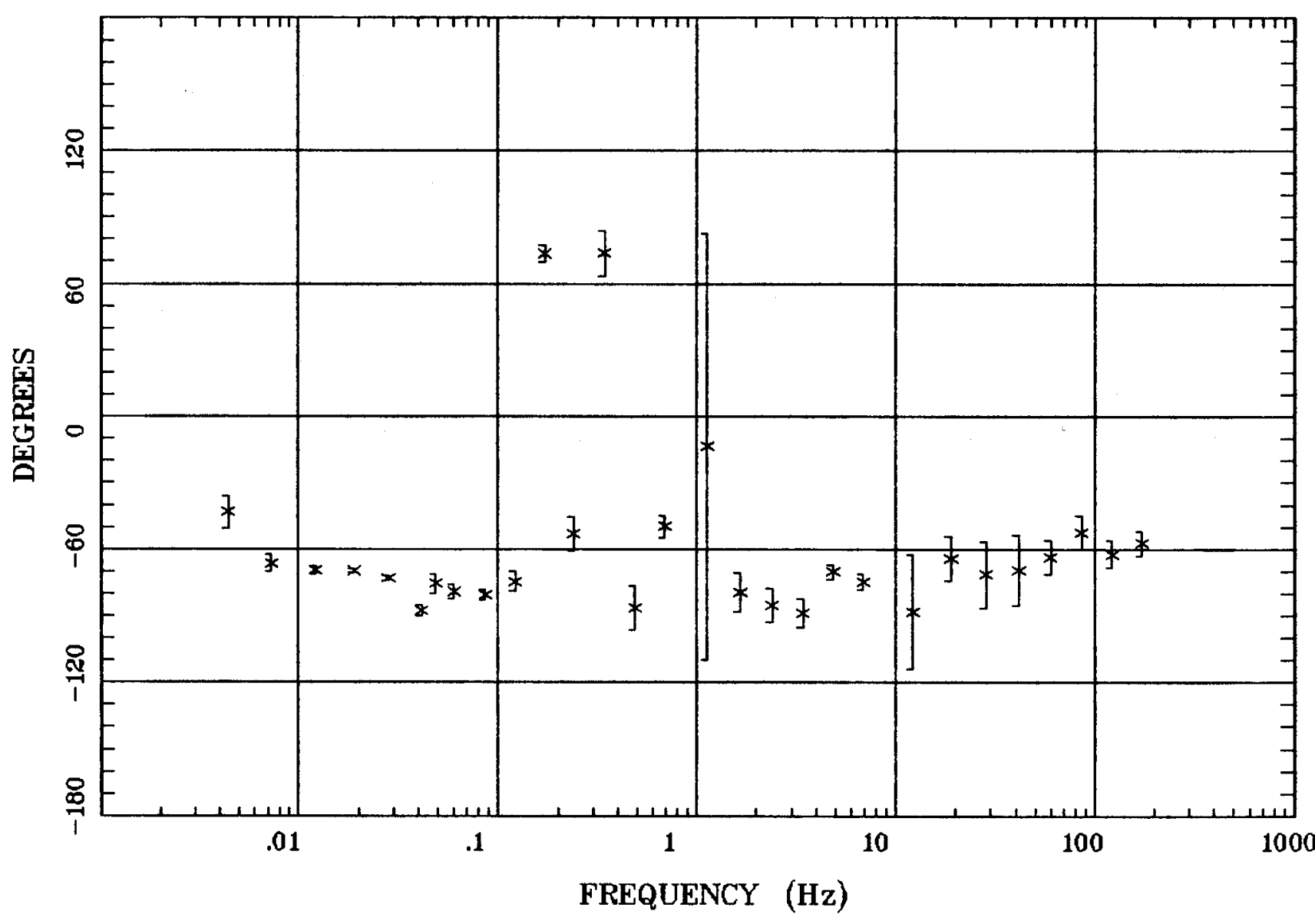

Client:

Remote: E local ref.

Acquired: 10:0 Jul 17, 1997 Survey Co:
Rotation:

Filename: nn14.all

Channels: Ch1 Ch2 Ch3 Ch4 Ch5 Ch6 Ch7

Plotted: 08:27 Dec 08, 2000

< EMI - ElectroMagnetic Instruments 
HzHx.x Coh HzHy.o

Station 14

Battle Mtn.

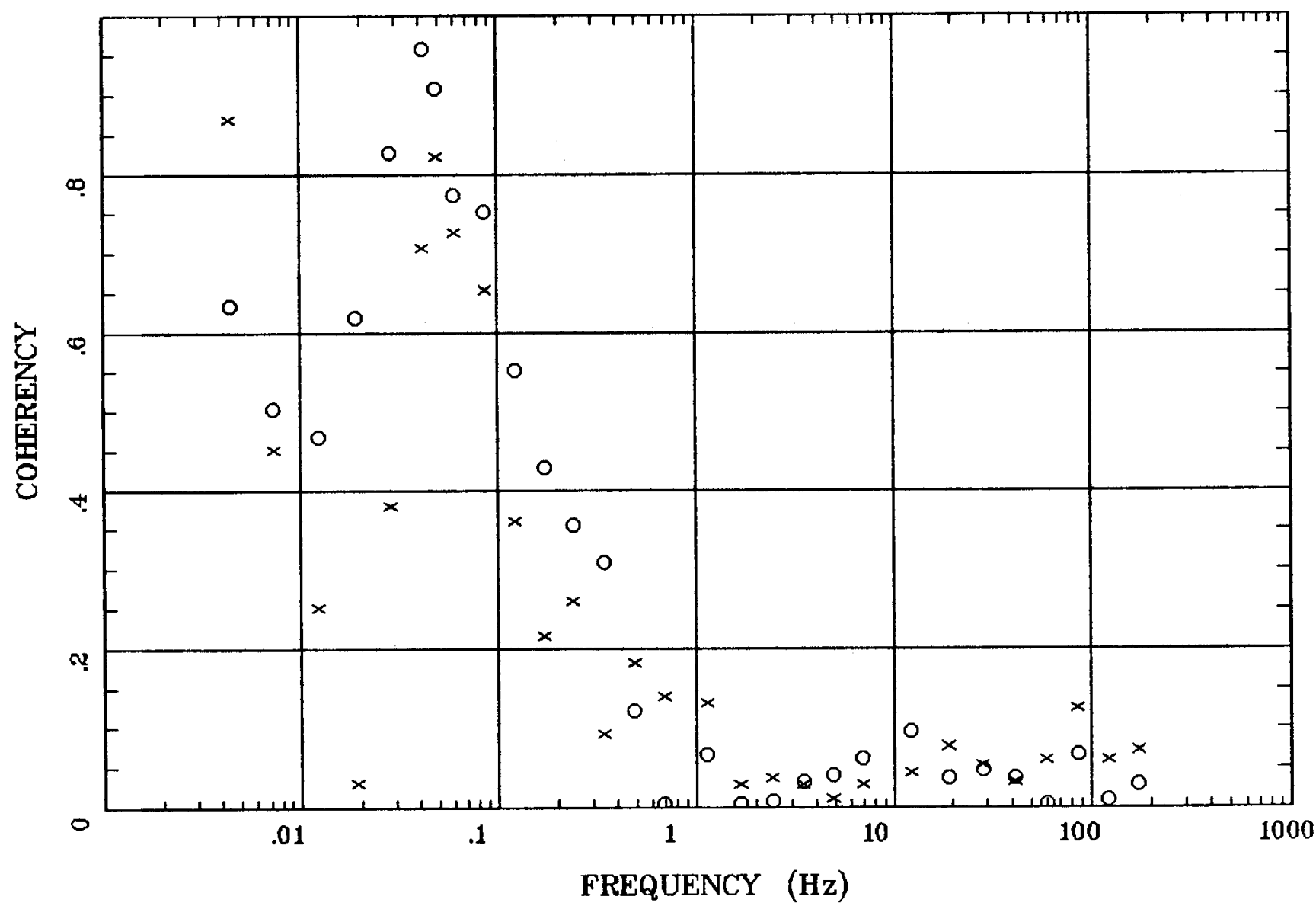

Client:

Remote: E local ref.

Acquired: 10:0 Jul 17, 1997 Survey Co:
Rotation:

Filename: nn14.all

Channels: Ch1 Ch2 Ch3 Ch4 Ch5 Ch6 Ch7 Plotted: 08:27 Dec 08, 2000

< EMI - ElectroMagnetic Instruments 


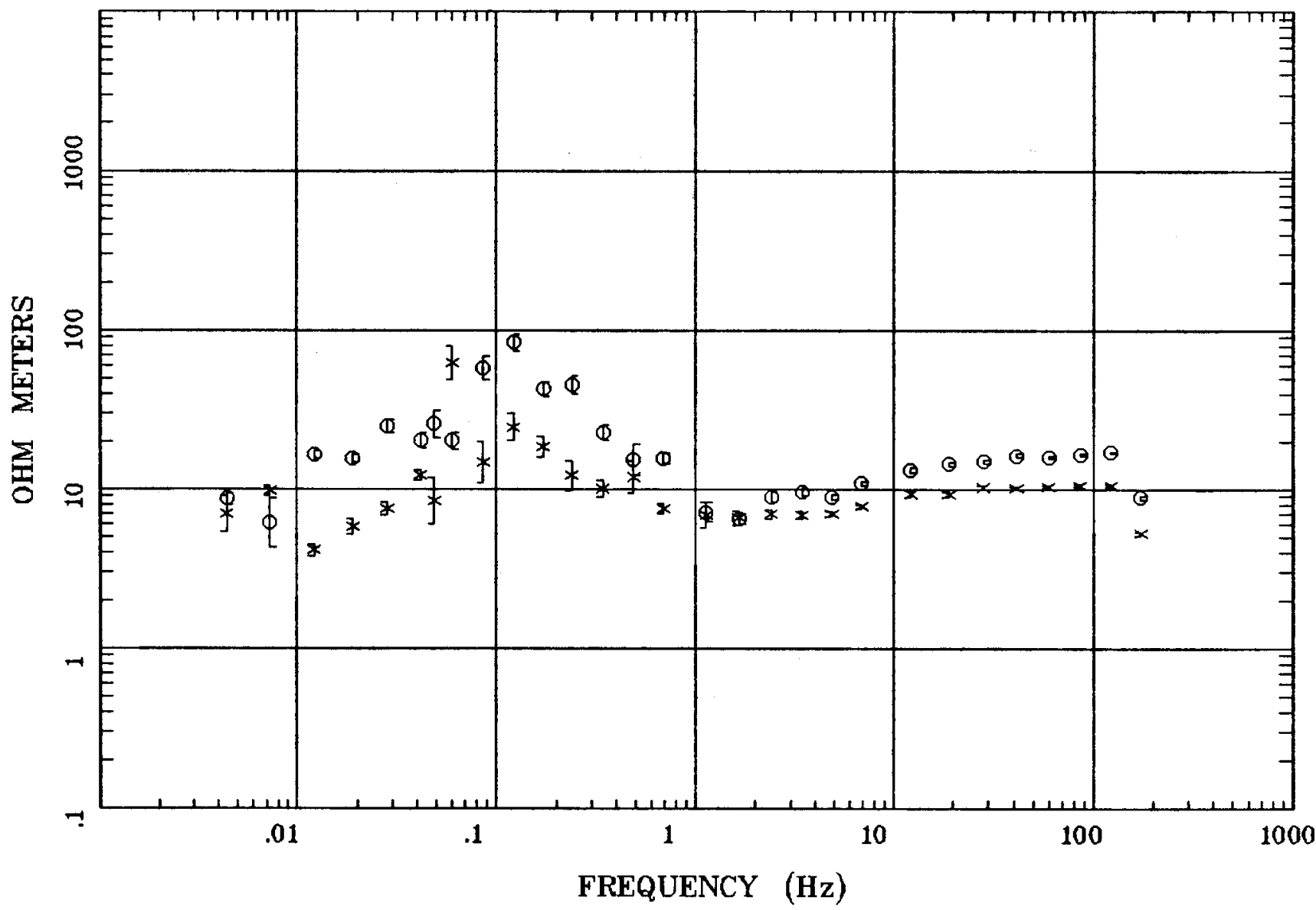

Client:

Remote: $E$ local ref.

Acquired: 09:4 Jul 23, 1997

Survey Co:
Rotation:

Filename: nn 15c.all

Channels: Ch6 Ch7 Ch8 Ch9 Ch10Ch1 Ch2

Plotted: 08:28 Dec 08, 2000

< EMI - ElectroMagnetic Instruments 
Station 15

IMPEDANCE PHASE

...Battle Mtn...

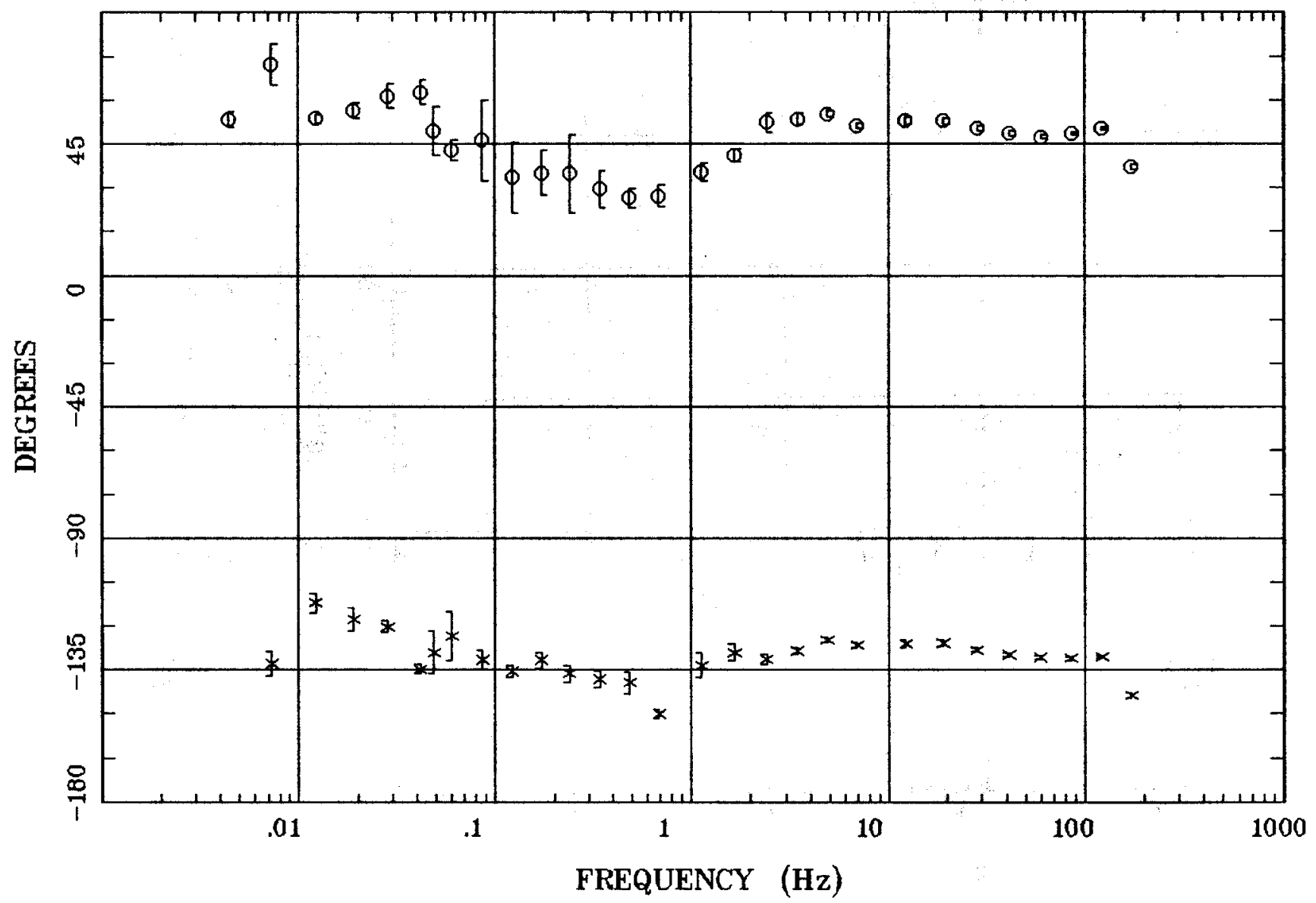

Client:

Remote: E local ref.

Acquired: 09:4 Jul 23, 1997 Survey Co:

Rotation:

Filename: nn 15c.all

Channels: Ch6 Ch7 Ch8 Ch9 Ch10Ch1 Ch2

Plotted: 08:29 Dec 08, 2000

< EMI - ElectroMagnetic Instruments > 


\section{ROTATION ANGLE}

...Battle Mtn...

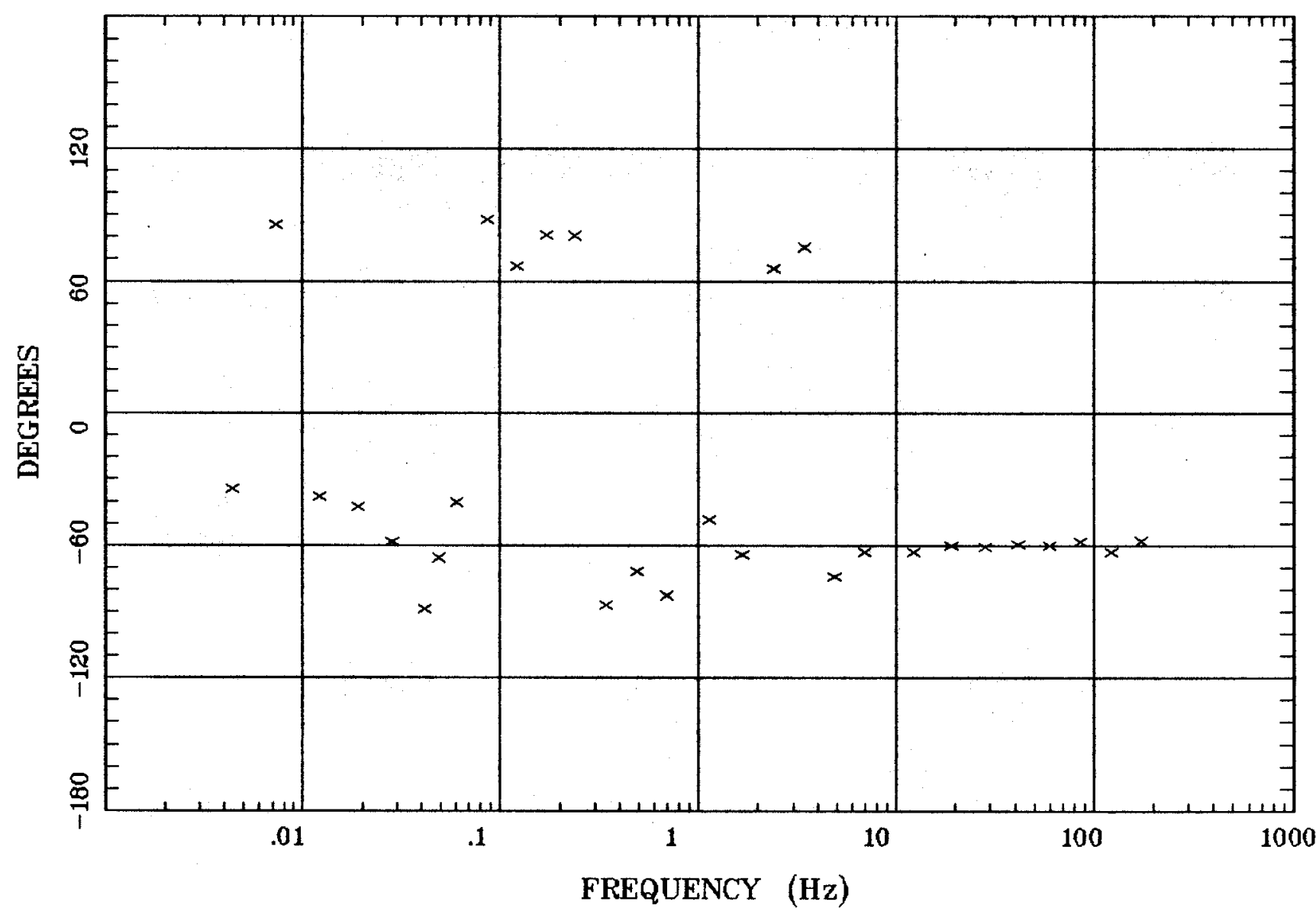

Client:

Remote: E local ref.

Acquired: 09:4 Jul 23, 1997

Survey Co:
Rotation:

Filename: nn 15c.all

Channels: Ch6 Ch7 Ch8 Ch9 Ch10Ch1 Ch2

Plotted: 08:29 Dec 08, 2000

< EMI - ElectroMagnetic Instruments 


\section{IMPEDANCE SKEW}

...Battle Mtn...

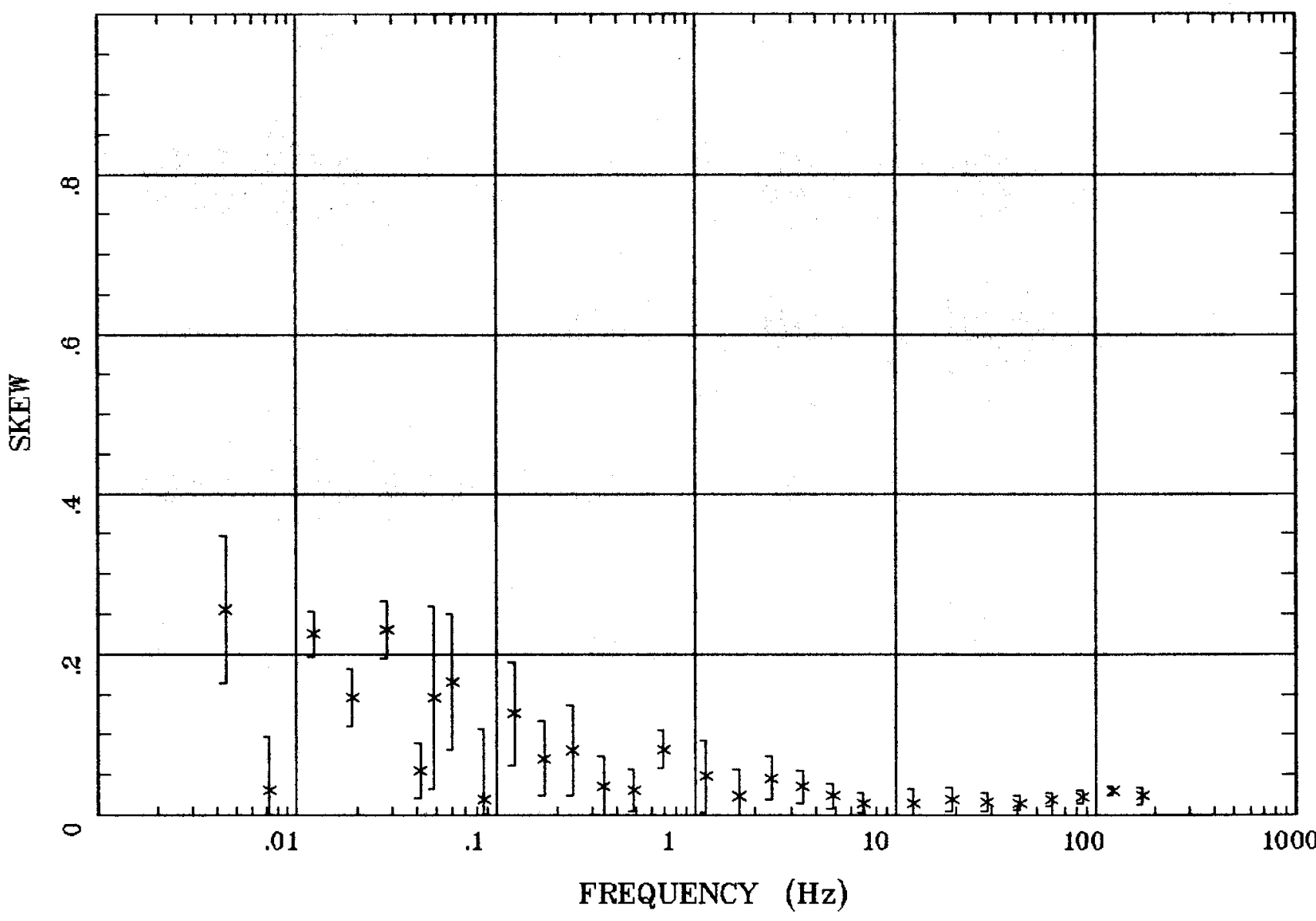

Client:

Remote: E local ref.

Acquired: 09:4 Jul 23, 1997 Survey Co:
Rotation:

Filename: nn15c.all

Channels: Ch6 Ch7 Ch8 Ch9 Ch10Ch1 Ch2 Plotted: 08:29 Dec 08, 2000

$<$ EMI - ElectroMagnetic Instruments 
E MULT Coh.

...Battle Mtn...

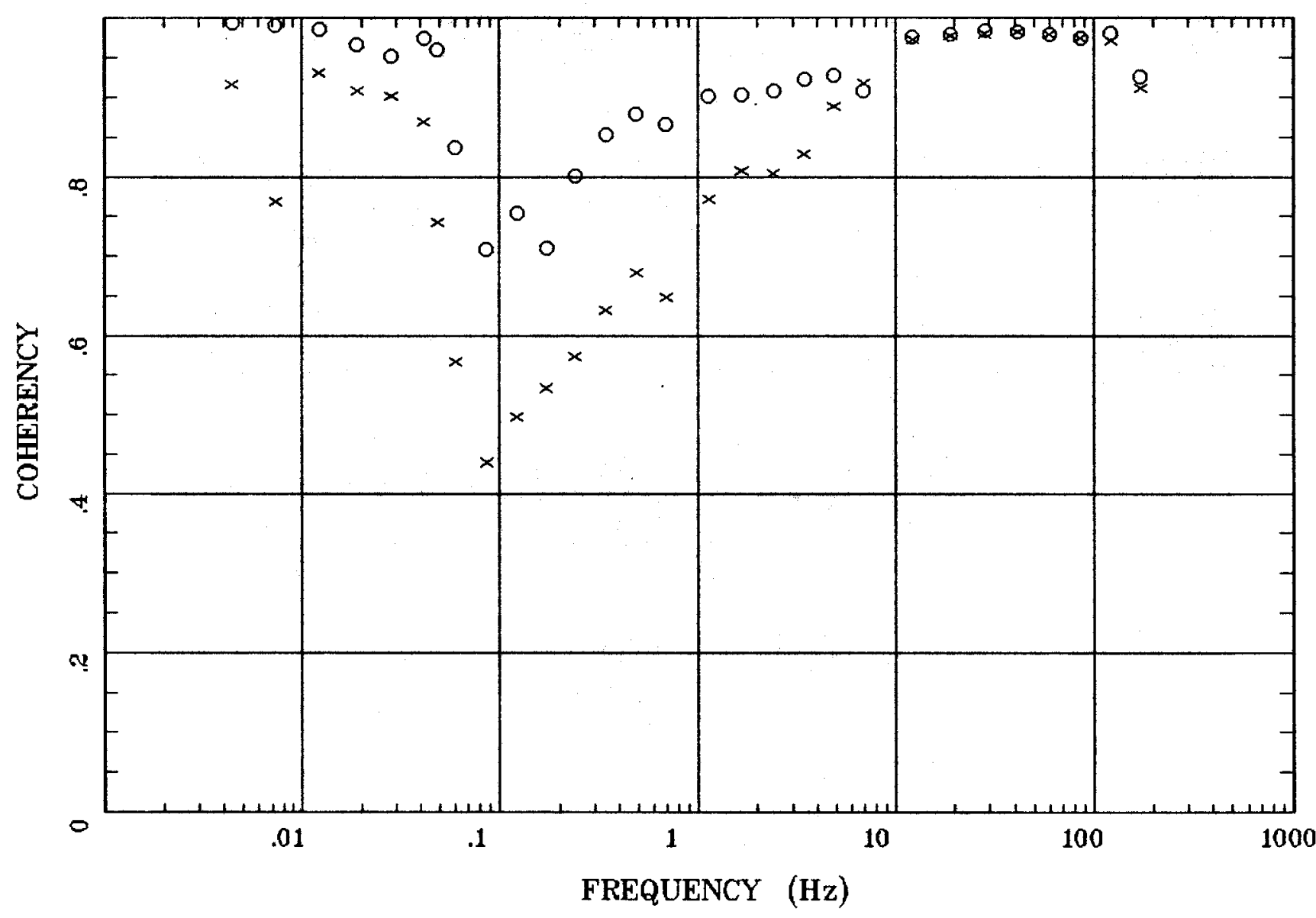

Client:

Remote: E local ref.

Acquired: 09:4 Jul 23, 1997

Survey Co:
Rotation:

Filename: nn 15c.all

Channels: Ch6 Ch7 Ch8 Ch8 Ch10Ch1 Ch2

Plotted: 08:29 Dec 08, 2000

$<$ EMI - ElectroMagnetic Instruments 
POLAR PLOTS

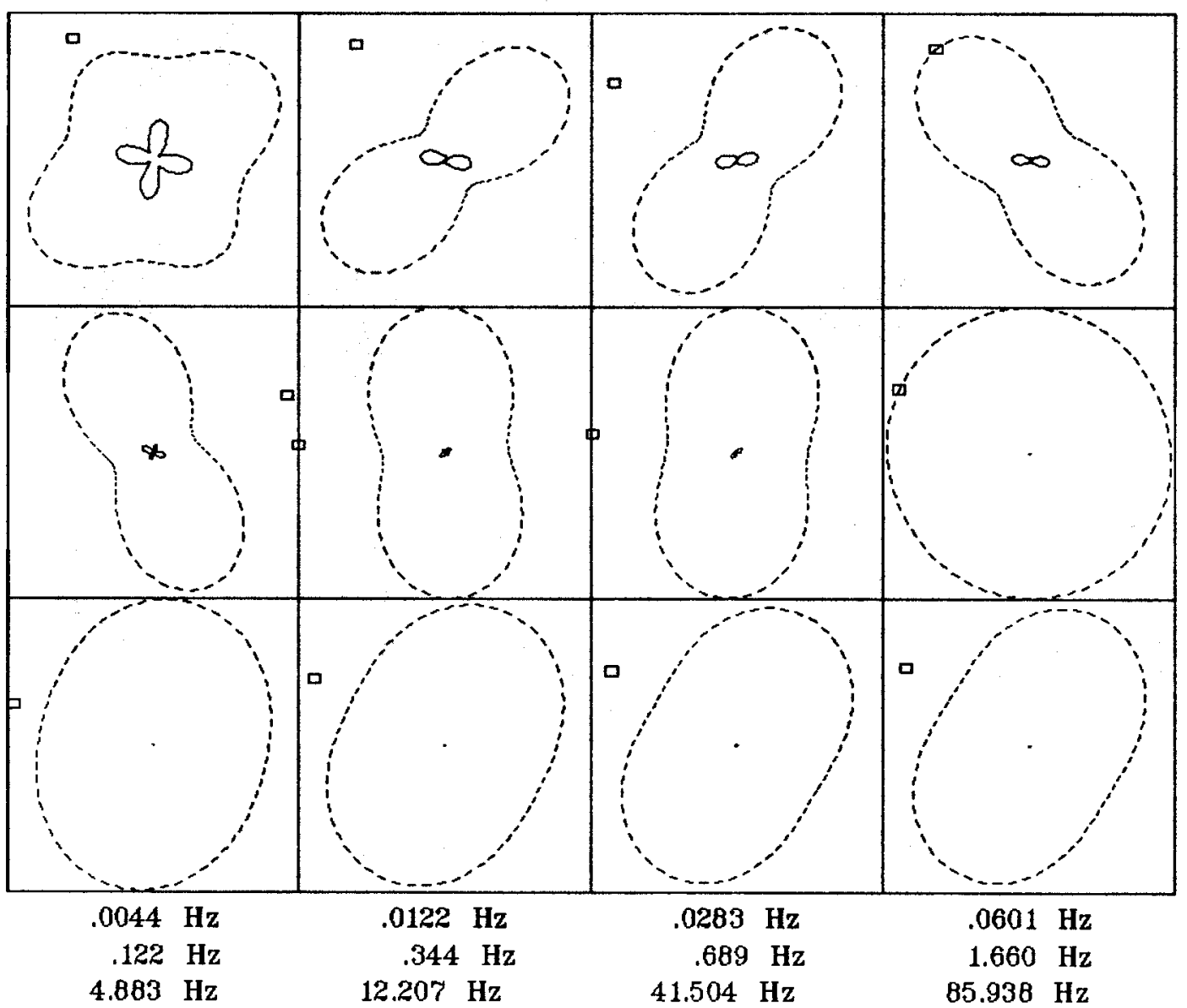

Client:

Remote: E local ref.

Acquired: 09:4 Jul 23, 1997

Survey Co:
Rotation:

...Battle Mtn...

Filename: nn15c.all

Channels: Ch6 Chr Ch8 Ch9 Ch10Ch1 Ch2

Plotted: 08:29 Dec 08, 2000

< EMI - ElectroMagnetic Instruments > 
TIPPER MAGNITUDE

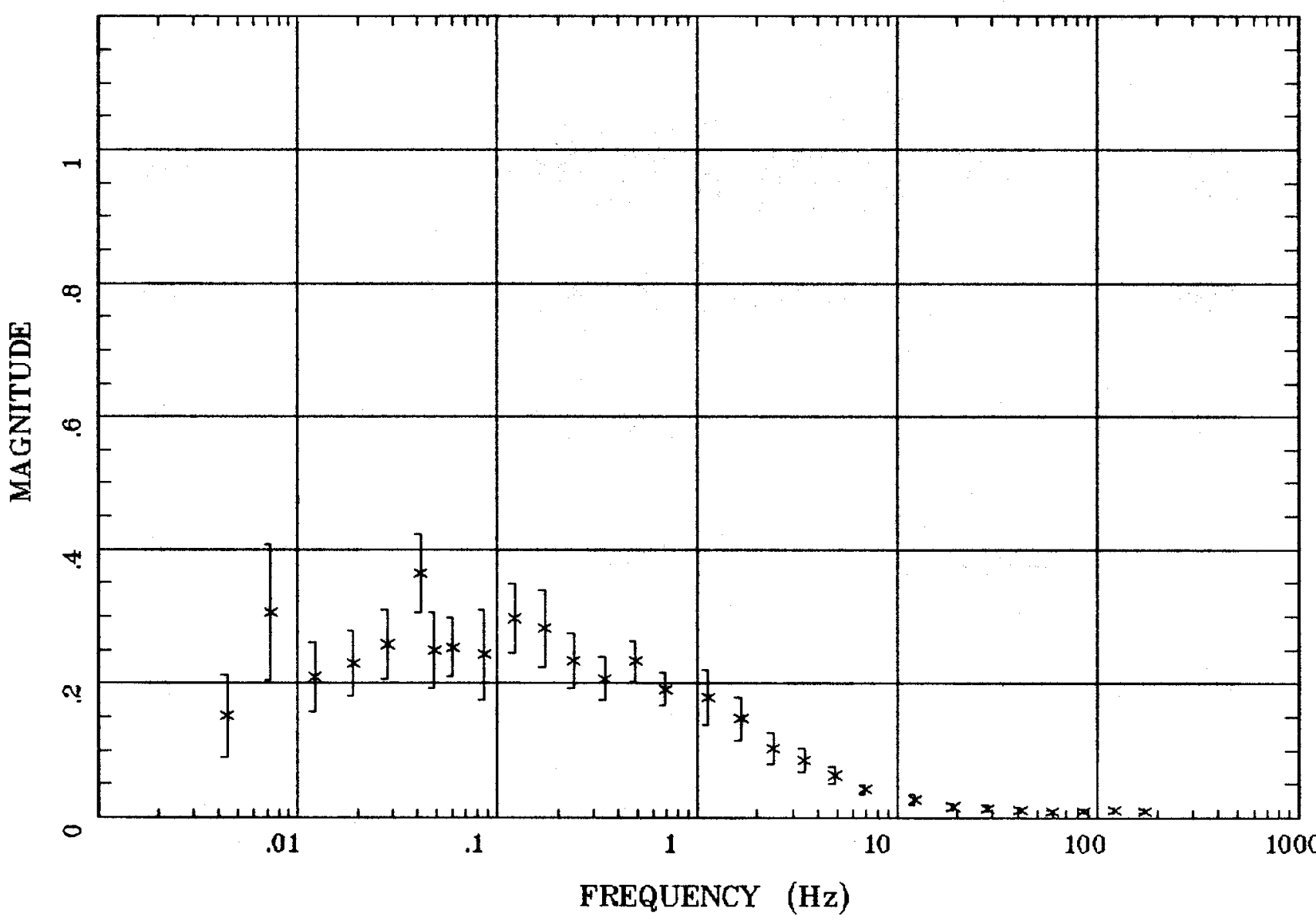

Client:

Remote: E local ref.

Acquired: 09:4 Jul 23, 1997

Survey Co:
Rotation:

Filename: nn 15c.all

Channels: Ch6 Ch7 Ch8 Ch9 Ch10Ch1 Ch2

Plotted: 08:29 Dec 08, 2000

$<$ EMI - ElectroMagnetic Instruments 
TIPPER STRIKE

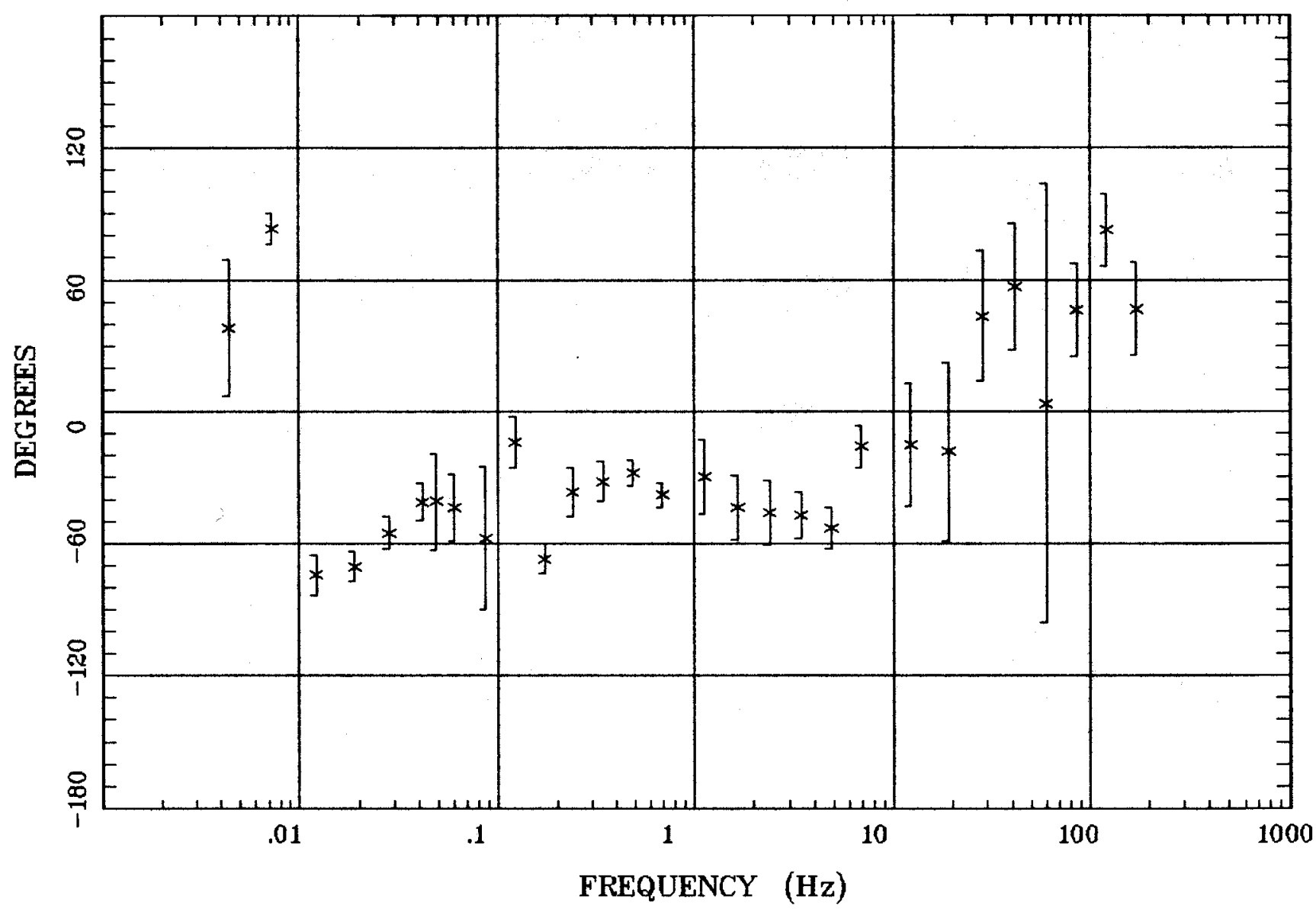

Client:

Remote: E local ref.

Acquired: 09:4 Jul 23, 1997 Survey Co:
Rotation:

Filename: nn15c.all

Channels: Ch6 Ch7 Ch8 Ch9 Ch10Ch1 Ch2 Plotted: 08:29 Dec 08, 2000

< EMI - ElectroMagnetic Instruments 


\section{Station 15}

\section{HzHx.x Coh HzHy.o}

...Battle Mtn...

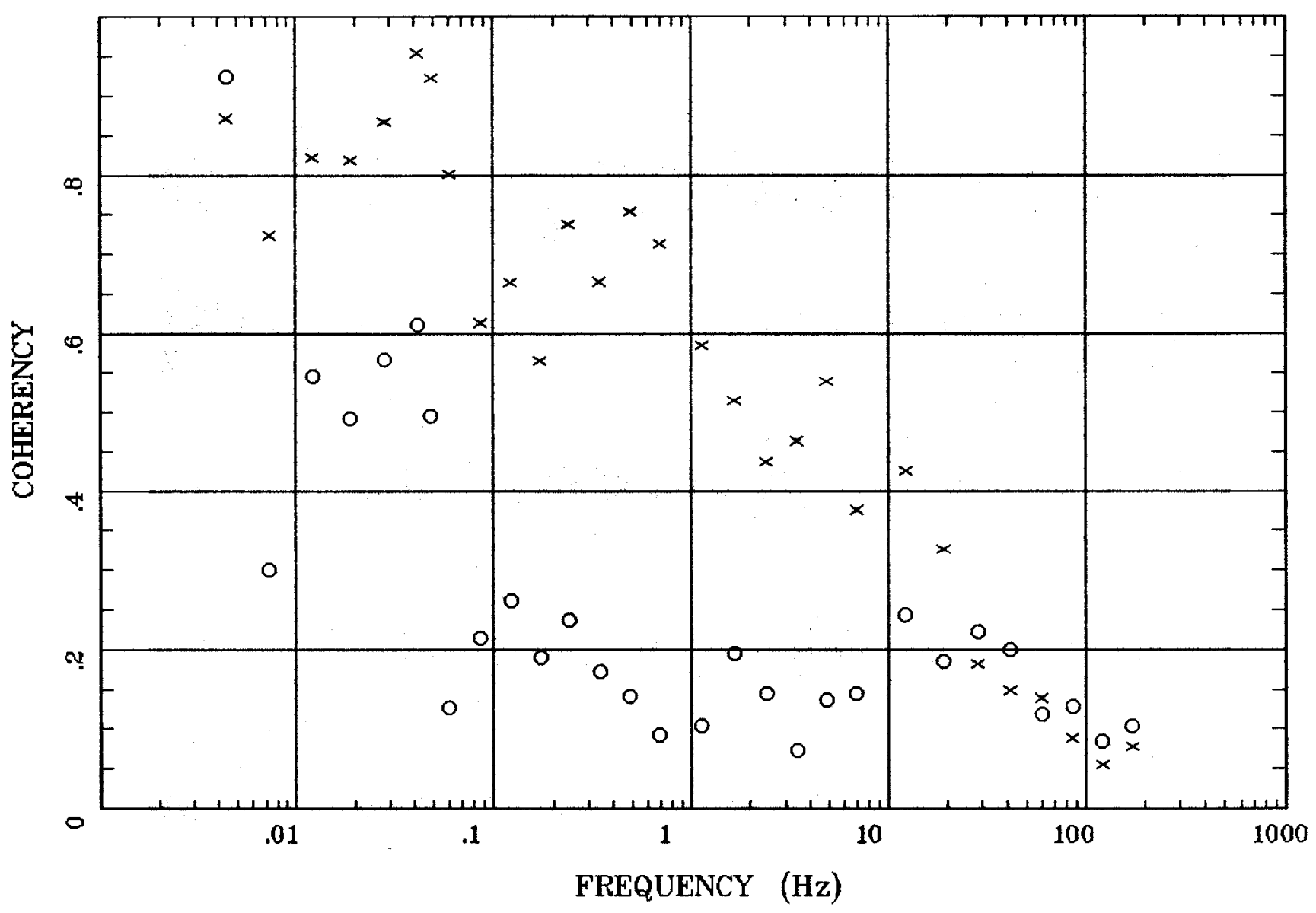

Rotation:

Client:

Filename: nn 15c.all

Remote: E local ref.

Acquired: 09:4 Jul 23, 1997

Channels: Ch6 Ch7 Ch8 Ch9 Ch10Ch1 Ch2

Plotted: 08:29 Dec 08, 2000

Survey Co:

$<$ EMI - ElectroMagnetic Instruments 
APPARENT RESISTIVITY

Battle Mtn.

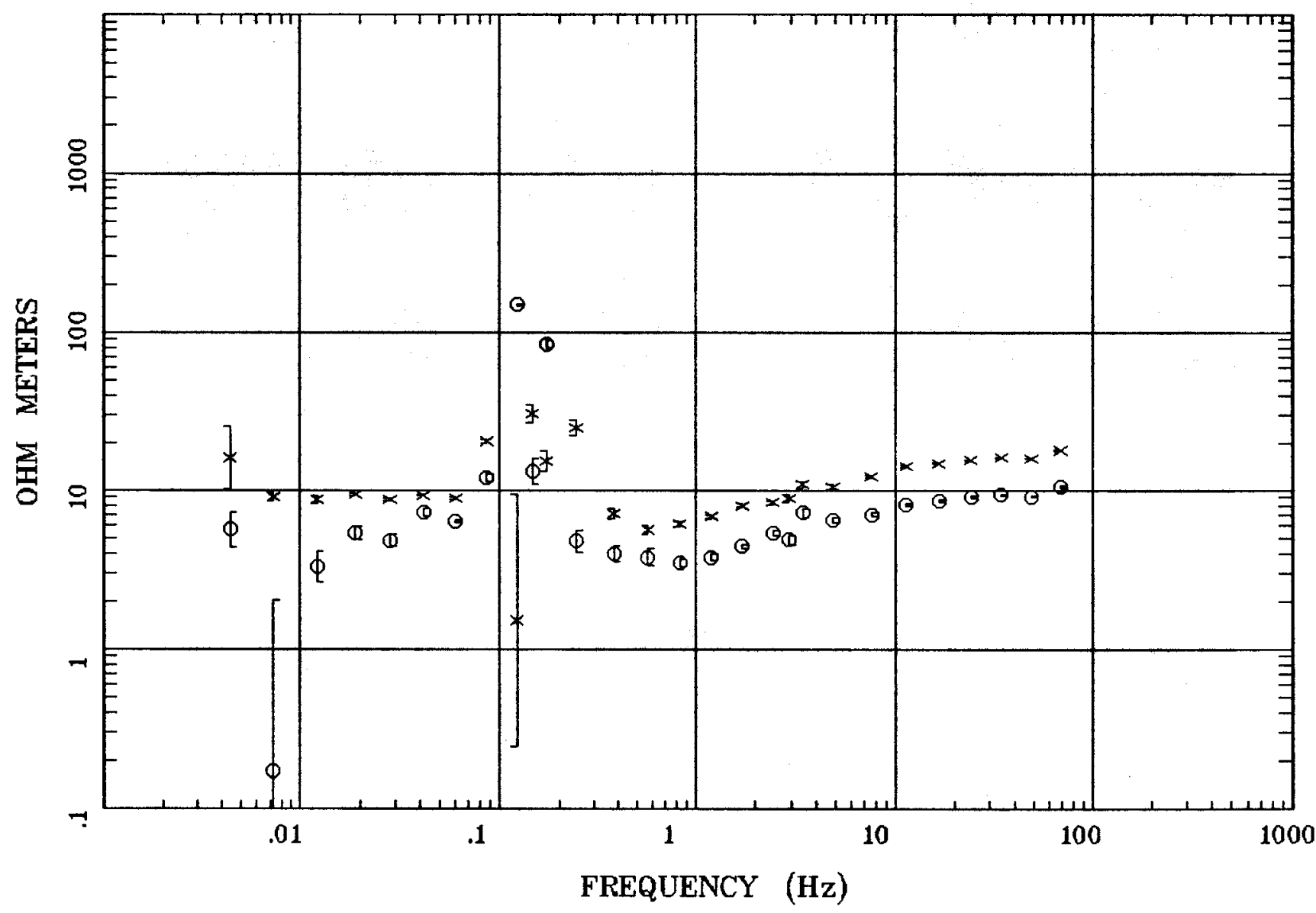

Client:

Remote: e-field local ref Acquired: 15:2 Jul 11, 1997 Survey Co:
Rotation:

Filename: nn16.all

Channels: Ch1 Ch2 Ch3 Ch4 Ch5 Ch6 Ch7 Plotted: 08:30 Dec 08, 2000

< EMI - ElectroMagnetic Instruments 
IMPEDANCE PHASE

Battle Mtn.

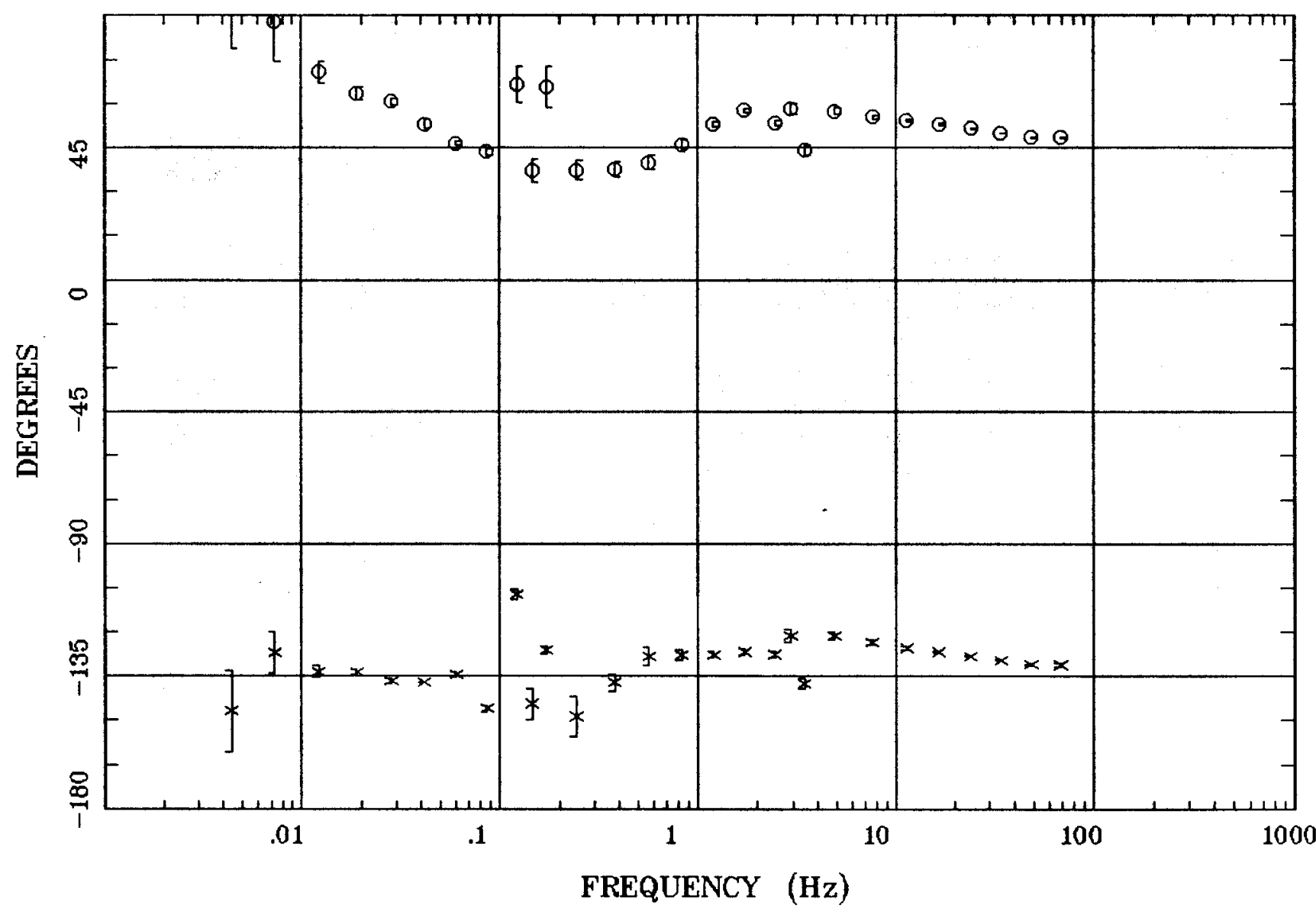

Client:

Remote: e-field local ref

Acquired: 15:2 Jul 11, 1997

Survey Co:
Rotation:

Filename: nn16.all

Channels: Ch1 Ch2 Ch3 Ch4 Ch5 Ch6 Ch7

Plotted: 08:30 Dec 08, 2000

< EMI - ElectroMagnetic Instruments 
Station 16

ROTATION ANGLE

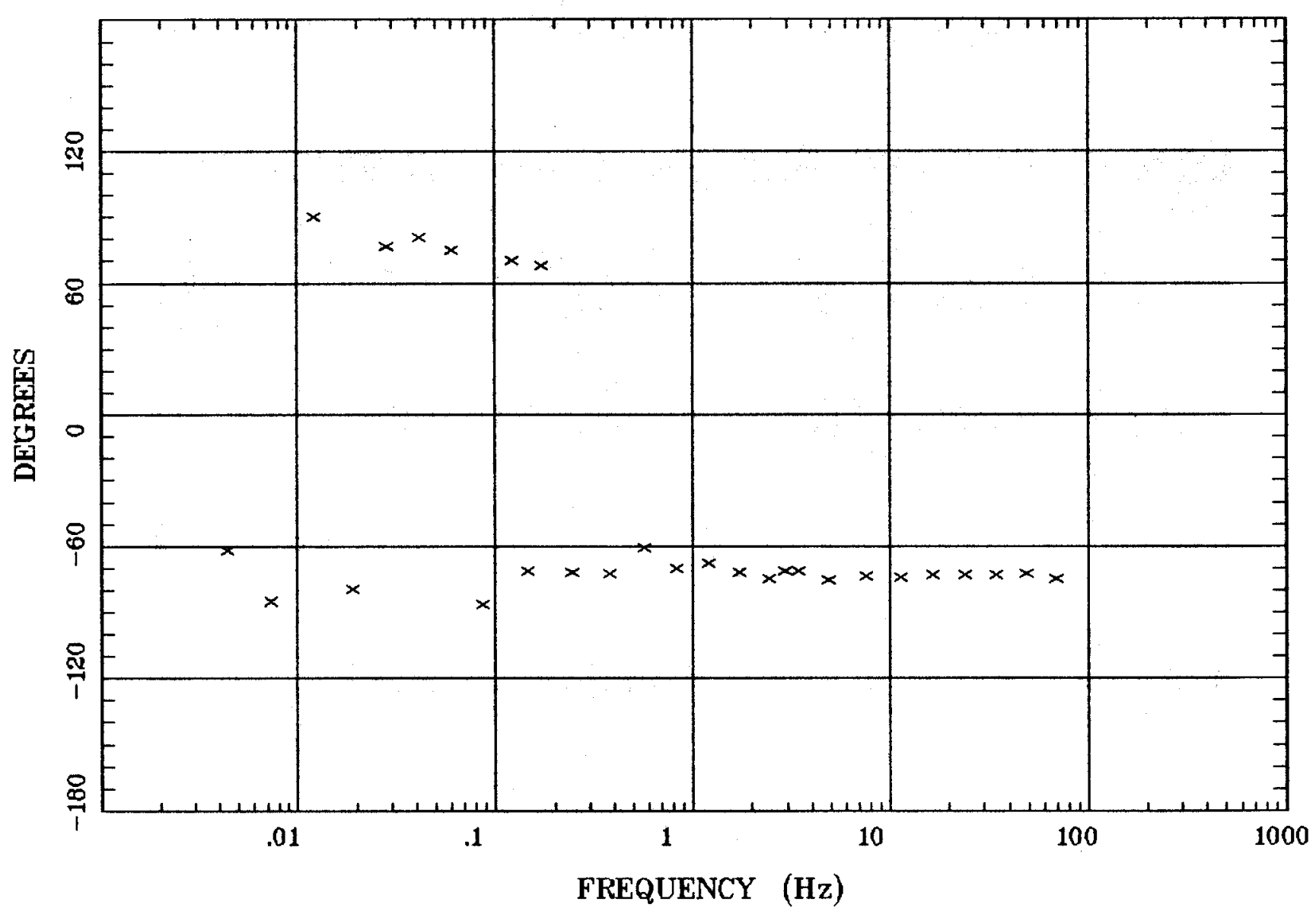

Client:

Remate: e-field local ref

Acquired: 15:2 Jul 11, 1997

Survey Co:
Battle Mtn.

Rotation:

Filename: nn16.all

Channels: Ch1 Ch2 Ch3 Ch4 Ch5 Ch6 Ch7

Plotted: 08:30 Dec 08, 2000

\& EMI - ElectroMagnetic Instruments > 


\section{IMPEDANCE SKEW}

Battle Mtn.

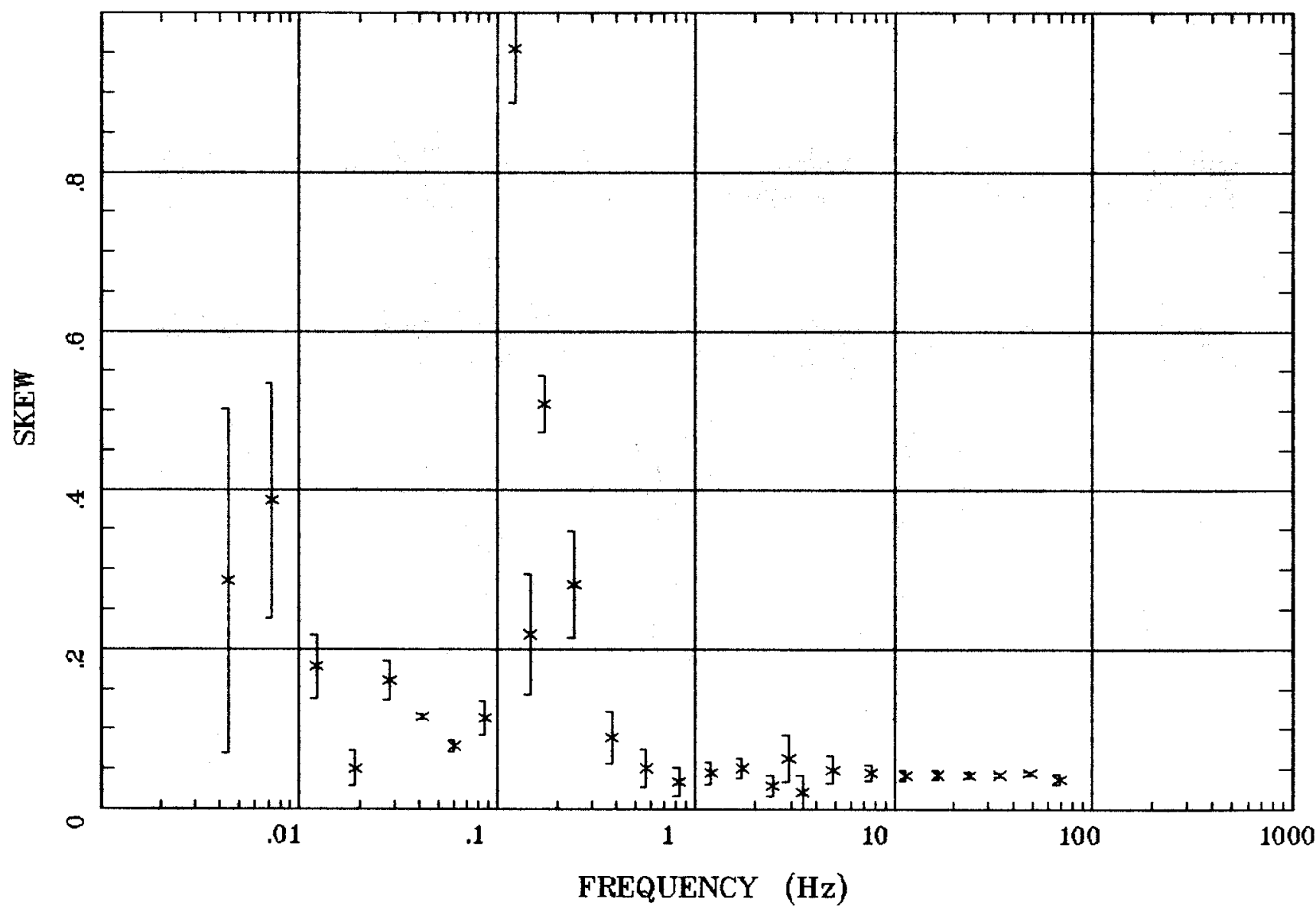

Client:

Remote: e-field local ref

Acquired: 15:2 Jul 11, 1997

Survey Co:
Rotation:

Filename: nn16.all

Channels: Ch1 Ch2 Ch3 Ch4 Ch5 Ch6 Ch7

Plotted: 08:30 Dec 08, 2000

$<$ EMI - ElectroMagnetic Instruments 
E MULT Coh.

Battle Mtn.

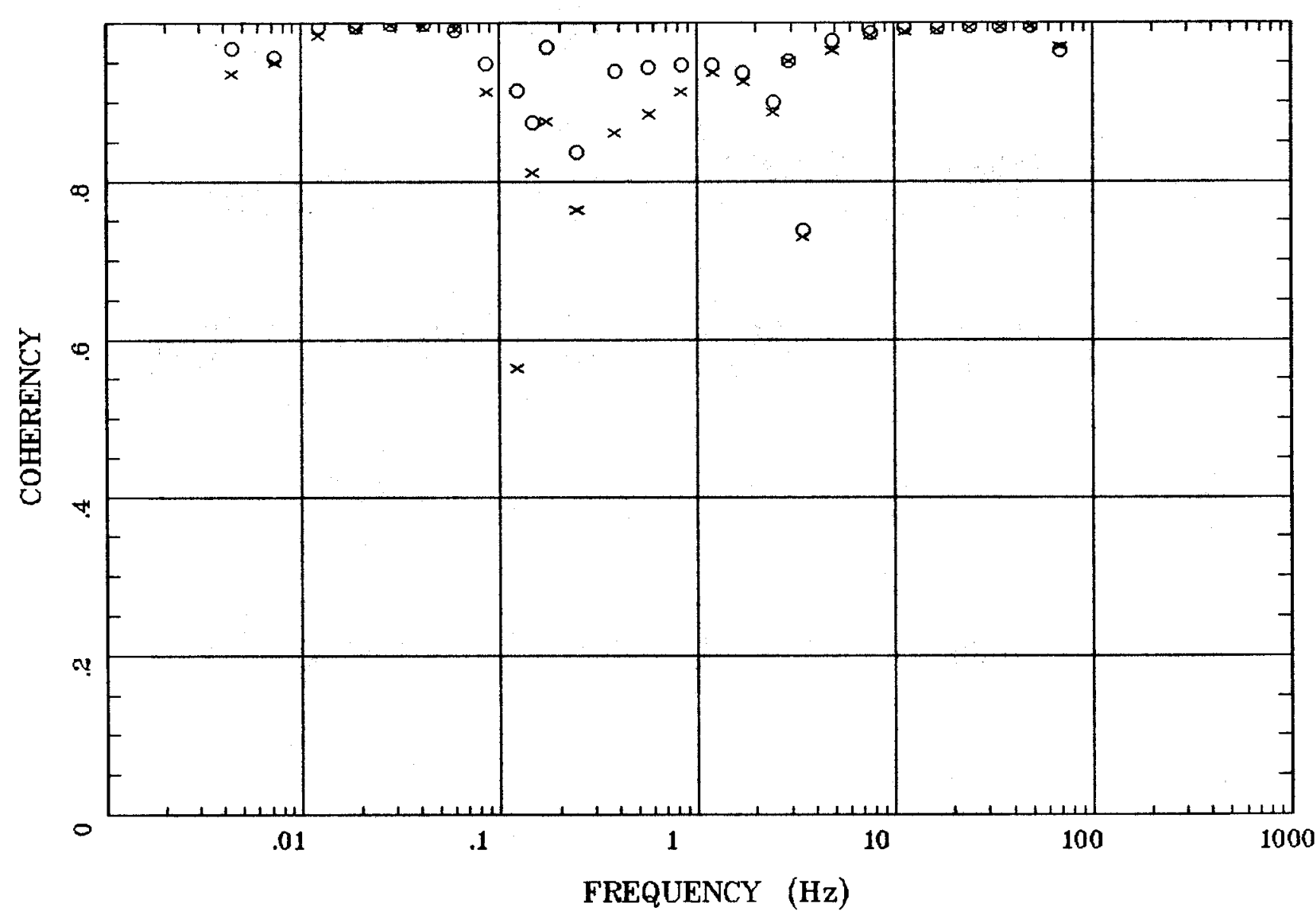

Client:

Remote: e-field local ref Acquired: $15: 2$ Jul 11, 1997 Survey Co:
Rotation:

Filename: nn16.all

Channels: Ch1 Ch2 Ch3 Ch4 Ch5 Ch6 Ch7 Plotted: 08:30 Dec 08, 2000

< EMI - ElectroMagnetic Instruments 
POLAR PLOTS

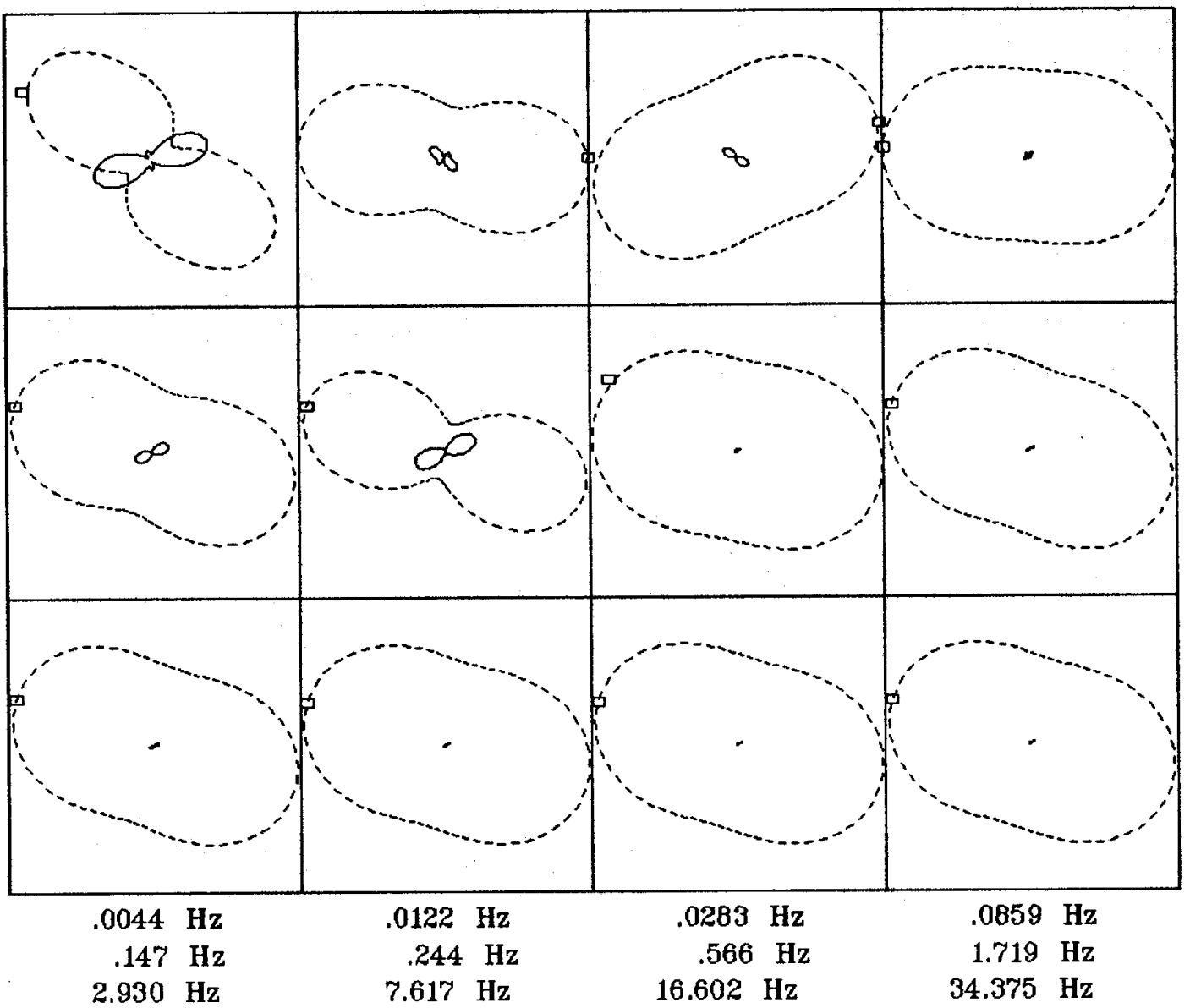

Client:

Remote: e-field local ref Acquired: $15: 2$ Jul 11, 1997 Survey Co:
Battle Mtn.

Rotation:

Filename: nn16.all

Channels: Ch1 Ch2 Ch3 Ch4 Ch5 Ch6 Ch7

Plotted: 08:30 Dec 08, 2000

< EMI - ElectroMagnetic Instruments 


\section{TIPPER MAGNITUDE}

Battle Mtn.

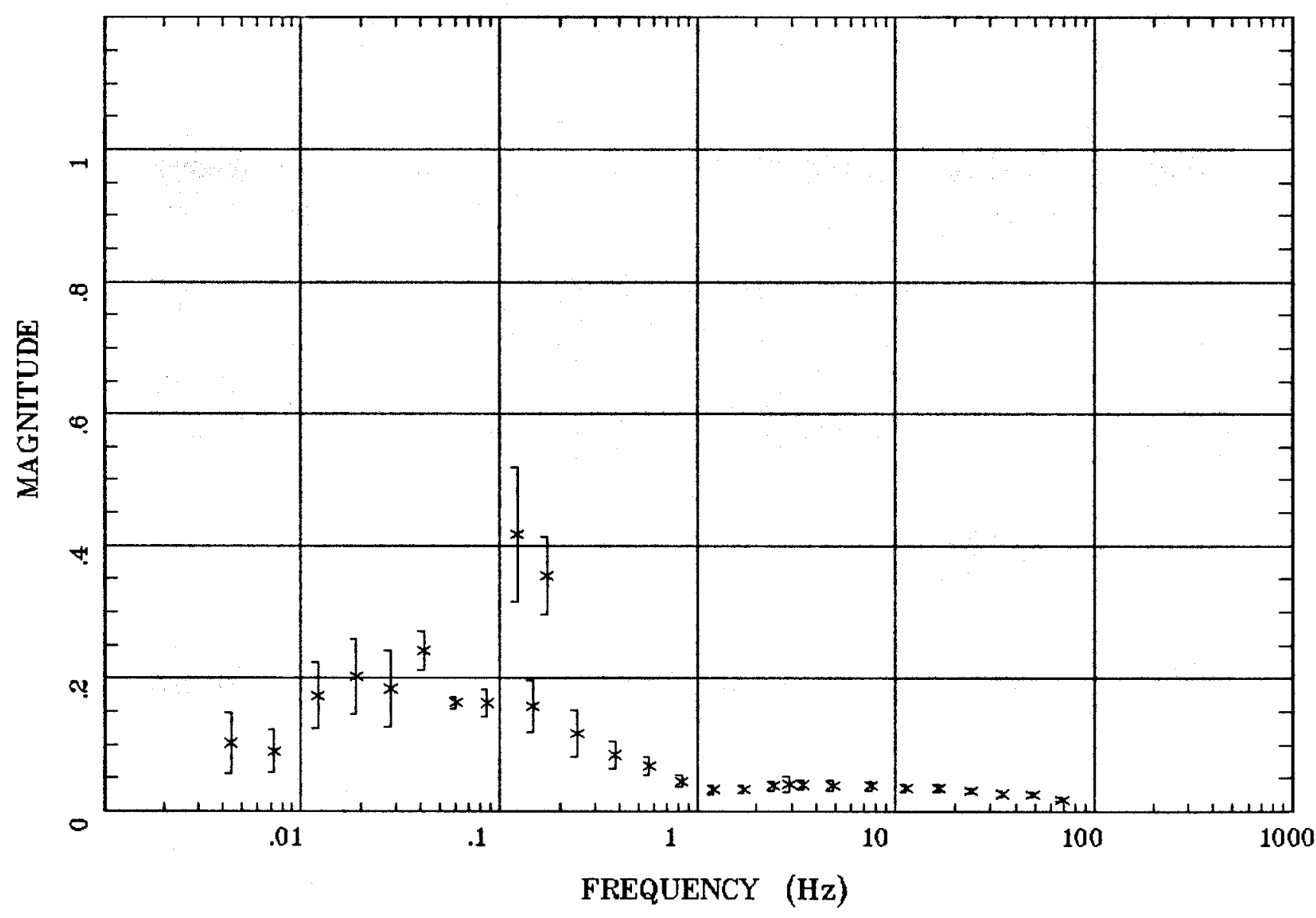

Client:

Remote: e-field local ref

Acquired: 15:2 Jul 11, 1997

Survey Co:
Rotation:

Filename: nn16.all

Channels: Ch1 Ch2 Ch3 Ch4 Ch5 Ch6 Ch7

Plotted: 08:30 Dec 08, 2000

$<$ EMI - ElectroMagnetic Instruments > 
TIPPER STRIKE

Battle Mtn.

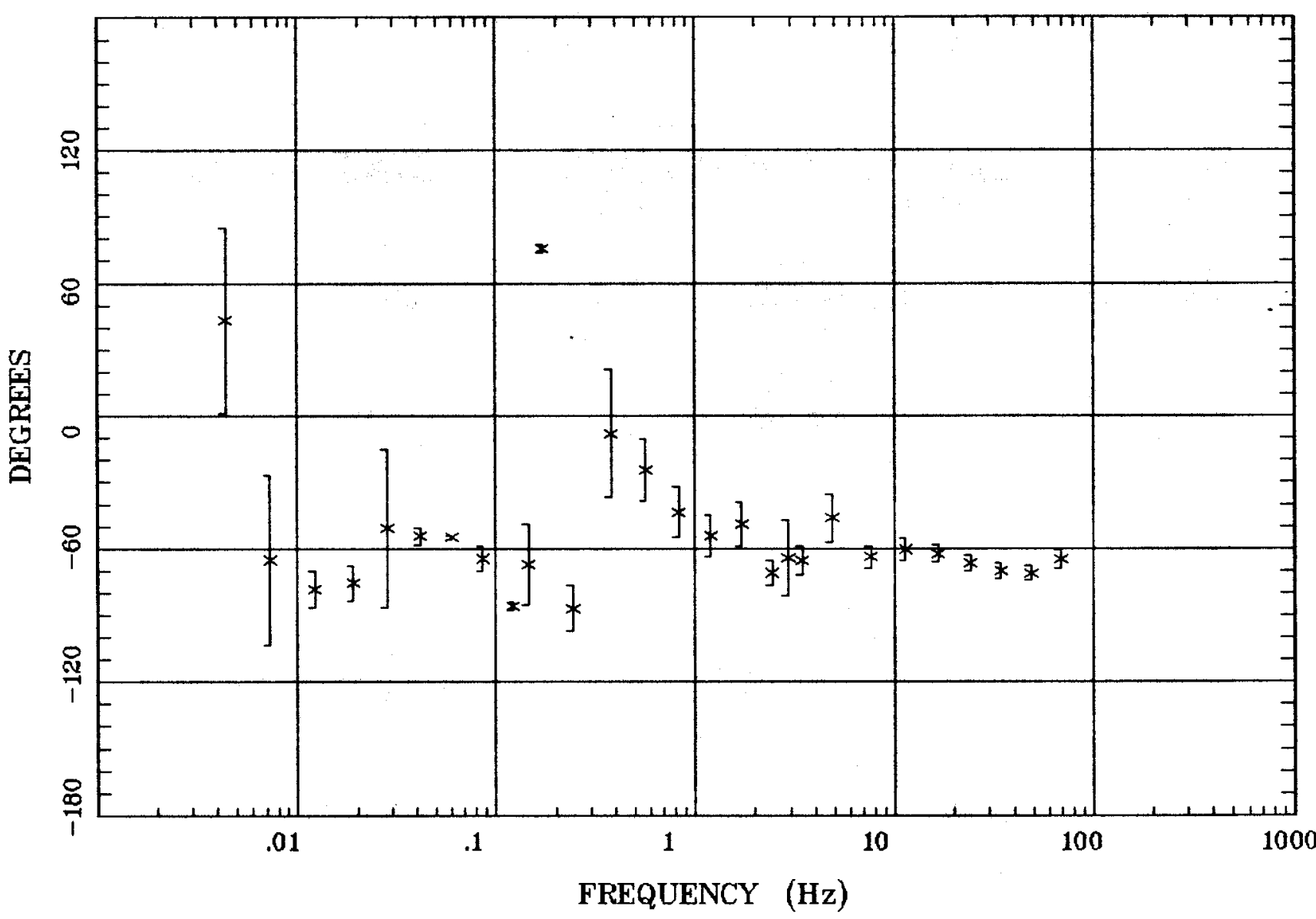

Client:

Remote: e-field locel ref Acquired: 15:2 Jul 11, 1997 Survey Co:

Rotation:

Filename: nn16.all

Channels: Ch1 Ch2 Ch3 Ch4 Ch5 Ch6 Ch7

Plotted: 08:30 Dec 08, 2000

$<$ EMI - ElectroMagnetic Instruments 
HzHx.x Coh HzHy.o

Battle Mtn.

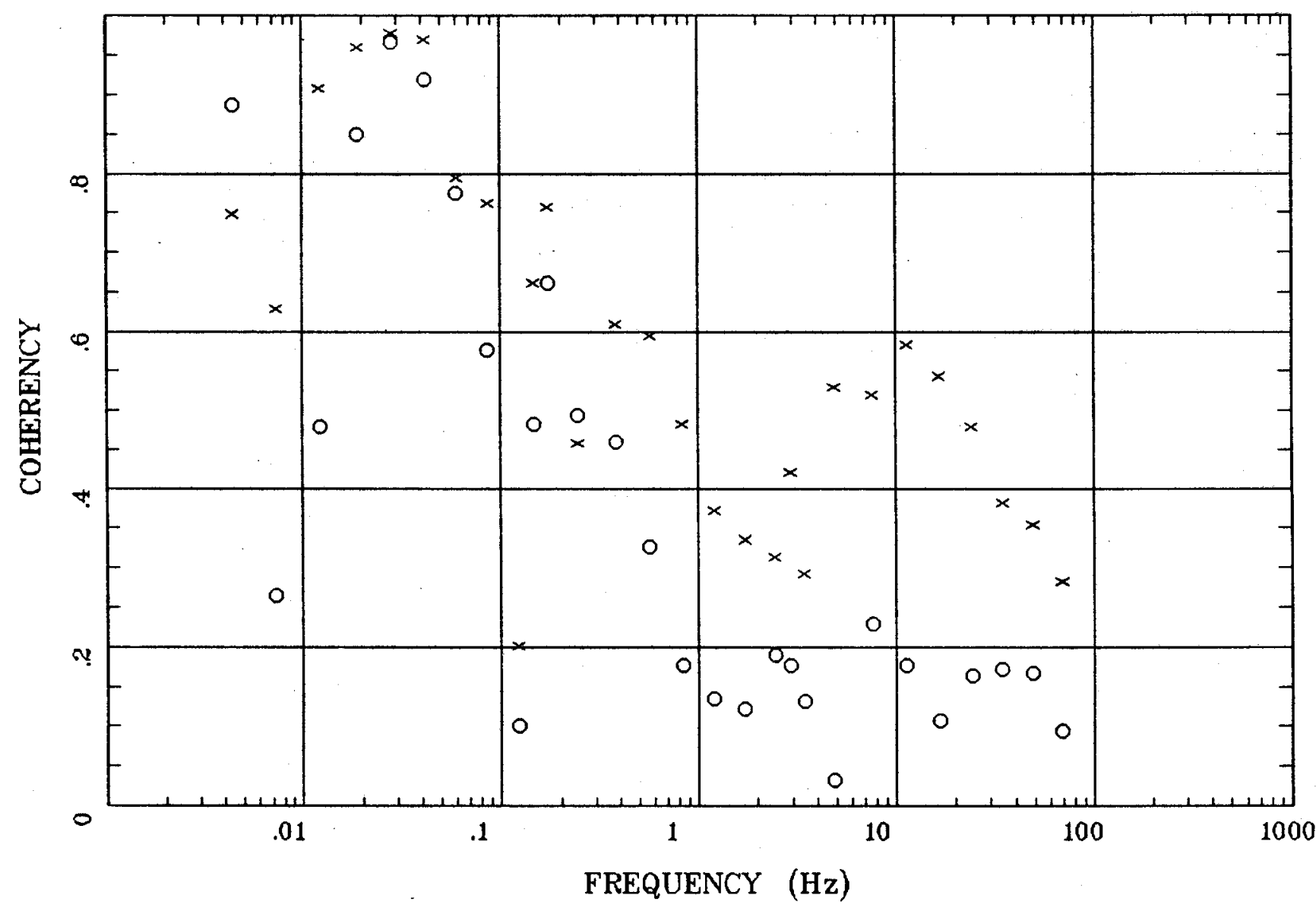

Client:

Remote: e-field local ref Acquired: 15:2 Jul 11, 1997 Survey Co:
Rotation:

Filename: nn16.all

Channels: Ch1 Ch2 Ch3 Ch4 Ch5 Ch6 Ch7 Plotted: 08:30 Dec 08, 2000

< EMI - ElectroMagnetic Instruments 


\section{APPARENT RESISTIVITY}

Battle Mtn.

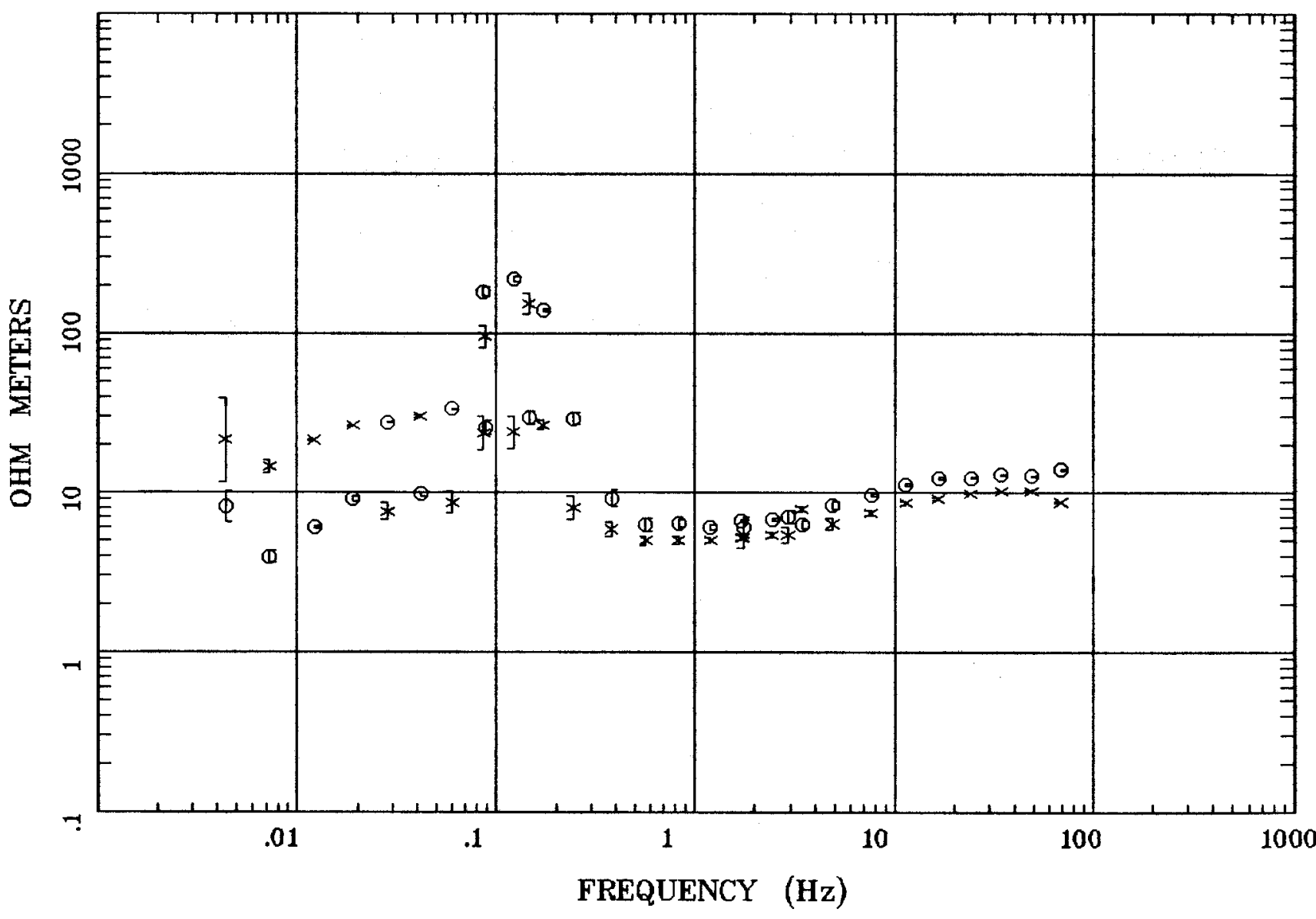

Client:

Remote: e-field local ref Acquired: 12:1 Jul 11, 1997 Survey Co:
Rotation:

Filename: nn19.all

Channels: Ch1 Ch2 Ch3 Ch4 Ch5 Ch6 Ch7 Plotted: 08:31 Dec 08, 2000

$<$ EMI - ElectroMagnetic Instruments 


\section{Station 19}

IMPEDANCE PHASE

Battle Mtn.

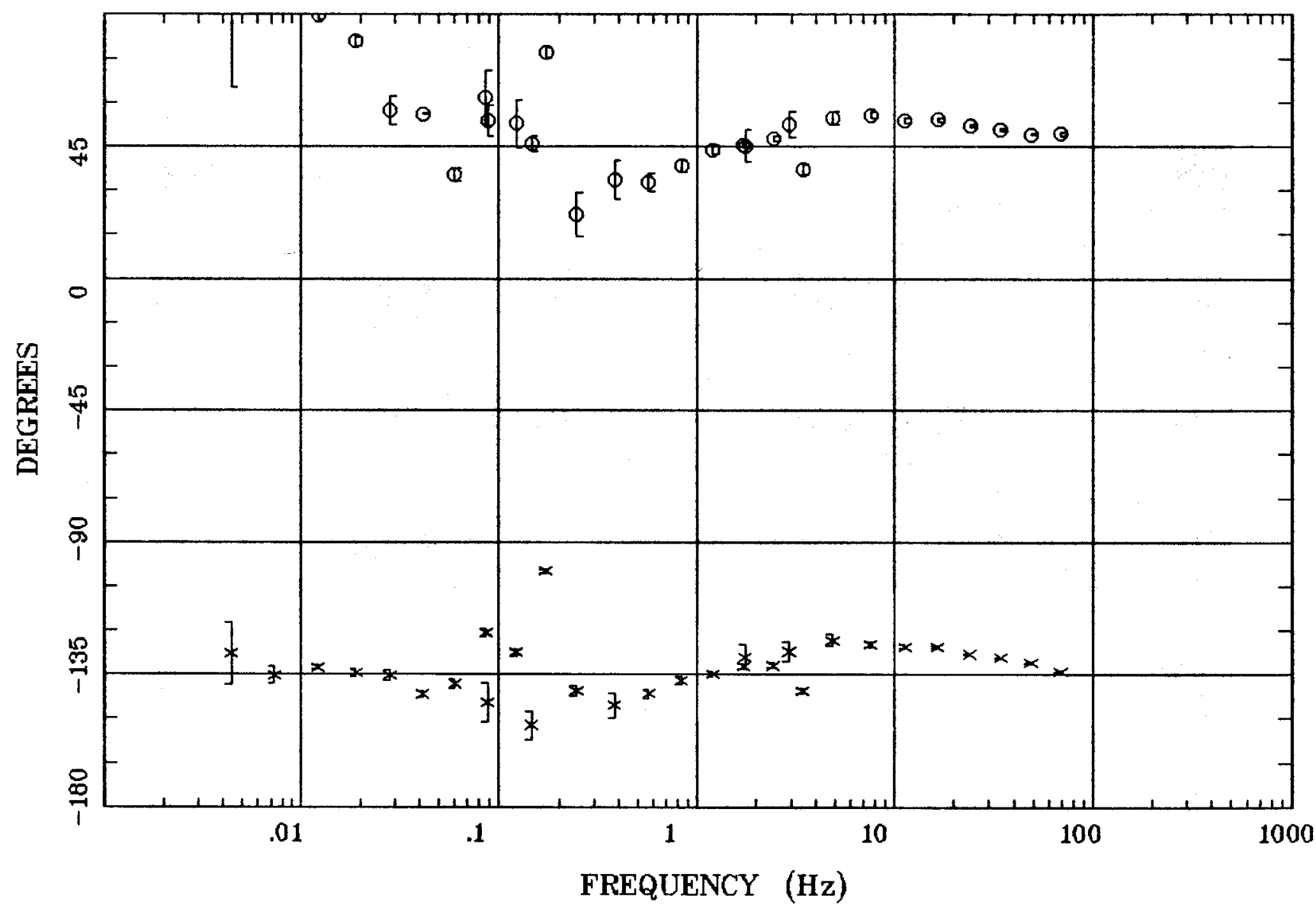

Client:

Remote: e-field local ref

Acquired: 12:1 Jul 11, 1997 Survey Co:
Rotation:

Filename: nn19.all

Channels: Ch1 Ch2 Ch3 Ch4 Ch5 Ch6 Ch7 Plotted: 08:31 Dec 08, 2000

$<$ EMI - ElectroMagnetic Instruments 


\section{ROTATION ANGLE}

Battle Mtn.

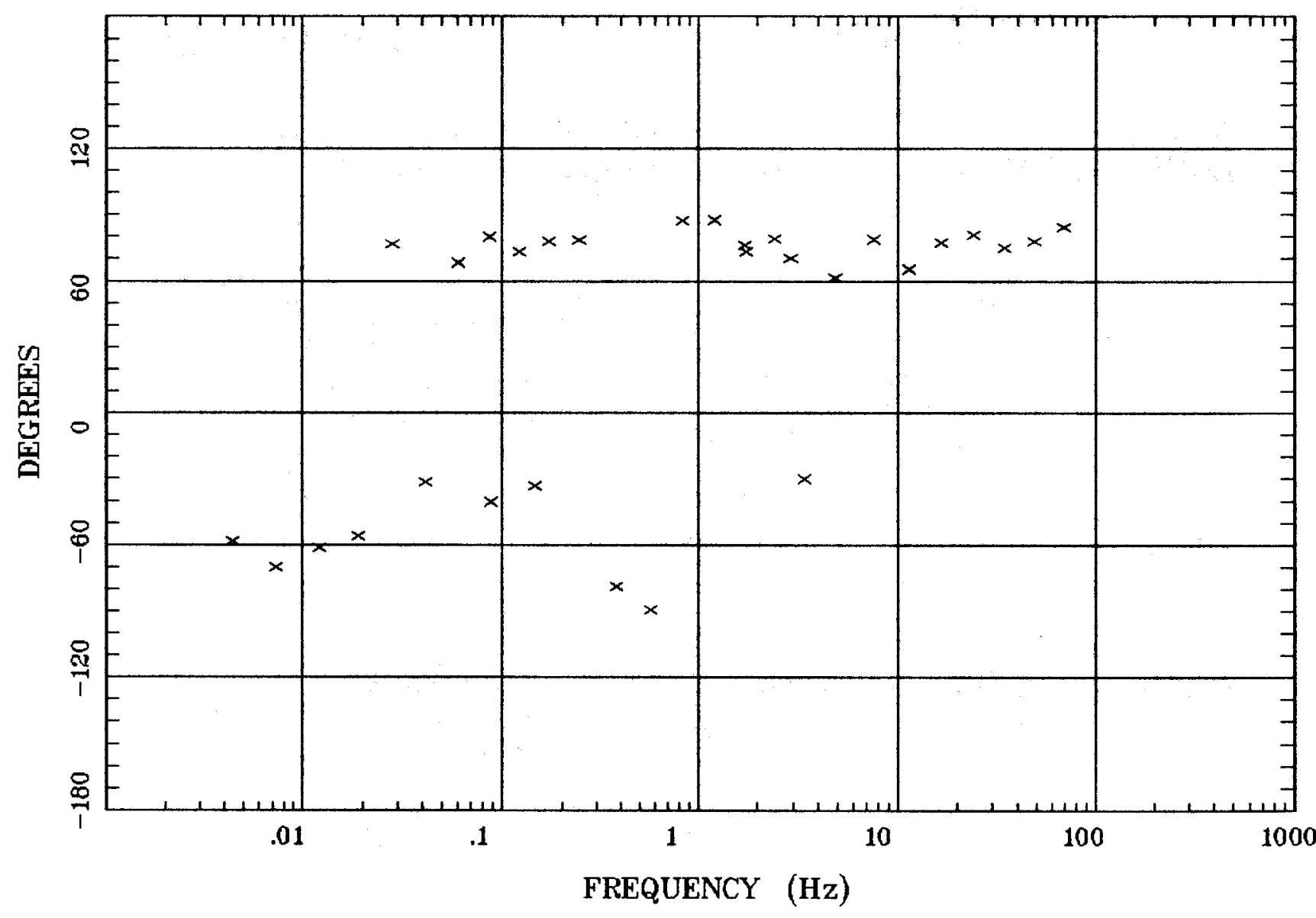

Client:

Remote: e-field local ref Acquired: 12:1 Jul 11, 1997 Survey Co:
Rotation:

Filename: nn19.all

Channels: Ch1 Ch2 Ch3 Ch4 Ch5 Ch6 Ch7 Plotted: 08:31 Dec 08, 2000

$<$ EMI - ElectroMagnetic Instruments > 


\section{Station 19}

IMPEDANCE SKEW

Battle Mtn.

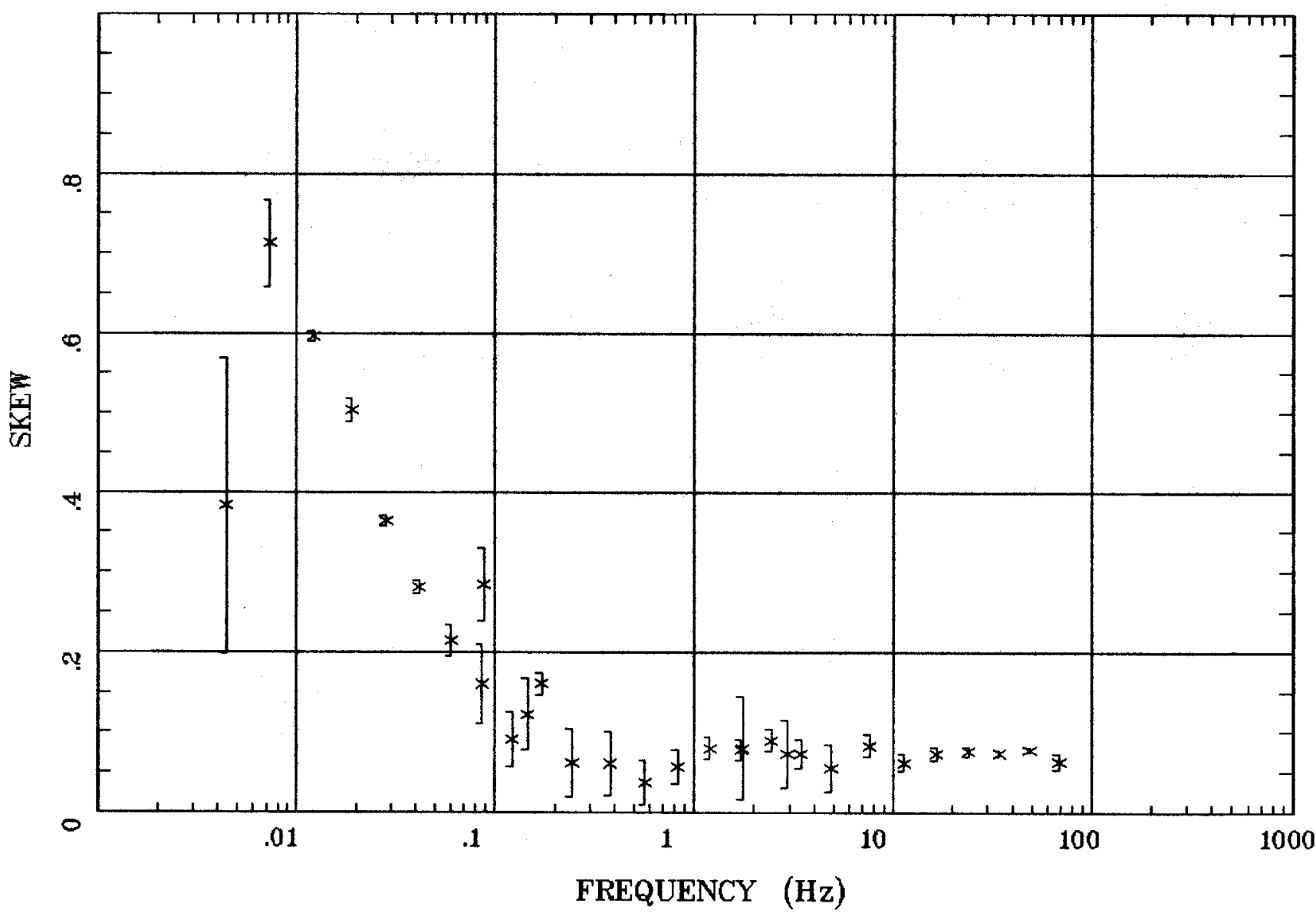

Client:

Remote: e-field local ref Acquired: $12: 1 \mathrm{Jul} \mathrm{11,1997}$ Survey Co:
Rotation:

Filename: nn19.all

Channels: Ch1 Ch2 Ch3 ch4 Ch5 Ch6 Ch7

Plotted: 08:31 Dec 08, 2000

$<$ EMI - ElectroMagnetic Instruments > 


\section{Station 19}

E MULT Coh.

Battle Mtn.

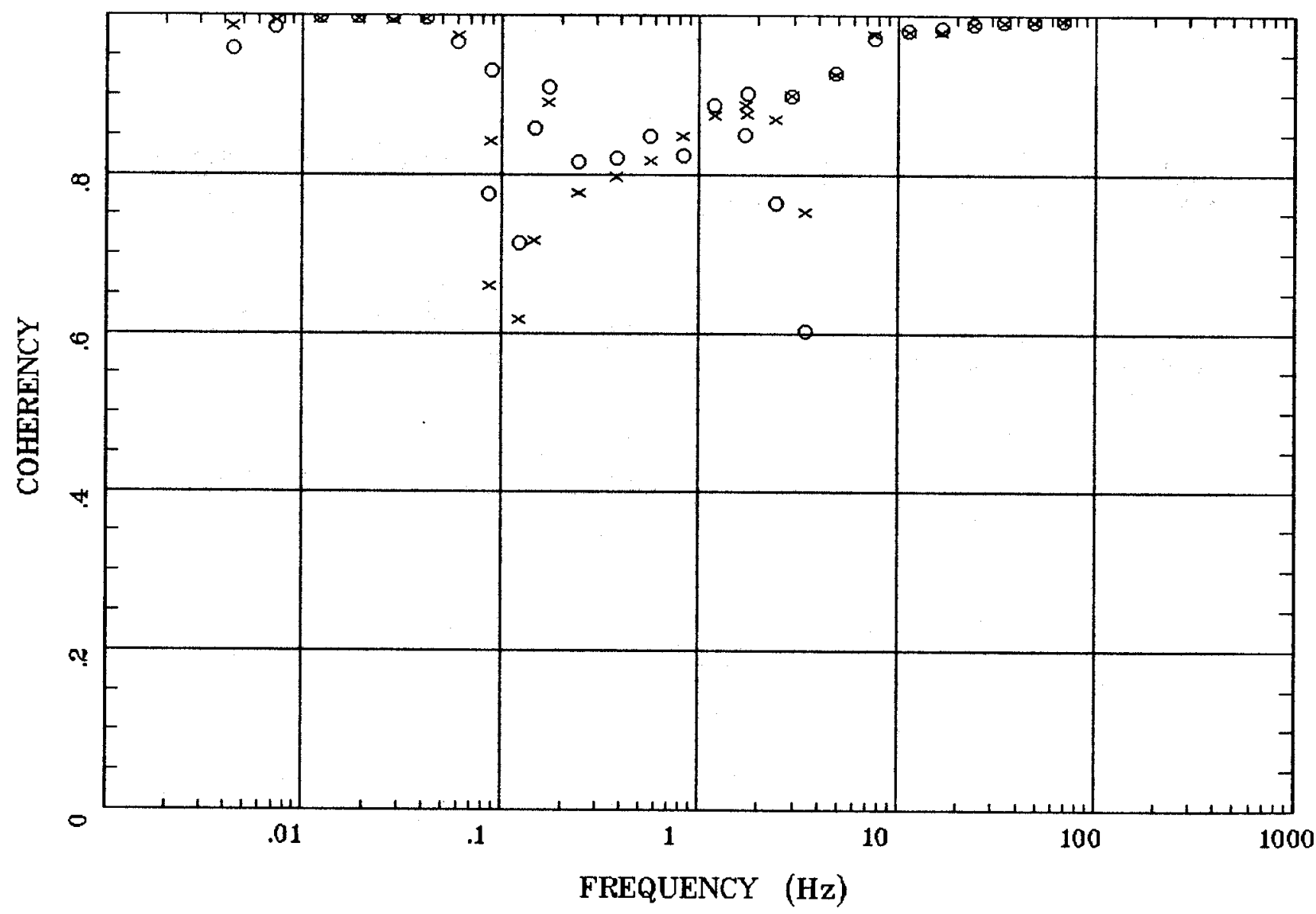

Client:

Remote: e-field local ref Acquired: 12:1 Jul 11, 1997 Survey Co:
Rotation:

Filename: nn19.all

Channels: Ch1 Ch2 Ch3 Ch4 Ch5 Ch6 Ch7 Plotted: 08:31 Dec 08, 2000

$<$ EMI - ElectroMagnetic Instruments 
Battle Mtn.

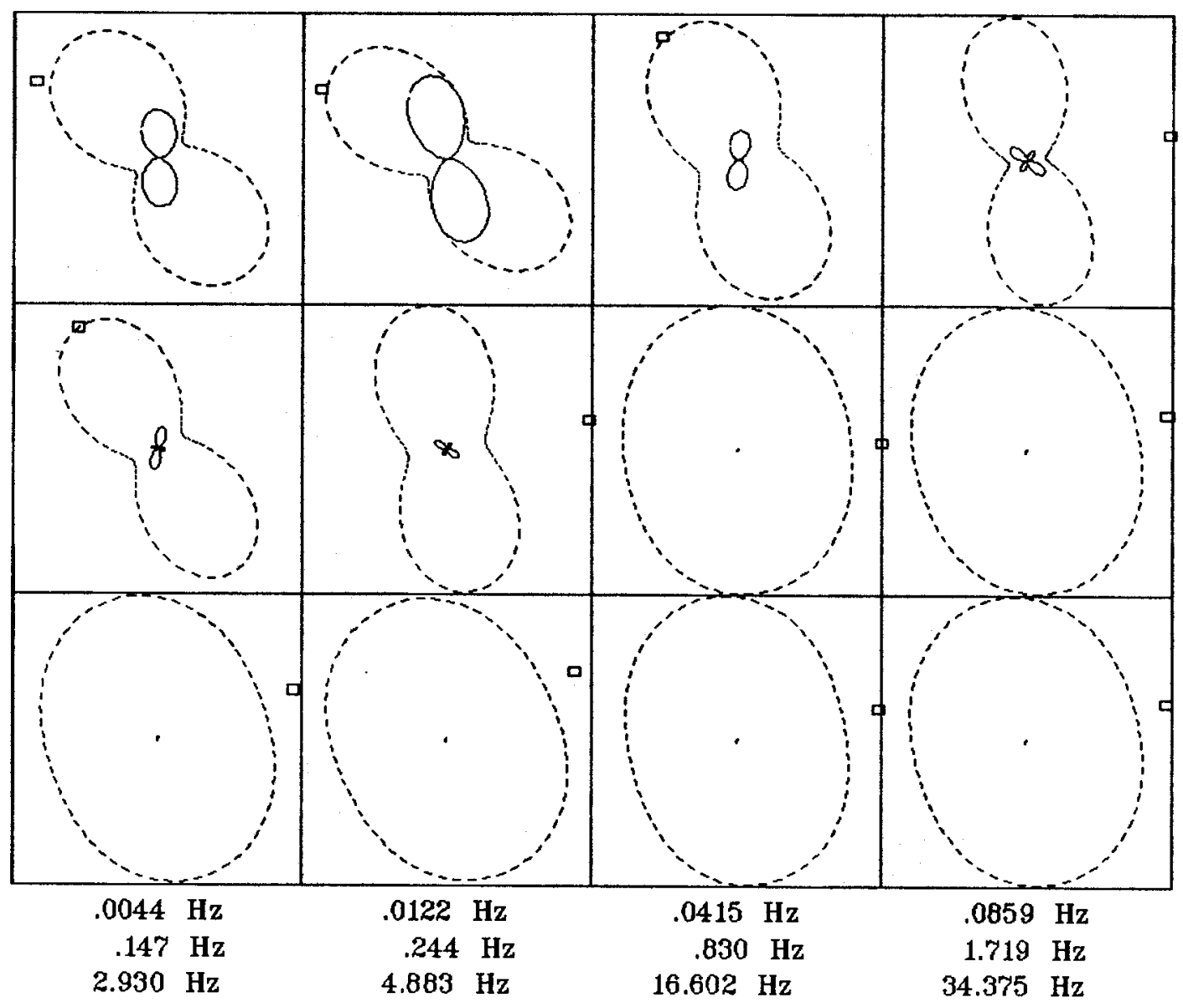

\section{Client:}

Remote: e-field local ref

Acquired: 12:1 Jul 11, 1997

Survey Co:
Rotation:

Filename: nn19.all

Channels: Ch1 Ch2 Ch3 Ch4 Ch5 Ch6 Ch7 Plotted: 08:31 Dec 08, 2000

< EMI - ElectroMagnetic Instruments 
TIPPER MAGNITUDE

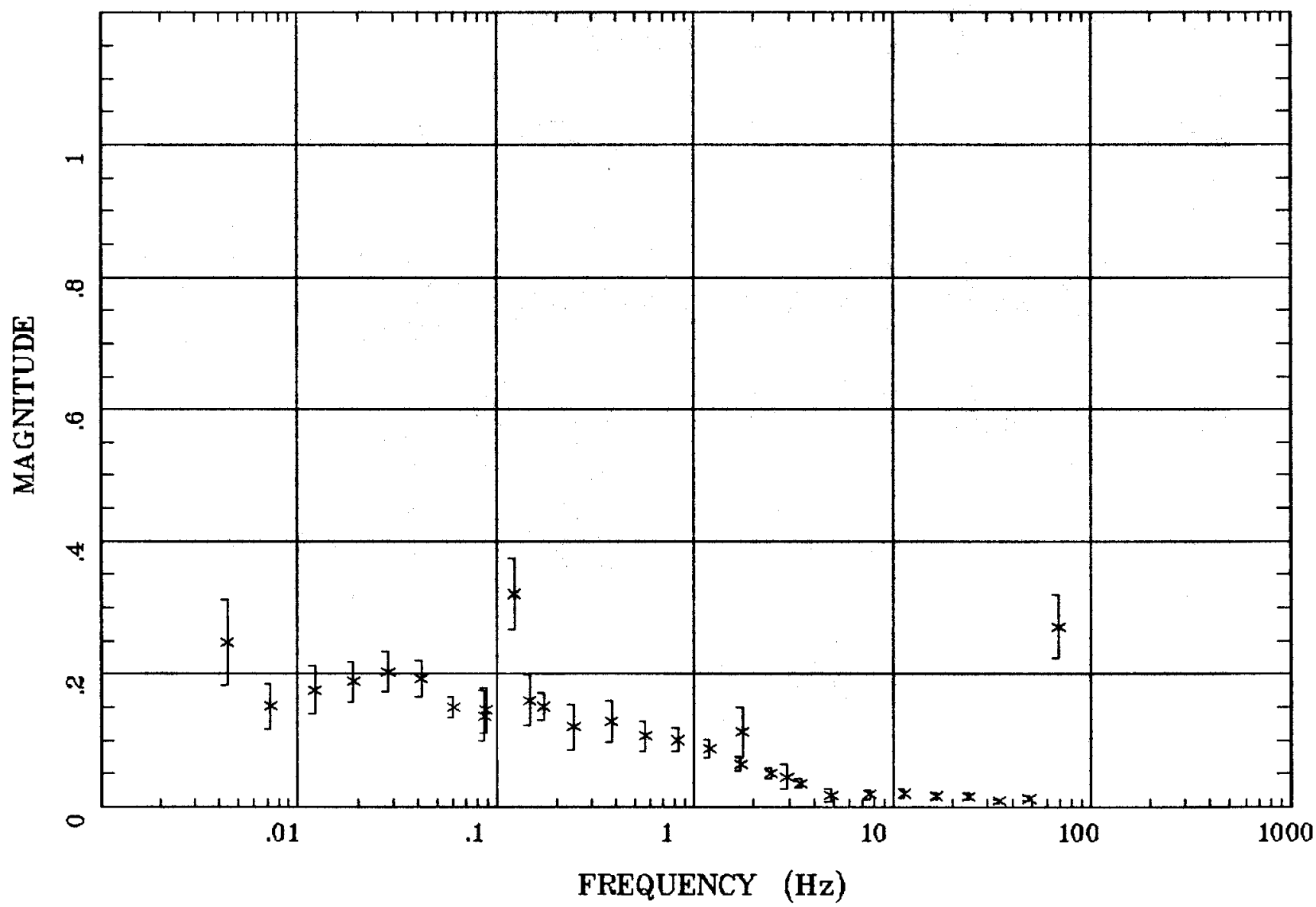

Client:

Remote: e-field local ref Acquired: 12:1 Jul 11, 1997 Survey Co:
Rotation:

Filename: nn19.8ll

Channels: Ch1 Ch2 Ch3 Ch4 Ch5 Ch6 Ch7 Plotted: 08:31 Dec 08, 2000

< EMI - ElectroMagnetic Instruments > 
TIPPER STRIKE

Battle Mtn.

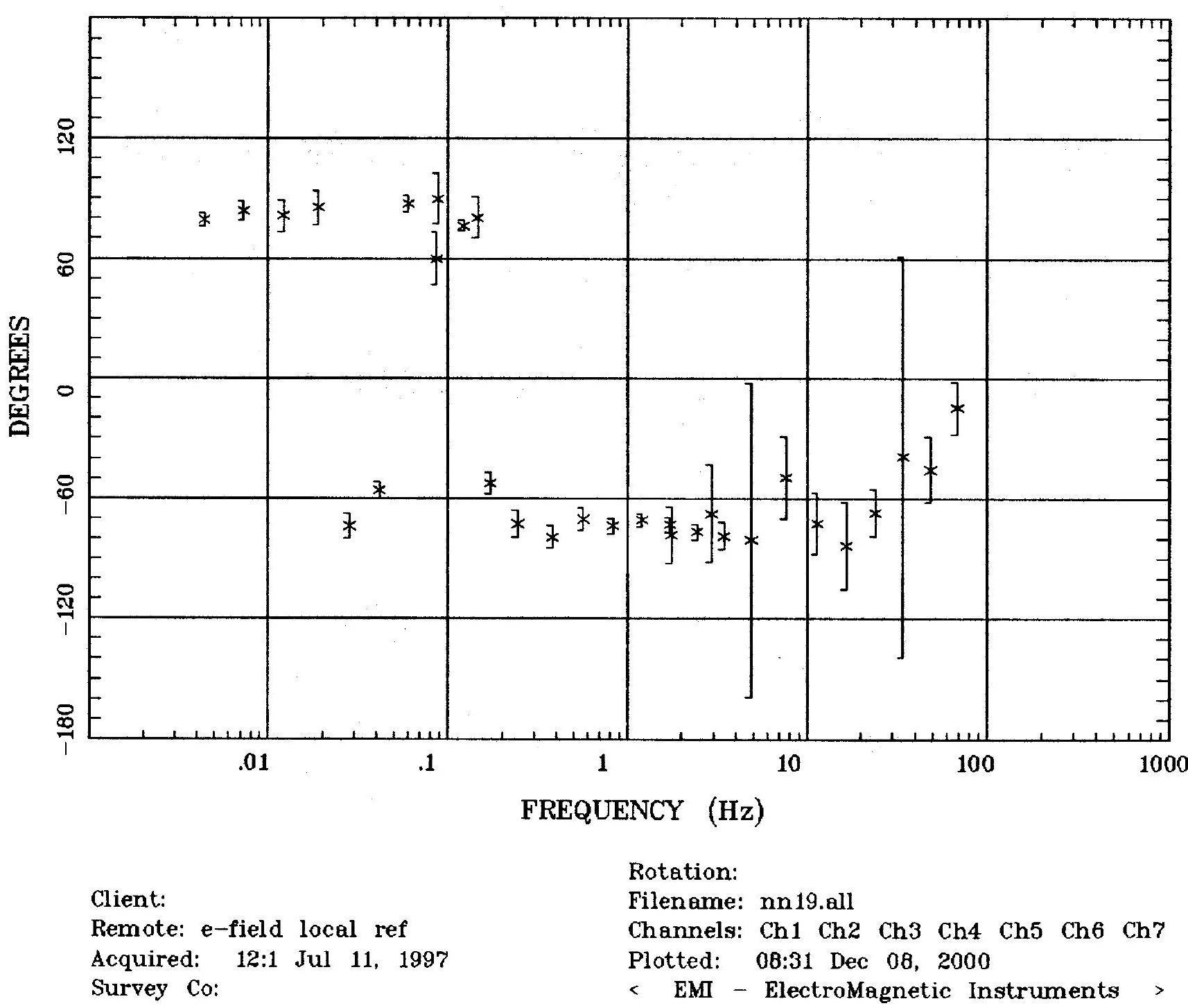


HzHx.x Coh HzHy.o

Battle Mtn.

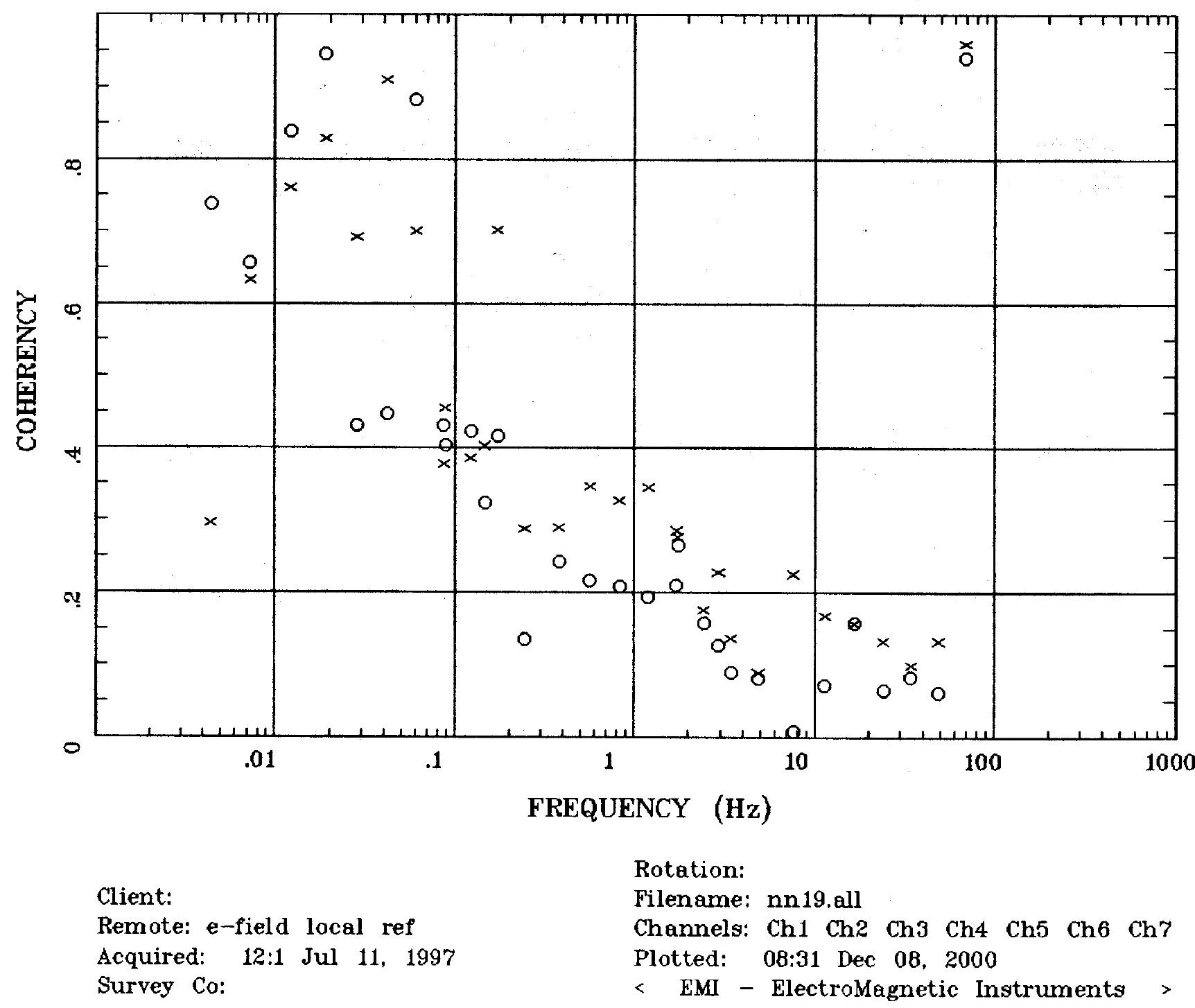




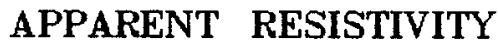

Battle Mtn.

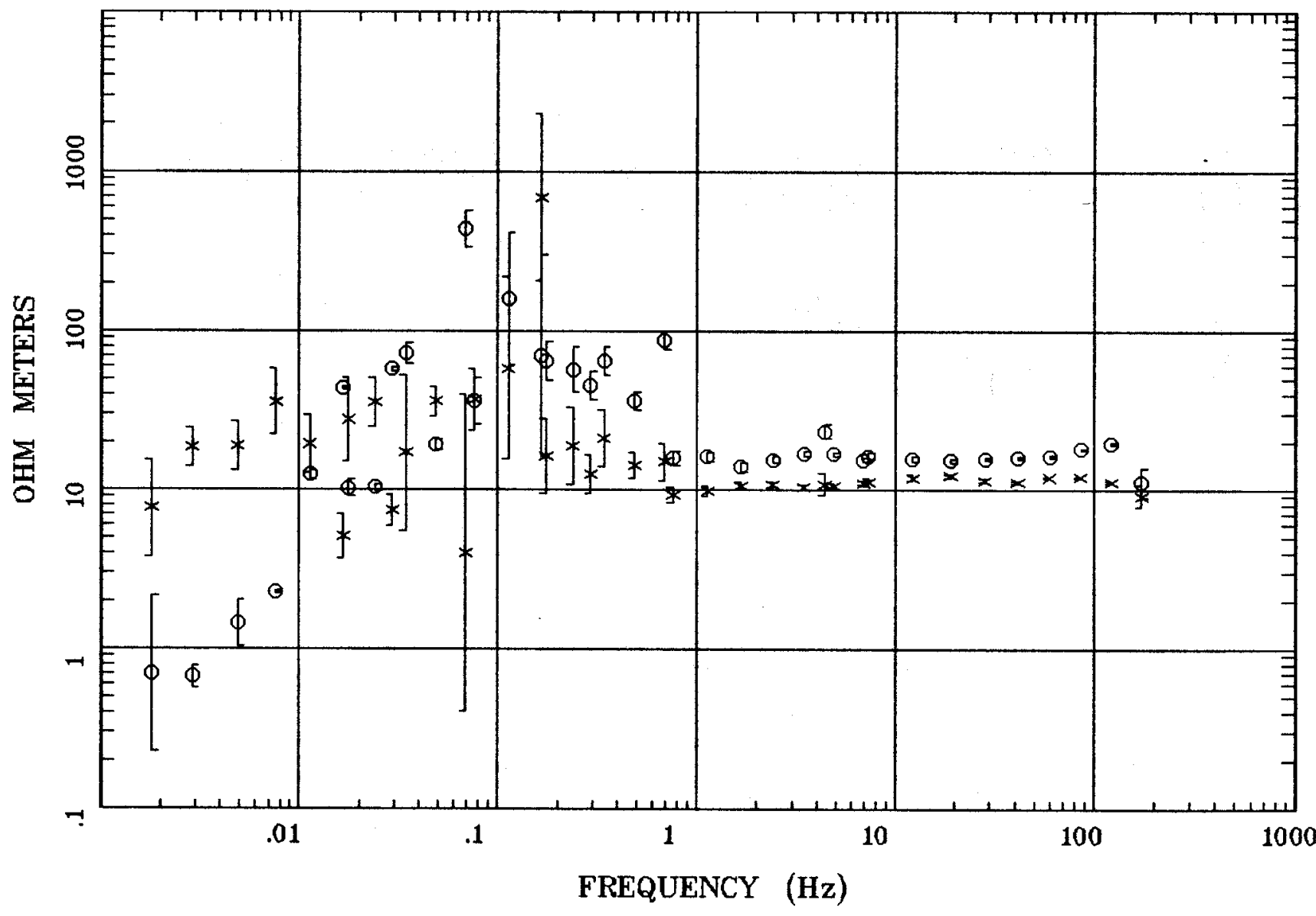

Client:

Remote: E local ref.

Acquired: 15:1 Jul 18, 1997

Survey Co:
Rotation:

Filename: nn20.all

Channels: Ch1 Ch2 Ch3 Ch4 Ch5 Ch6 Ch7

Plotted: 08:32 Dec 08, 2000

$<$ EMI - ElectroMagnetic Instruments 


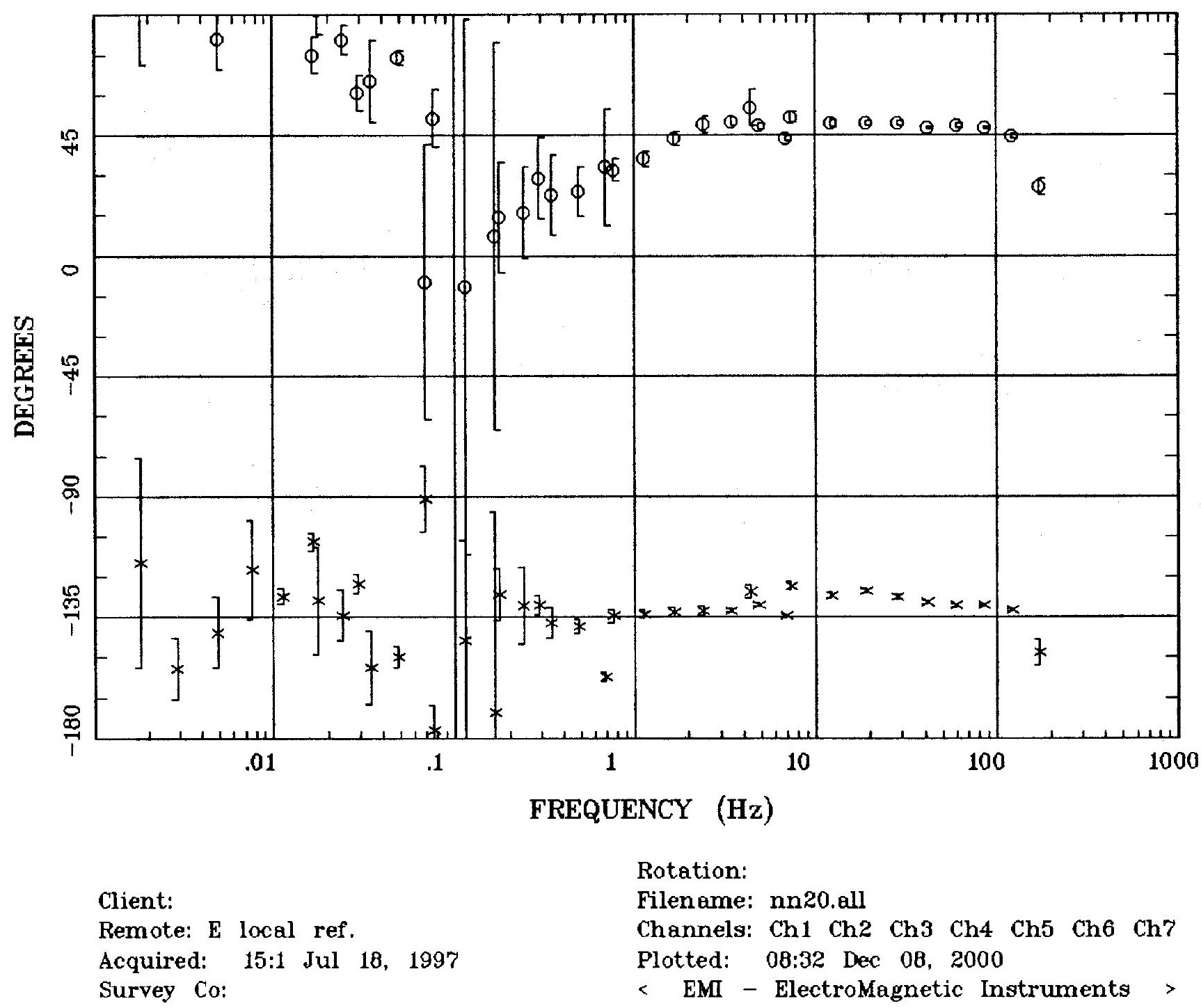


Battle Mtn.

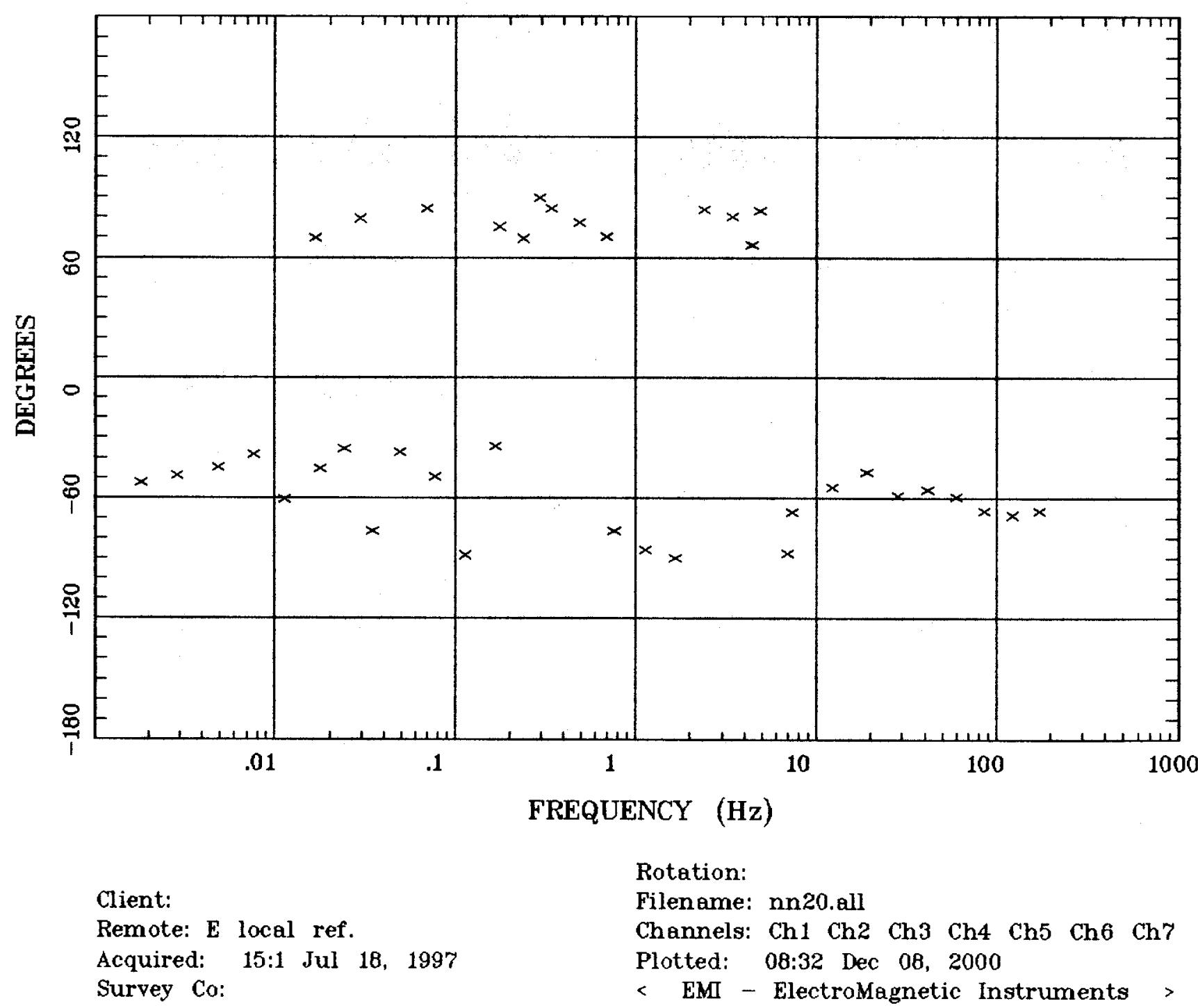


Station 20

IMPEDANCE SKEW

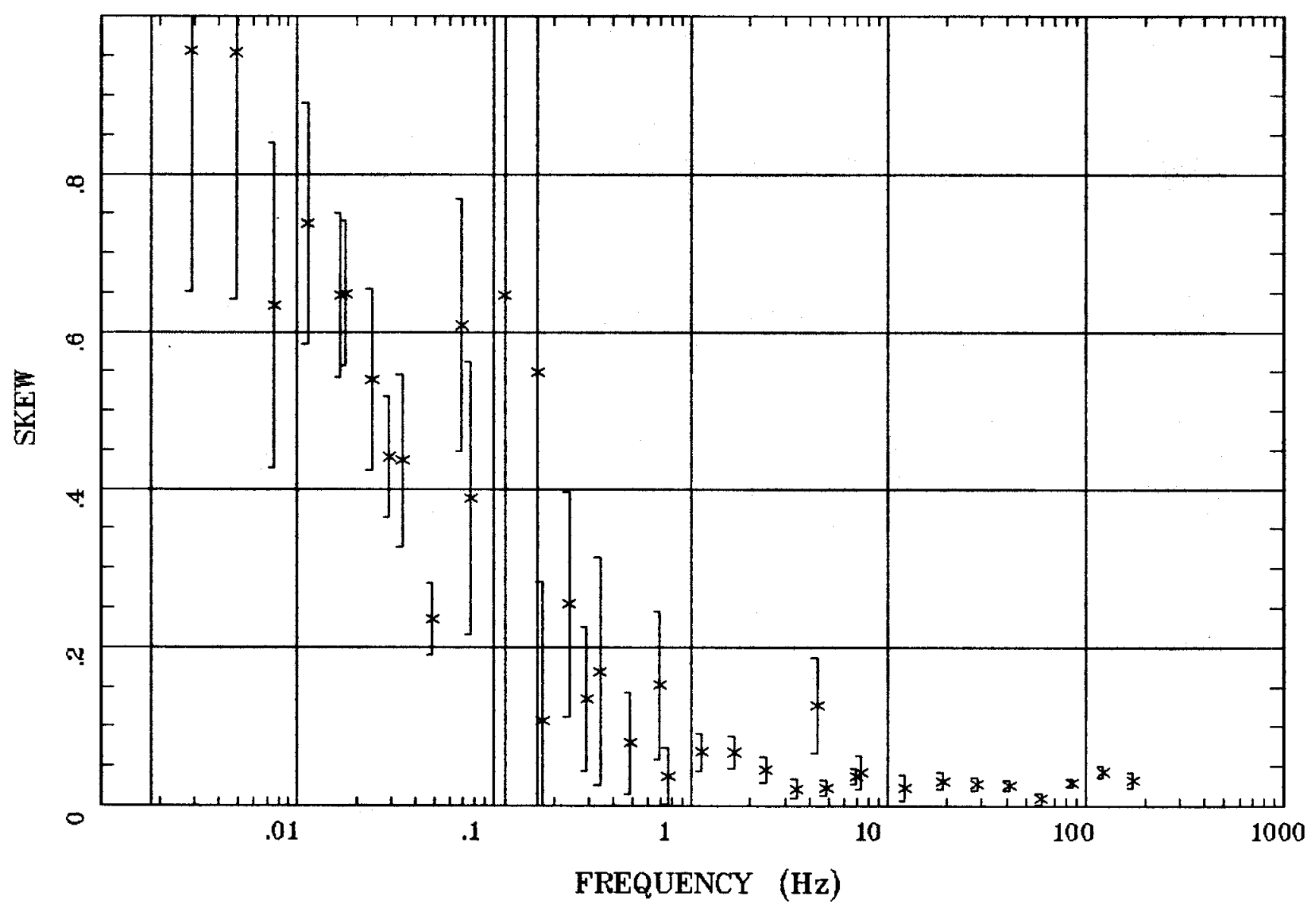

Battle Mtn.

Rotation:

Filename: nnzo.all

Channels: Ch1 Ch2 Ch3 Ch4 Ch5 Ch6 Ch7

Plotted: 08:32 Dec 08, 2000

< EMI - ElectroMagnetic Instruments >

\section{Client:}

Remote: E local ref.

Acquired: 15:1 Jul 18, 1997

Survey Co: 


\section{E MULT Coh.}

Battle Mtn.

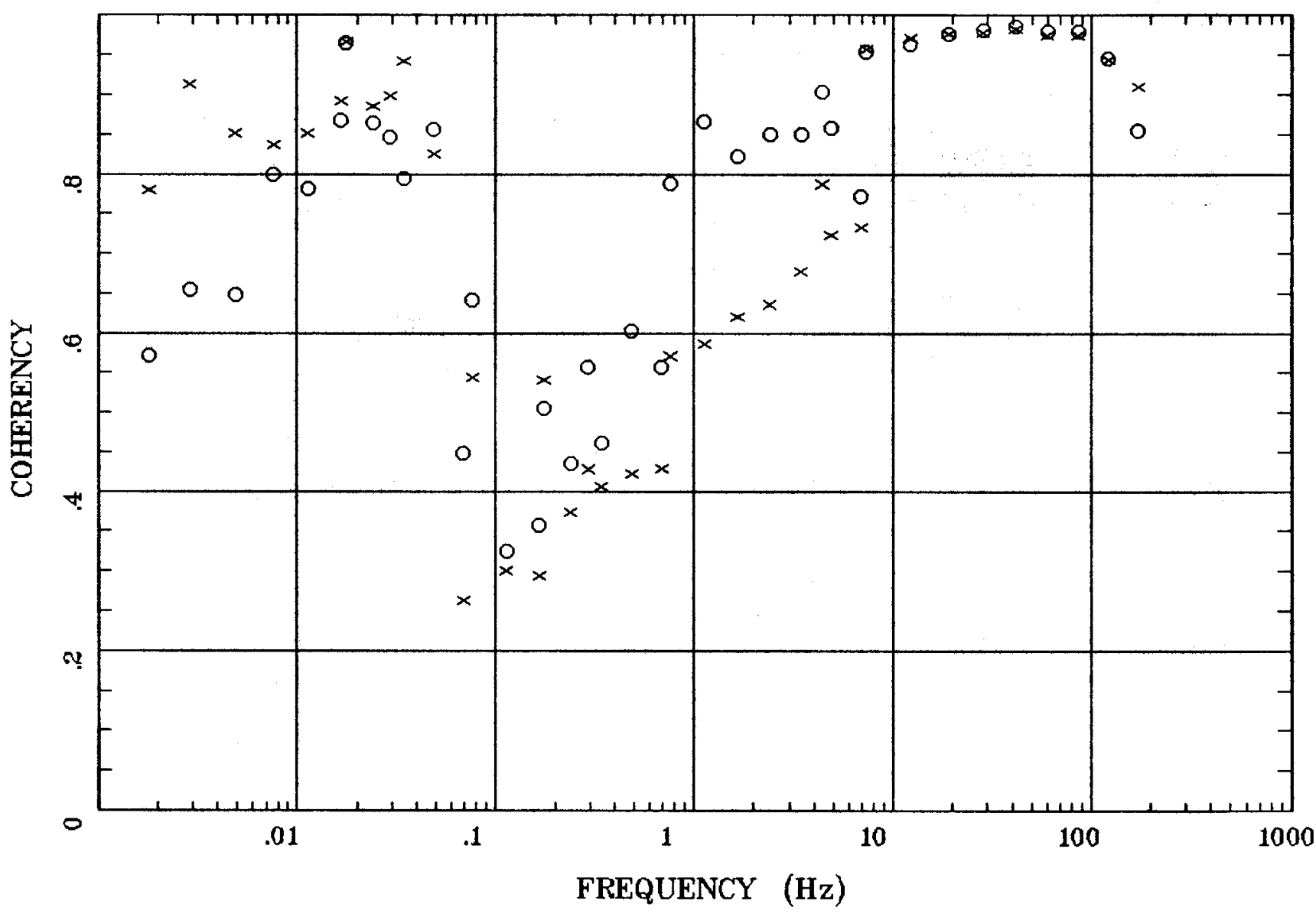

Client:

Remote: E local ref.

Acquired: 15:1 Jul 18, 1997

Survey Co:
Rotation:

Filename: nn20.all

Channels: Ch1 Ch2 Ch3 Ch4 Ch5 Ch6 Ch7 Plotted: 08:32 Dec 08, 2000

$\langle$ EMI - ElectroMagnetic Instruments > 


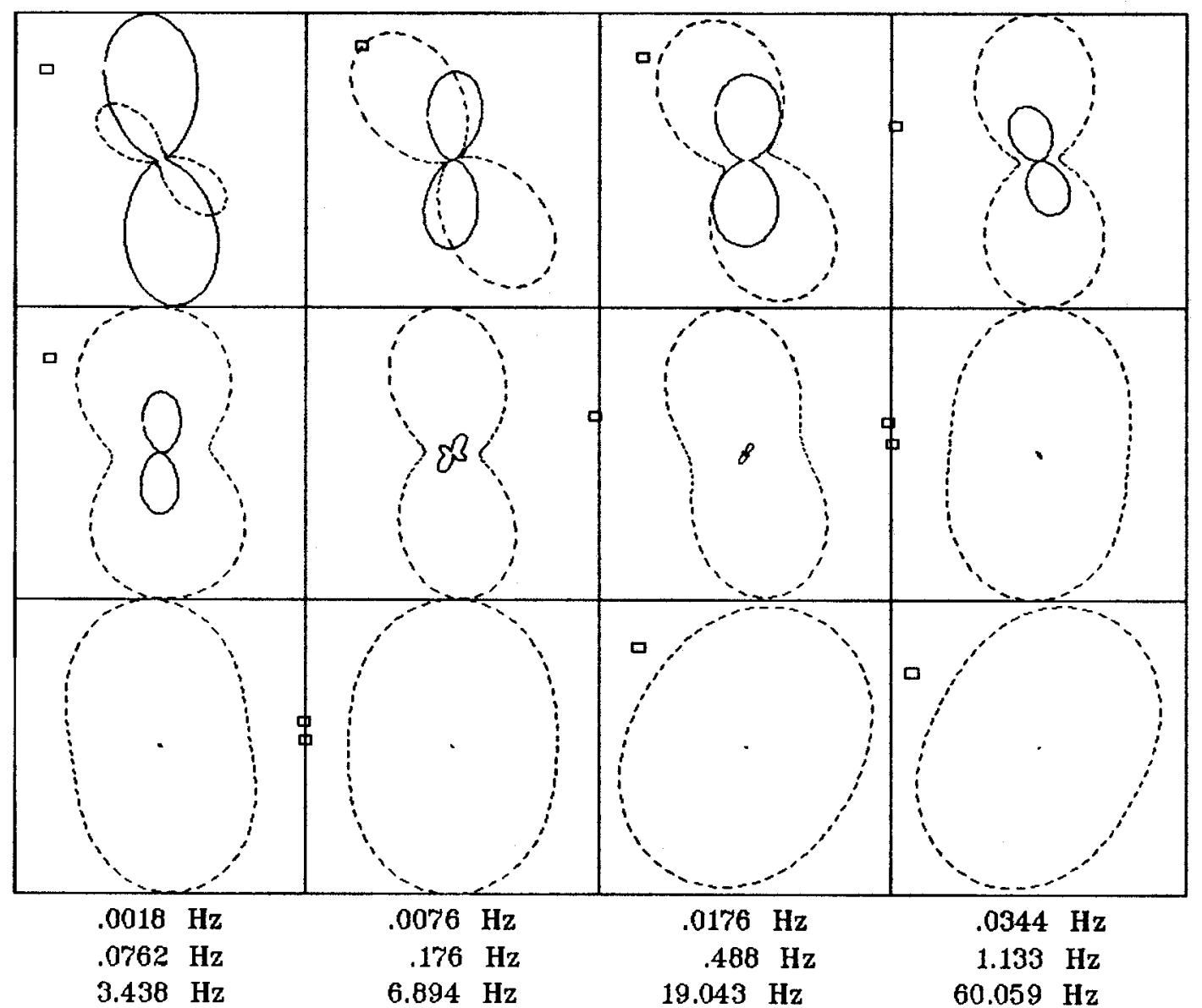

Client:

Remote: $\mathrm{E}$ local ref.

Acquired: 15:1 Jul 18, 1997

Survey Co:
Rotation:

Filename: nn20.all

Channels: Ch1 Ch2 Ch3 Ch4 Ch5 Ch6 Ch7

Plotted: 08:32 Dec 08, 2000

< EMI - ElectroMagnetic Instruments > 


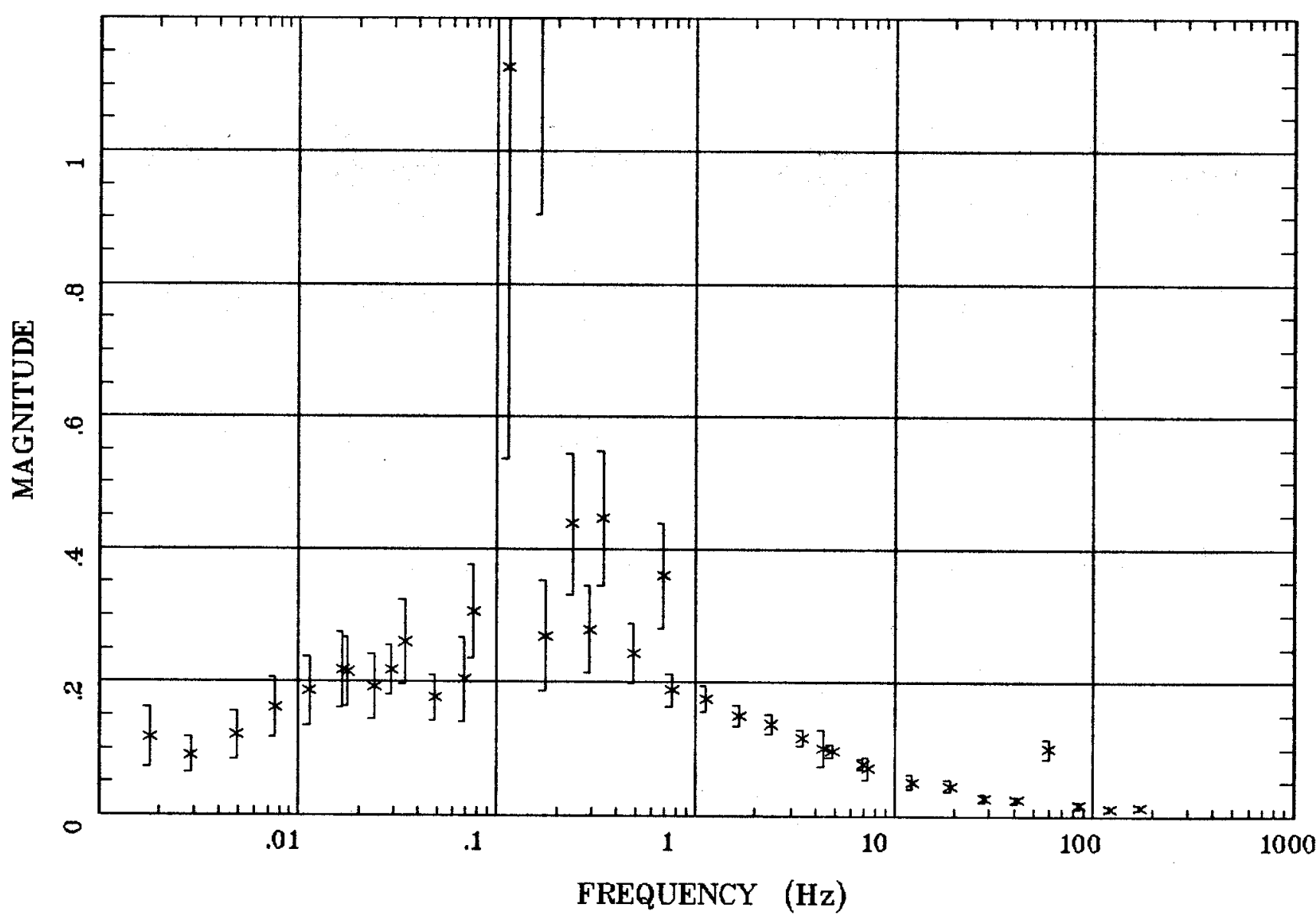

Client:

Remote: E local ref.

Acquired: 15:1 Jul 18, 1997 Survey Co:
Rotation:

Filename: nn20.ell

Channels: Ch1 Ch2 Ch3 Ch4 Ch5 Ch6 Ch7 Plotted: 08:32 Dec 08, 2000

$<$ EMI - ElectroMagnetic Instruments > 
Battle Mtn.

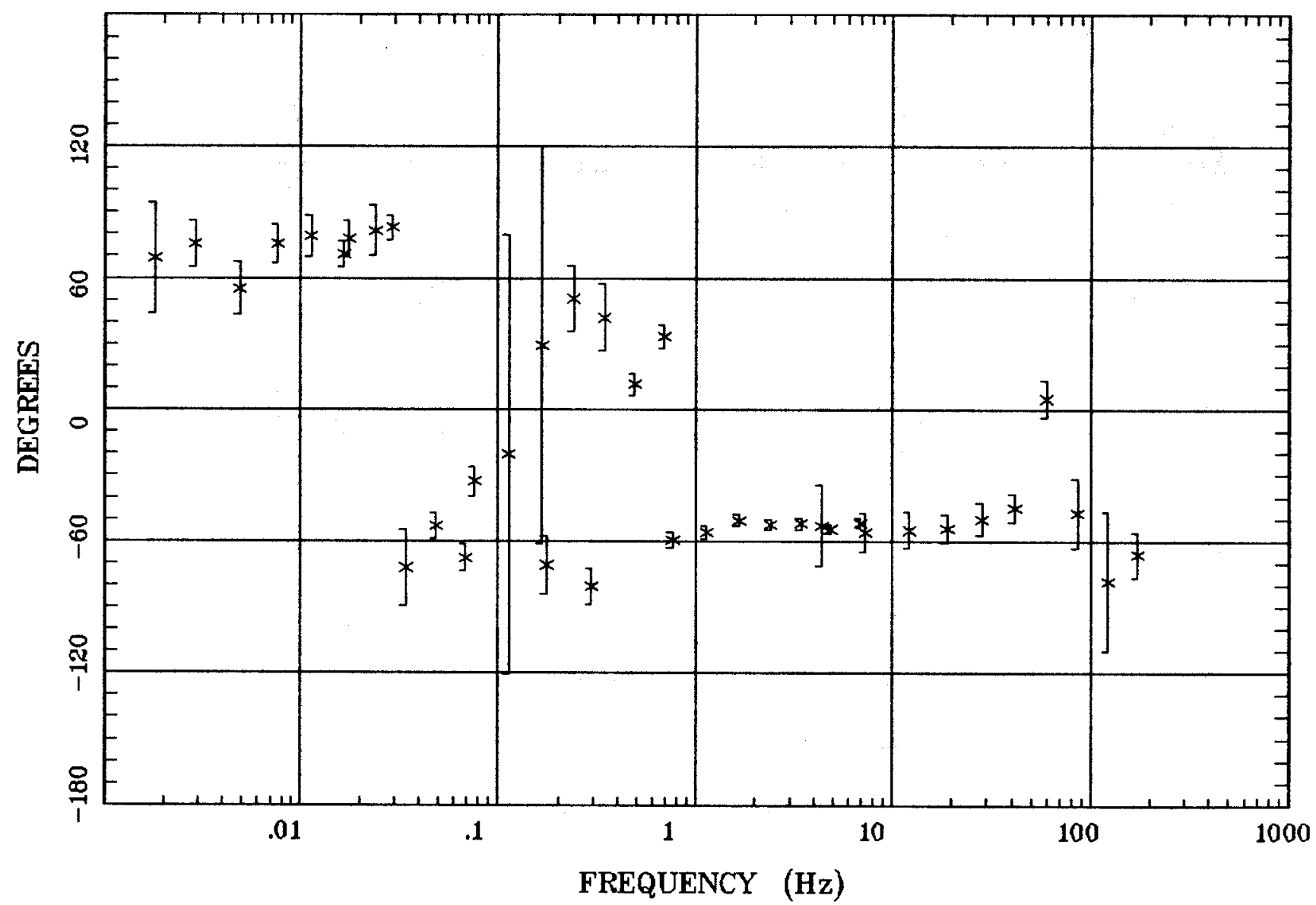

Client:

Remote: E local ref.

Acquired: 15:1 Jul 16, 1997

Survey $\mathrm{Co}$
Rotation:

Filename: nnzo.all

Channels: Ch1 Ch2 Ch3 Ch4 Ch5 Ch6 Ch7

Plotted: 08:32 Dec 08, 2000

$<$ EMI - ElectroMagnetic Instruments 
HzHx.x Coh HzHy.o

Battle Mtn.

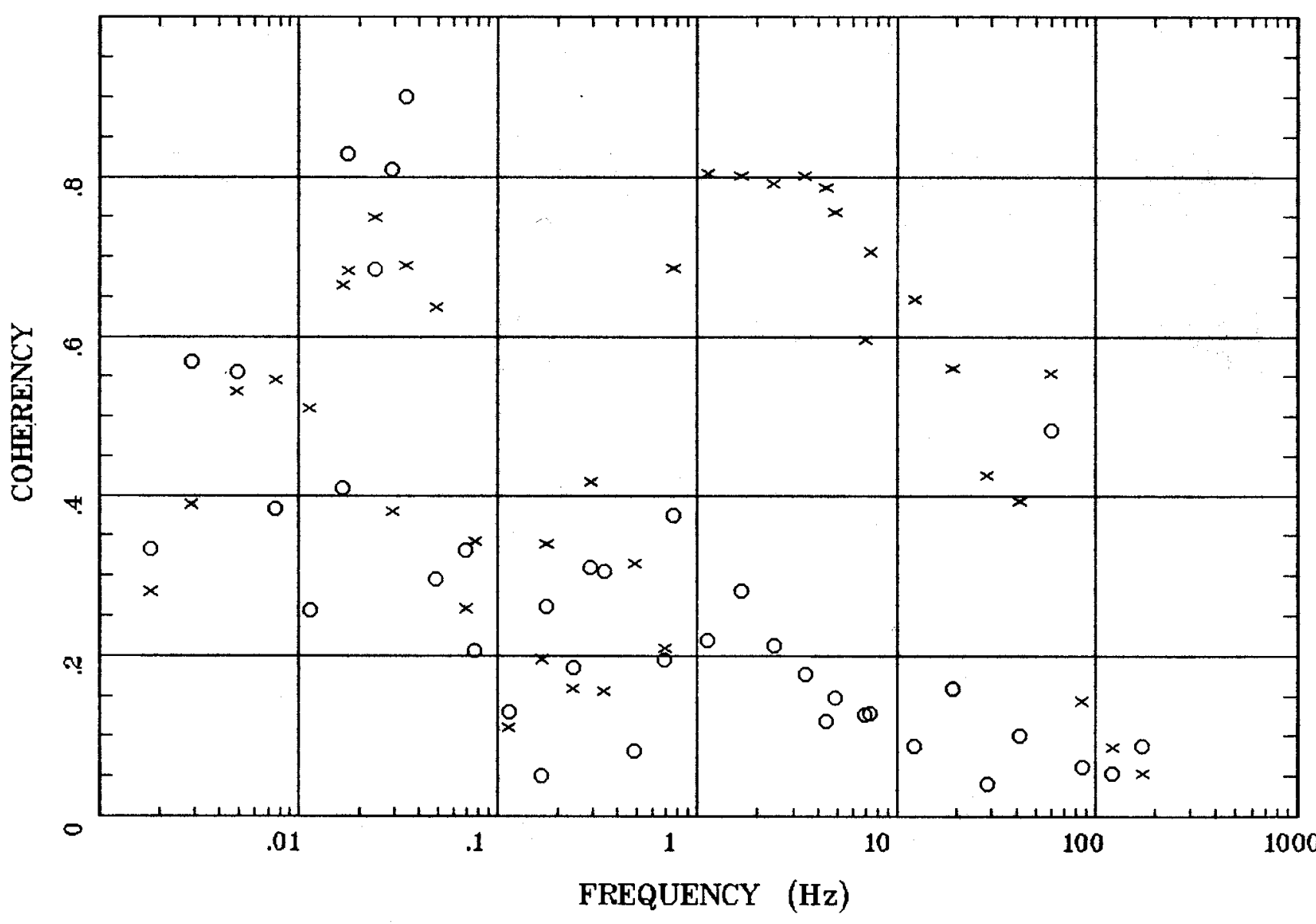

Client:

Remote: E local ref.

Acquired: 15:1 Jul 18, 1997 Survey Co:

Rotation:

Filename: nnzo.all

Channels: Ch1 Ch2 Ch3 Ch4 Ch5 Ch6 Ch7

Plotted: 08:32 Dec 08, 2000

< EMI - ElectroMagnetic Instruments 


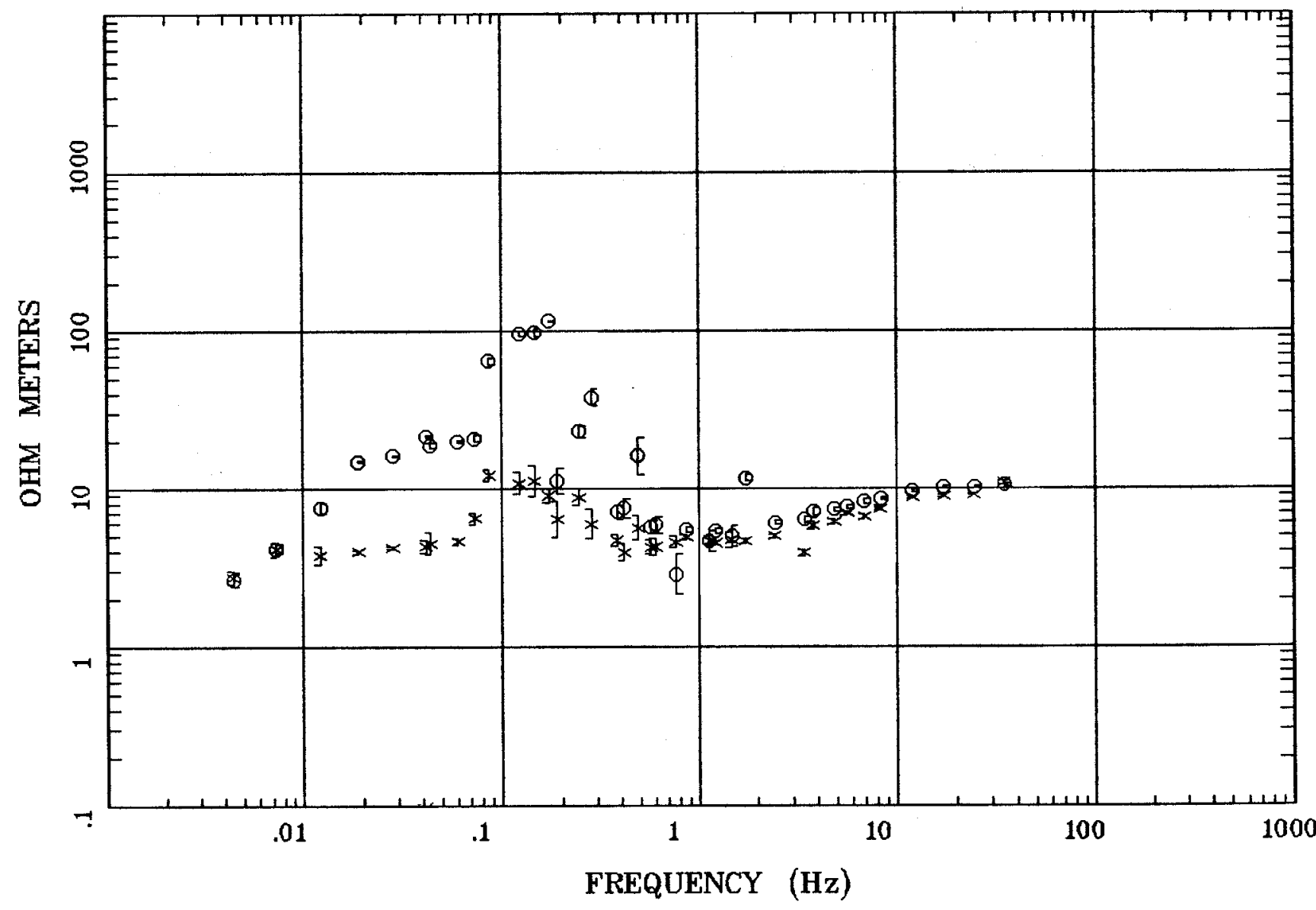

Client:

Remote: e-field local ref Acquired: 19:5 Jul 10, 1997 Survey Co:

\section{Rotation:}

Filename: nnz2e.all Channels: Ch1 Ch2 Ch3 Ch4 Ch5 Ch6 Ch7 Plotted: 08:34 Dec 08, 2000

<EMI - ElectroMagnetic Instruments > 


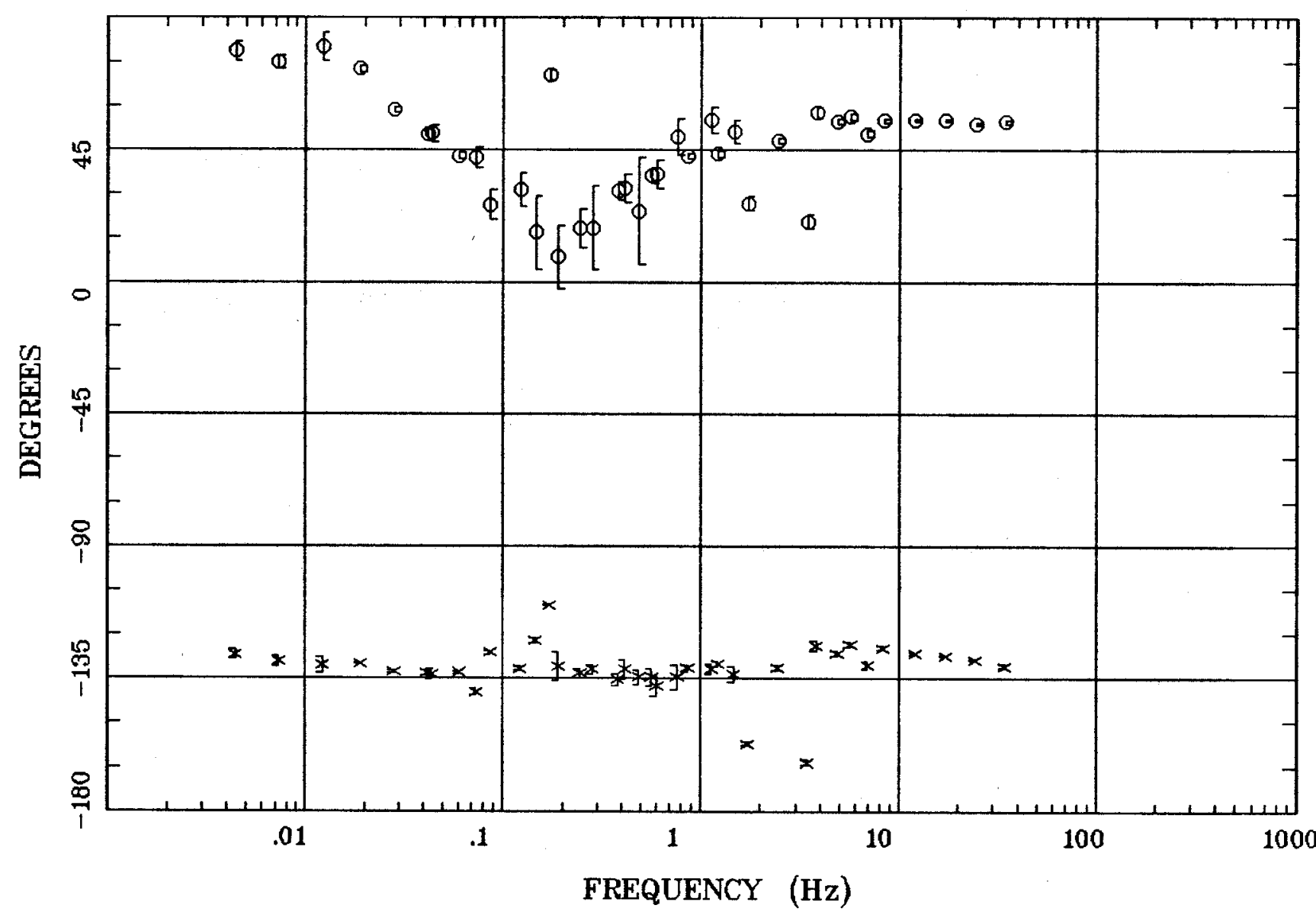

Client:

Remote: e-field local ref Acquired: 19:5 Jul 10, 1997 Survey Co:
Rotation:

Filename: nn22e.all

Channels: Ch1 Ch2 Ch3 Ch4 Ch5 Ch6 Ch7 Plotted: 08:34 Dec 08, 2000

$<$ EMI - ElectroMagnetic Instruments > 
ROTATION ANGLE

Battle Mtn.

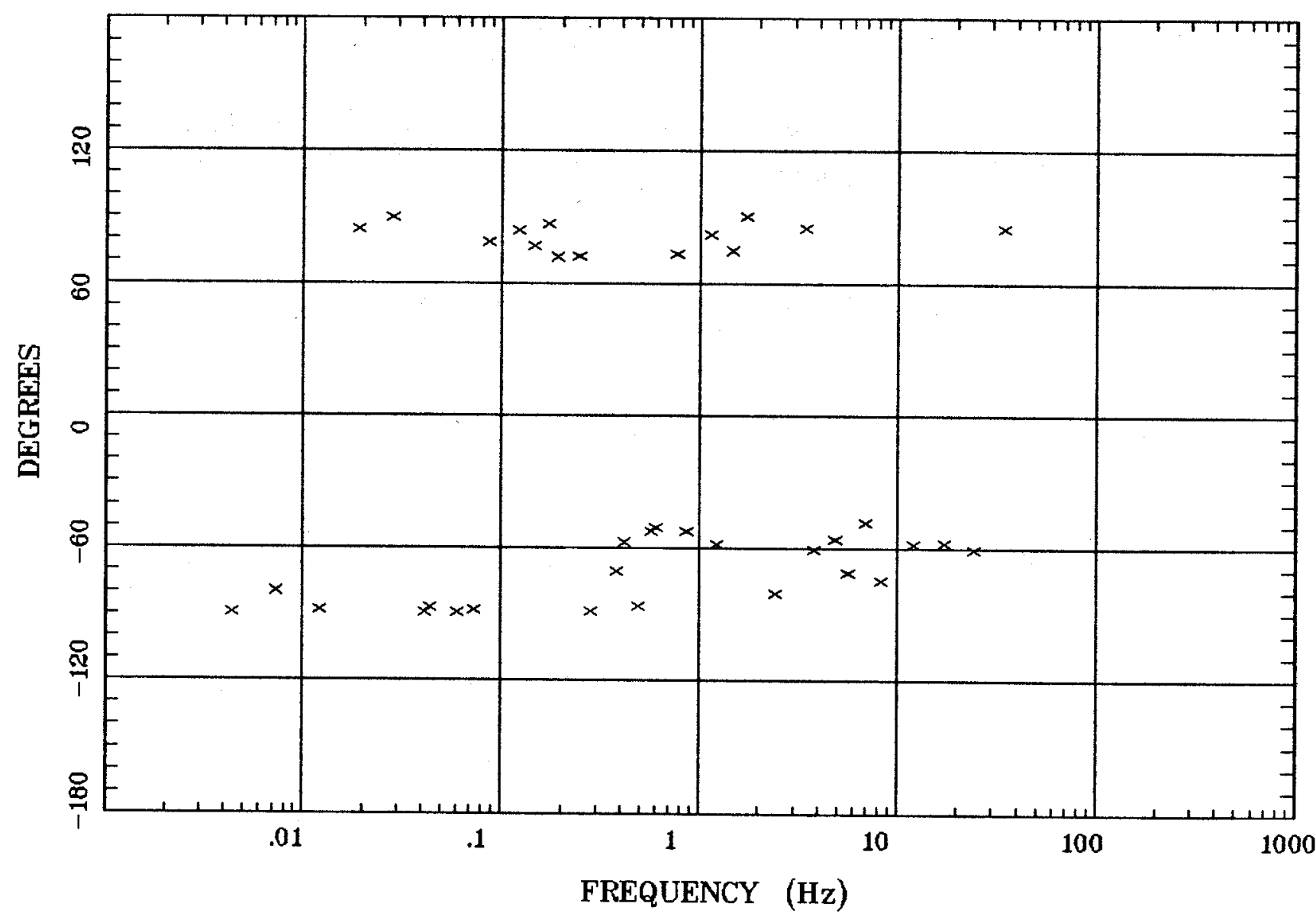

Client:

Remote: e-field local ref Acquired: 19:5 Jul 10, 1997 Survey Co:

\section{Rotation:}

Filename: nn22e.all

Channels: Ch1 Ch2 Ch3 Ch4 Ch5 Ch6 Ch7 Plotted: 08:34 Dec 08, 2000

< EMI - ElectroMagnetic Instruments > 
Battle Mtn.

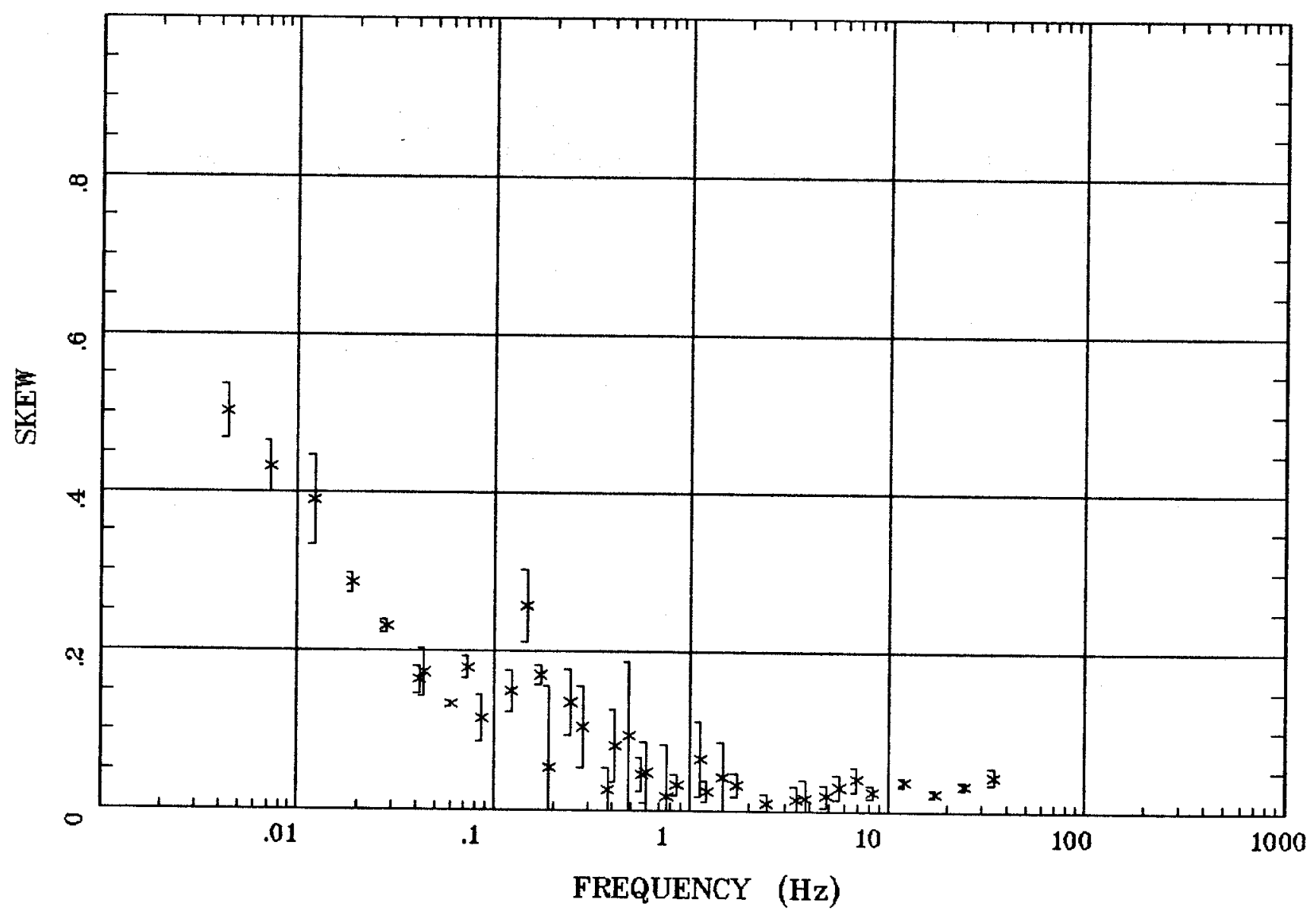

Client:

Rotation:

Remote: e-field local ref Acquired: 19:5 Jul 10, 1997

Filename: nnaze.all

Survey Co:

Channels: Ch1 Ch2 Ch3 Ch4 Ch5 Ch6 Ch7

Plotted: 08:34 Dec 08, 2000

$<$ EMI - ElectroMagnetic Instruments > 


\section{E MULT Coh.}

Battle Mtn.

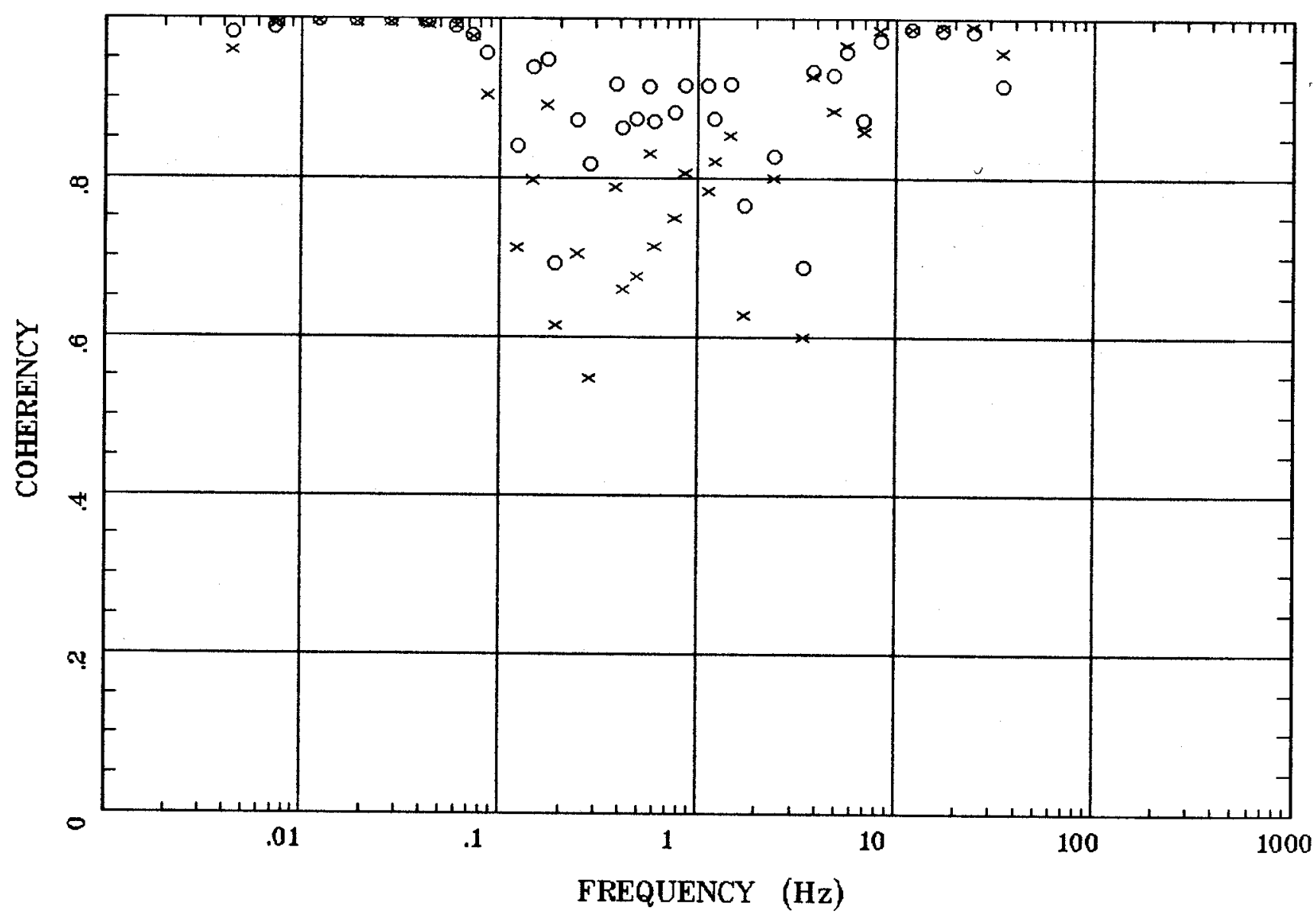

Client:

Remote: e-field local ref Acquired: 19:5 Jul 10, 1997 Survey Co:
Rotation:

Filename: nnaze.all

Channels: Ch1 Ch2 Ch3 Ch4 Ch5 Ch6 Ch7 Plotted: 08:35 Dec 08, 2000

< EMI - ElectroMagnetic Instruments 


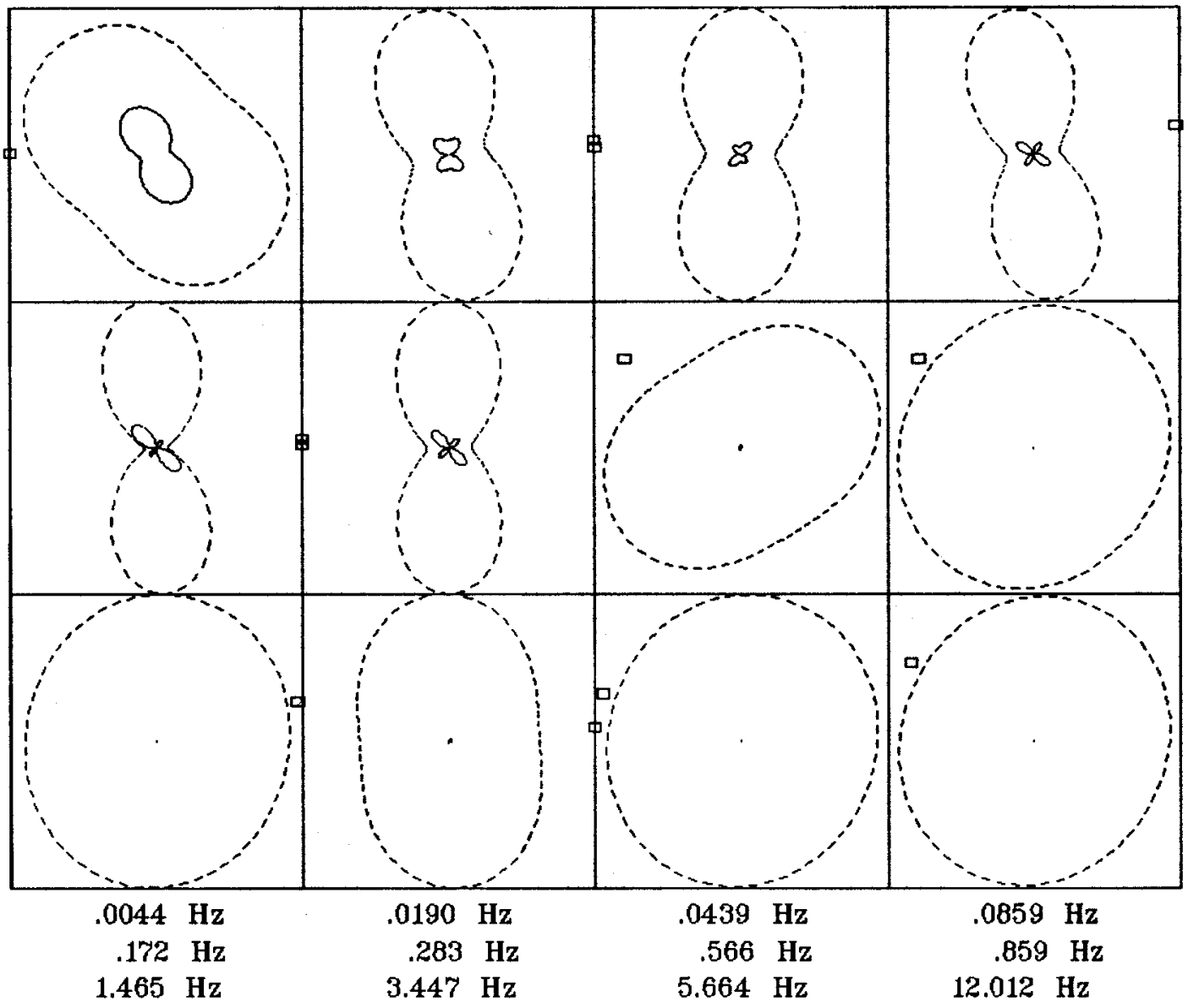

Client:

Remote: e-field local ref

Acquired: 19:5 Jul 10, 1997 Survey Co:
Rotation:

Filename: nnz2e.all

Channels: Ch1 Ch2 Ch3 Ch4 Ch5 Ch6 Ch?

Plotted: 08:35 Dec 08, 2000

< EMI - ElectroMagnetic Instruments 


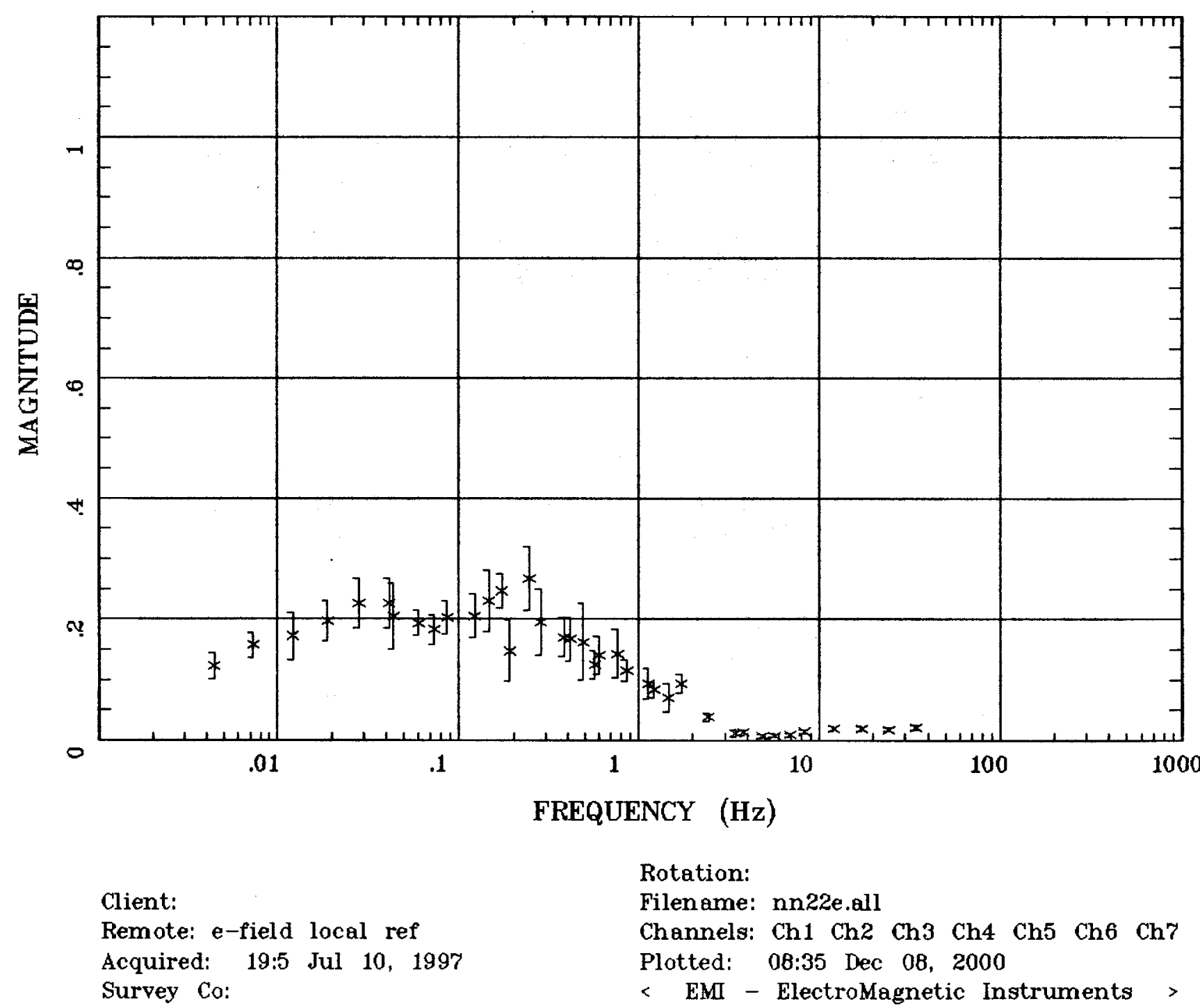


Station 22

TIPPER STRIKE

Battle Mtn.

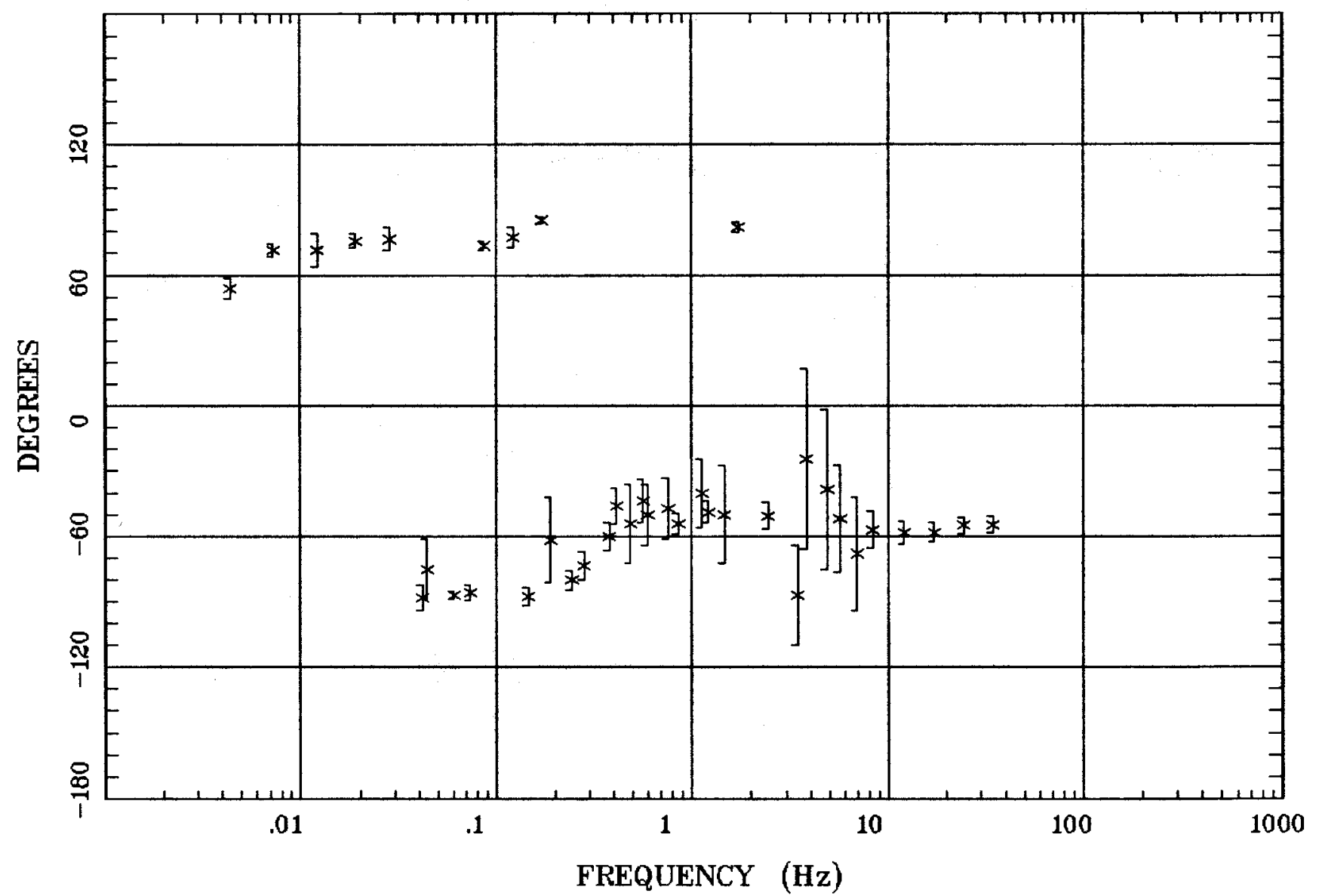

Client:

Rotation:

Remote: e-field local ref

Filename: nnz2e.all

Acquired: 19:5 Jul 10, 1997

Channels: Ch1 Ch2 Ch3 Ch4 Ch5 Ch6 Ch7

Plotted: 08:35 Dec 08, 2000

Survey Co:

< EMI - ElectroMagnetic Instruments 


\section{HzHx.x Coh HzHy.o}

Battle Mtn.

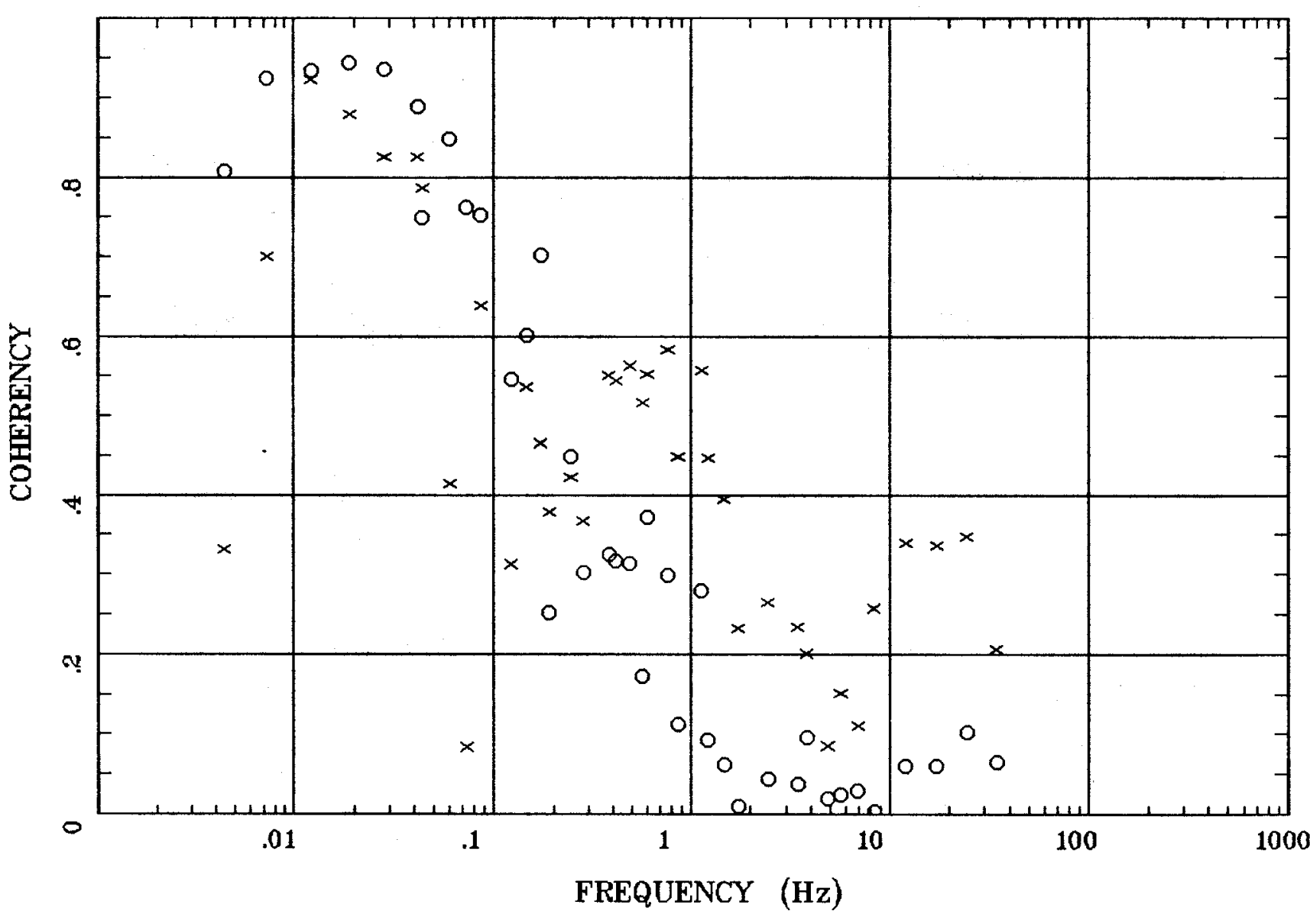

Client:

Remote: e-field local ref Acquired: 19:5 Jul 10, 1997 Survey Co:
Rotation:

Filename: nn22e.all

Channels: Ch1 Ch2 Ch3 Ch4 Ch5 Ch6 Ch7 Plotted: 08:35 Dec 08, 2000

< EMI - ElectroMagnetic Instruments 


\section{APPARENT RESISTIVITY}

Battle Mtn.

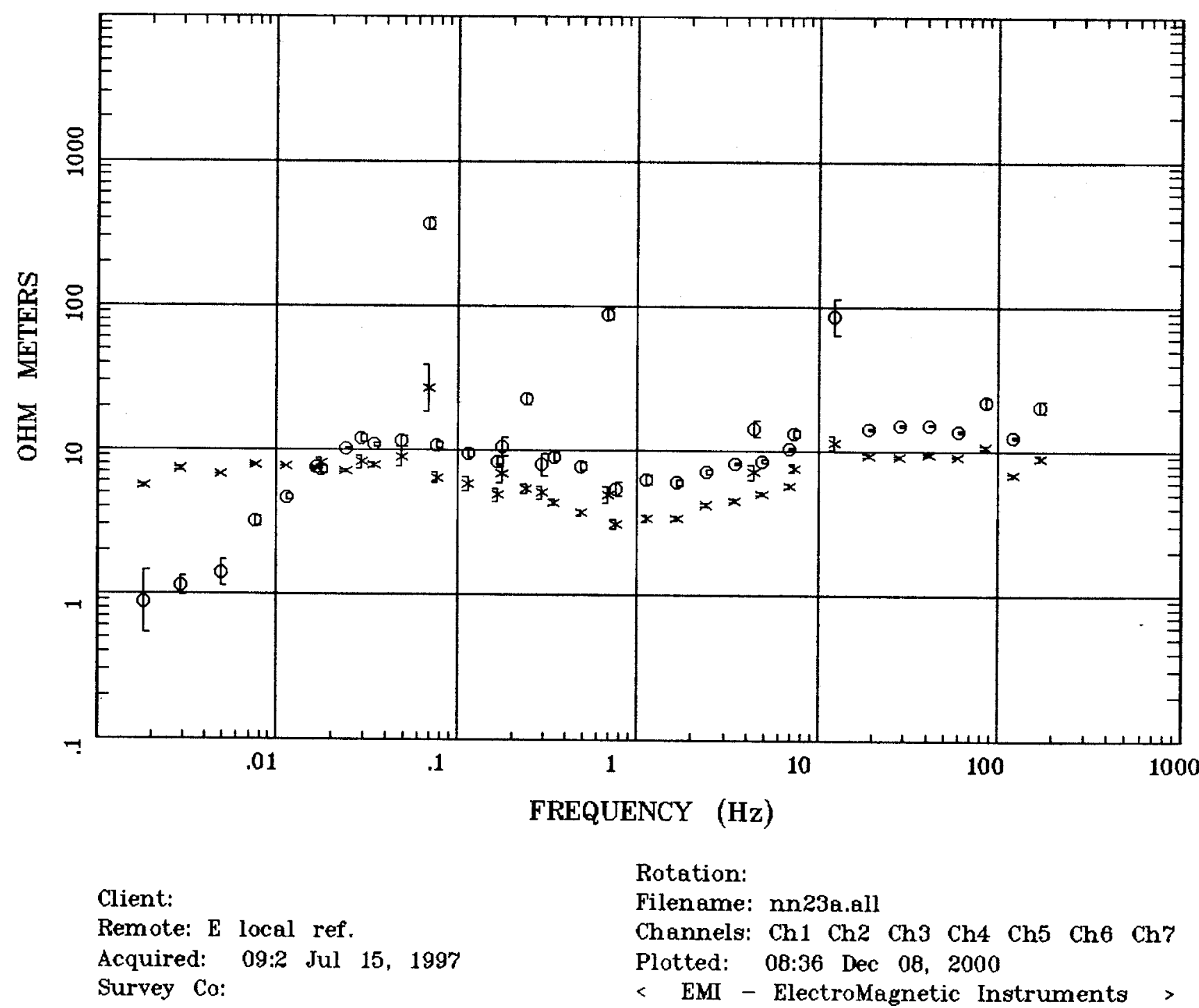


Station 23

IMPEDANCE PHASE

Battle Mtn.

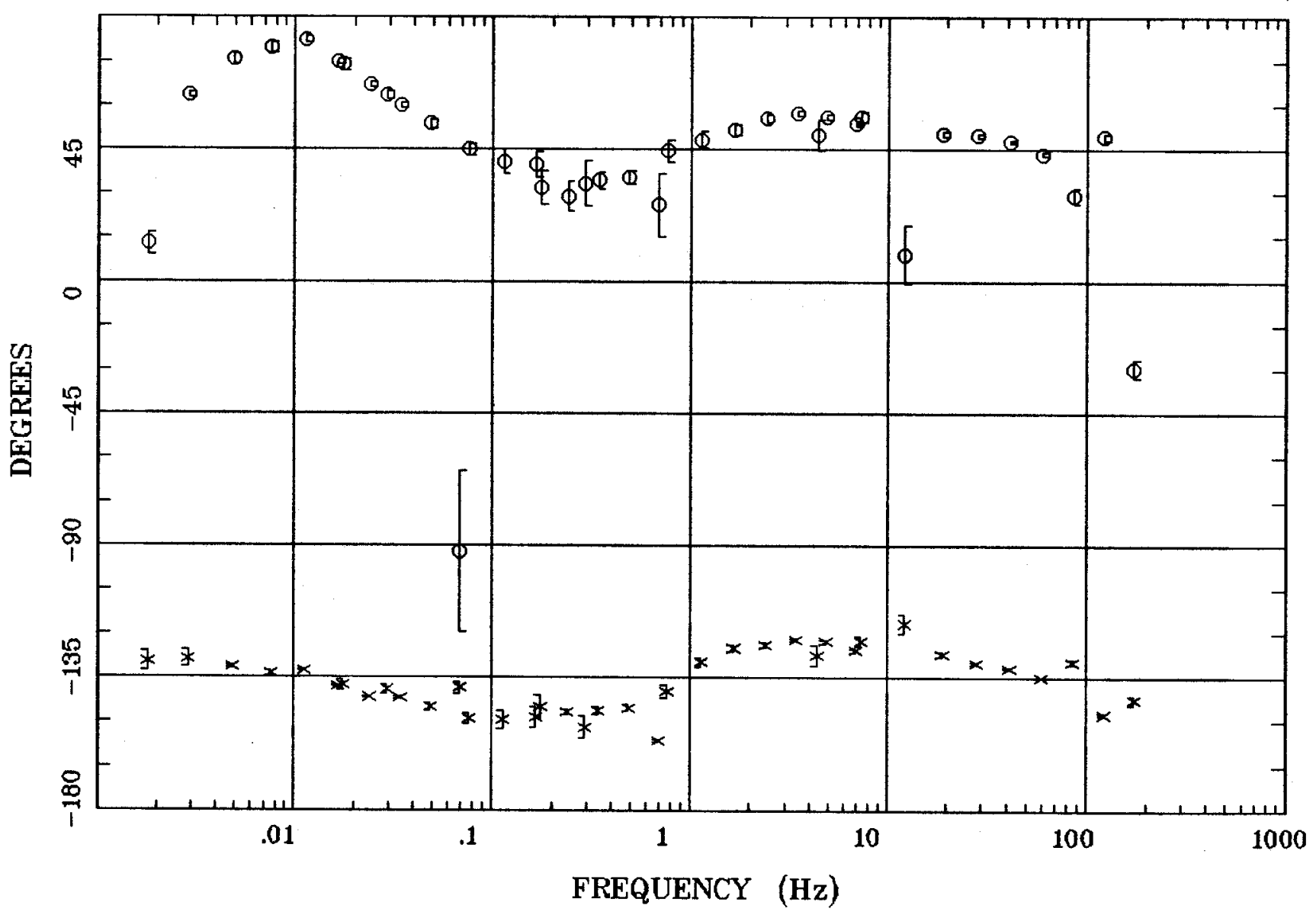

Client:

Remote: E local ref.

Acquired: 09:2 Jul 15, 1997

Survey Co:
Rotation:

Filename: nn23a.all

Channels: Ch1 Ch2 Ch3 Ch4 Ch5 Ch6 Ch7

Plotted: 08:36 Dec 08, 2000

< EMI - ElectroMagnetic Instruments 
ROTATION ANGLE

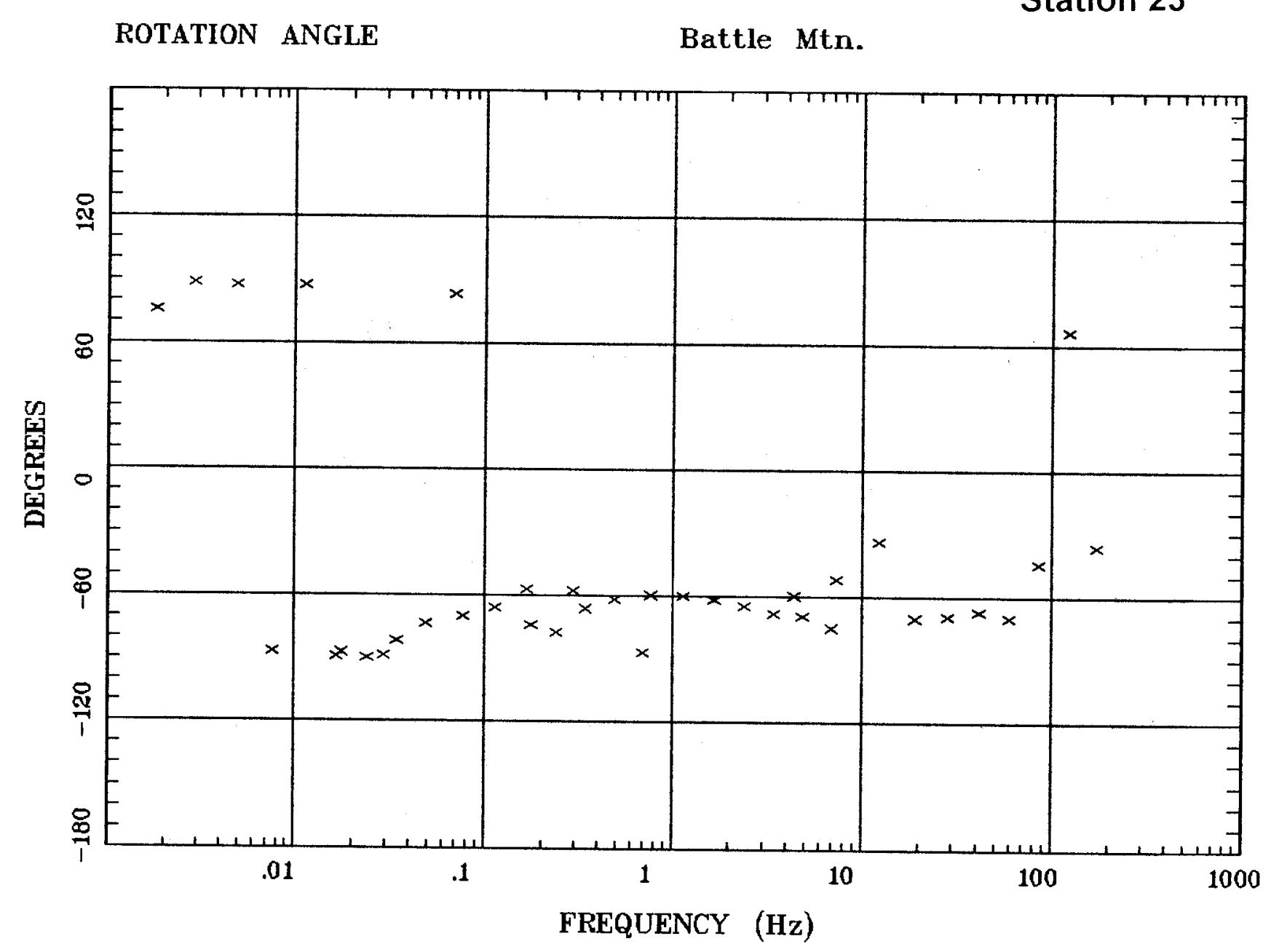

Client:

Remote: E local ref.

Acquired: 09:2 Jul 15, 1997

Survey Co:

Battle Mtn.

Station 23

Rotation:

Filename: nn23a.all

Channels: Ch1 Ch2 Ch3 ch4 Ch5 Ch6 Ch7

Plotted: 08:36 Dec 08, 2000

< EMI - ElectroMagnetic Instruments > 
IMPEDANCE SKEW

Battle Mtn.

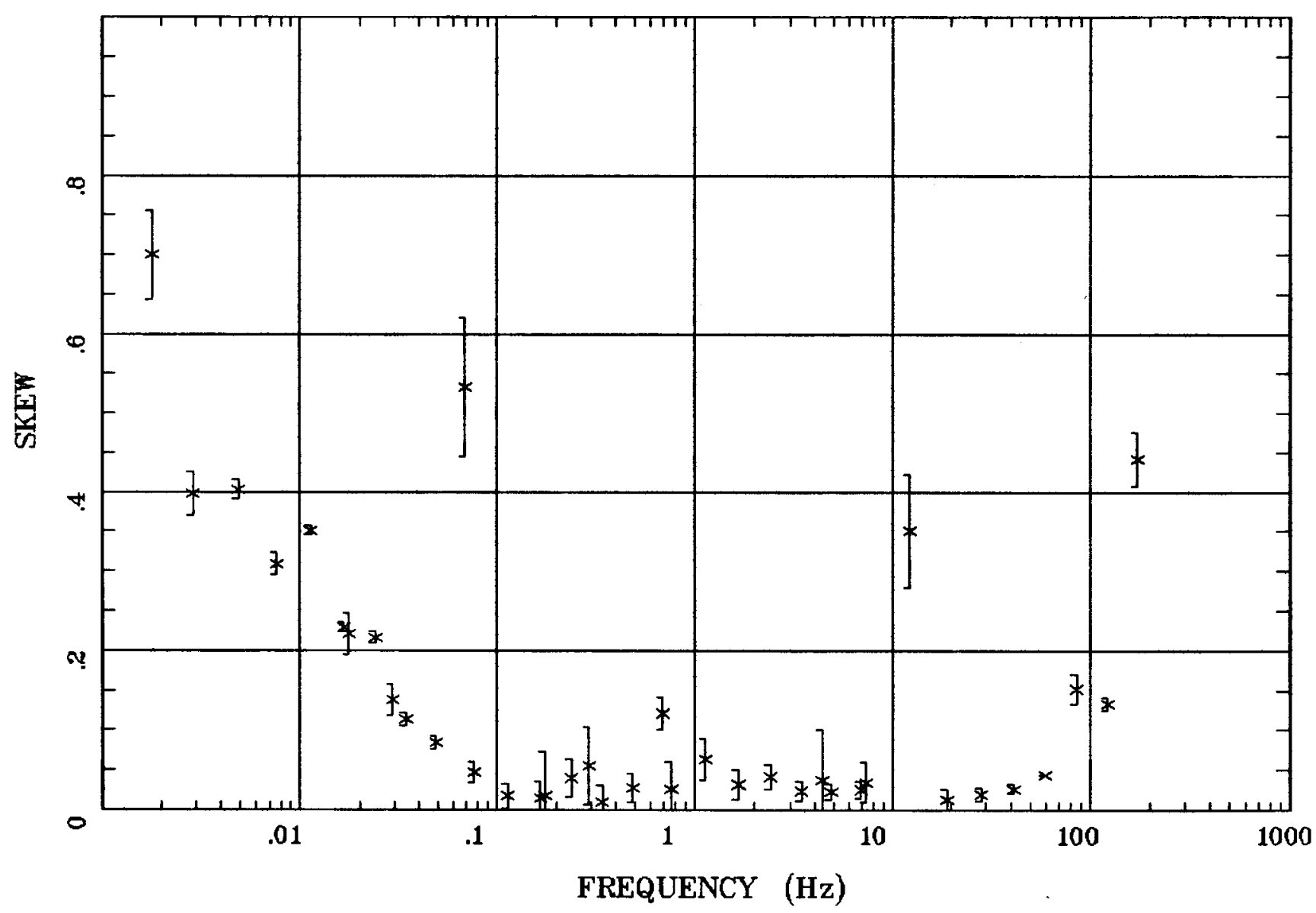

Client:

Remote: E local ref.

Acquired: 09:2 Jul 15, 1997 Survey Co:
Rotation:

Filename: nn23a.all

Channels: Ch1 Ch2 Ch3 Ch4 Ch5 Ch6 Ch7 Plotted: 08:36 Dec 08, 2000

< EMI - ElectroMagnetic Instruments 


\section{Station 23}

\section{E MULT Coh.}

\section{Battle Mtn.}

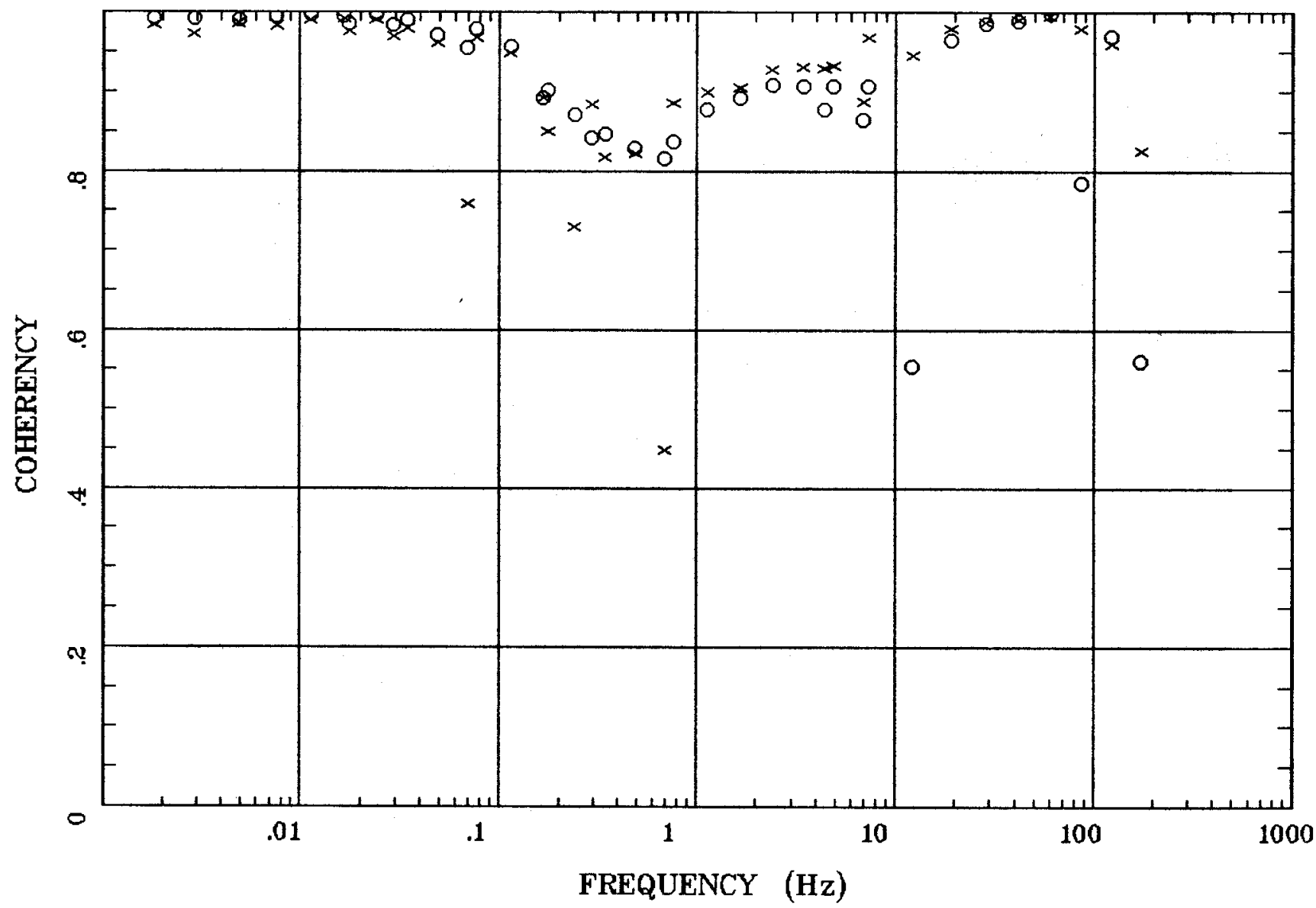

\section{Client:}

Remote: E local ref.

Acquired: 09:2 Jul 15, 1997

Survey Ca:
Rotation:

Filename: nn23a.all

Channels: Ch1 Ch2 Ch3 Ch4 Ch5 Ch6 Ch7

Plotted: 08:36 Dec 08, 2000

$<$ EMI - ElectroMagnetic Instruments 
Station 23

POLAR PLOTS

Battle Mtn.

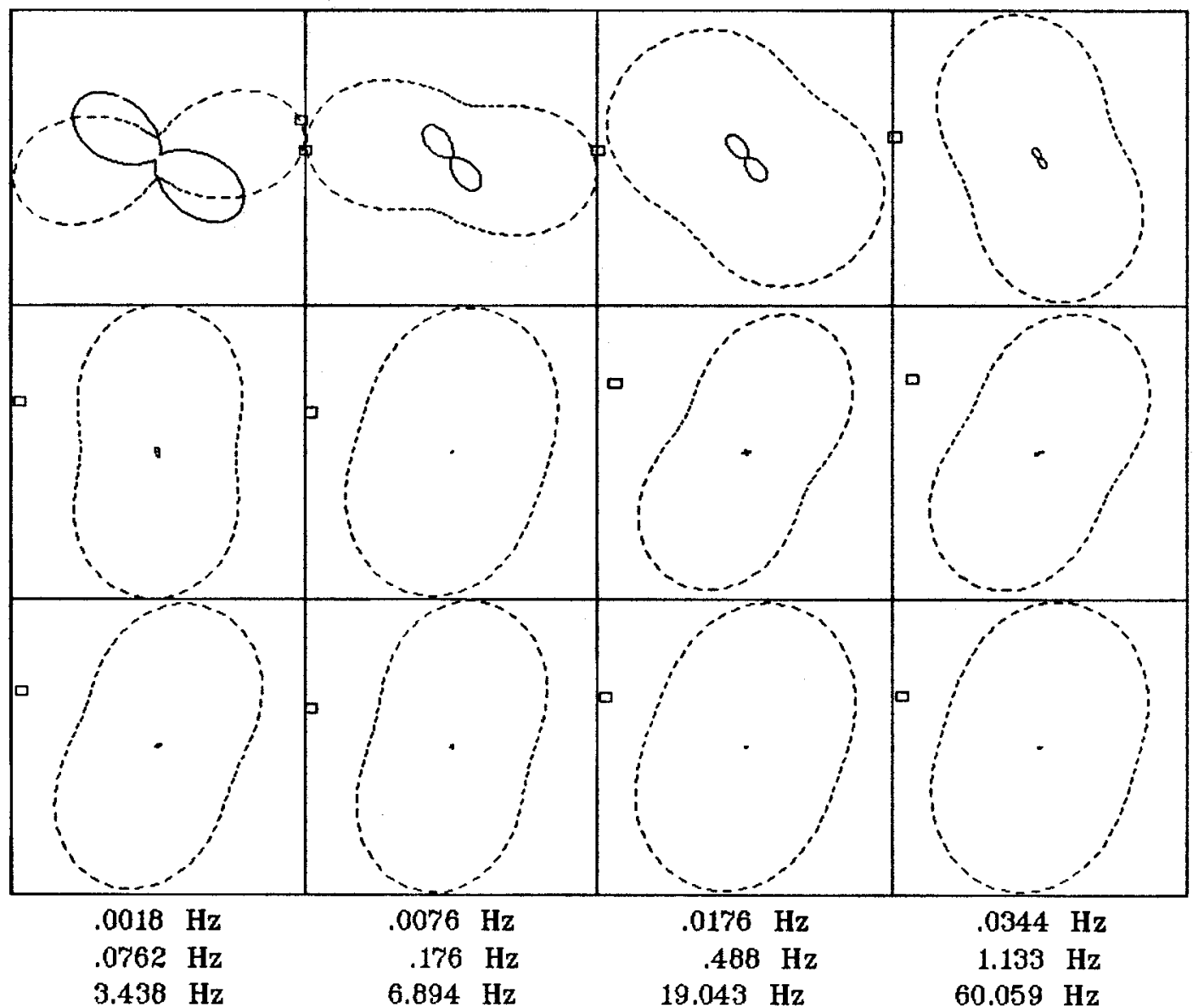

Client:

Remote: E local ref.

Acquired: 09:2 Jul 15, 1997

Survey Co:
Rotation:

Filename: nn23a.all

Channels: Ch1 Ch2 Ch3 Ch4 Ch5 Ch6 Chr

Plotted: 08:36 Dec 08, 2000

< EMI - ElectroMagnetic Instruments > 


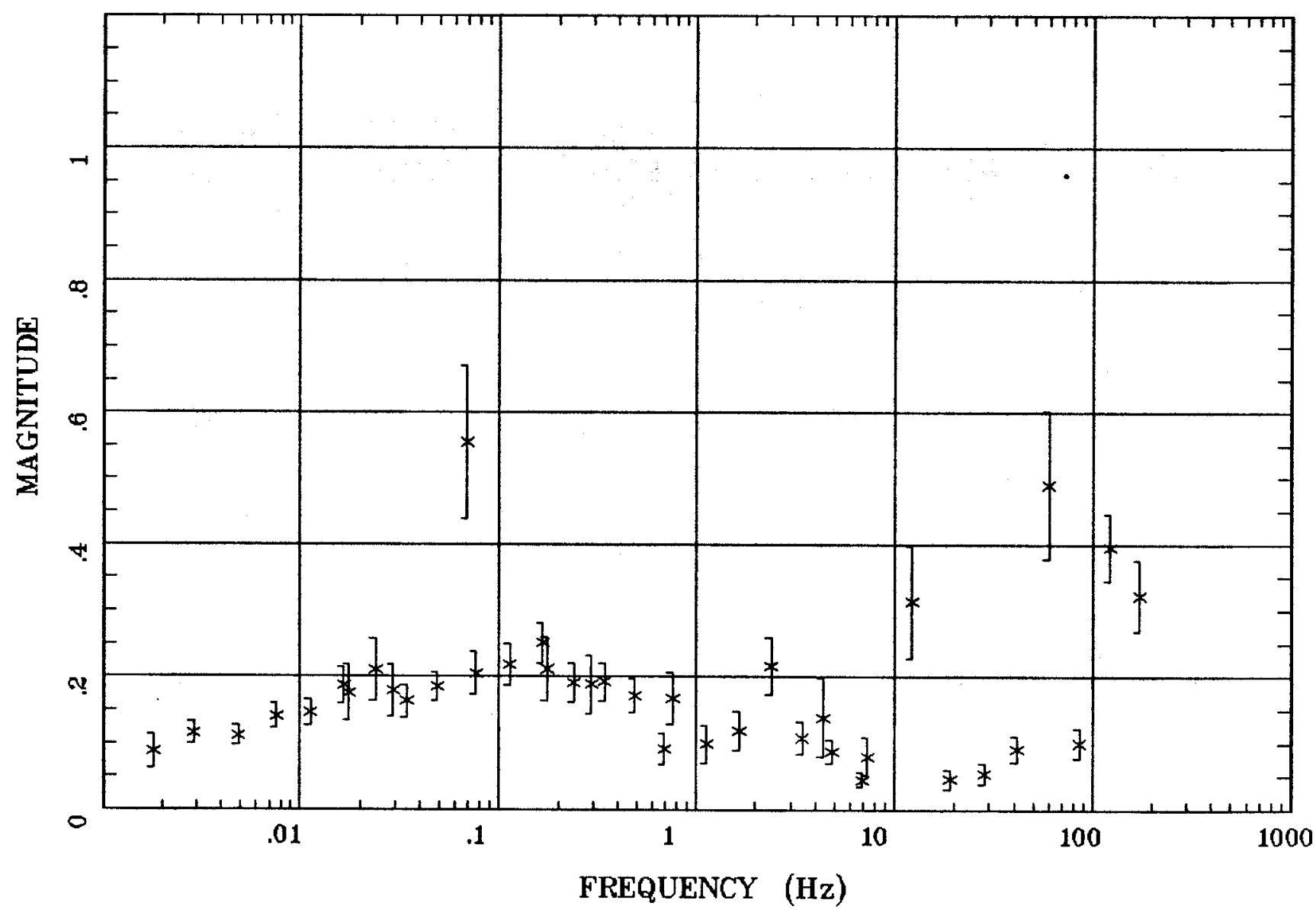

Client:

Remote: E local ref.

Acquired: $09: 2$ Jul 15, 1997 Survey Co:
Rotation:

Filename: nn23a.all

Channels: Ch1 Ch2 Ch3 Ch4 Ch5 Ch6 Ch7 Plotted: 08:36 Dec 08, 2000

$<$ EMI - ElectroMagnetic Instruments 


\section{TIPPER STRIKE}

Battle Mtn.

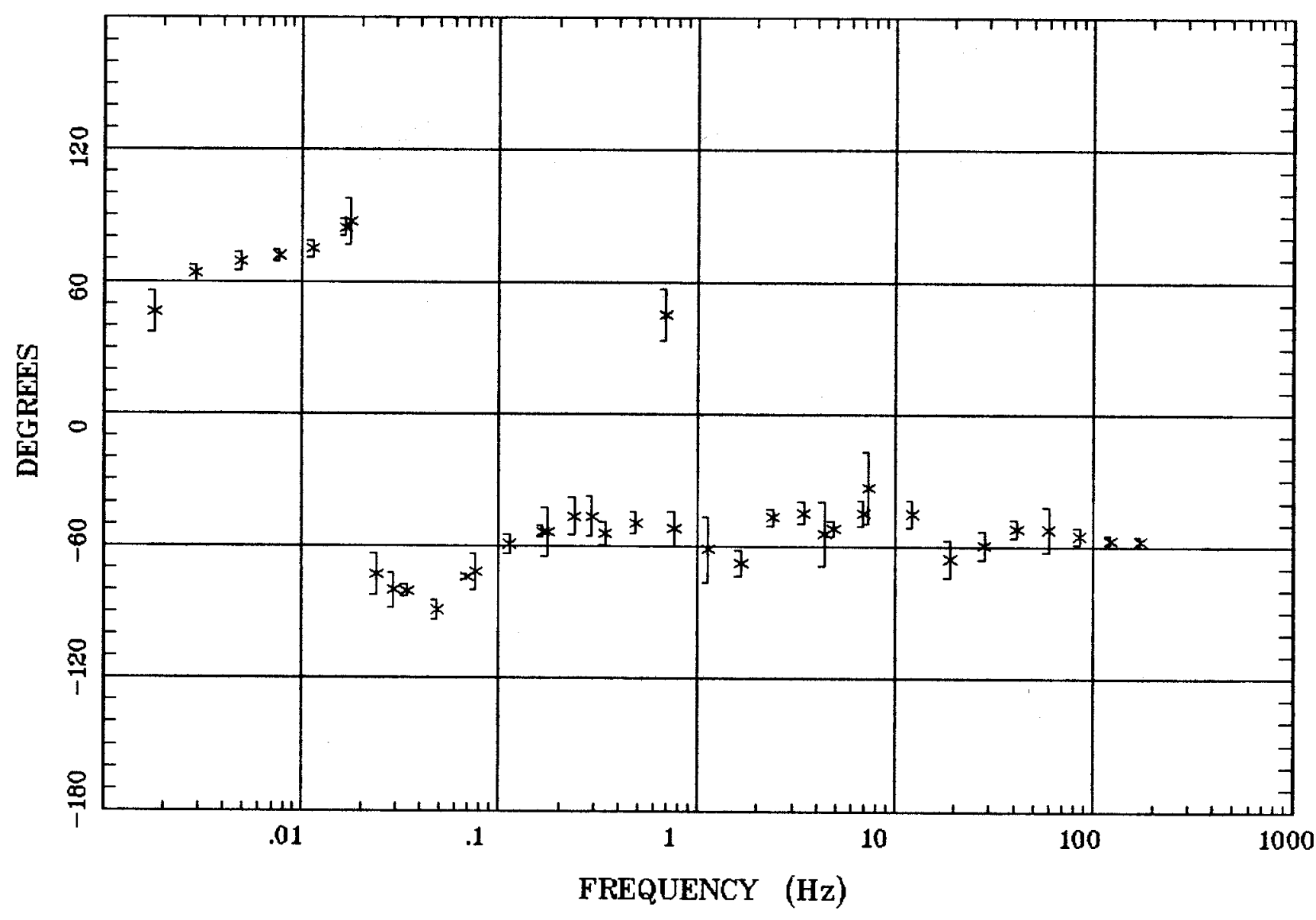

Client:

Remote: E local ref.

Acquired: 09:2 Jul 15, 1997 Survey Co:
Rotation:

Filename: nn23a.all

Channels: Ch1 Ch2 Ch3 Ch4 Ch5 Ch6 Ch7 Plotted: 08:36 Dec 08, 2000

$<$ EMI - ElectroMagnetic Instruments 


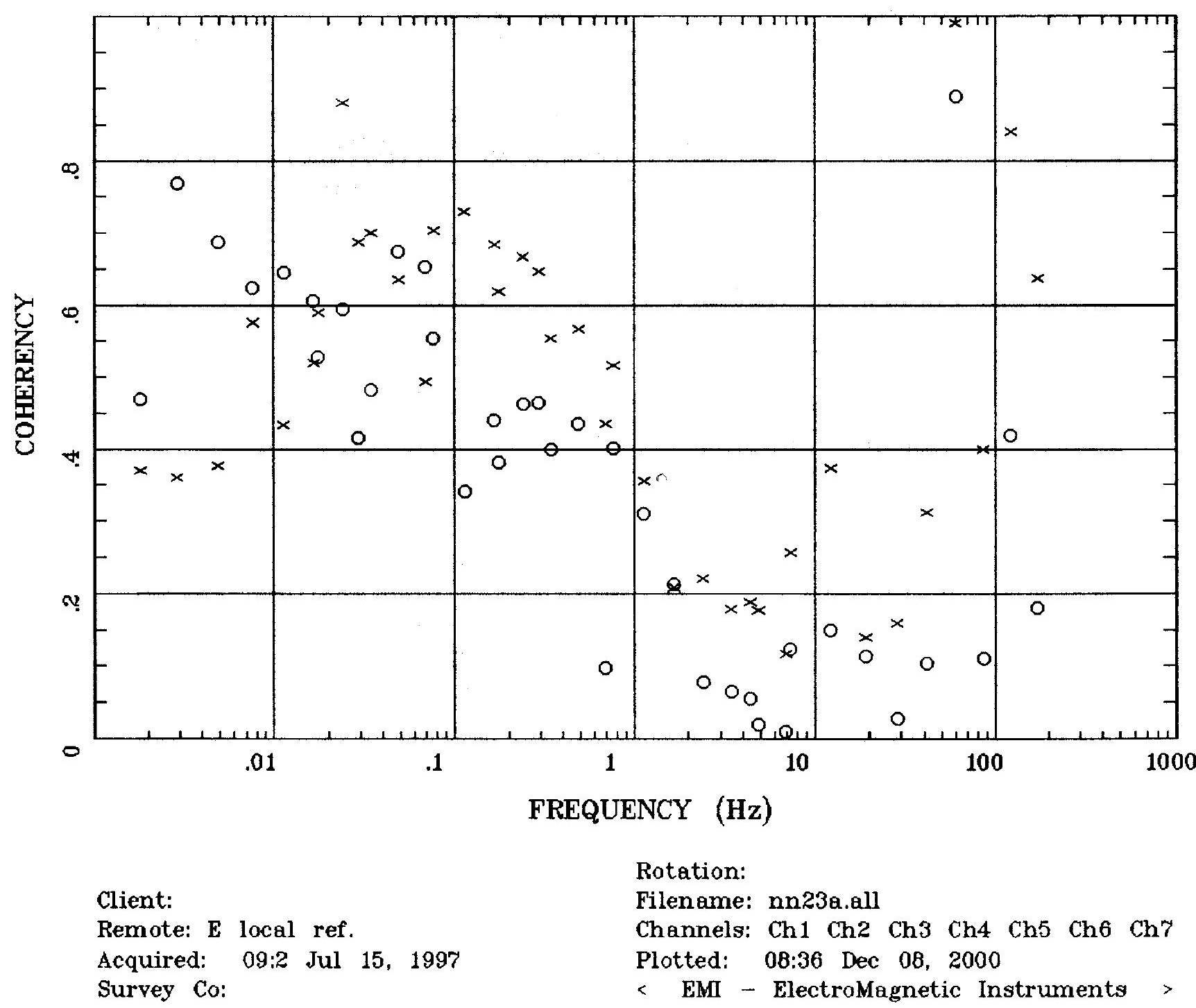


Station 21

APPARENT RESISTIVITY

...Battle Mtn...

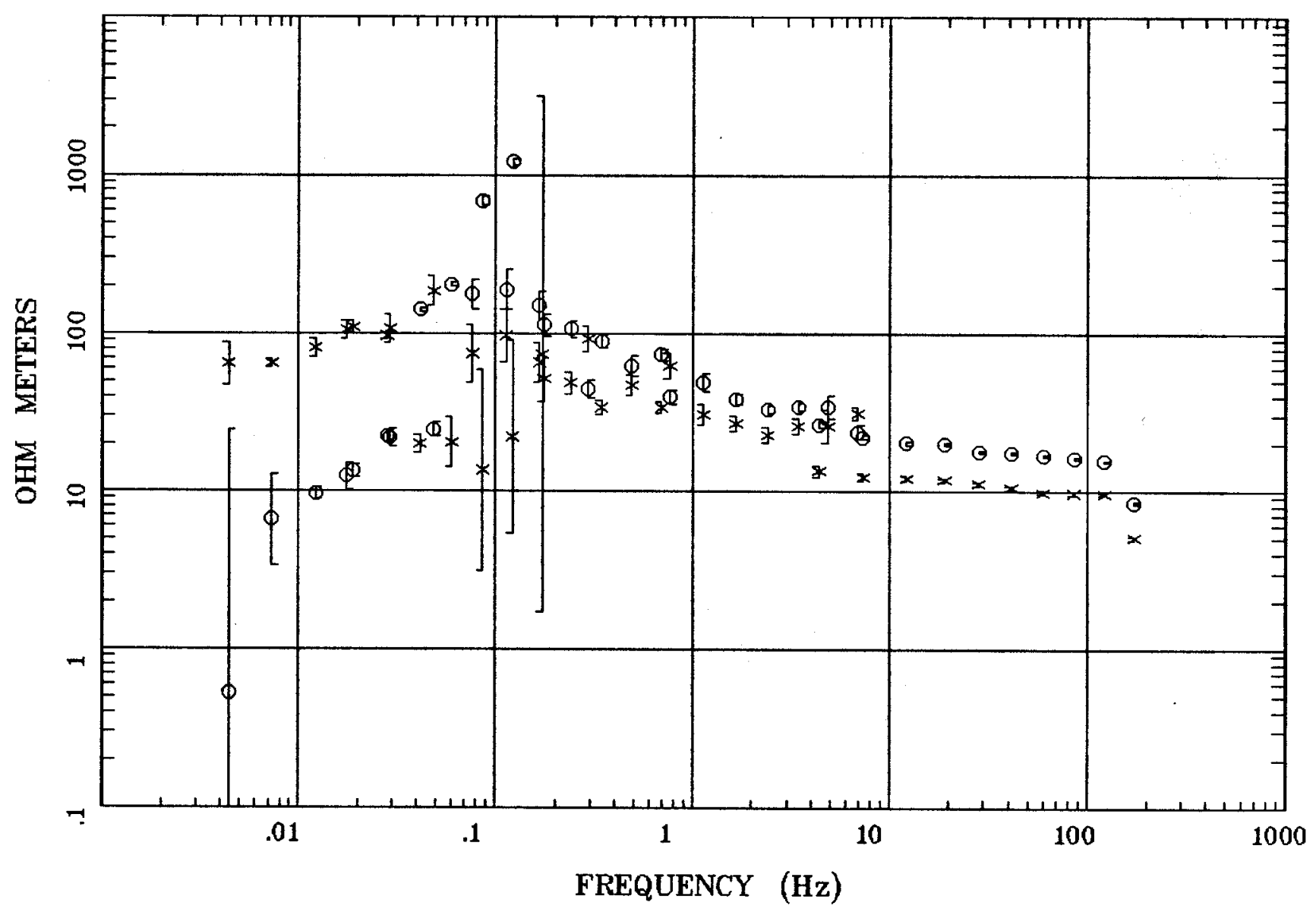

Client:

Remote: E local ref.

Acquired: 16:4 Jul 23, 1997

Survey Co:
Rotation:

Filename: nn21.all

Channels: Ch6 Ch7 Ch8 Ch9 Ch10Ch1 Ch2

Plotted: 08:33 Dec 08, 2000

$<$ EMI - ElectroMagnetic Instruments 
IMPEDANCE PHASE

...Battle Mtn...

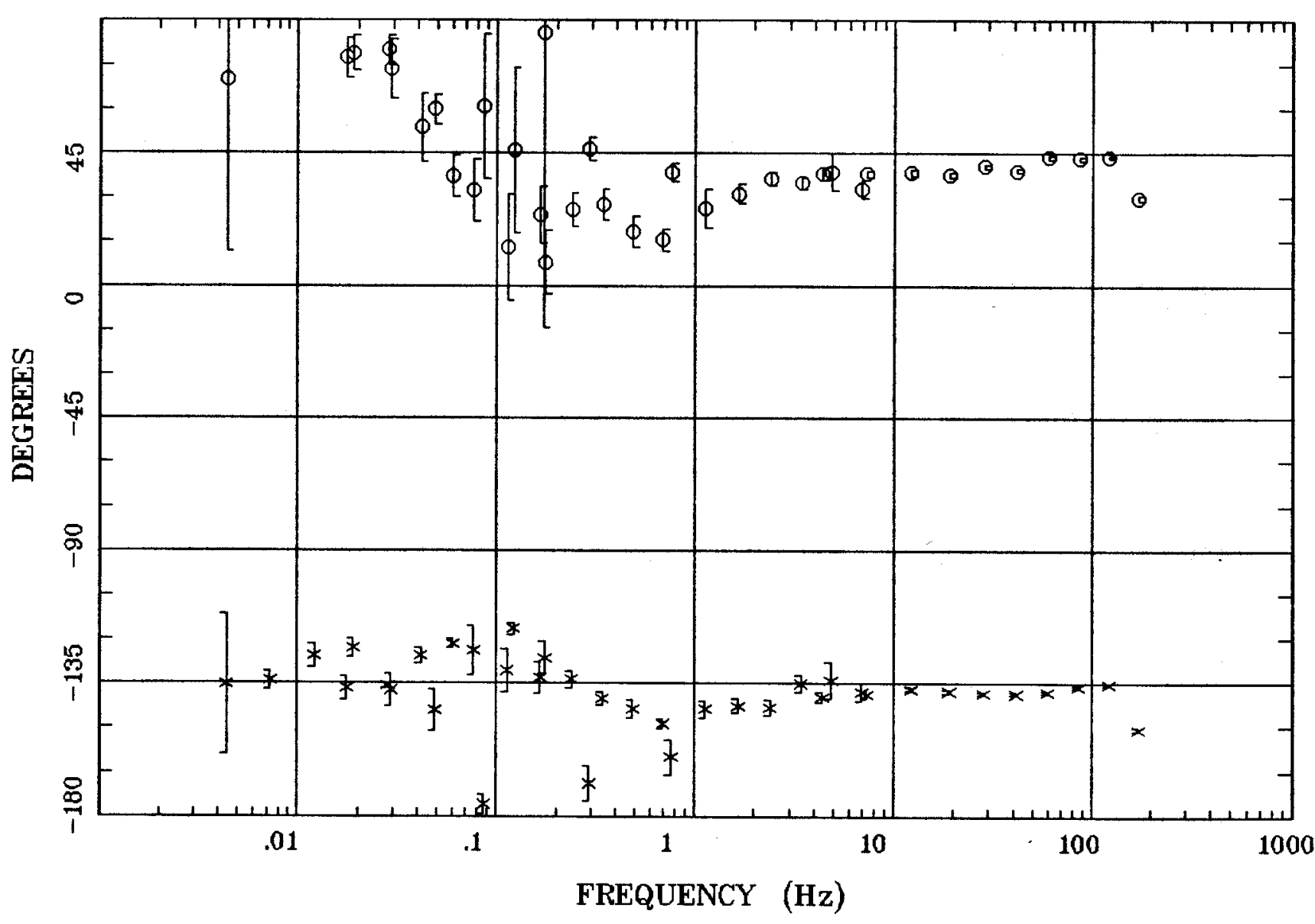

Client:

Remote: E local ref. Acquired: 16:4 Jul 23, 1997 Survey Co:
Rotation:

Filename: nnz1.all

Channels: Ch6 Ch7 Ch8 ch9 Ch10ch1 Ch2 Plotted: 08:33 Dec 08, 2000

$<$ EMI - ElectroMagnetic Instruments > 
Station 21

ROTATION ANGLE

...Battle Mtn...

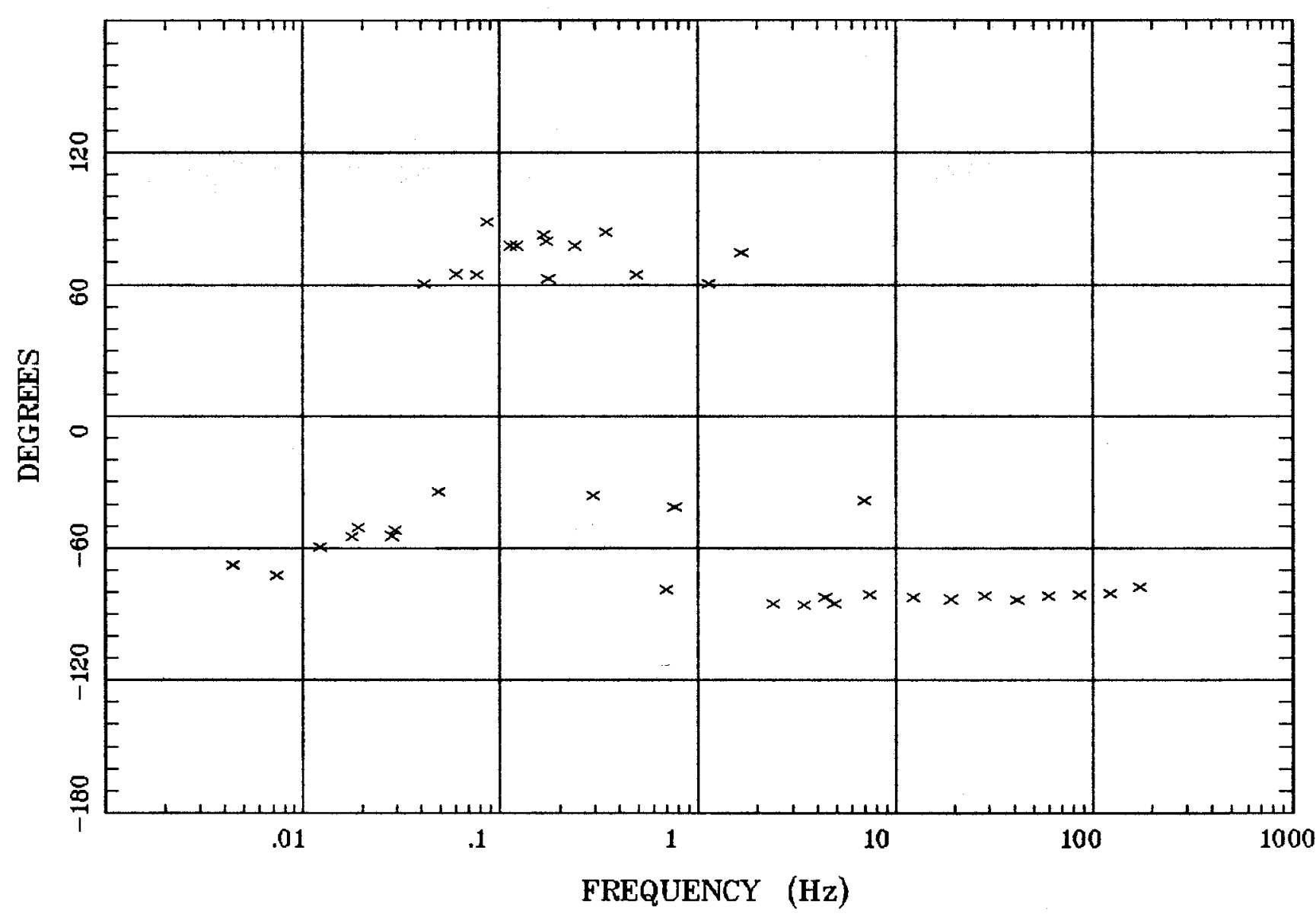

Client:

Remote: E local ref.

Acquired: 16:4 Jul 23, 1997 Survey Co:

Rotation:

Filename: nn21.all

Channels: Ch6 Ch7 Ch8 Ch9 Ch10Ch1 Ch2

Plotted: 08:33 Dec 08, 2000

$<$ EMI - ElectroMagnetic Instruments 


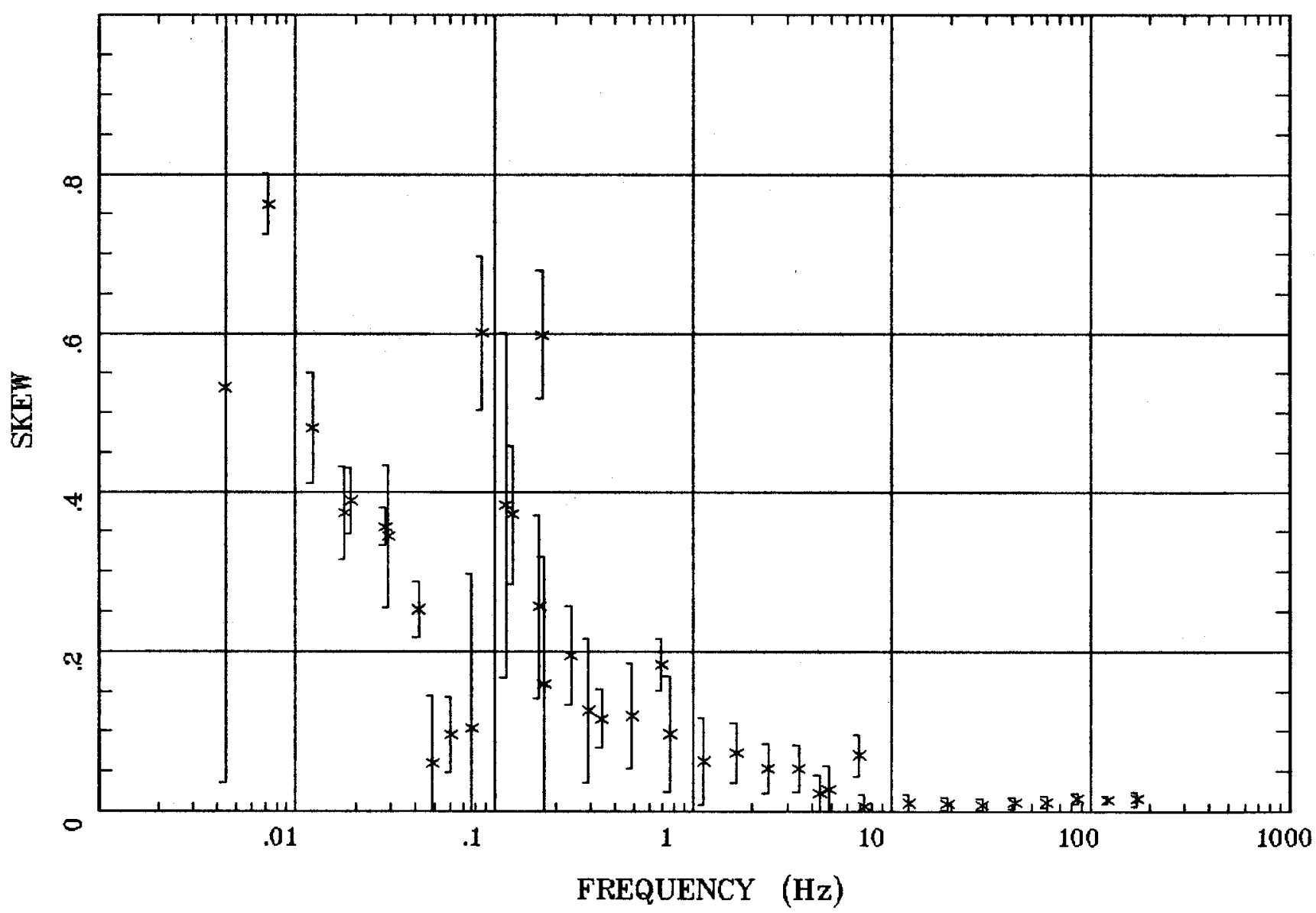

Client:

Remote: E local ref.

Acquired: 16:4 Jul 23, 1997

Survey Co:

\section{Rotation:}

Filename: nn21.all

Channels: Ch6 Ch7 Ch8 Ch9 Ch10Ch1 Ch2

Plotted: 08:33 Dec 08, 2000

< EMI - ElectroMagnetic Instruments 


\section{E MULT Coh.}

...Battle Mtn...

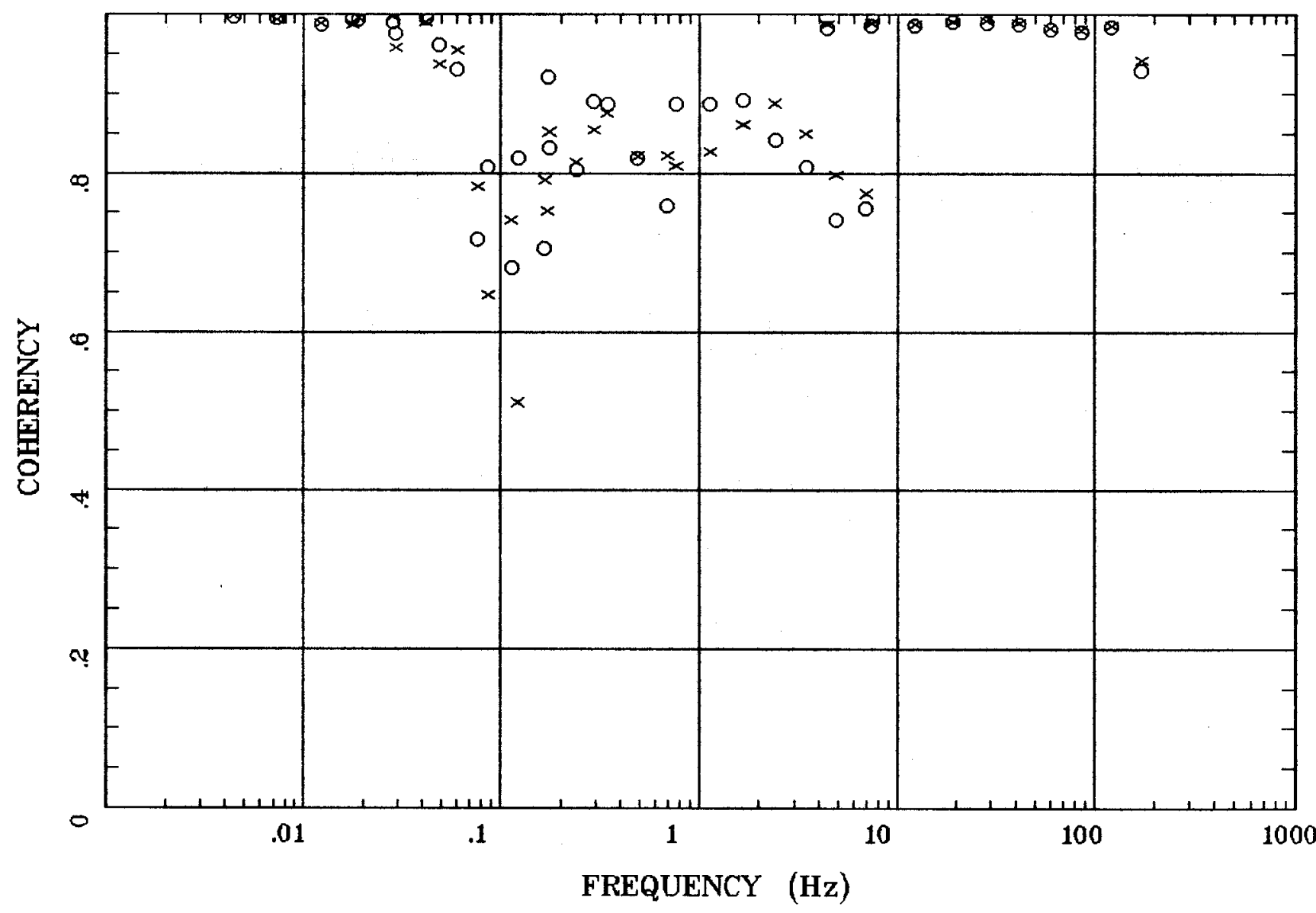

Client:

Remote: E local ref.

Acquired: 16:4 Jul 23, 1997 Survey Co:
Rotation:

Filename: nn21.all

Channels: Ch6 Ch7 Ch8 Ch9 Ch10ch1 Ch2 Plotted: 08:33 Dec 08, 2000

< EMI - ElectroMagnetic Instruments 


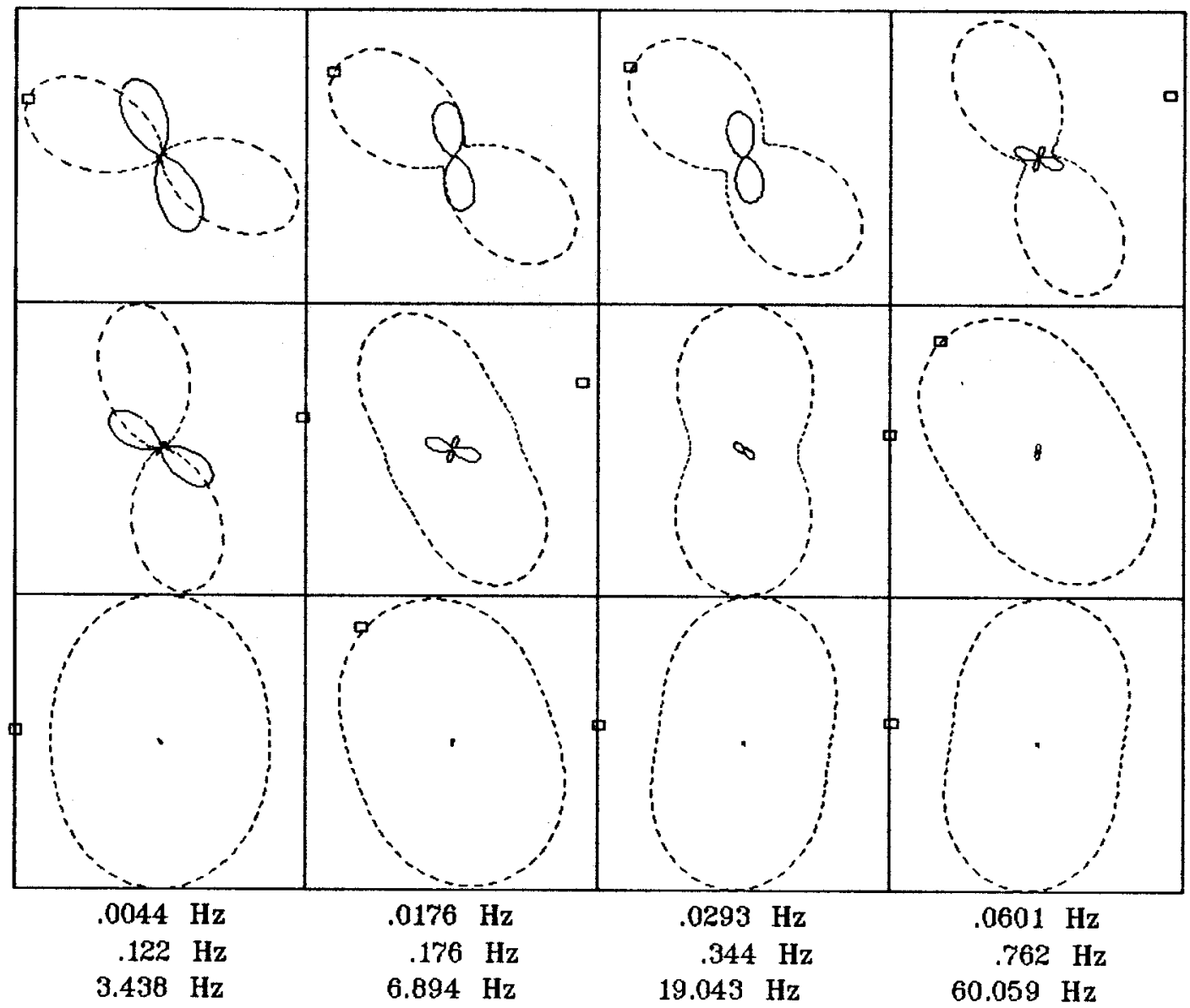

Client:

Remote: E local ref.

Acquired: 16:4 Jul 23, 1997

Survey Co:
Rotation:

Filename: nn21.all

Channels: Ch6 Ch7 Ch8 Ch9 Ch10Ch1 Ch2 Plotted: 08:33 Dec 08, 2000

< EMI - ElectroMagnetic Instruments > 


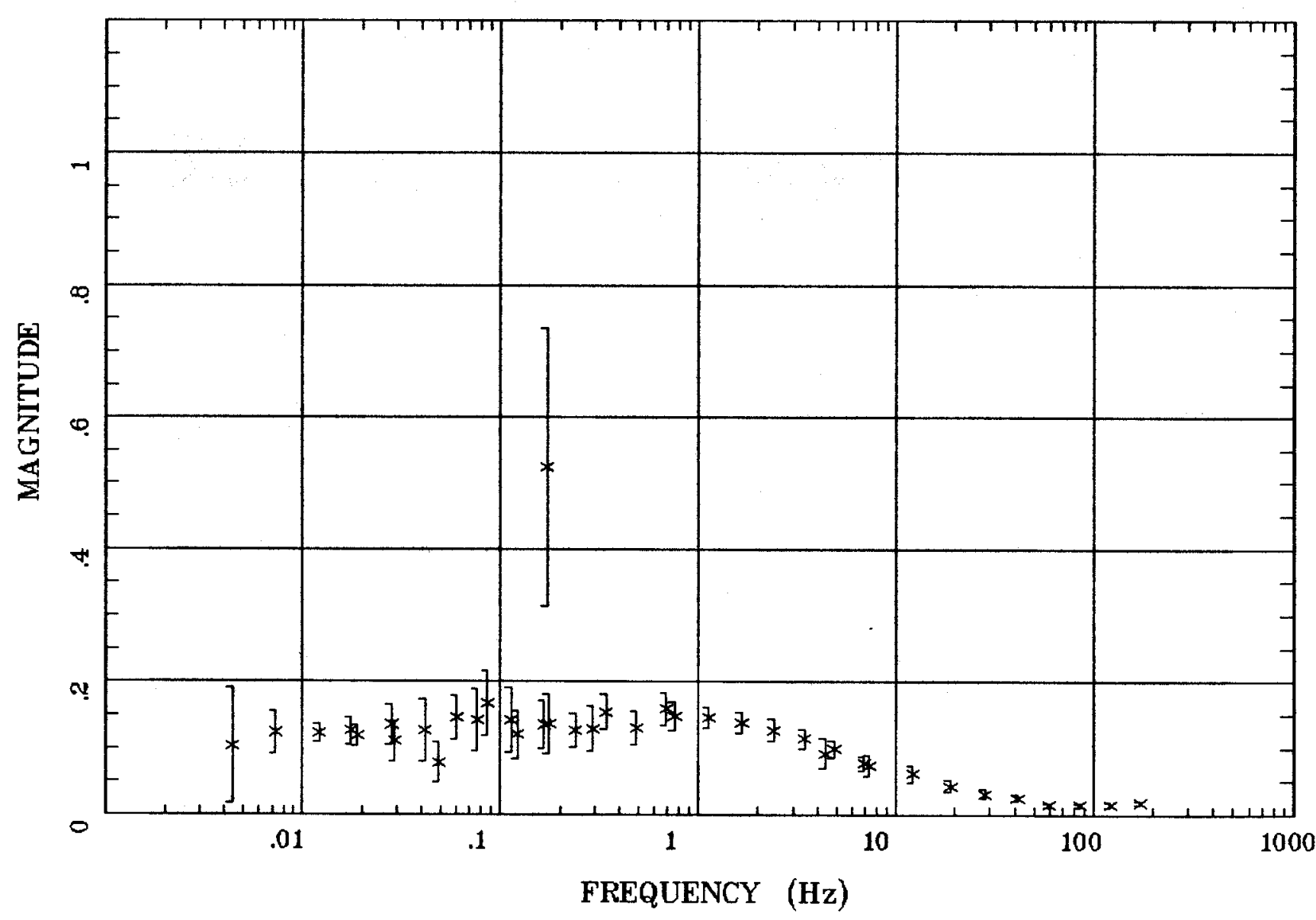

Client:

Remote: E local ref.

Acquired: $16: 4$ Jul 23, 1997 Survey Co:
Rotation:

Filename: nn21.all

Channels: Ch6 Ch' Ch8 Chg Ch10Ch1 Ch2 Plotted: 08:33 Dec 08, 2000

< EMI - ElectroMagnetic Instruments 


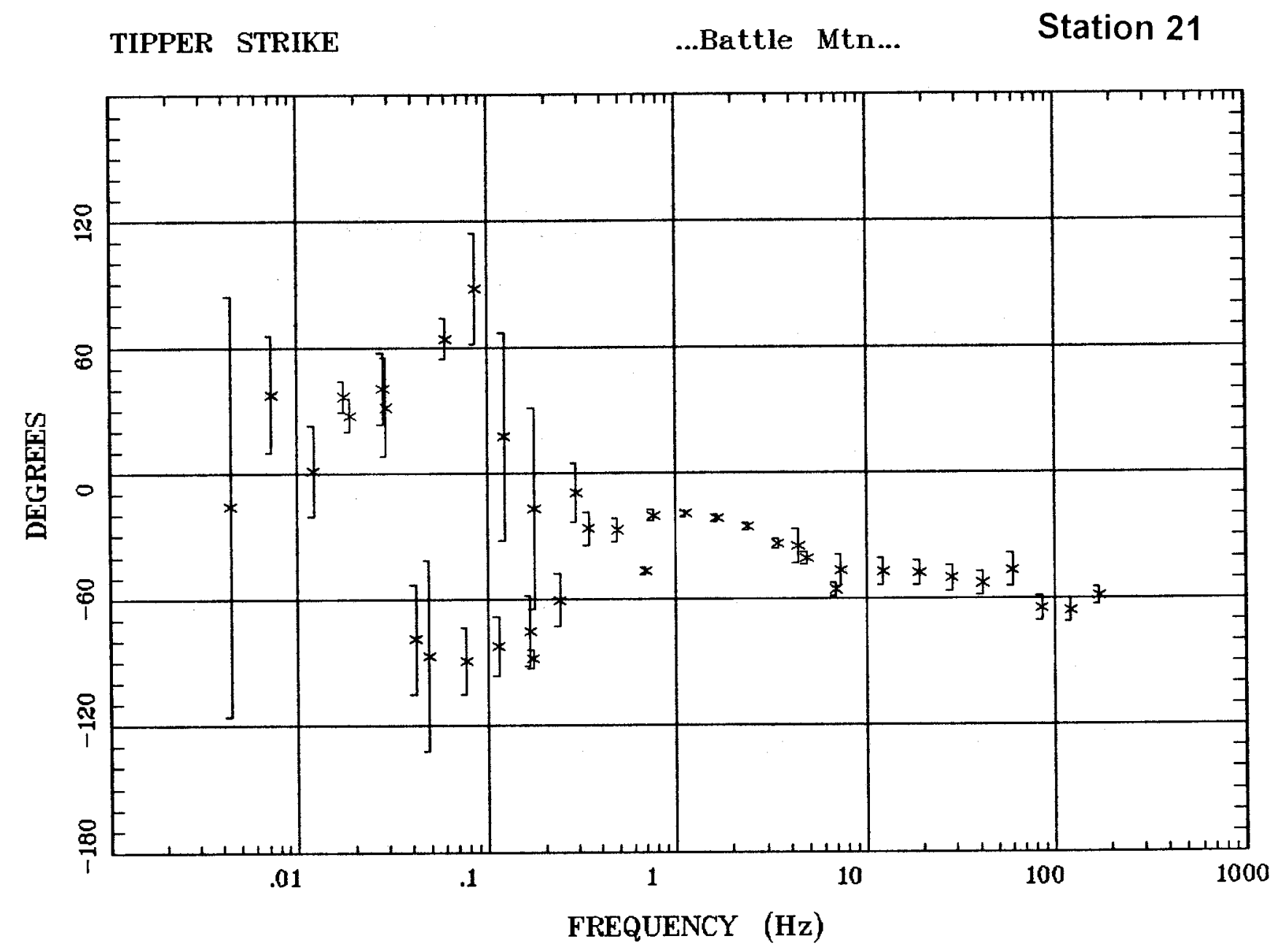

Client:

Remote: E local ref.

Acquired: 16:4 Jul 23, 1997 Survey Co:

Rotation:

Filename: nn21.all

Channels: Ch6 Ch7 Ch8 Ch9 Ch10Ch1 Ch2

Plotted: 08:33 Dec 08, 2000

< EMI - ElectroMagnetic Instruments > 


\section{HzHx.x Coh HzHy.o}

Station 21

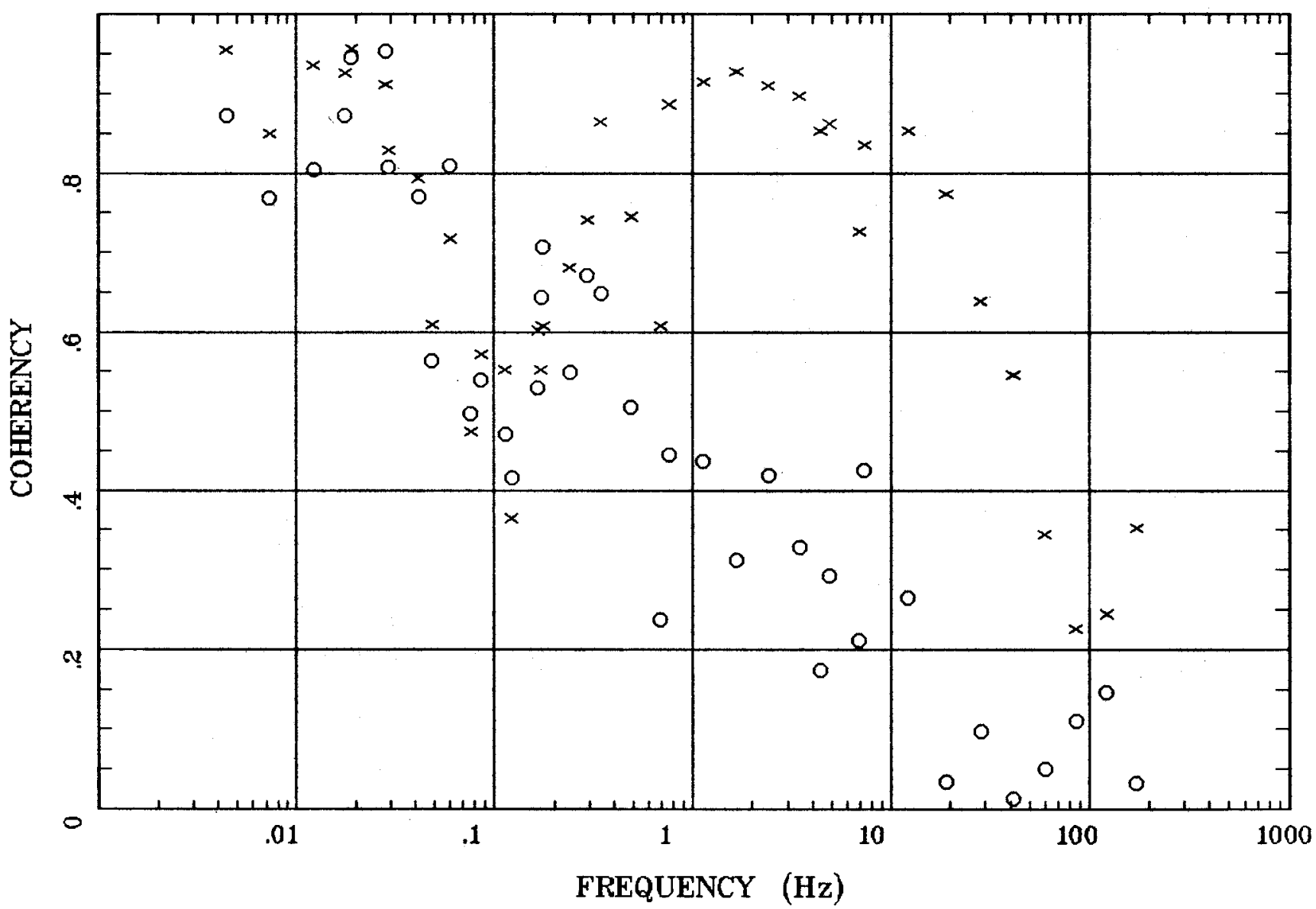

Client:

Remote: E local ref.

Acquired: 16:4 Jul 23, 1997

Filename: nn21.all

Survey Co:

Channels: Ch6 Ch7 Ch8 Ch9 Ch10Ch1 Ch2

Plotted: 08:34 Dec 08, 2000

< EMI - ElectroMagnetic Instruments > 


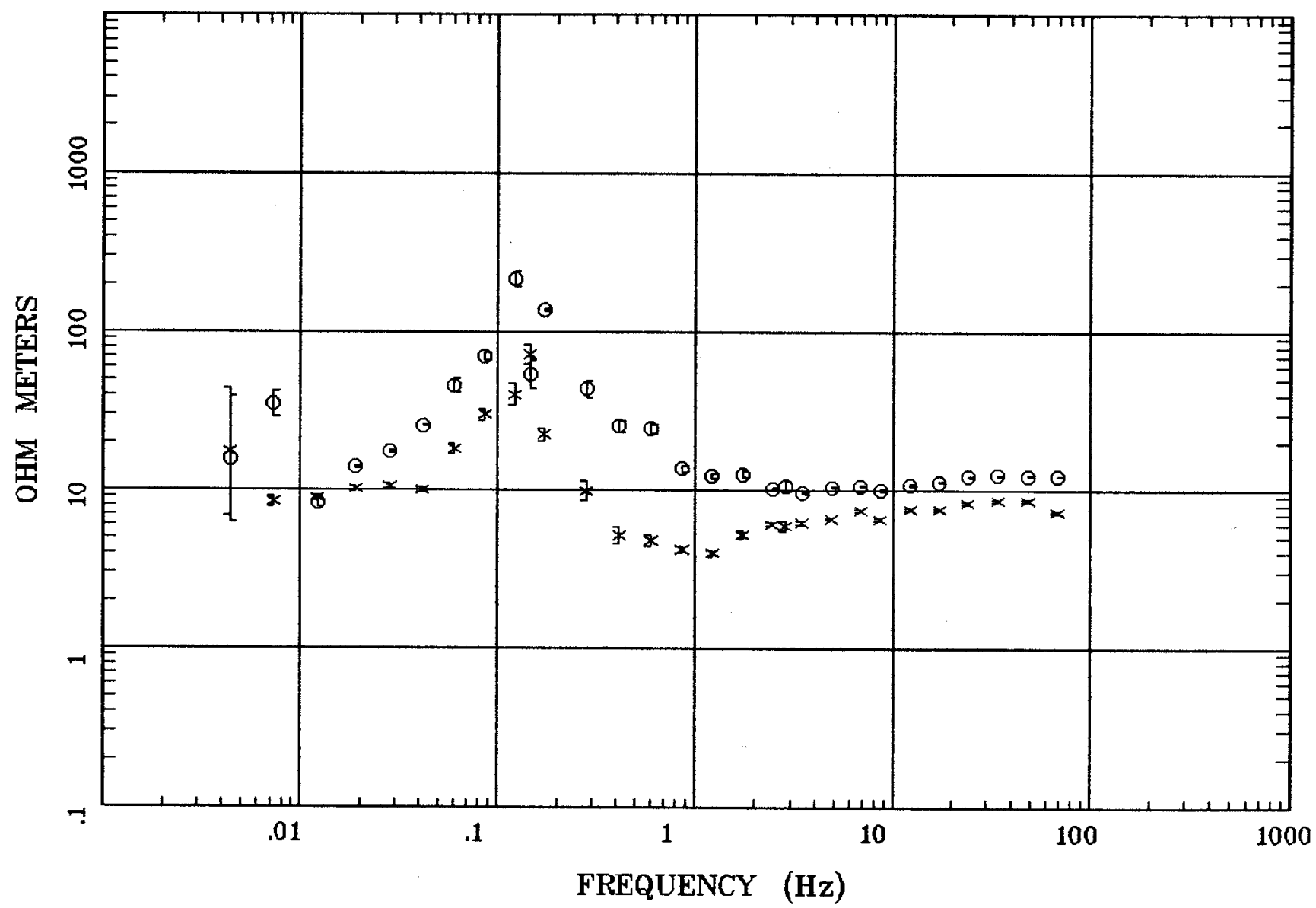

Client:

Remote: e-field local ref Acquired: 10:2 Jul 12, 1997 Survey Co:
Rotation:

Filename: nn24c.all

Channels: Ch1 Ch2 Ch3 Ch4 Ch5 Ch6 Ch7 Platted: 08:37 Dec 08, 2000

< EMI - ElectroMagnetic Instruments > 
Station 24A

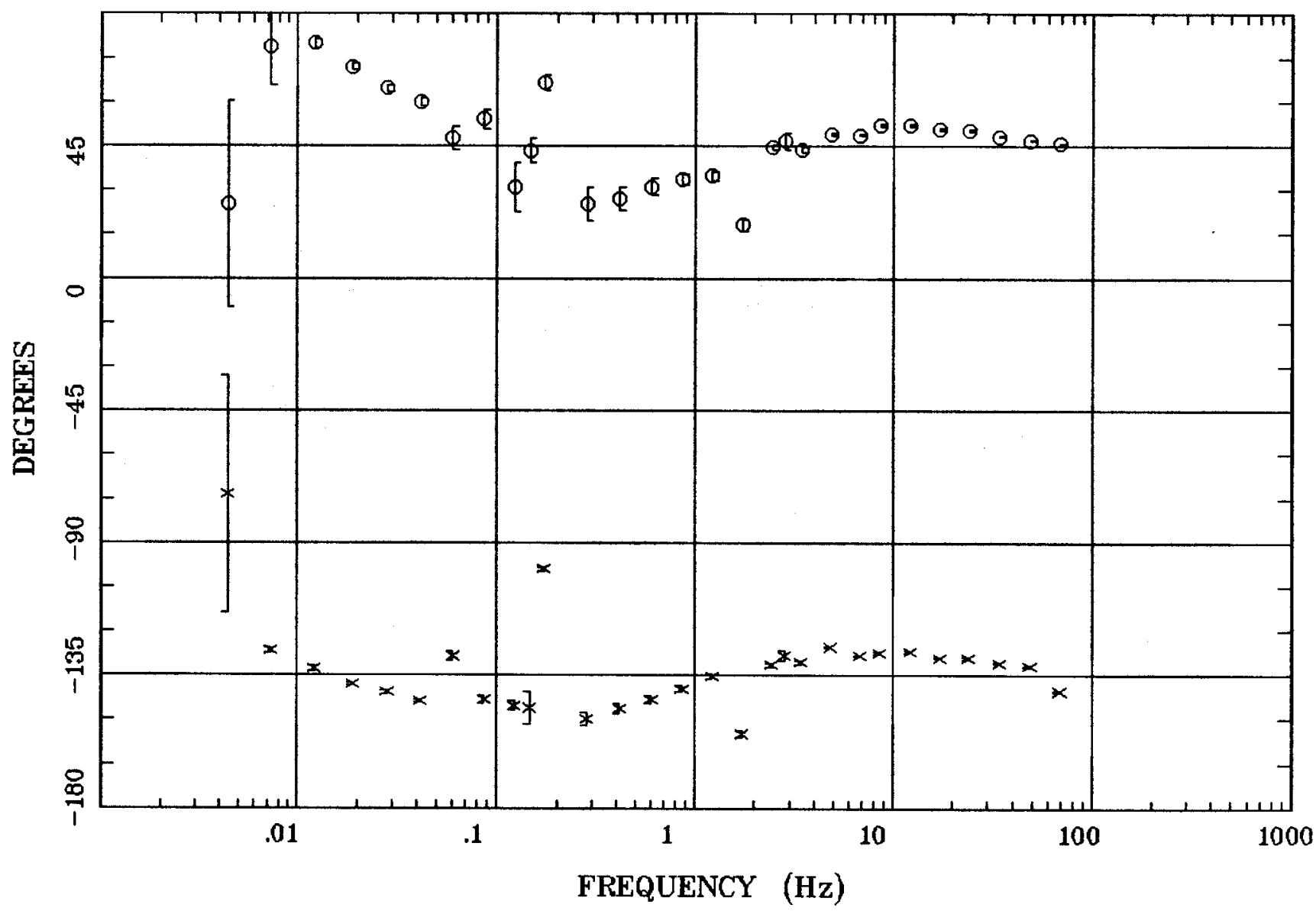

Client:

Remote: e-field local ref Acquired: 10:2 Jul 12, 1997 Survey Co:

\section{Rotation:}

Filename: nn24c.all

Channels: Ch1 Ch2 Ch3 Ch4 Ch5 Ch6 Ch7 Plotted: 08:37 Dec 08, 2000

$<$ EMI - ElectroMagnetic Instruments 


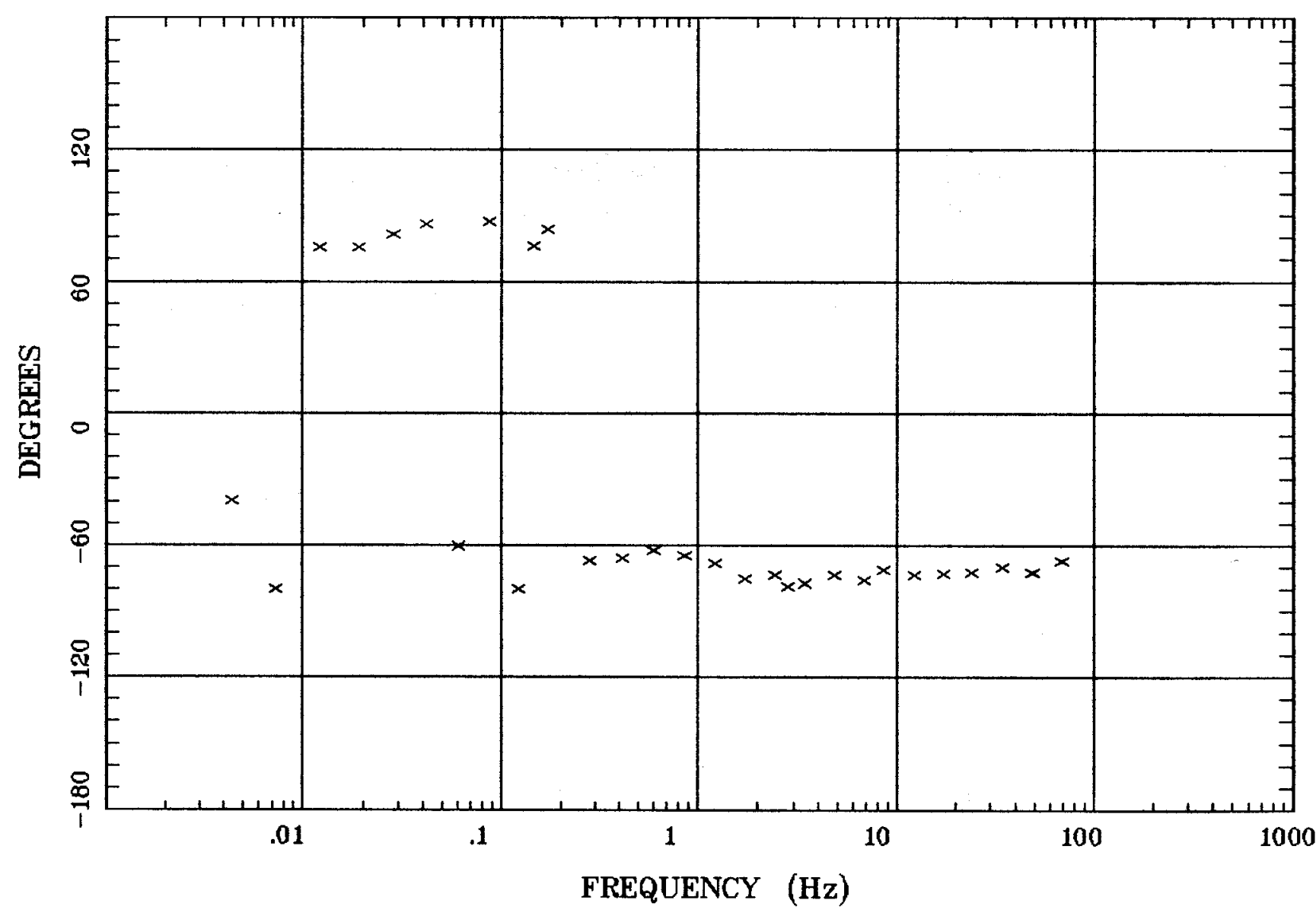

Client:

Remote: e-field local ref Acquired: 10:2 Jul 12, 1997 Survey Co:
Rotation:

Filename: nn24c.all

Channels: Ch1 Ch2 Ch3 Ch4 Ch5 Ch6 Ch7 Plotted: 08:37 Dec 08, 2000

< EMI - ElectroMagnetic Instruments 


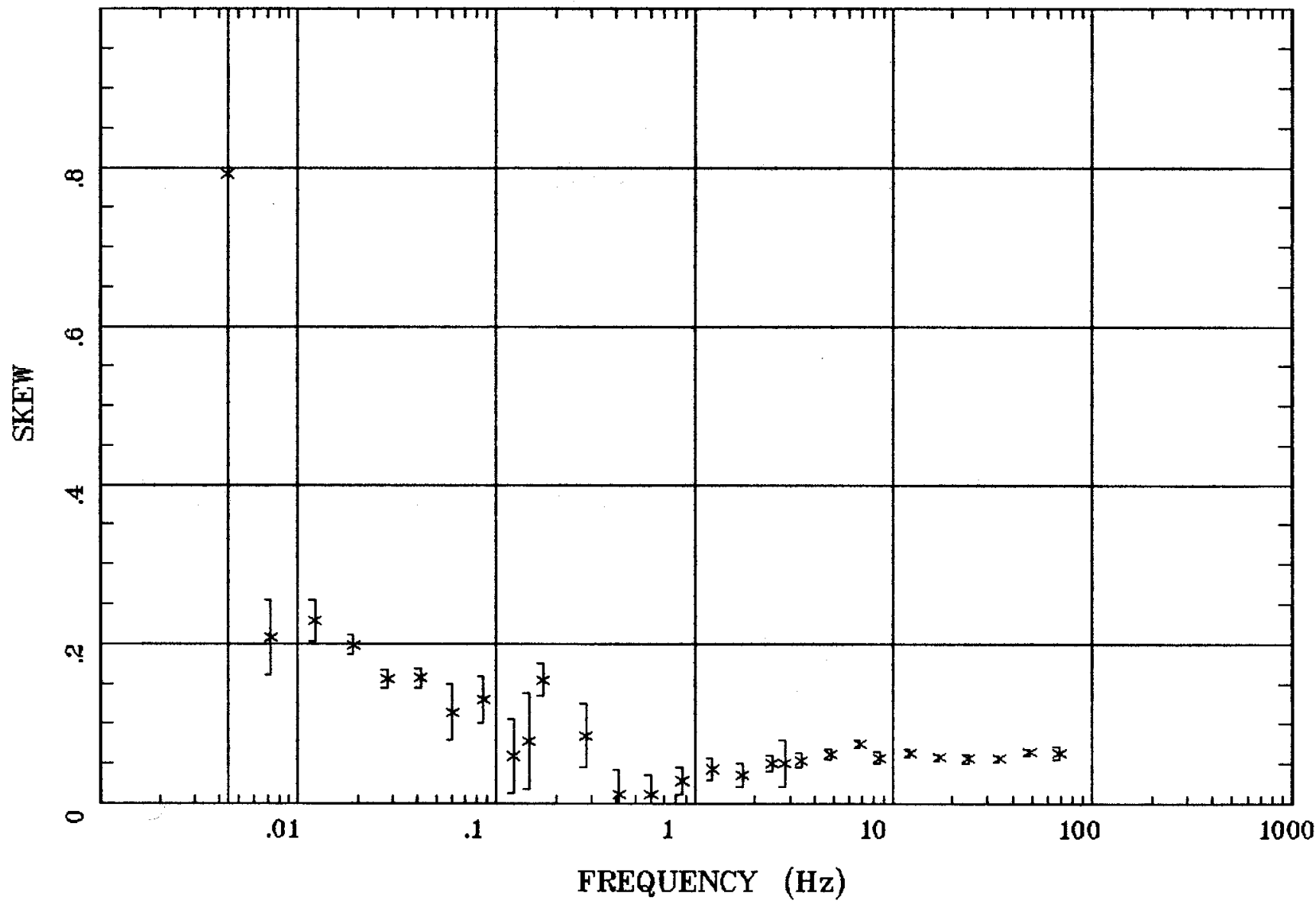

Client:

Remote: e-field local ref

Acquired: 10:2 Jul 12, 1997 Survey Co:
Rotation:

Filename: nn24c.all

Channels: Ch1 Ch2 Ch3 Ch4 Ch5 Ch6 Ch7

Plotted: 08:37 Dec 08, 2000

< EMI - ElectroMagnetic Instruments 


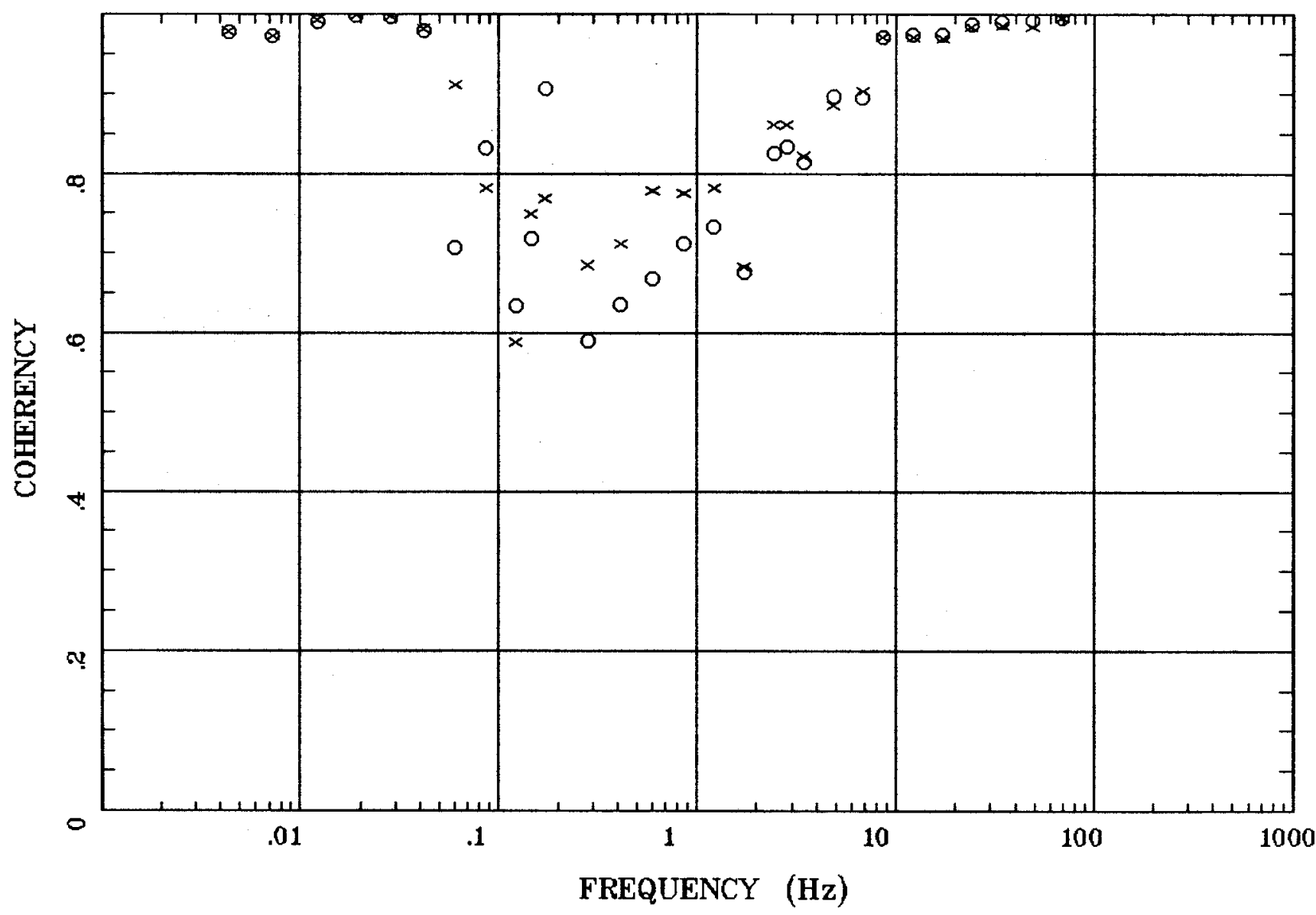

Client:

Remote: e-field local ref Acquired: 10:2 Jul 12, 1997 Survey Co:
Rotation:

Filename: nn24c.all

Channels: Ch1 Ch2 Ch3 Ch4 Ch5 Ch6 Ch7 Plotted: 08:37 Dec 08, 2000

$<$ EMI - ElectroMagnetic Instruments 


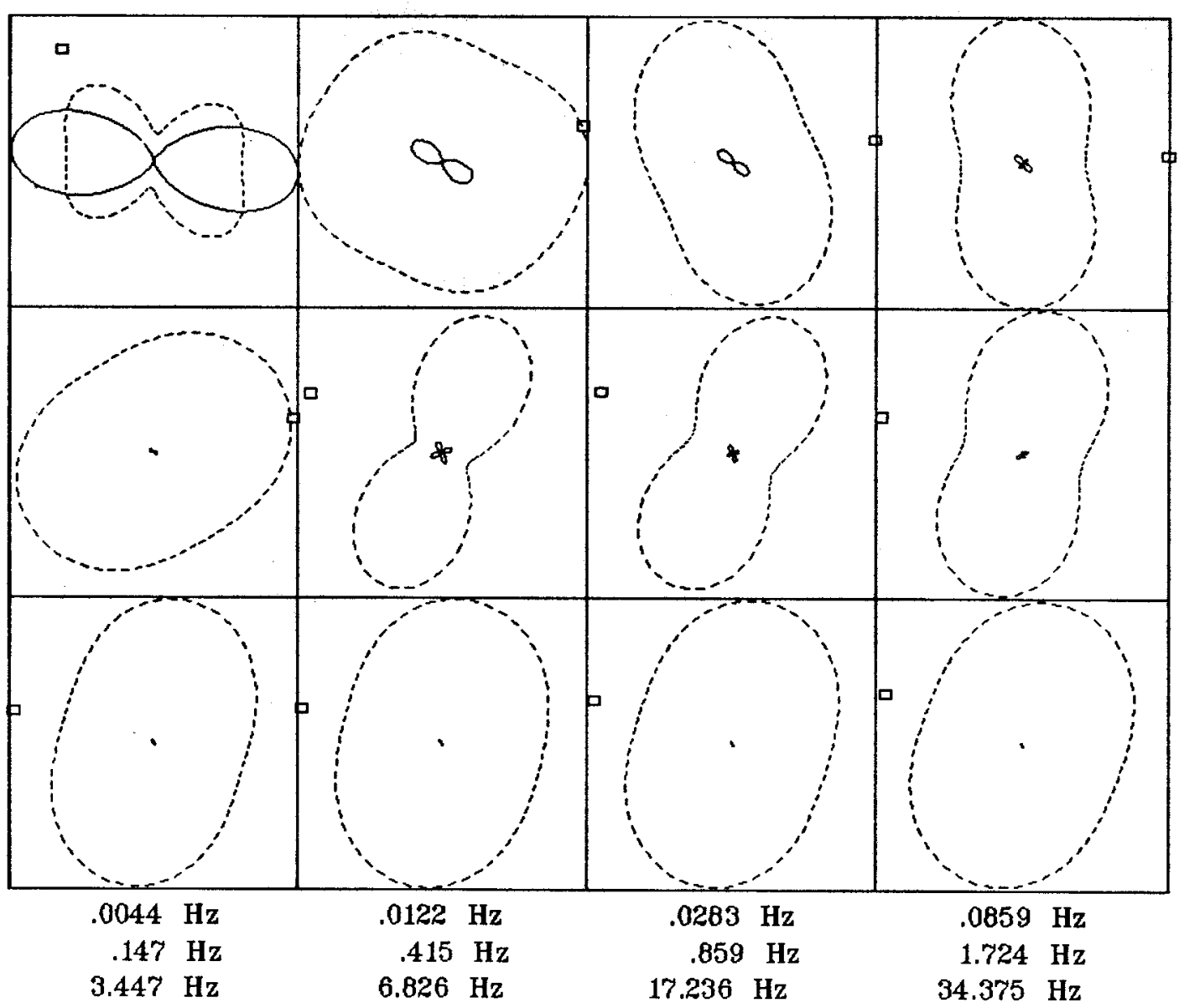

Client:

Remote: e-field local ref Acquired: 10:2 Jul 12, 1997 Survey Co:
Battle Mtn.

Rotation:

Filename: nn24c.all

Channels: Ch1 Ch2 Ch3 Ch4 Ch5 Ch6 Ch7

Plotted: 08:37 Dec 08, 2000

< EMI - ElectroMagnetic Instruments > 


\section{TIPPER MAGNITUDE}

Battle Mtn.

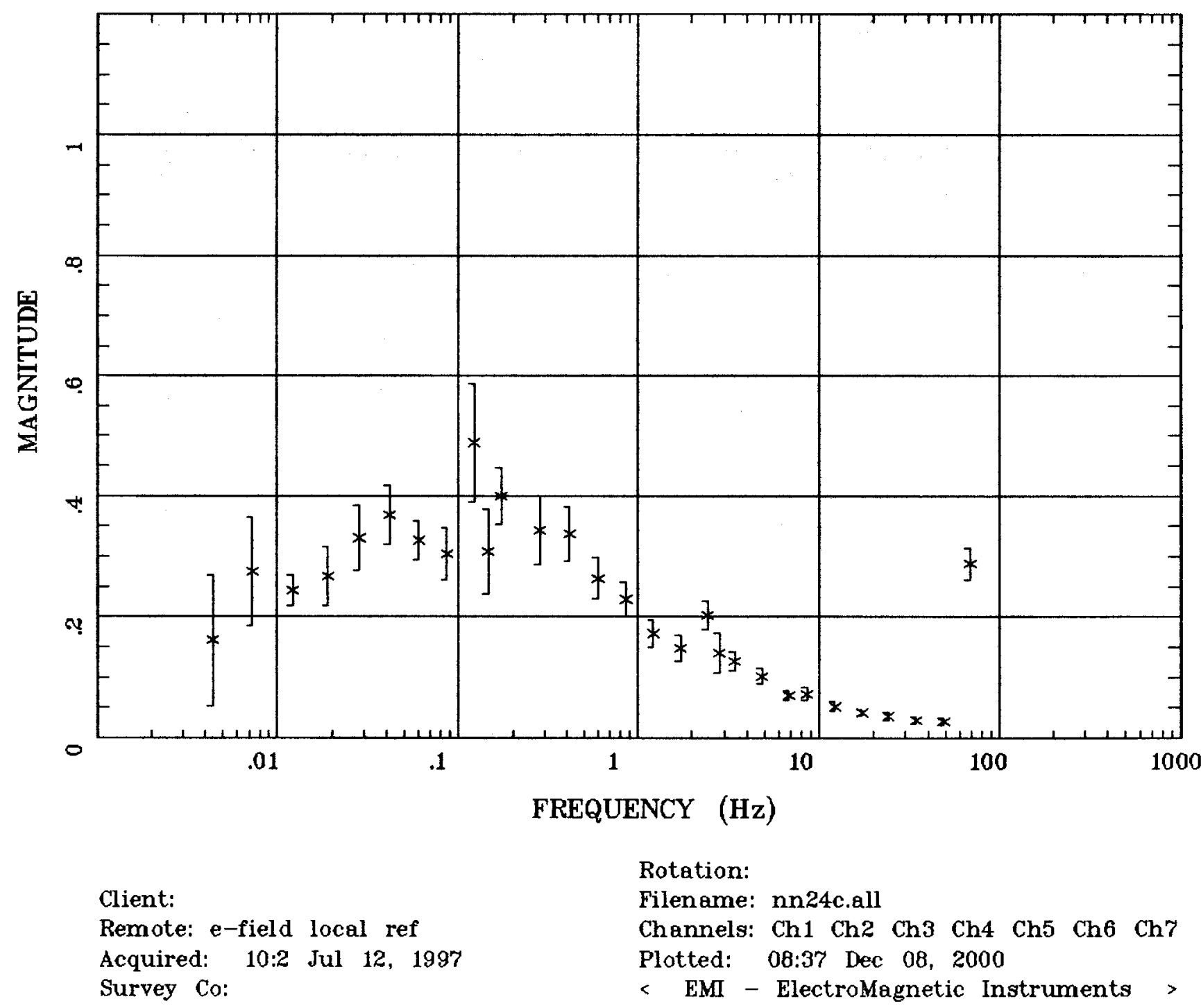




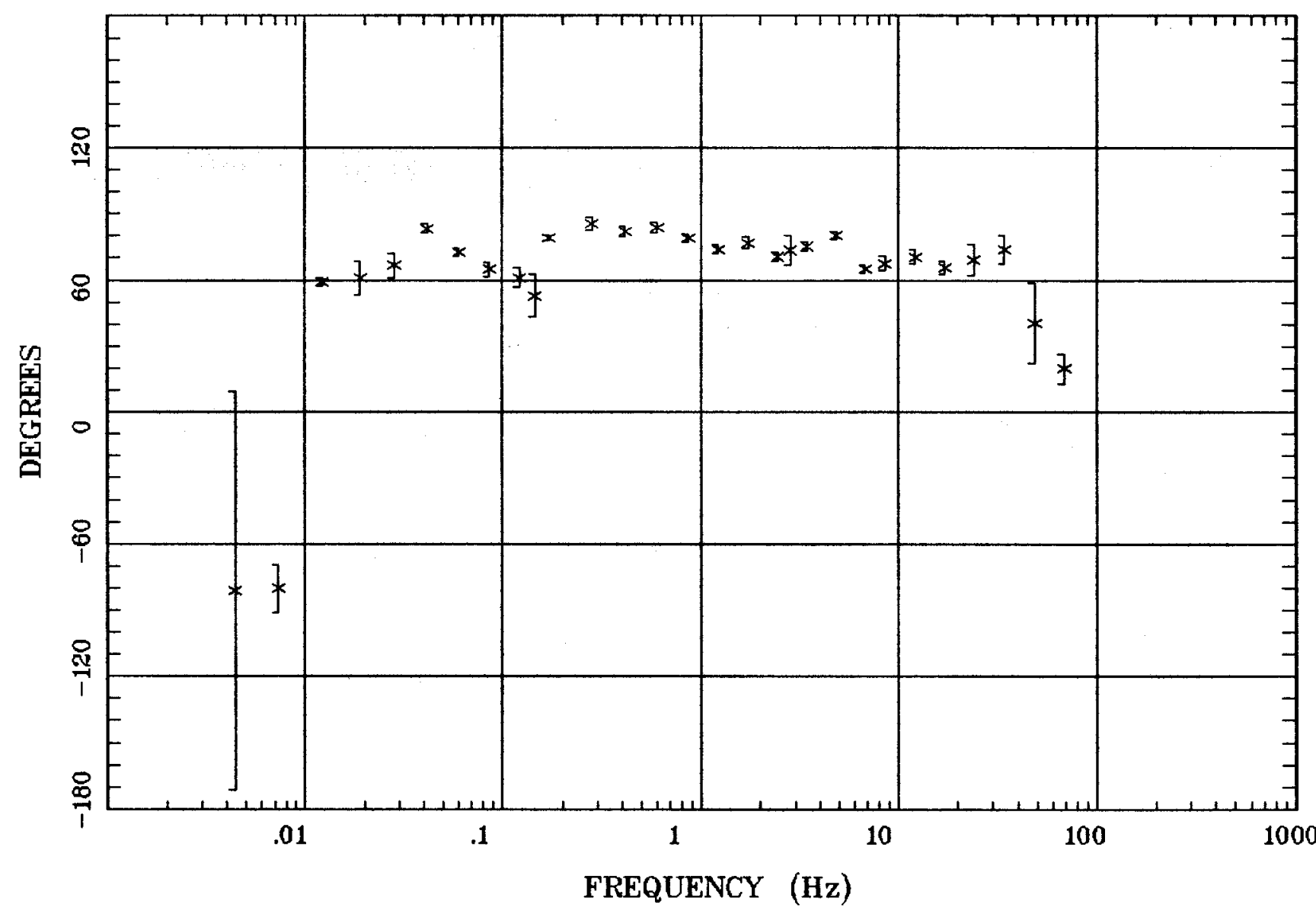

Client:

Remote: e-field local ref Acquired: 10:2 Jul 12, 1997 Survey Co:
Rotation:

Filename: nn24c.all

Channels: Ch1 Ch2 Ch3 Ch4 Ch5 Ch6 Ch7 Plotted: 08:37 Dec 08, 2000

\& EMI - ElectroMagnetic Instruments 

HzHx.x Coh HzHy.o
Battle Mtn.

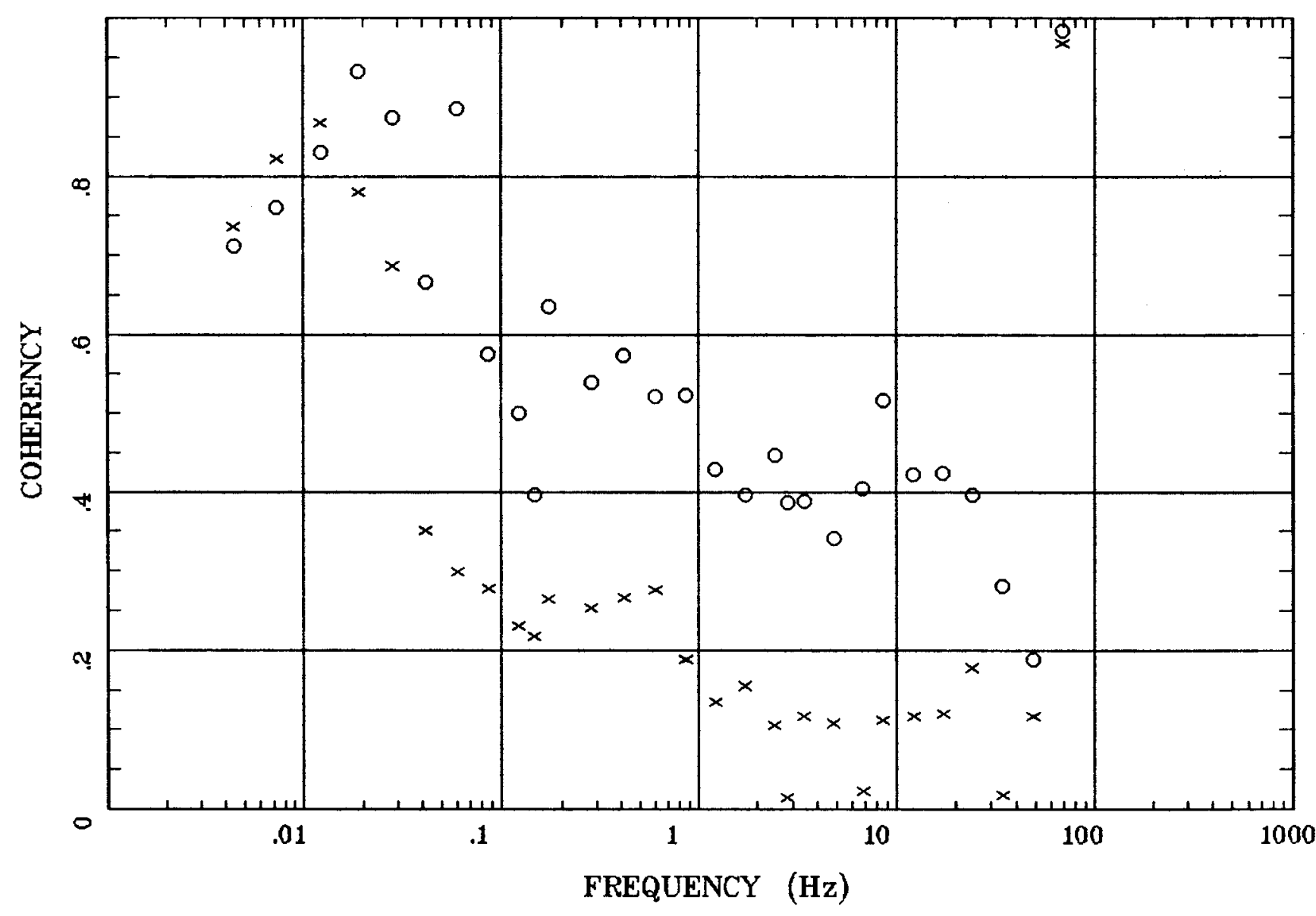

Client:

Remote: e-field local ref Acquired: 10:2 Jul 12, 1997 Survey Co:
Rotation:

Filename: nnz4c.all

Channels: Ch1 Ch2 Ch3 Ch4 Ch5 Ch6 Ch7

Plotted: 08:37 Dec 08, 2000

$<$ EMI - ElectroMagnetic Instruments 

APPARENT RESISTIVITY
Battle Mtn.

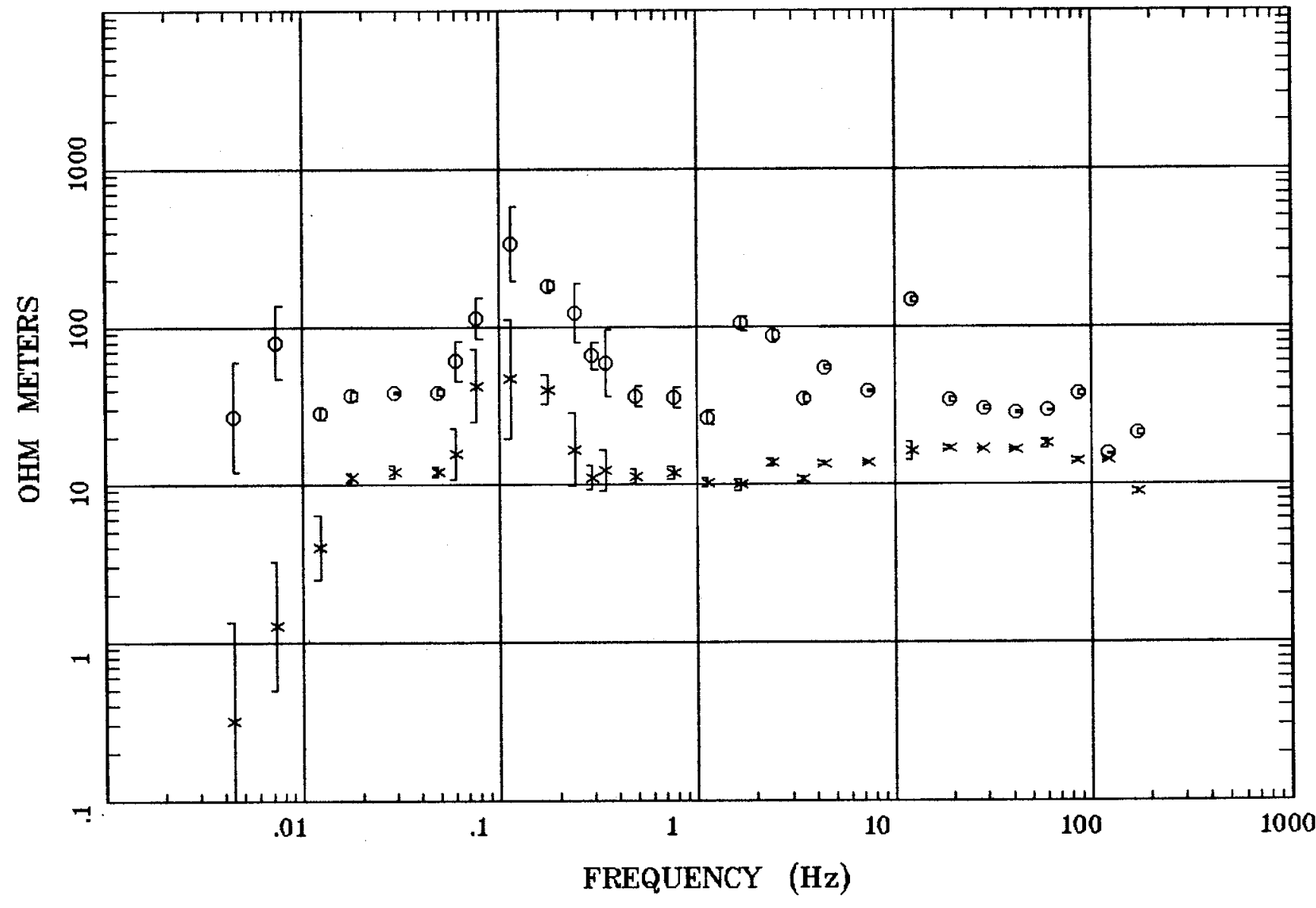

Client:

Remote: E local ref.

Acquired: 11:3 Jul 14, 1997

Survey Co:
Rotation:

Filename: nn25a.all

Channels: Ch1 Ch2 Ch3 Ch4 Ch5 Ch6 Ch7 Platted: 08:39 Dec 08, 2000

$<$ EMI - ElectroMagnetic Instruments 
IMPEDANCE PHASE

Battle Mtn.

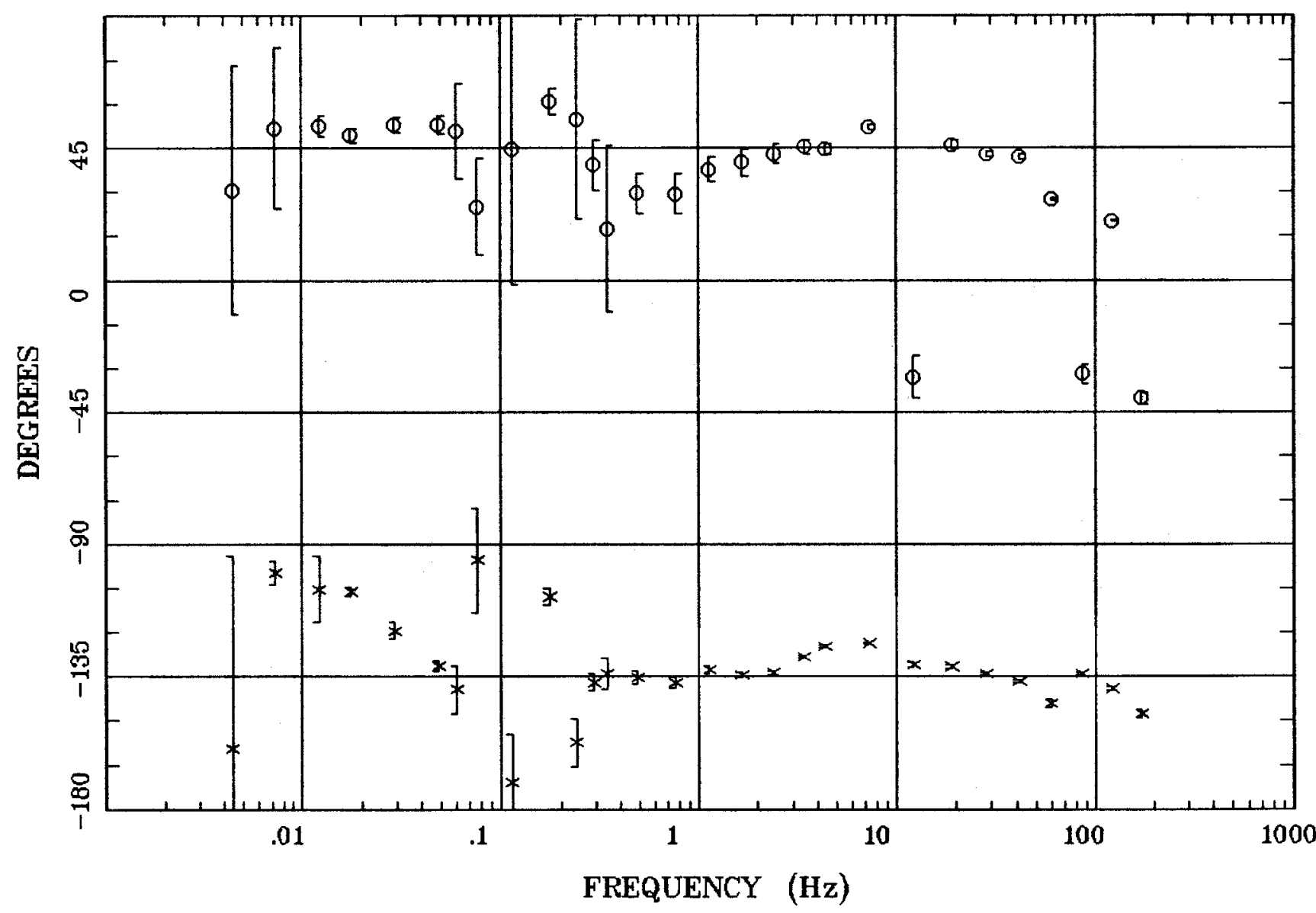

Client:

Remote: $\mathrm{E}$ local ref.

Acquired: 11:3 Jul 14, 1997

Survey Co:
Rotation:

Filename: nn25a.all

Channels: Ch1 Ch2 Ch3 Ch4 Ch5 Ch6 Ch7

Platted: 08:39 Dec 08, 2000

< EMI - ElectroMagnetic Instruments 


\section{ROTATION ANGLE}

Battle Mtn.

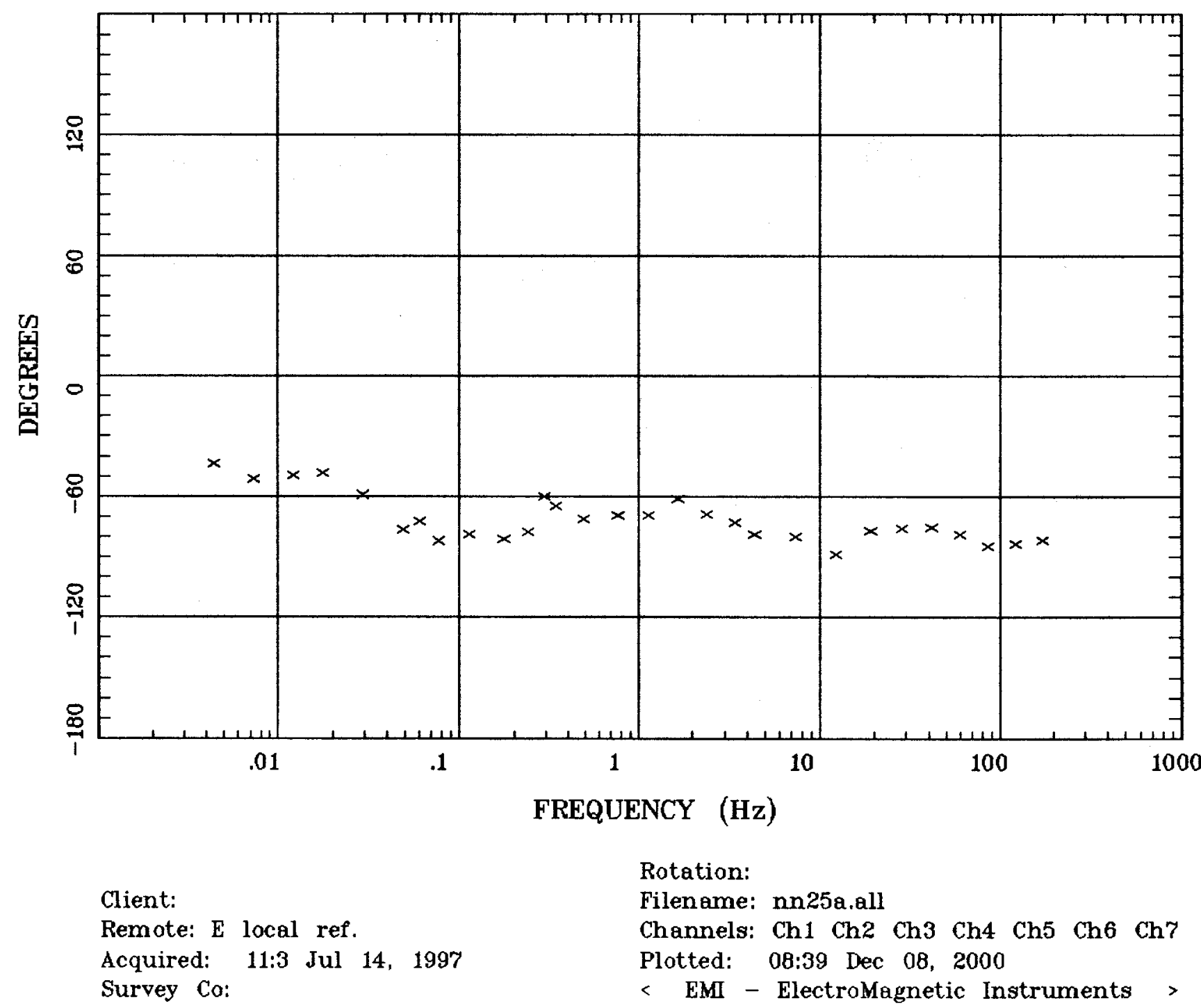




\section{Station 25A}

IMPEDANCE SKEW

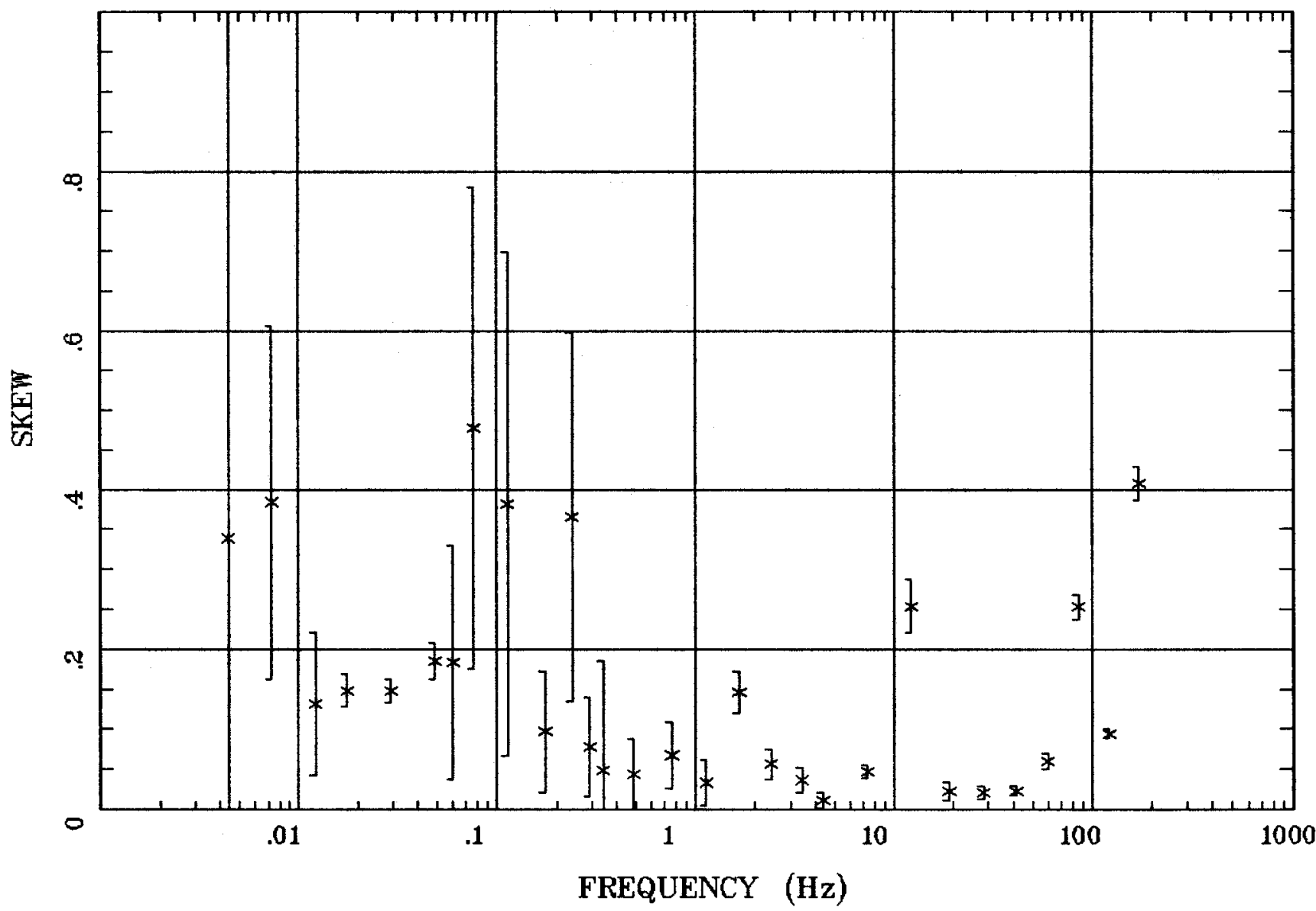

Client:

Remote: E local ref.

Acquired: 11:3 Jul 14, 1997

Survey Co:
Rotation:

Filename: nn25a.all

Channels: Ch1 Ch2 Ch3 ch4 ch5 Ch6 Ch7

Plotted: 08:39 Dec 08, 2000

< EMI - ElectroMagnetic Instruments > 


\section{Station 25A}

E MULT Coh.

Battle Mtn.

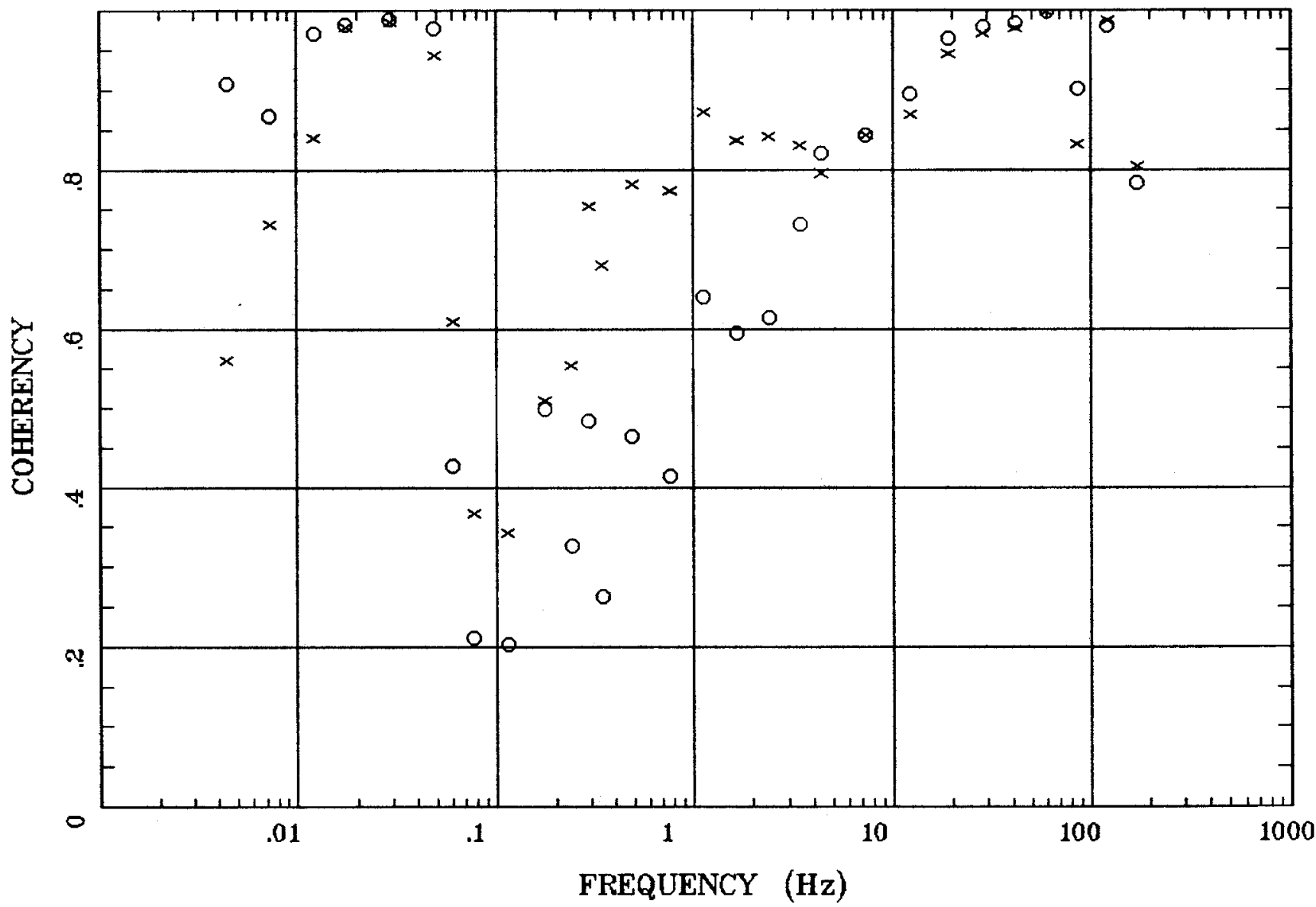

Client:

Remote: E local ref.

Acquired: 11:3 Jul 14, 1997

Survey Co:

Rotation

Filename: nn25a.all

Channels: Ch1 Ch2 Ch3 Ch4 Ch5 Ch6 Ch7

Plotted: 08:39 Dec 08, 2000

< EMI - ElectroMagnetic Instruments 
Battle Mtn.

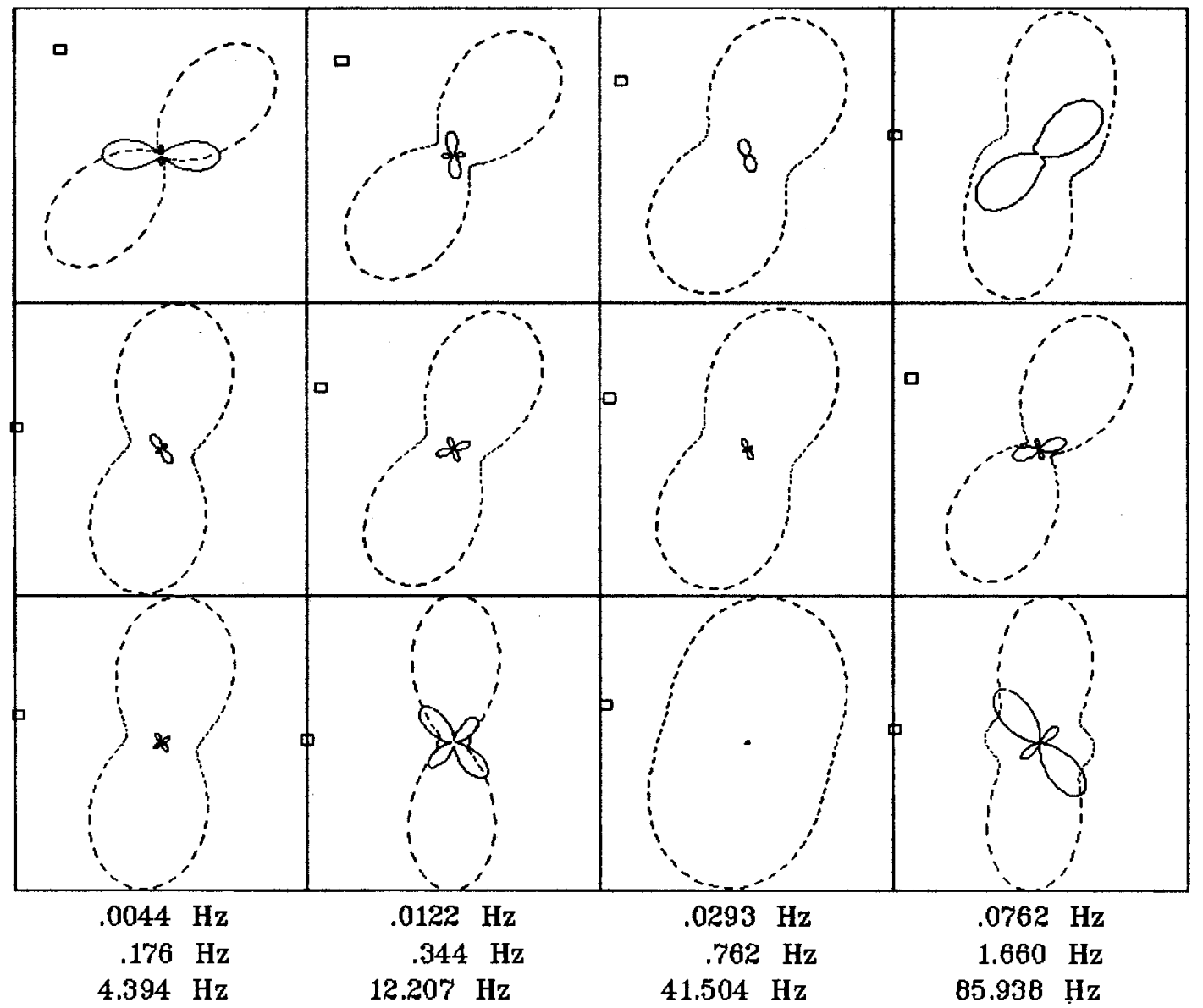

Client:

Remote: E local ref.

Acquired: 11:3 Jul 14, 1997

Survey Co:
Rotation:

Filename: nn25a.all

Channels: Ch1 Ch2 Ch3 Ch4 Ch5 Ch6 Ch7 Plotted: 08:39 Dec 08, 2000

< EMI - ElectroMagnetic Instruments > 


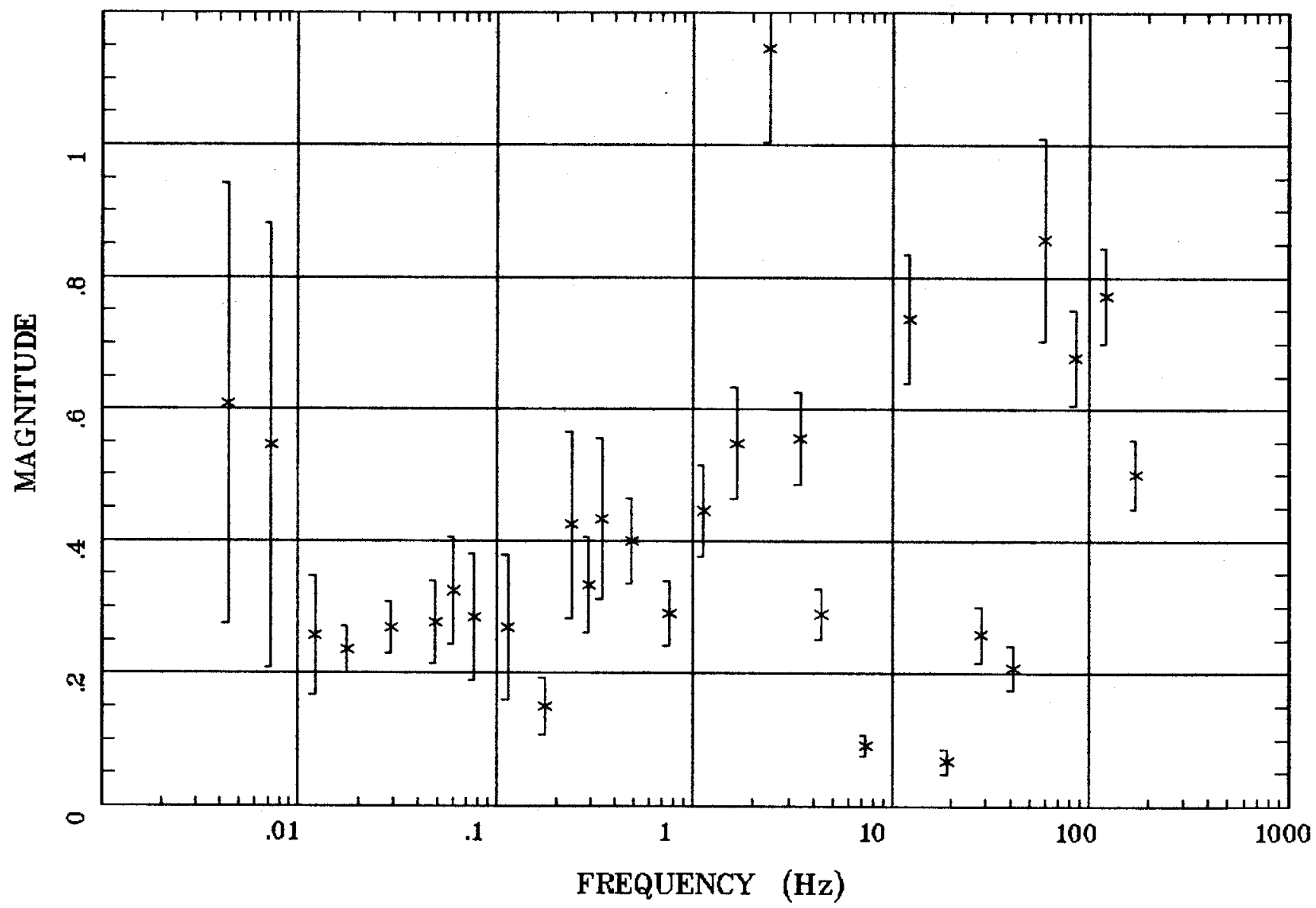

Client:

Remote: E local ref.

Acquired: 11:3 Jul 14, 1997

Survey Co:
Rotation:

Filename: nn25a.all

Channels: Ch1 Ch2 Ch3 Ch4 Ch5 Ch6 Ch7 Plotted: 08:39 Dec 08, 2000

$<$ EMI - ElectroMagnetic Instruments 


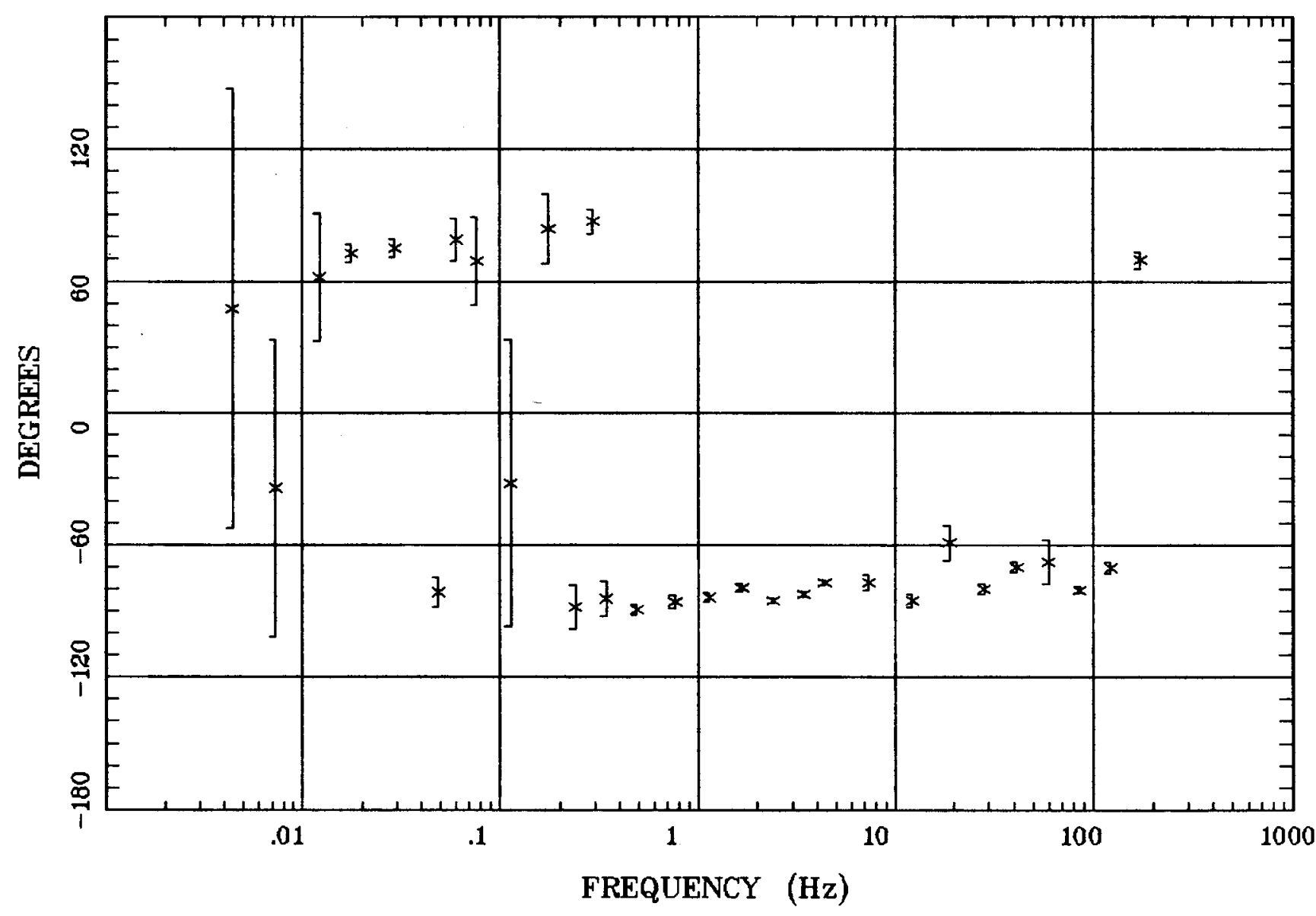

Client:

Remote: E local ref.

Acquired: 11:3 Jul 14, 1997

Survey Co:
Rotation:

Filename: nn25a.all

Channels: Ch1 Ch2 Ch3 Ch4 Ch5 Ch6 Ch7

Plotted: 08:39 Dec 08, 2000

$<$ EMI - ElectroMagnetic Instruments > 


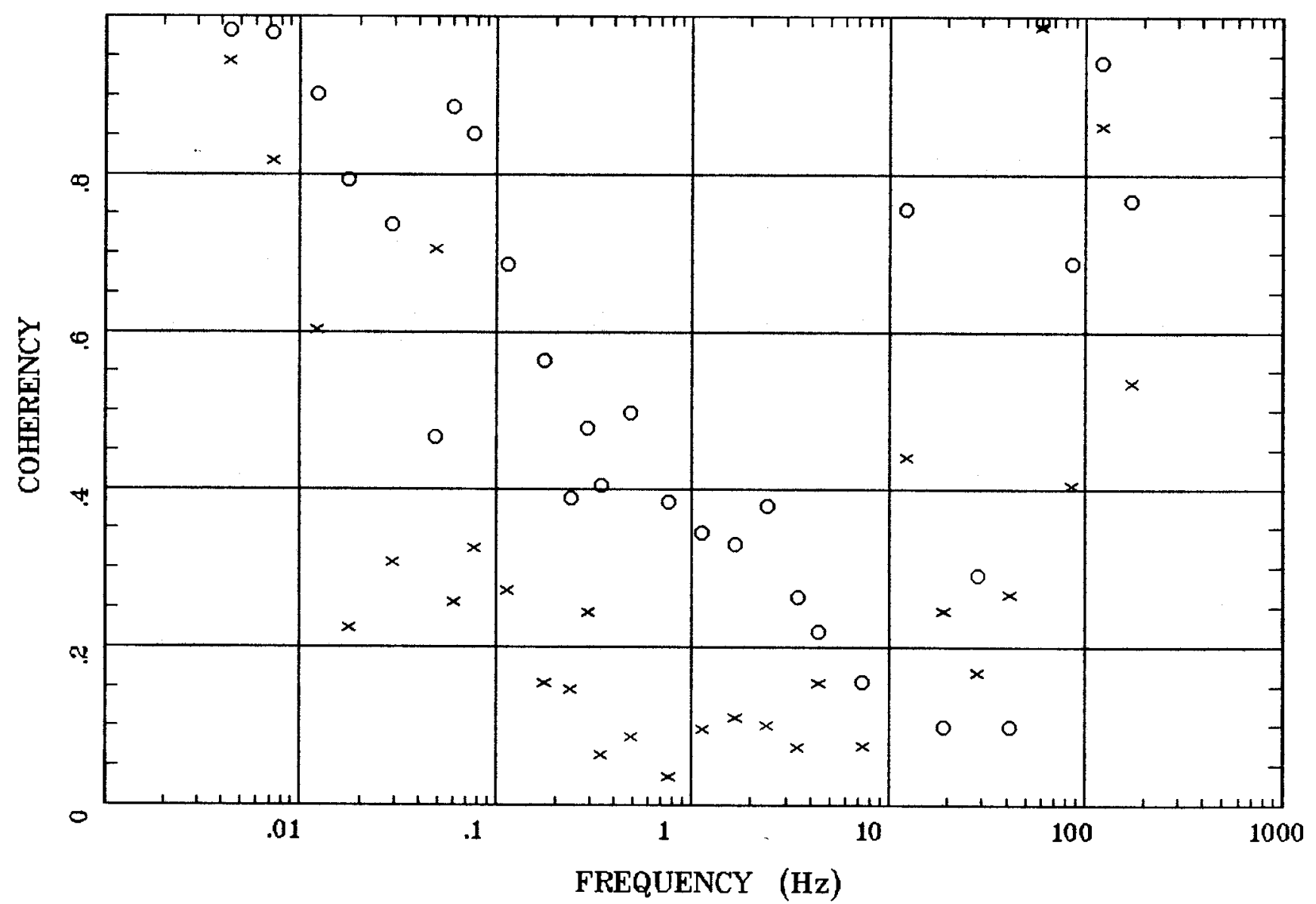

Client:

Remote: E local ref.

Acquired: 11:3 Jul 14, 1997 Survey Co:
Rotation:

Filename: nn25a.all

Channels: Ch1 Ch2 Ch3 Ch4 Ch5 Ch6 Ch7 Plotted: 08:39 Dec 08, 2000

< EMI - ElectroMagnetic Instruments 


\section{APPARENT RESISTIVITY}

Battle Mountain, NV

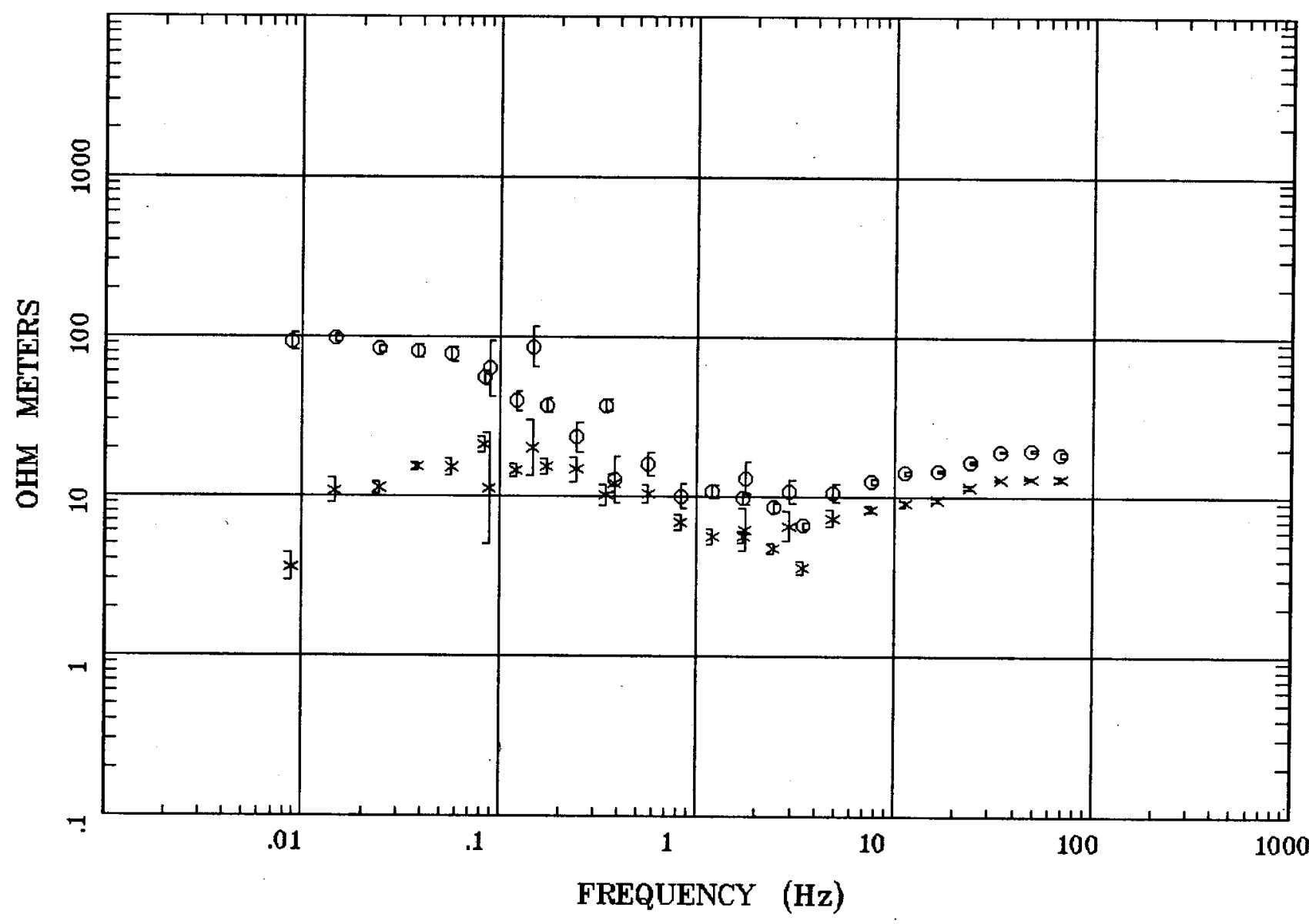

Client:

Remote: local

Acquired: 10:3 Jul 31, 1999

Survey Co:USGS
Rotation:

Filename: hr71.avg

Channels: Ch1 Ch2 Ch3 Ch4 Ch5 Ch3 Ch4

Plotted: 15:24 Jan 23, 2001

$<$ EMI - ElectroMagnetic Instruments 


\section{Station 71}

IMPEDANCE PHASE

Battle Mountain, NV

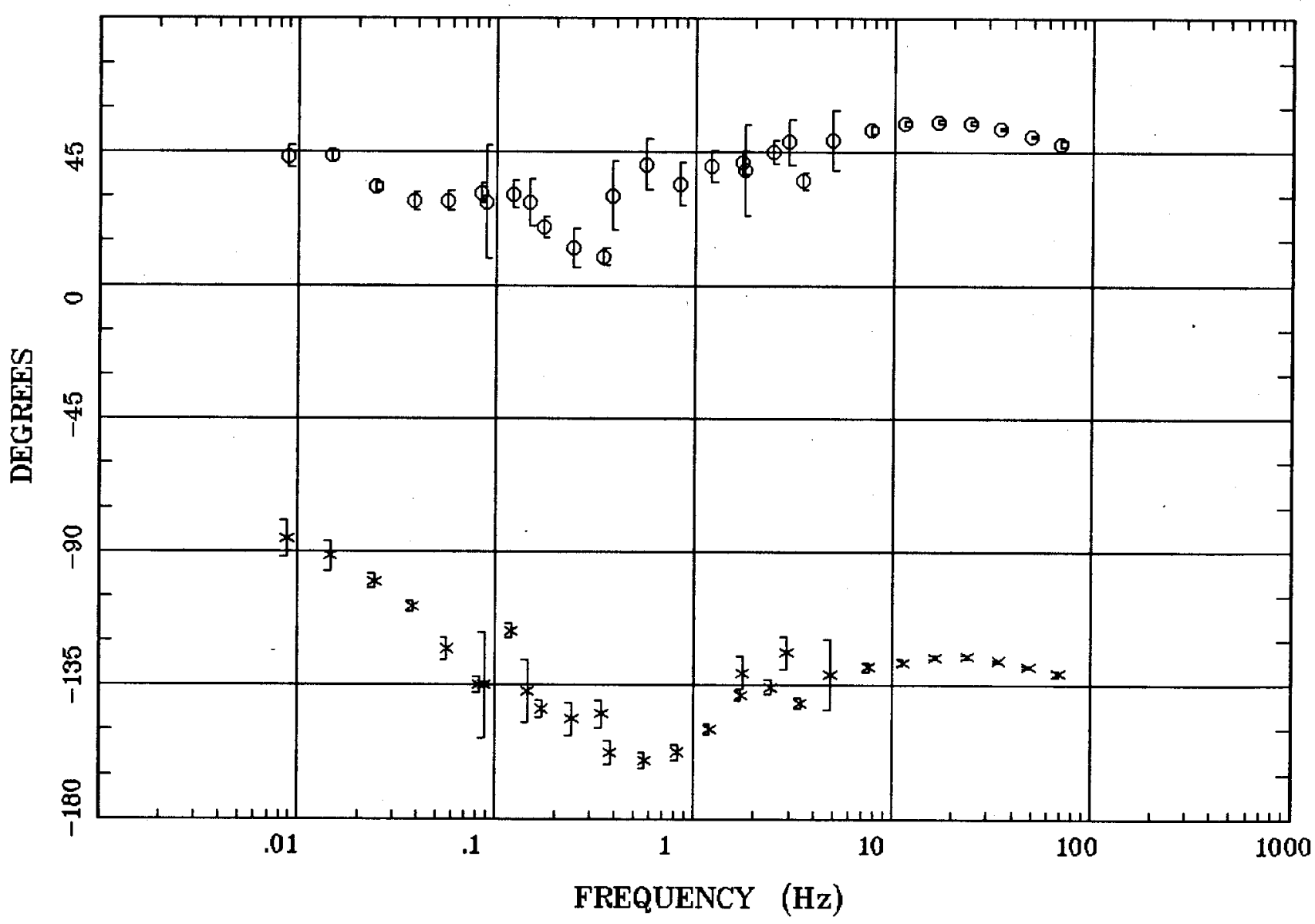

Client:

Remote: local

Acquired: 10:3 Jul 31, 1999

Survey Co:USGS
Rotation:

Filename: hr'71.avg

Channels: Ch1 Ch2 Ch3 Ch4 Ch5 Ch3 Ch4

Plotted: 15:24 Jan 23, 2001

< EMI - ElectroMagnetic Instruments > 


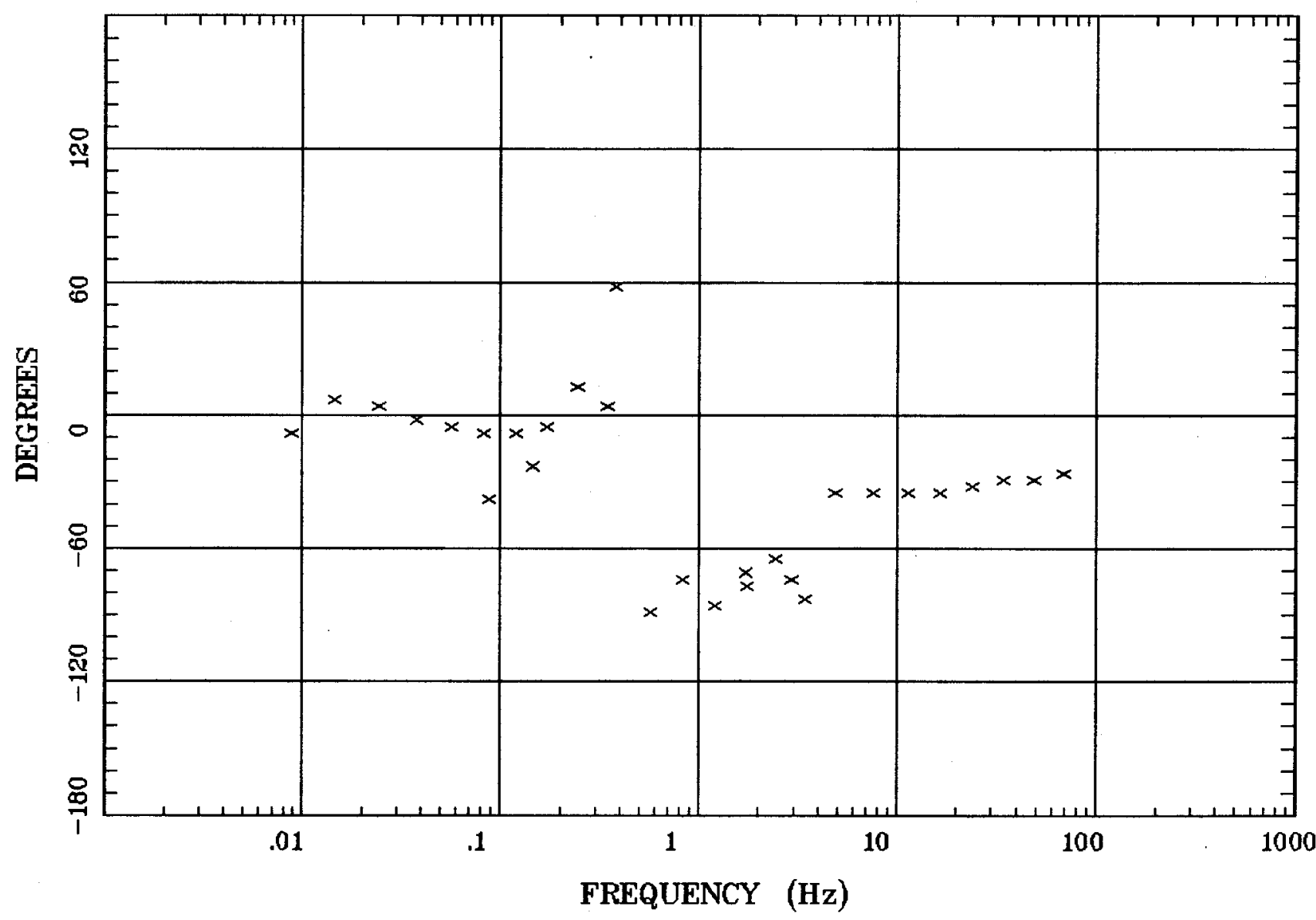

Client:

Remote: local

Acquired: 10:3 Jul 31, 1999

Survey Co:USGS
Rotation:

Filename: hr71.avg

Channels: Ch1 Ch2 Ch3 Ch4 Ch5 Ch3 Ch4 Plotted: 15:24 Jan 23, 2001

< EMI - ElectroMagnetic Instruments 


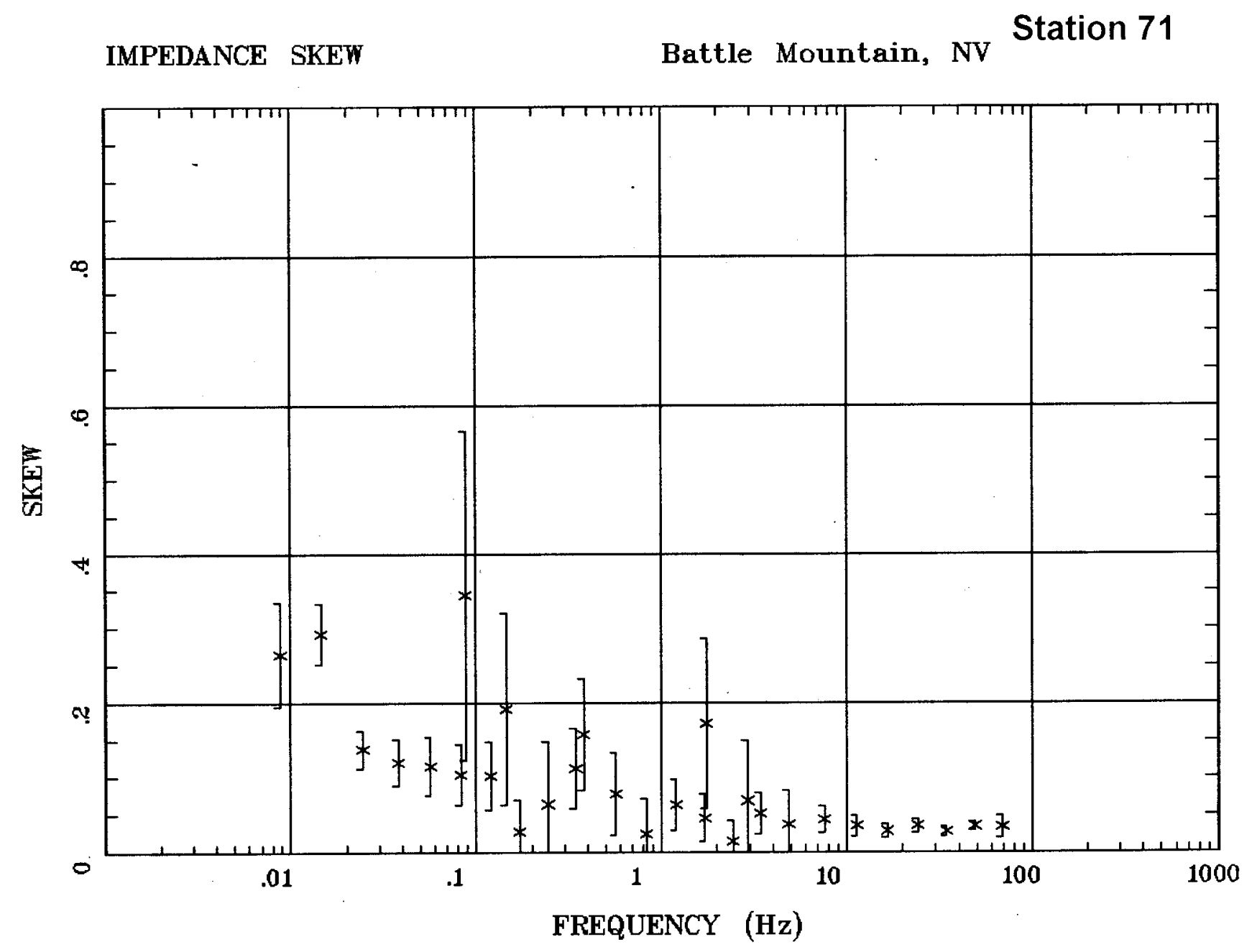

Client:

Remote: local Acquired: 10:3 Jul 31, 1999 Survey Co:USGS
Rotation:

Filename: hr71.avg

Channels: Ch1 Ch2 Ch3 Ch4 Ch5 Ch3 Ch4 Plotted: 15:24 Jan 23, 2001

< EMI - ElectroMagnetic Instruments > 


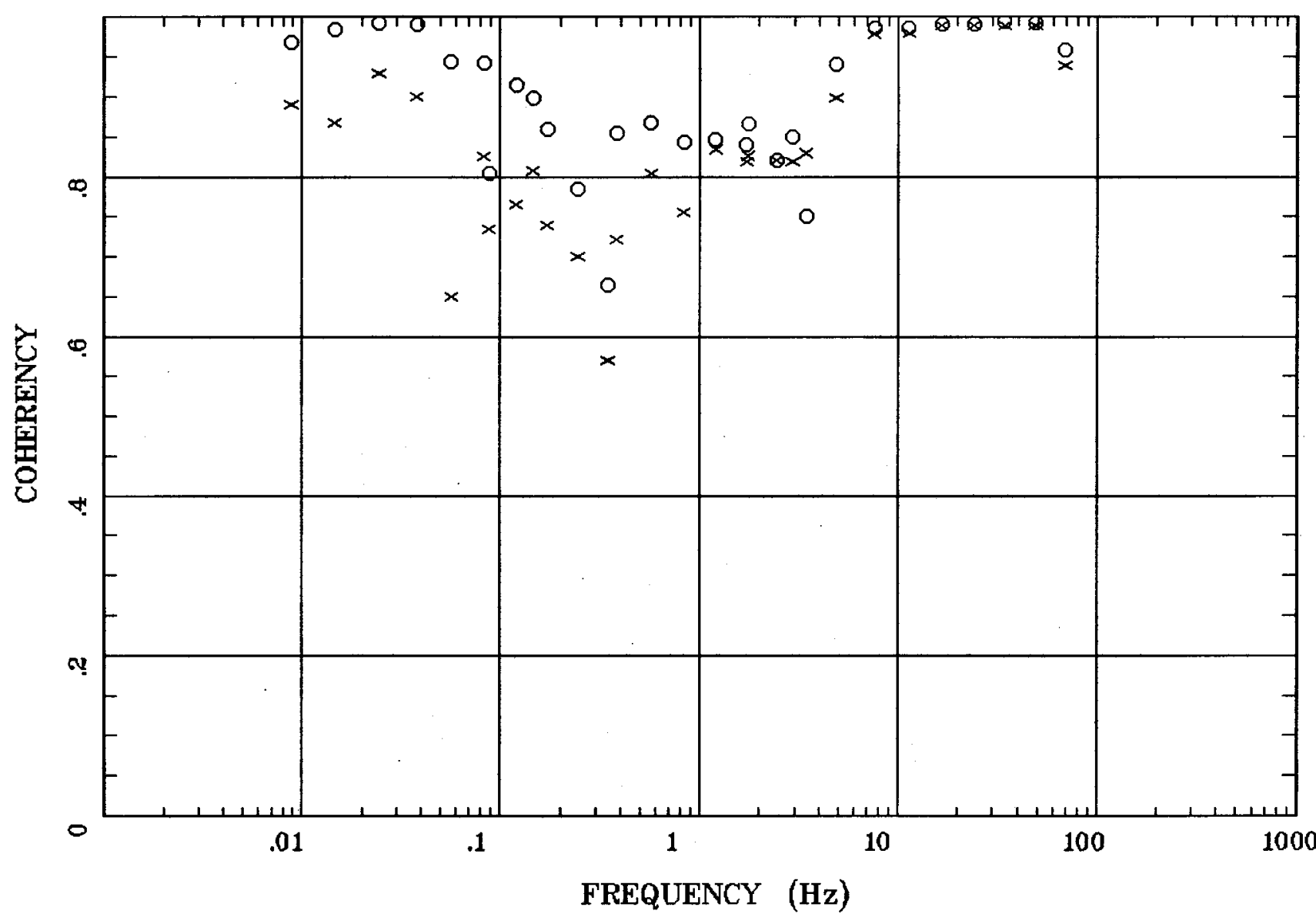

Client:

Remote: local

Acquired: $10: 3$ Jul 31, 1999

Survey Co:USGS
Rotation:

Filename: hr71.avg

Channels: Ch1 Ch2 Ch3 Ch4 Ch5 Ch3 Ch4

Plotted: 15:24 Jan 23, 2001

< EMI - ElectroMagnetic Instruments > 


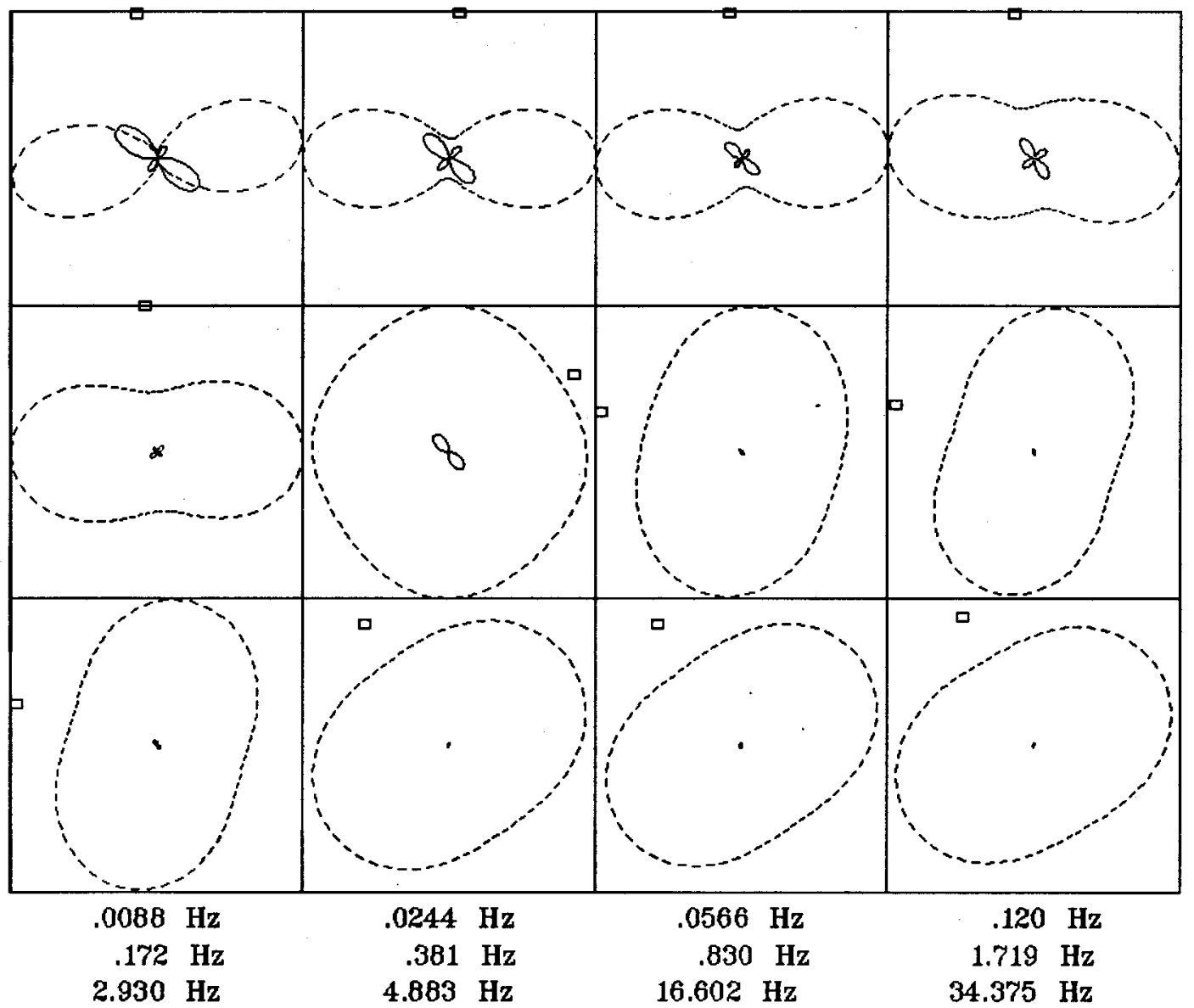

Client:

Remote: local

Acquired: 10:3 Jul 31, 1999 Survey Co:USGS
Rotation:

Filename: hr71.avg

Channels: Ch1 Ch2 Ch3 Ch4 Ch5 Ch3 Ch4 Plotted: 15:24 Jan 23, 2001

< EMI - ElectroMagnetic Instruments 


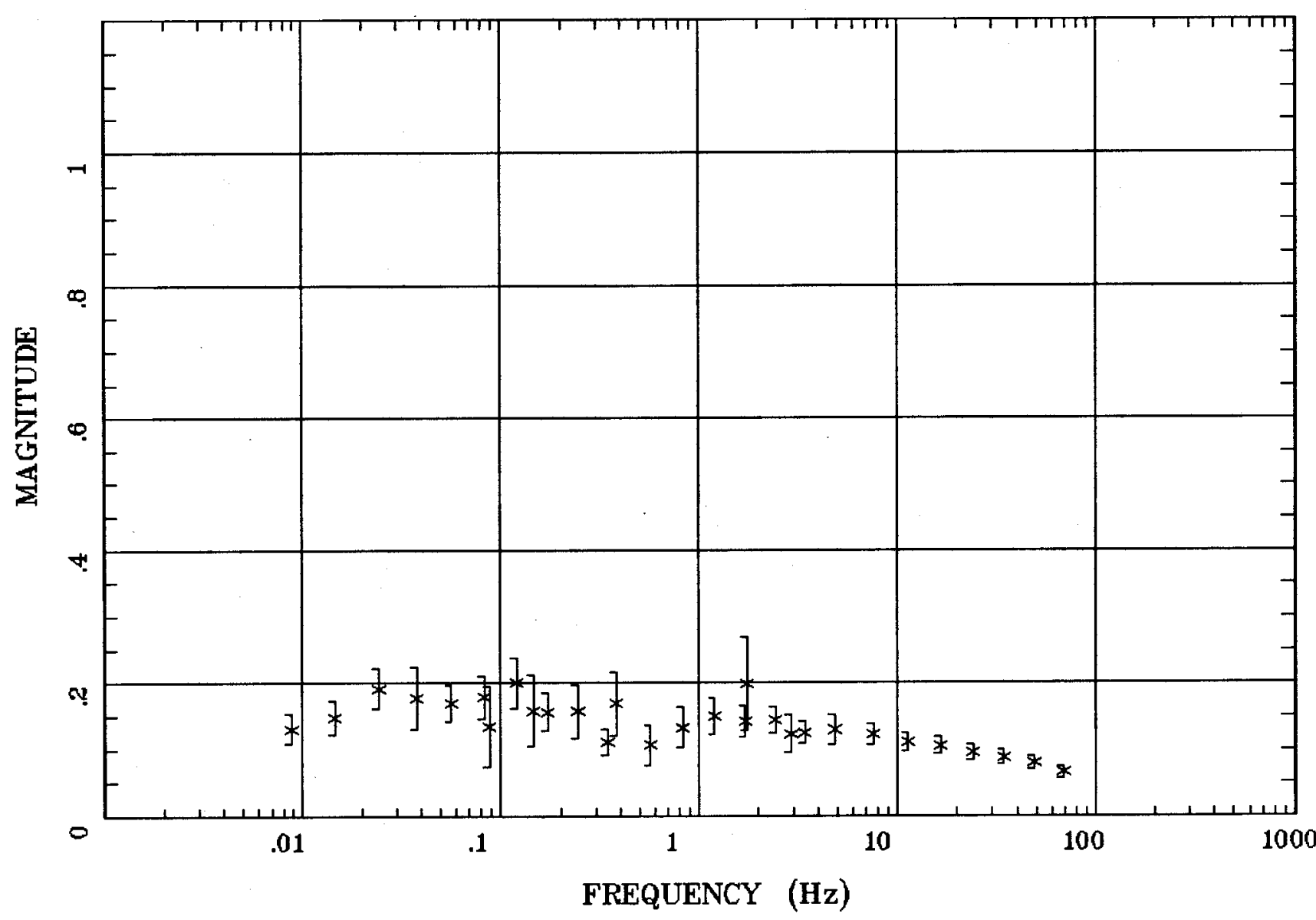

Client:

Remote: local

Acquired: 10:3 Jul 31, 1999

Survey Ca:USGS
Rotation:

Filename: hr71.avg

Channels: Ch1 Ch2 Ch3 ch4 Ch5 Ch3 Ch4 Plotted: 15:25 Jan 23, 2001

< EMI - ElectroMagnetic Instruments 
TIPPER STRIKE

Battle Mountain, NV

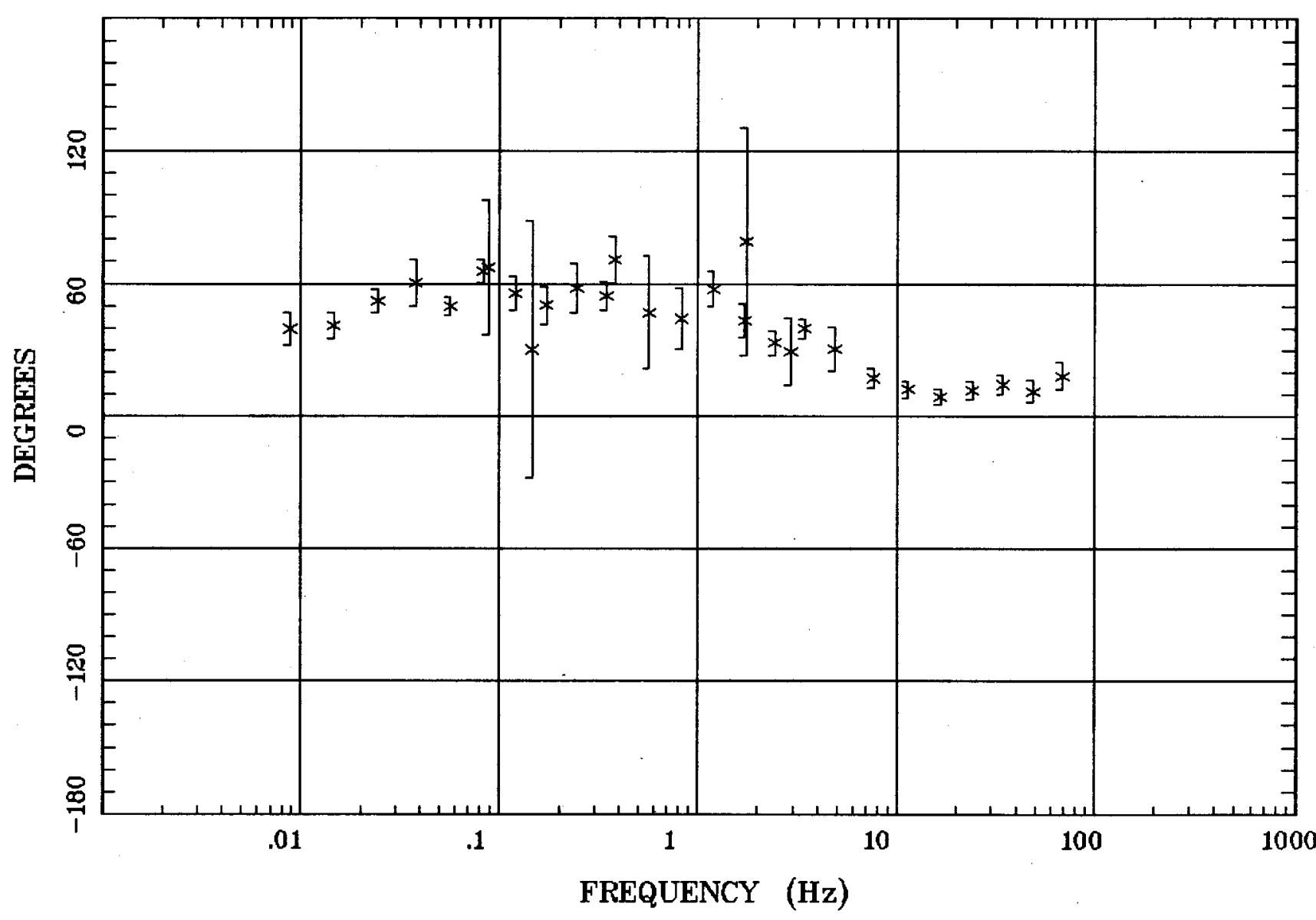

Client:

Remote: local

Acquired: 10:3 Jul 31, 1999 Survey Co:USGS

\section{Rotation:}

Filename: hrr1.avg

Channels: Ch1 Ch2 Ch3 Ch4 Ch5 Ch3 Ch4 Plotted: 15:25 Jan 23, 2001

< EMI - ElectroMagnetic Instruments 


\section{Station 71}

HzHx.x Coh HzHy.o

Battle Mountain, NV

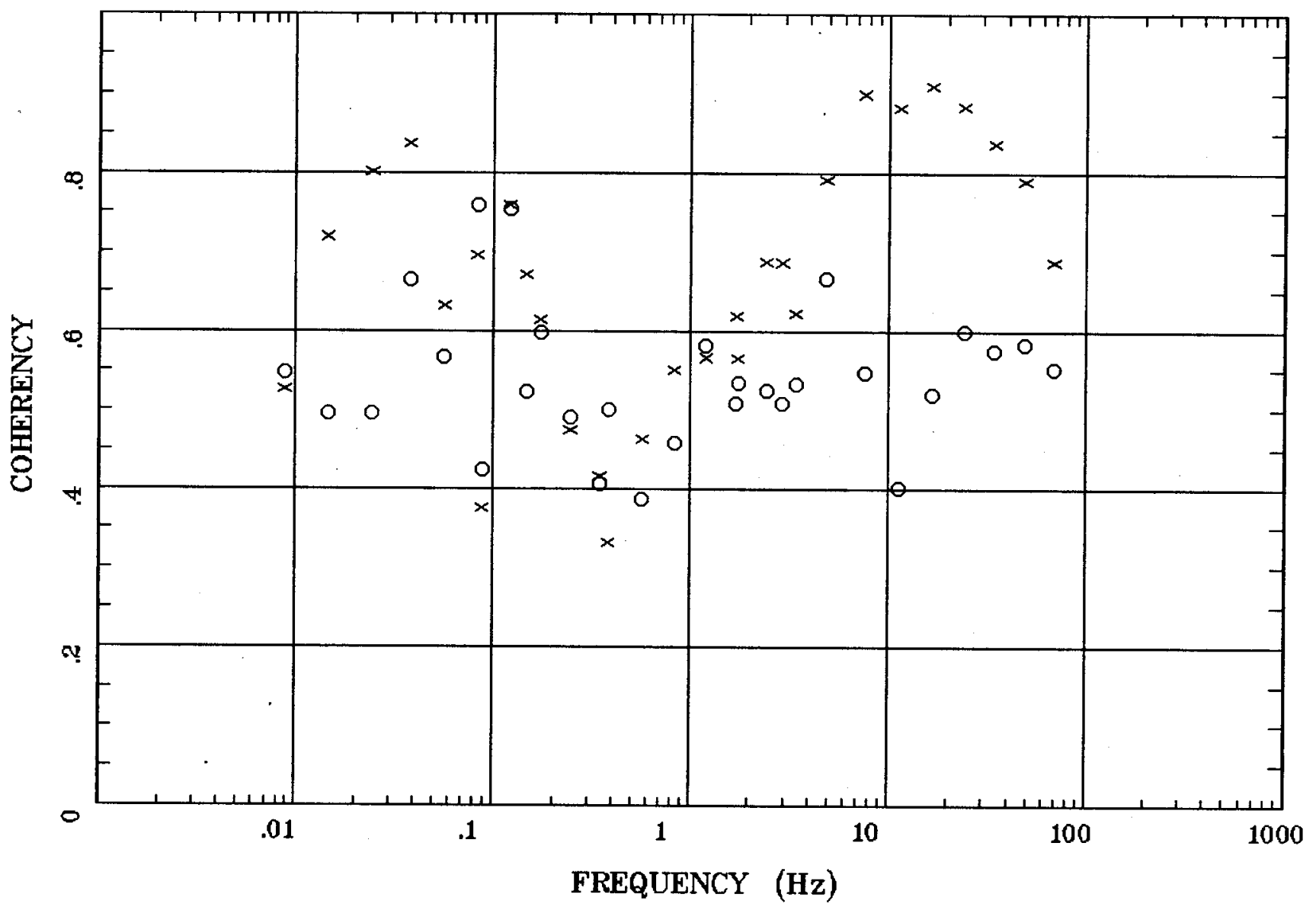

Client:

Remote: local

Acquired: 10:3 Jul 31, 1999

Survey Co:USGS
Rotation:

Filename: hr71.avg

Channels: Ch1 Ch2 Ch3 Ch4 Ch5 Ch3 Ch4

Plotted: 15:25 Jan 23, 2001

$<$ EMI - ElectroMagnetic Instruments 
APPARENT RESISTIVITY

Battle Mtn.

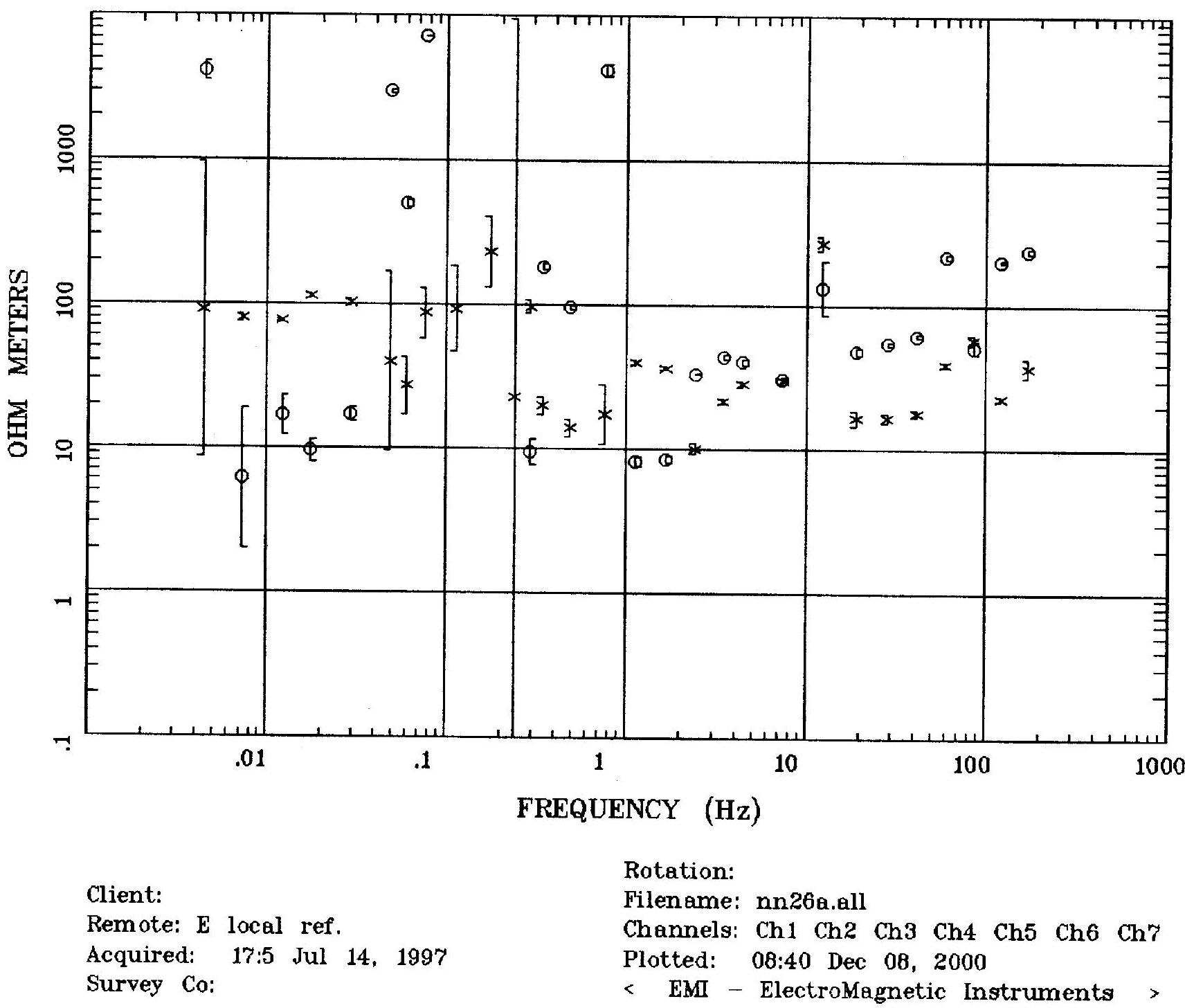




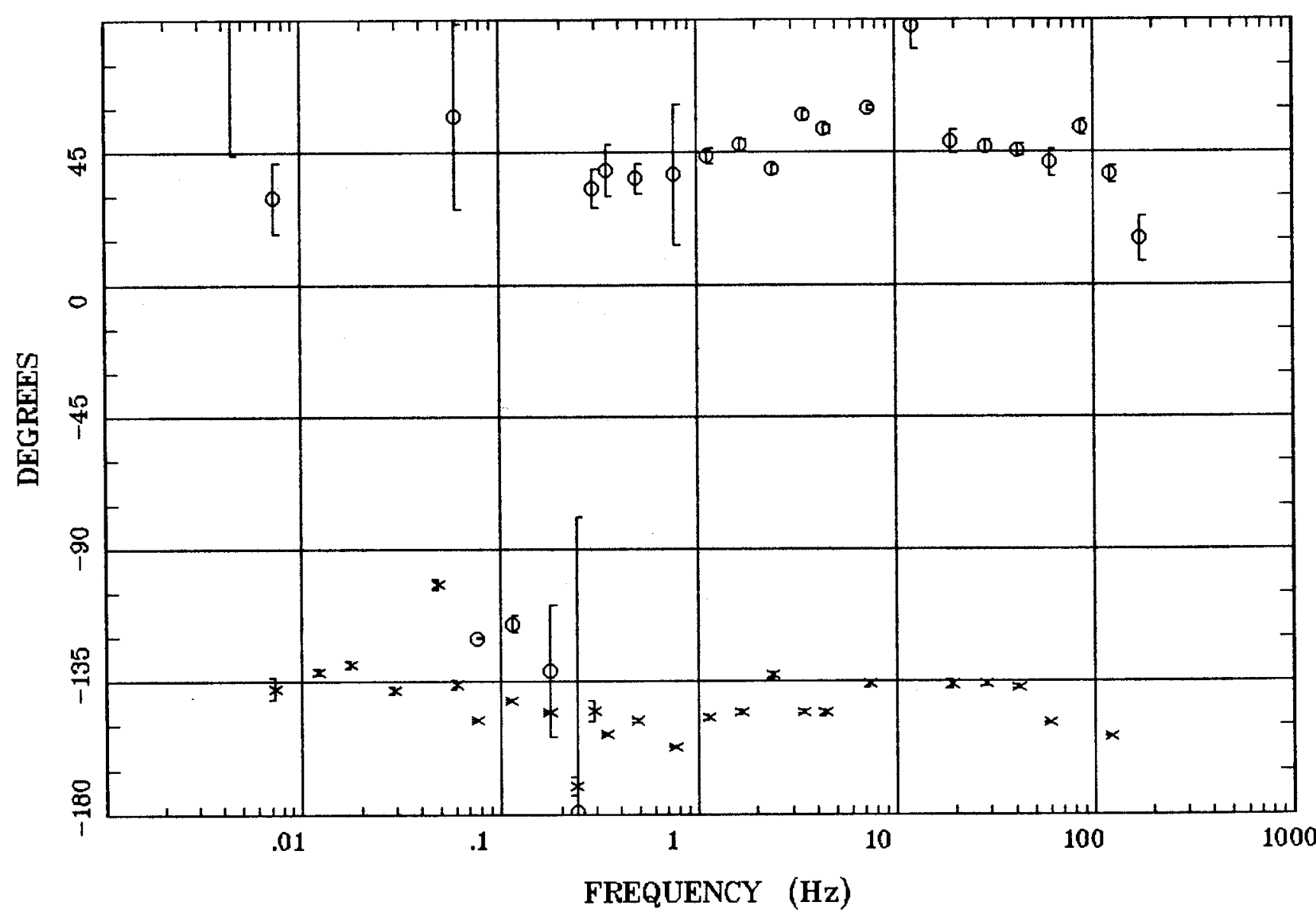

Client:

Remote: E local ref.

Acquired: 17:5 Jul 14, 1997 Survey Co:
Rotation:

Filename: nn26a.all

Channels: Ch1 Ch2 Ch3 Ch4 Ch5 Ch6 Ch7 Plotted: 08:40 Dec 08, 2000

< EMI - ElectroMagnetic Instruments 
ROTATION ANGLE

Battle Mtn.

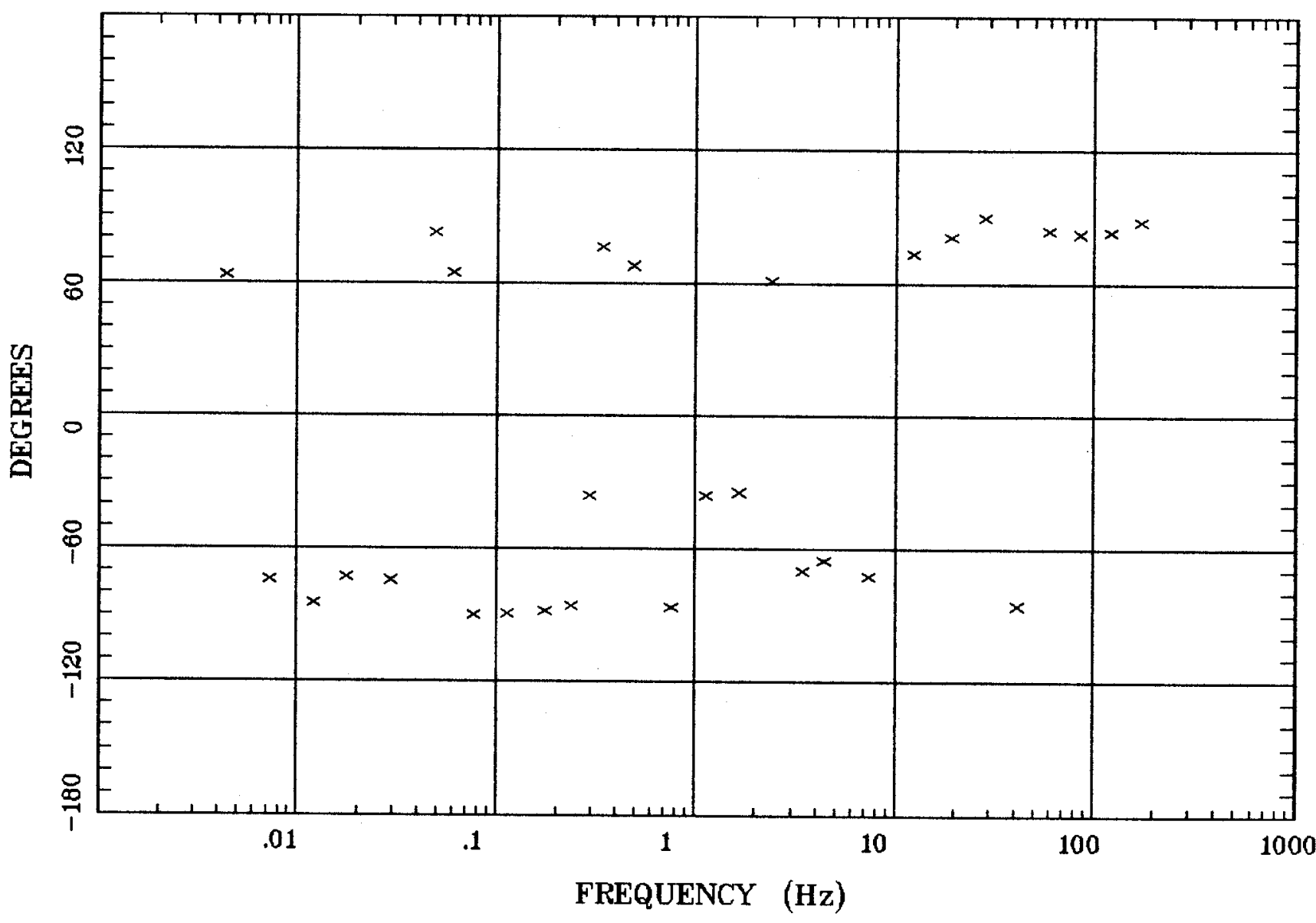

Client:

Remote: E local ref.

Acquired: 17:5 Jul 14, 1997

Survey Co:
Rotation:

Filename: nn26a.all

Channels: Ch1 Ch2 Ch3 Ch4 Ch5 Ch6 Ch7

Plotted: 08:40 Dec 08, 2000

<EMI - ElectroMagnetic Instruments > 


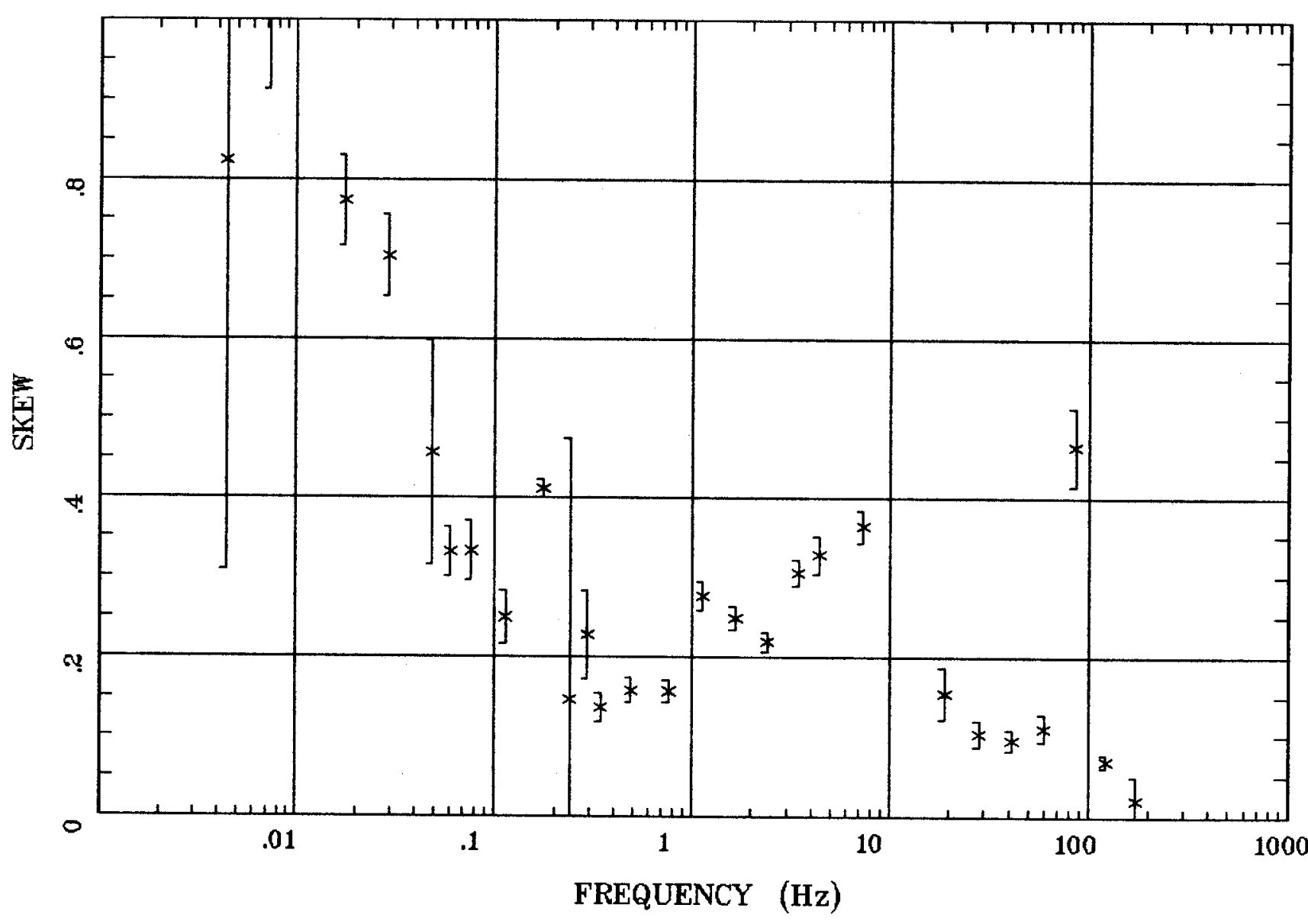

Client:

Remote: E local ref.

Acquired: $17: 5 \mathrm{Jul}$ 14, 1997

Survey Co:
Rotation:

Filename: nn26a.all

Channels: Ch1 Ch2 Ch3 Ch4 Ch5 Ch6 Ch7

Plotted: 08:40 Dec 08, 2000

$<$ EMI - ElectroMagnetic Instruments 
Station 26A

E MULT Coh.

Battle Mtn.

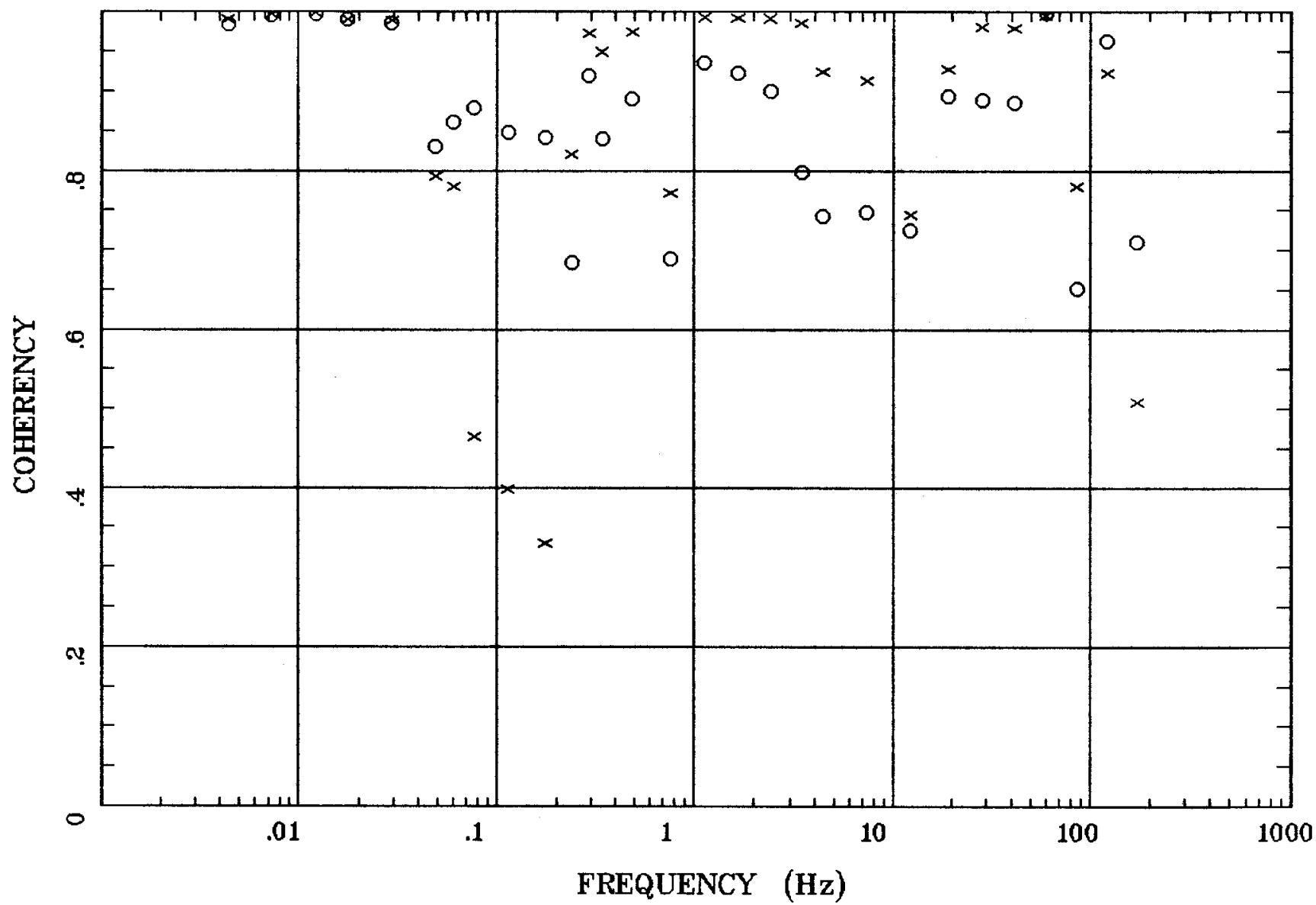

Client:

Remote: E local ref.

Acquired: 17:5 Jul 14, 1997

Survey Co:
Ratation:

Filename: nn26a.all

Channels: Ch1 Ch2 Ch3 Ch4 Ch5 Ch6 Ch7

Plotted: 08:40 Dec 08, 2000

< EMI - ElectroMagnetic Instruments > 


\section{POLAR PLOTS}

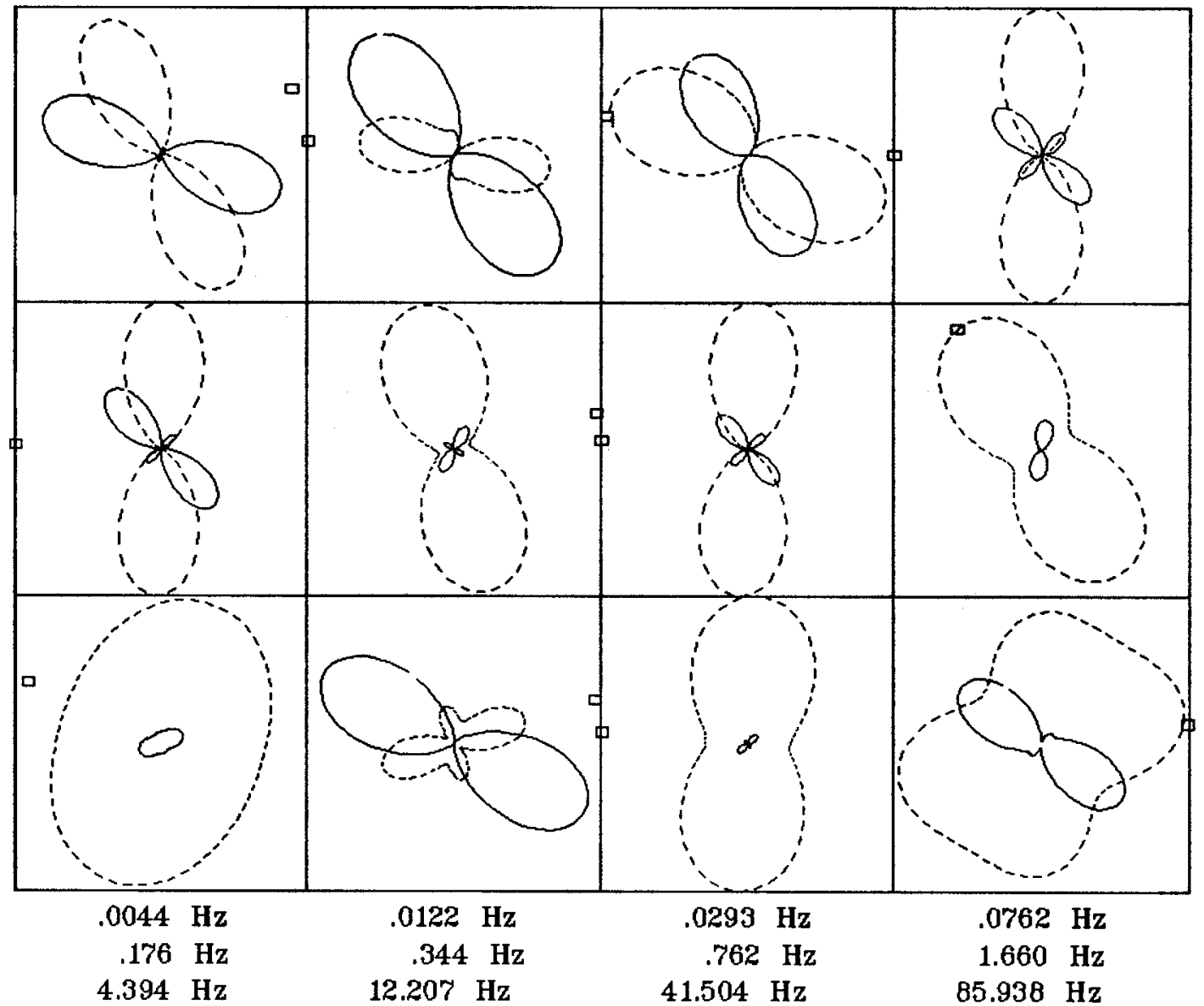

Client:

Remote: E local ref.

Acquired: 17:5 Jul 14, 1997

Survey $\mathrm{Co}$
Battle Mtn.

Rotation:

Filename: nn26a.all

Channels: Ch1 Ch2 Ch3 Ch4 Ch5 Ch6 Ch7

Plotted: 08:40 Dec 08, 2000

$<$ EMI - ElectroMagnetic Instruments > 
TIPPER MAGNITUDE

Battle Mtn.

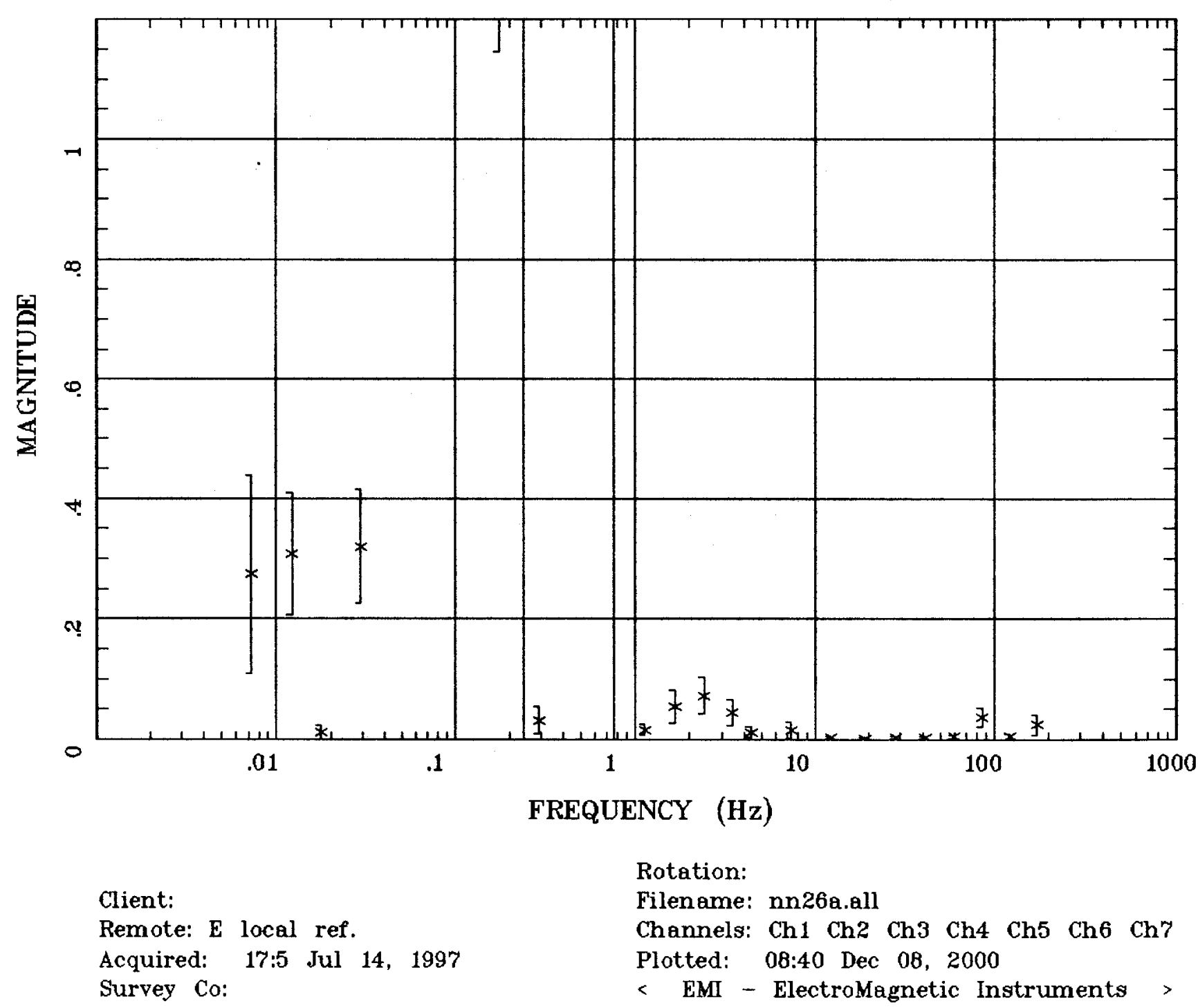


TIPPER STRIKE

Battle Mtn.

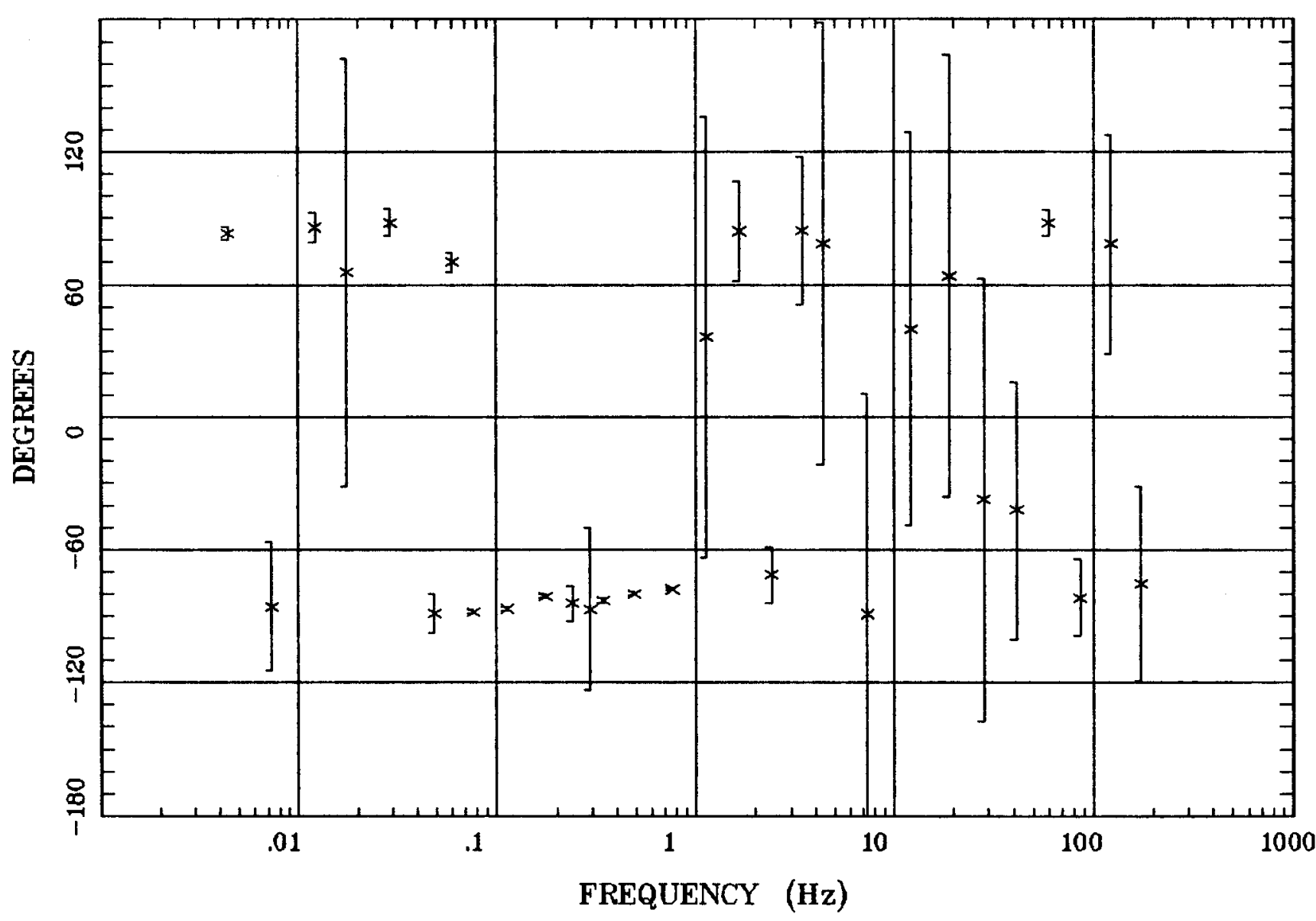

Client:

Remote: E local ref.

Acquired: 17:5 Jul 14, 1997

Survey Co:
Rotation:

Filename: nn26a.all

Channels: Ch1 Ch2 Ch3 Ch4 Ch5 Ch6 Ch7

Plotted: 08:40 Dec 08, 2000

< EMI - ElectroMagnetic Instruments > 


\section{Station 26A}

HzHx.x Coh HzHy.o

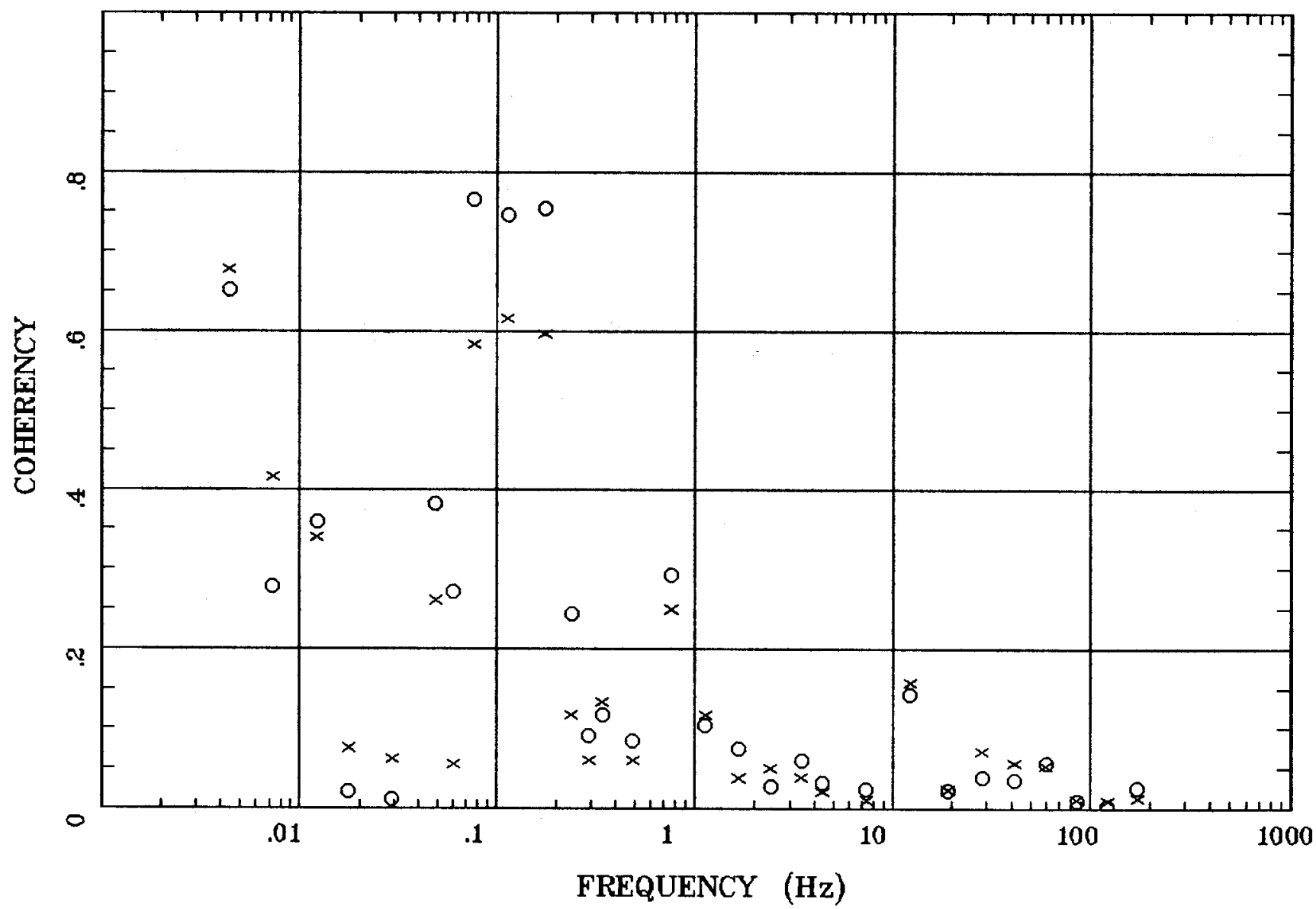

Client:

Remote: E local ref.

Acquired: $17: 5$ Jul 14, 1997 Survey Ca:
Rotation:

Filename: nn26a.all

Channels: Ch1 Ch2 Ch3 Ch4 Ch5 Ch6 Chr Plotted: 08:40 Dec 08, 2000

< EMI - ElectroMagnetic Instruments > 
Station 27A

APPARENT RESISTIVITY _...Battle Mtn...

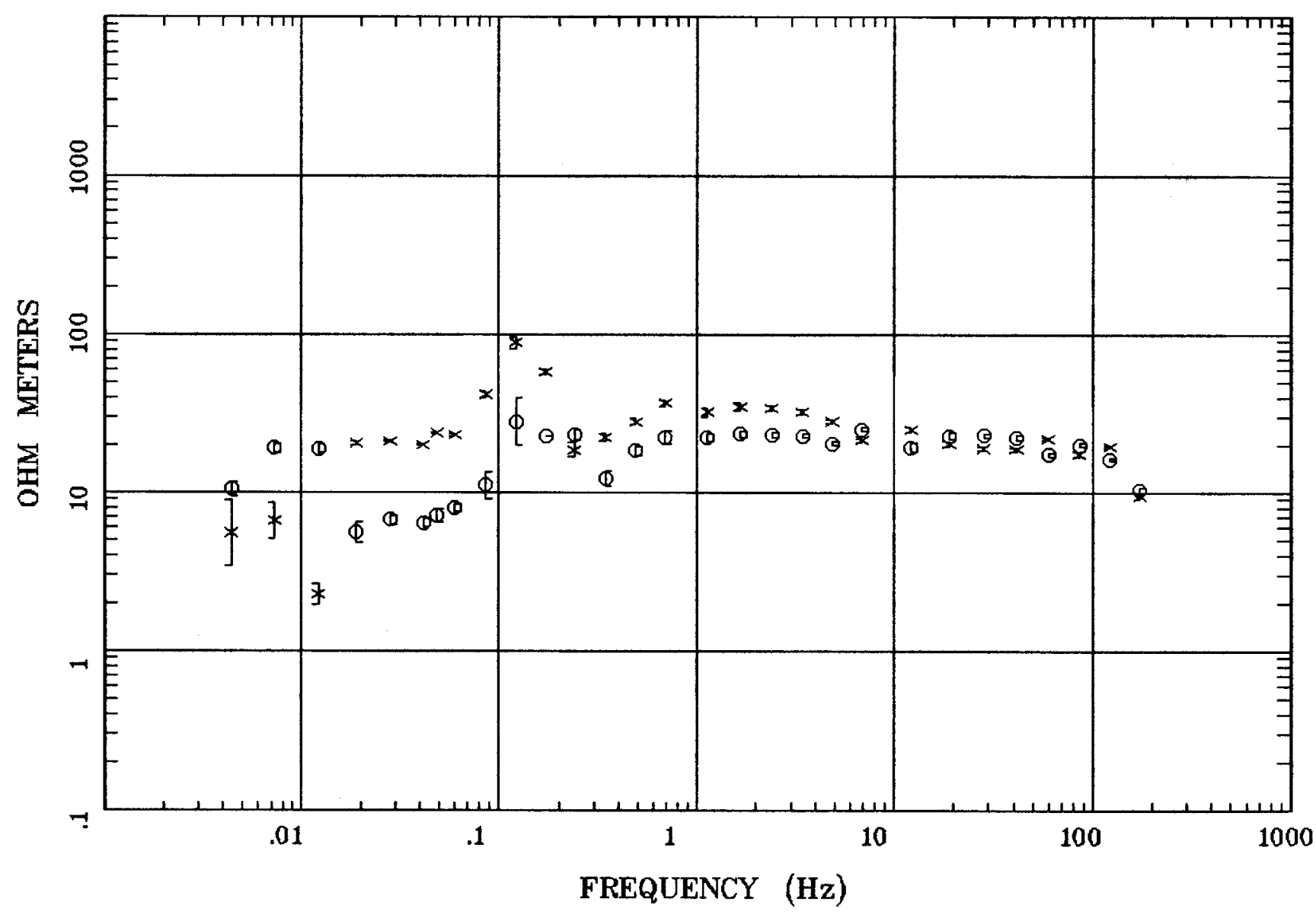

Client:

Remote: $\mathrm{E}$ local ref.

Acquired: 12:0 Jul 21, 1997 Survey Co:
Rotation:

Filename: nn27a.all

Channels: Ch6 Ch7 Ch8 Ch9 Ch10Ch1 Ch2 Plotted: 08:43 Dec 08, 2000

$<$ EMI - ElectroMagnetic Instruments 
IMPEDANCE PHASE

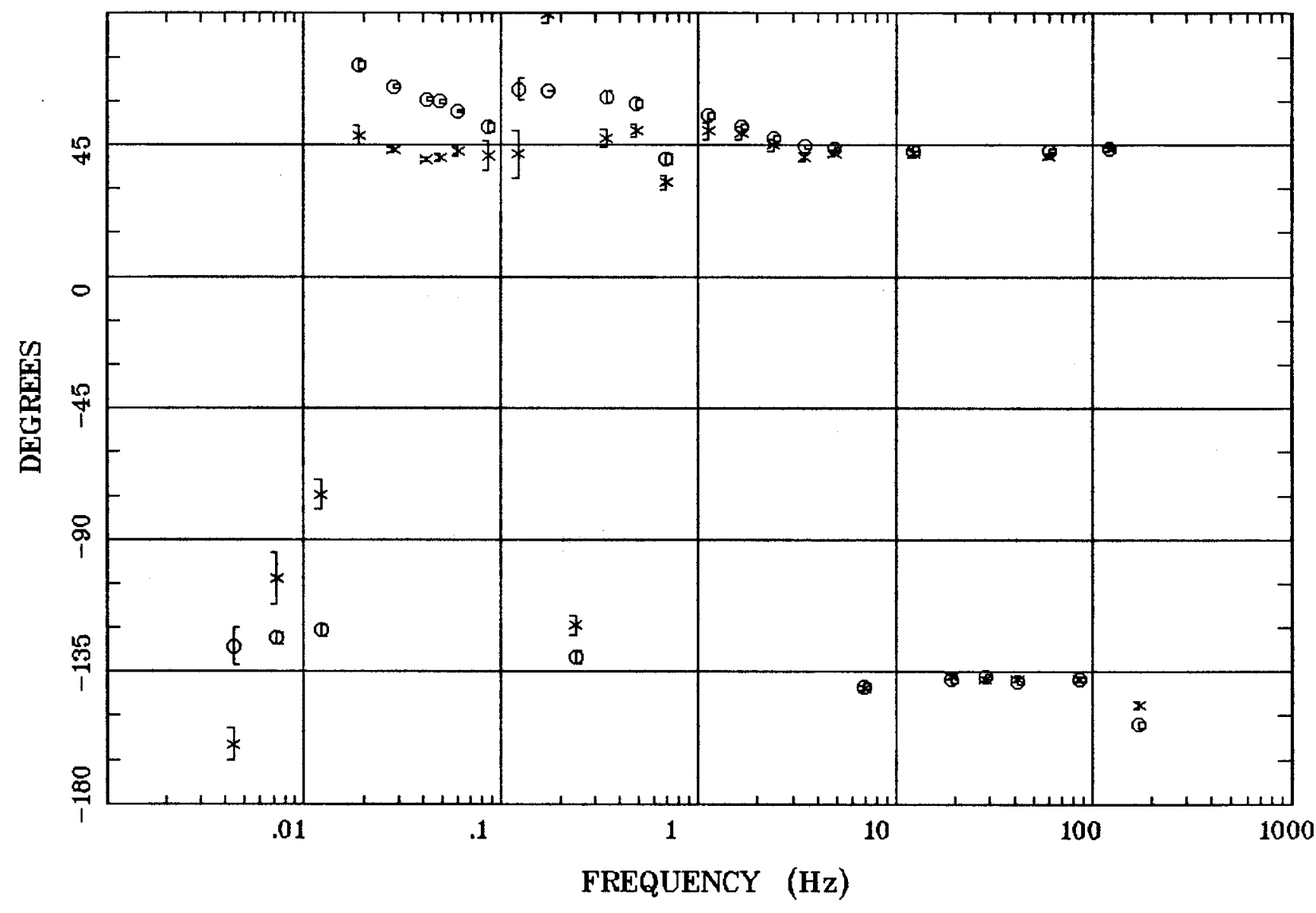

Client:

Remote: E local ref.

Acquired: 12:0 Jul 21, 1997

Survey Co:
Rotation:

Filename: nn27a.all

Channels: Ch6 Chr7 Ch8 Ch9 Ch10Ch1 Ch2

Plotted: 08:43 Dec 08, 2000

$<$ EMI - ElectroMagnetic Instruments > 
ROTATION ANGLE

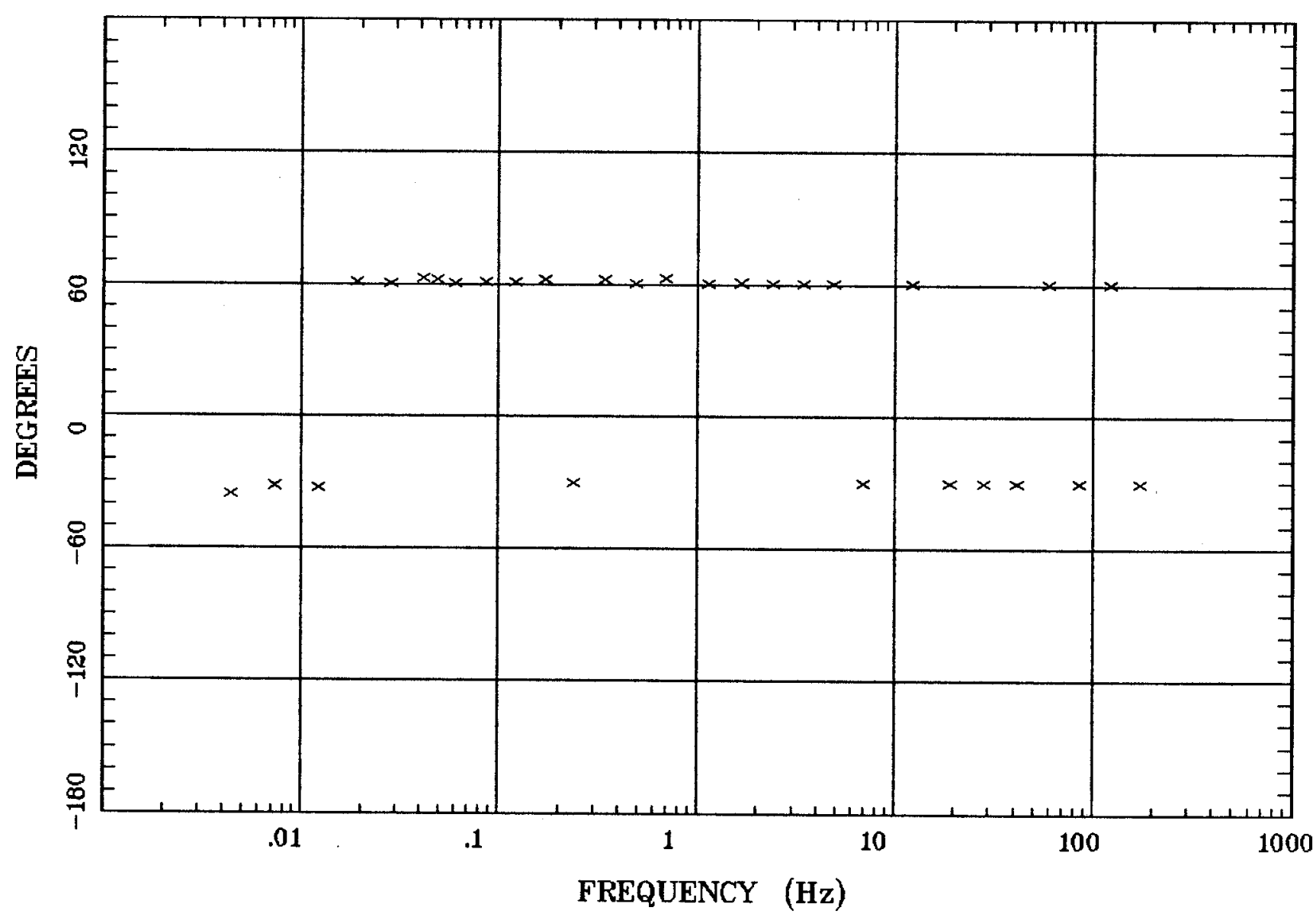

Client:

Remote: E local ref.

Acquired: 12:0 Jul 21, 1997 Survey Co:
Rotation:

Filename: nnzra.all

Channels: Ch6 Chr Ch8 Ch9 Ch10Ch1 Ch2

Plotted: 08:43 Dec 08, 2000

< EMI - ElectroMagnetic Instruments 
IMPEDANCE SKEW

...Battle Mtn...

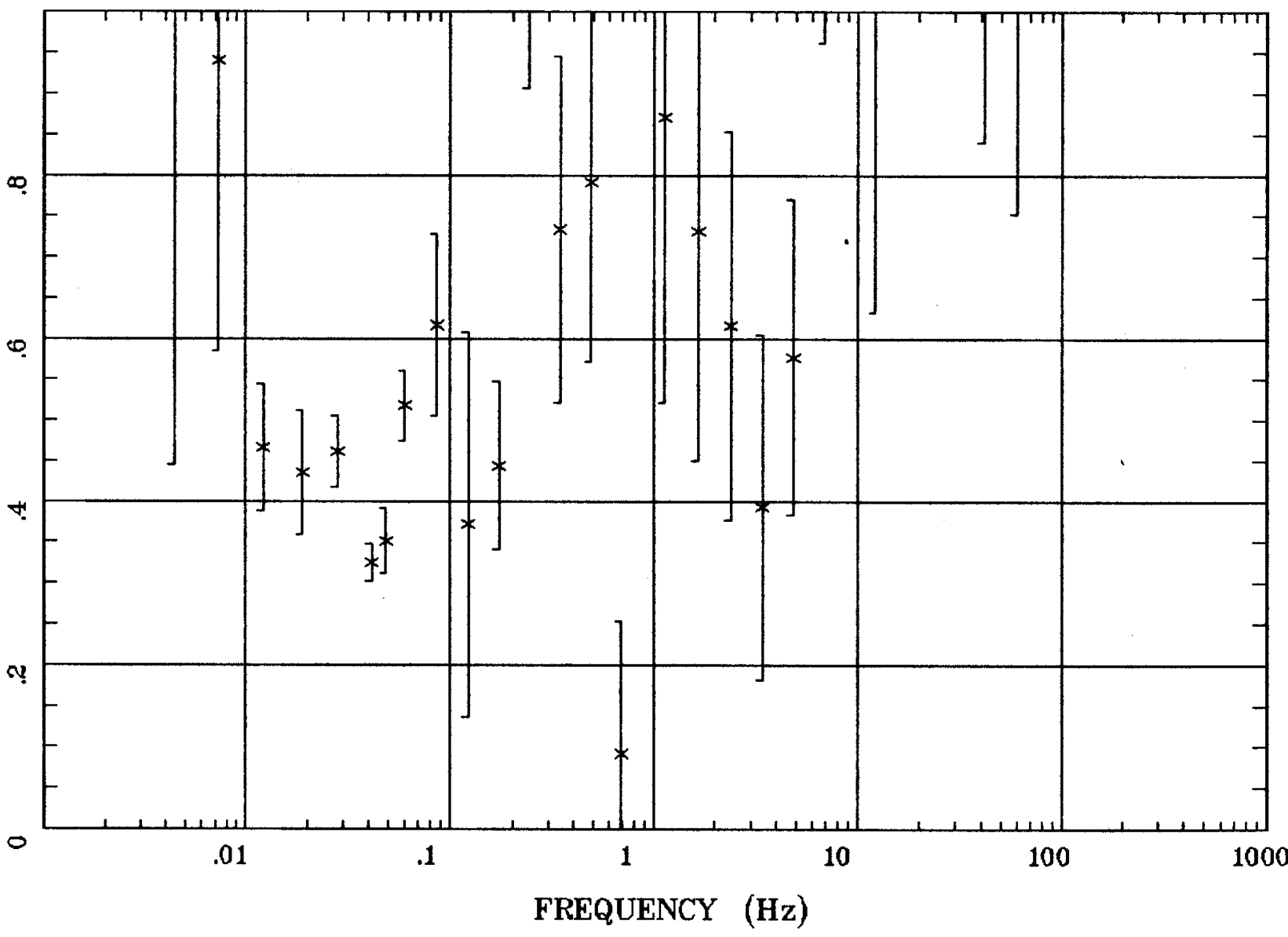

Client:

Remote: $\mathrm{E}$ local ref.

Acquired: 12:0 Jul 21, 1997 Survey Co:
Rotation:

Filename: nnz7a.all

Channels: Ch6 Ch7 Ch8 Ch9 Ch10Ch1 Ch2 Plotted: 08:43 Dec 08, 2000

< EMI - ElectroMagnetic Instruments > 


\section{Station 27A}

E MULT Coh. $\quad$...Battle Mtn...

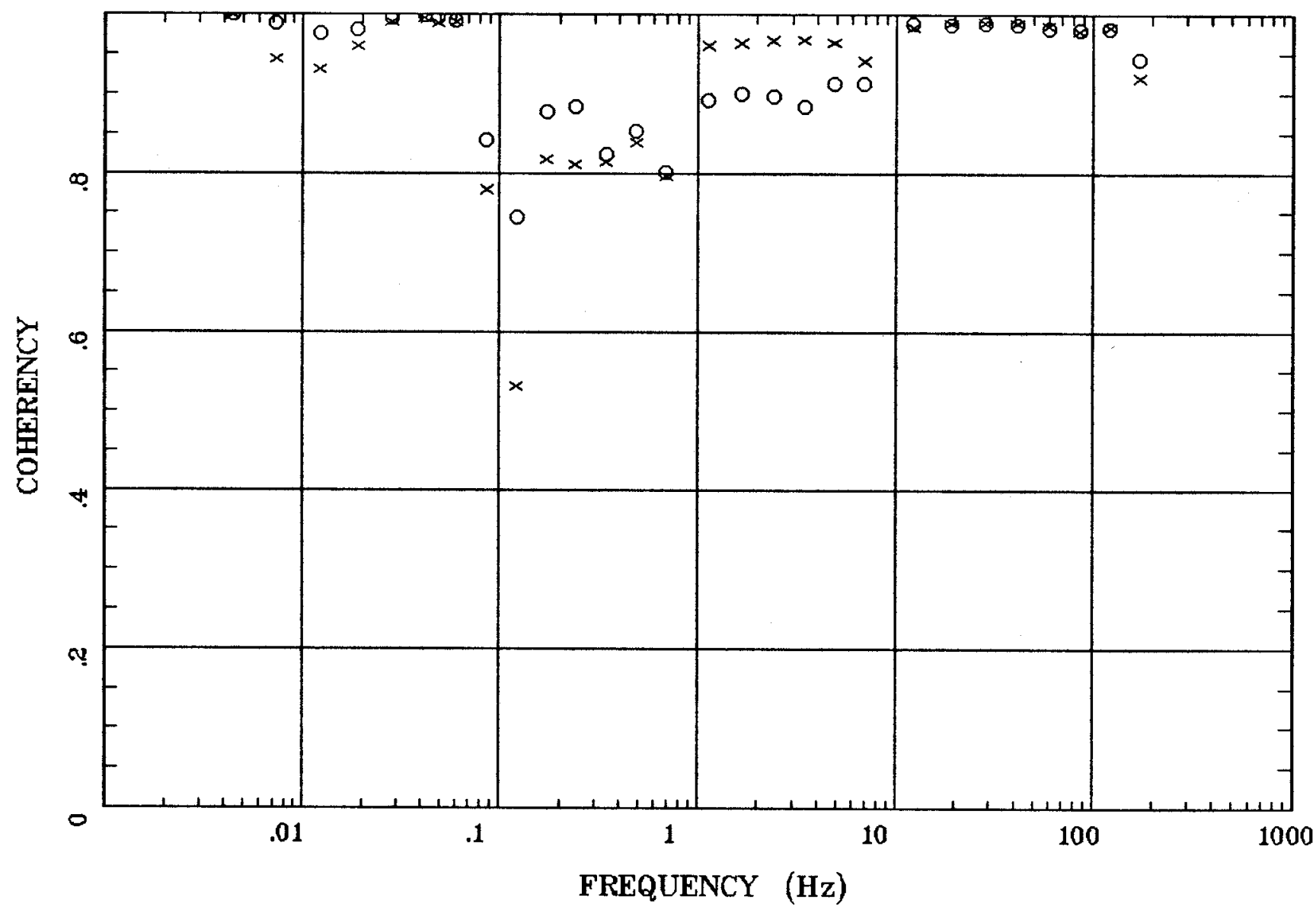

Client:

Remote: E local ref.

Acquired: 12:0 Jul 21, 1997

Survey Co:
Rotation:

Filename: nn27a.all

Channels: Ch6 Chr Ch8 Ch9 Ch10Ch1 Ch2

Plotted: 08:43 Dec 08, 2000

$<$ EMI - ElectroMagnetic Instruments 


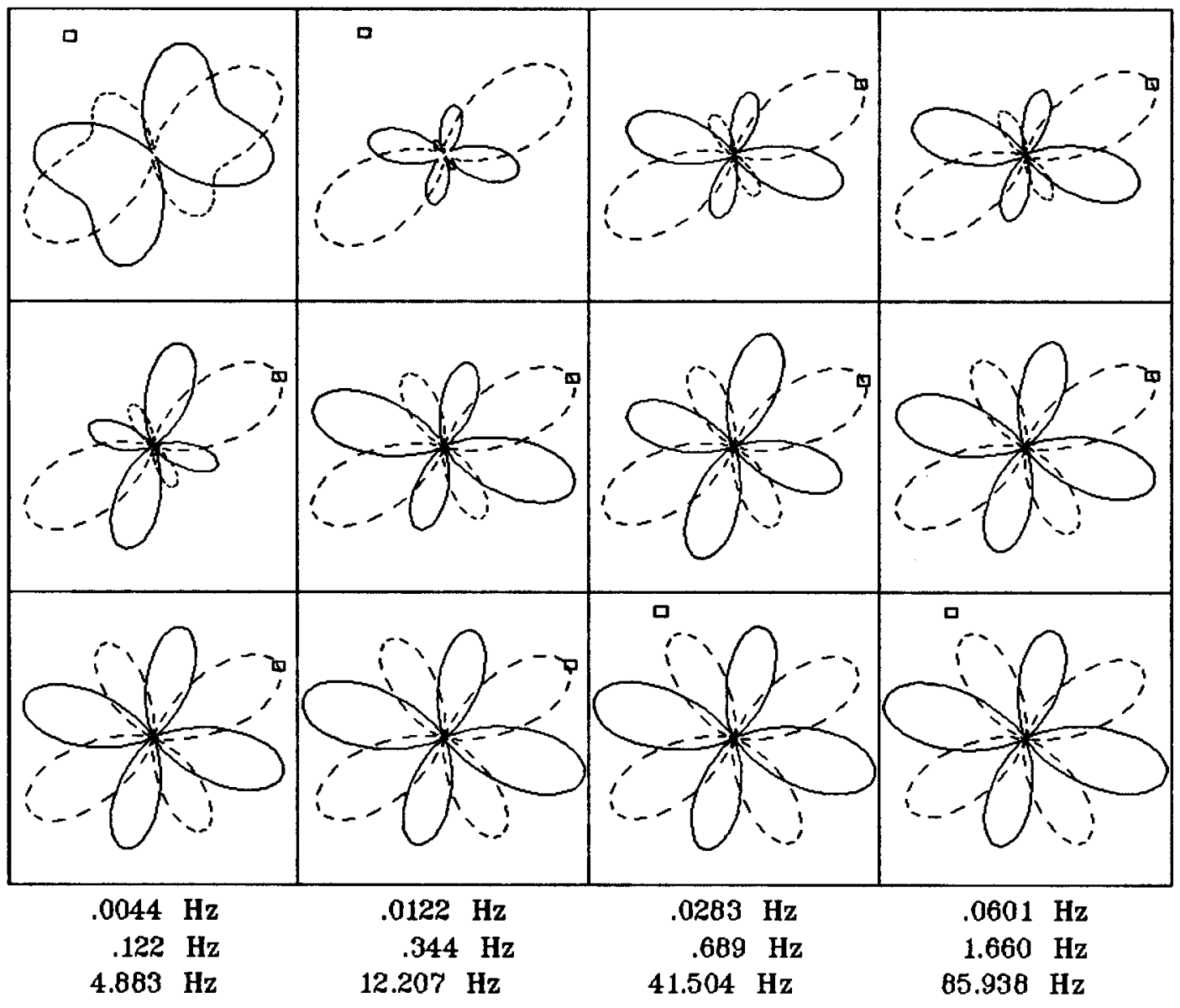

\section{Client:}

Remote: E local ref.

Acquired: 12:0 Jul 21, 1997 Survey Co:
Rotation:

Filename: nn27a.all

Channels: Ch6 Ch7 Ch8 Ch9 Ch10Ch1 Ch2 Plotted: 08:43 Dec 08, 2000

< EMI - ElectroMagnetic Instruments > 
TIPPER MAGNITUDE

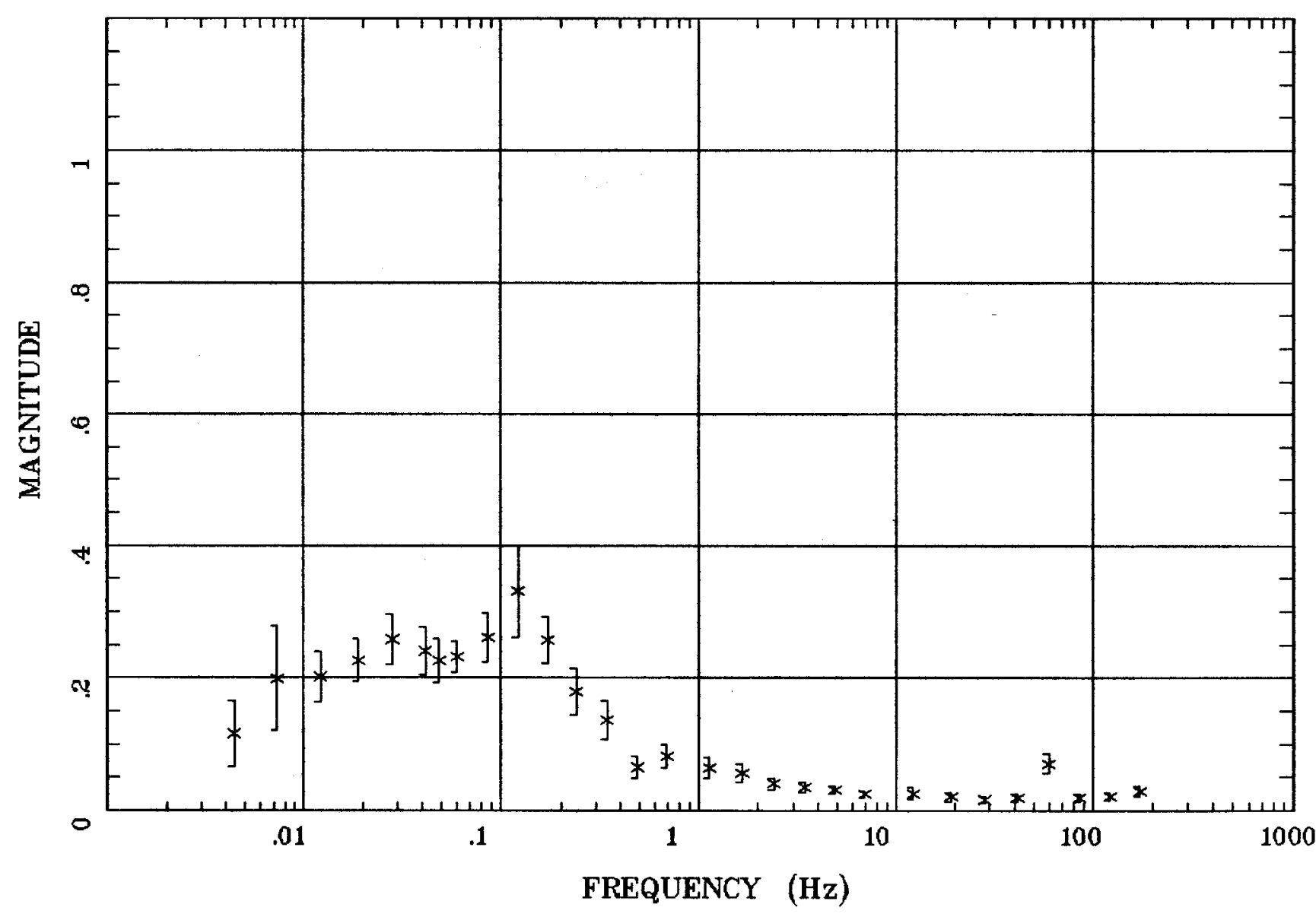

Client:

Remote: E local ref.

Acquired: 12:0 Jul 21, 1997

Survey Co:
...Battle Mtn...

Rotation:

Filename: nn27a.all

Channels: Ch6 Ch7 Ch8 Ch9 Ch10Ch1 Ch2 Plotted: 08:44 Dec 08, 2000

$<$ EMI - ElectroMagnetic Instruments 
...Battle Mtn...

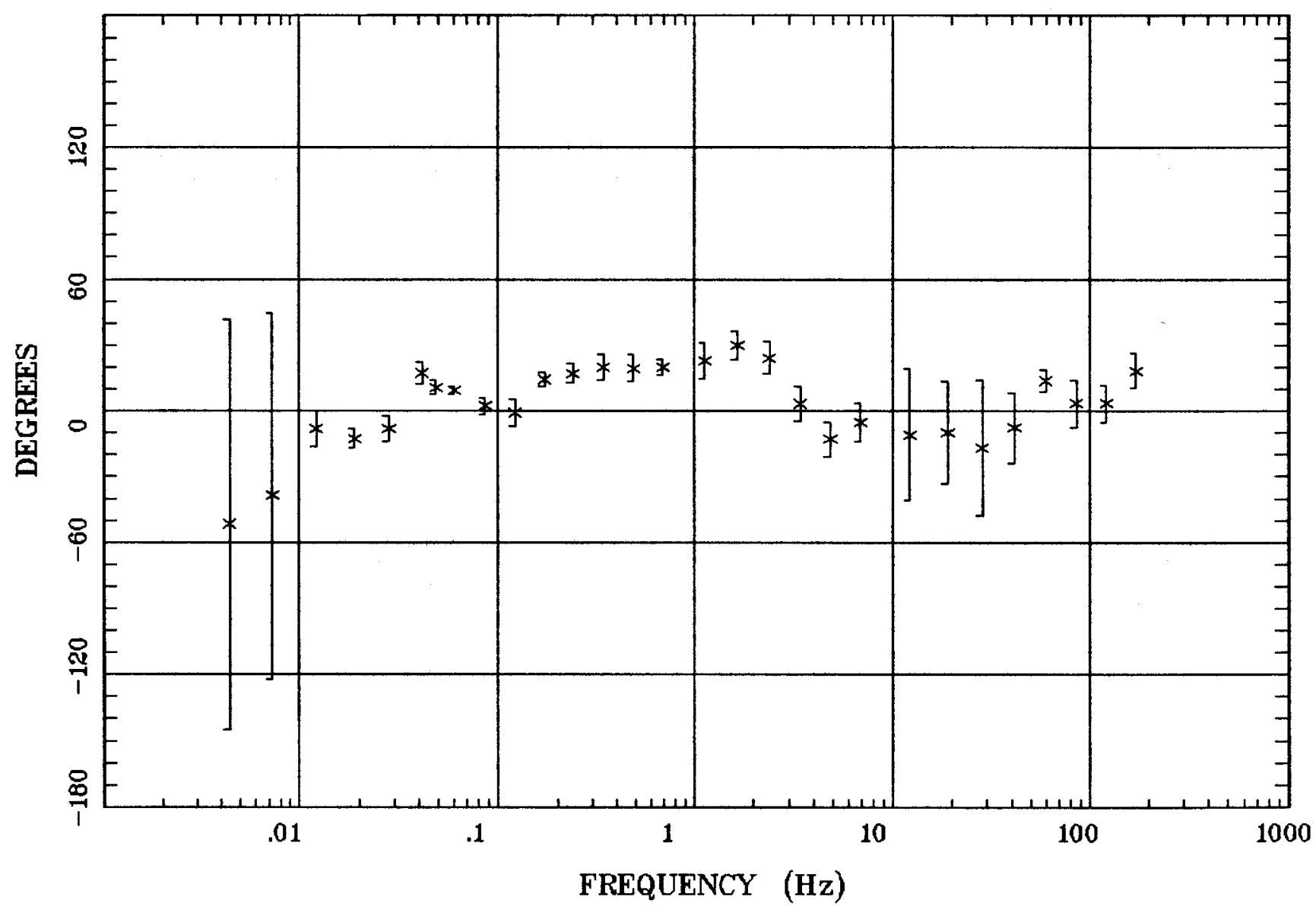

Client:

Remote: E local ref.

Acquired: 12:0 Jul 21, 1997 Survey Co:
Rotation:

Filename: nn27a.all

Channels: Ch6 Ch7 Ch8 Ch9 Ch10Ch1 Ch2

Plotted: 08:44 Dec 08, 2000

< EMI - ElectroMagnetic Instruments 


\section{Station 27A}

HzHx.x Coh HzHy.o

...Battle Mtn...

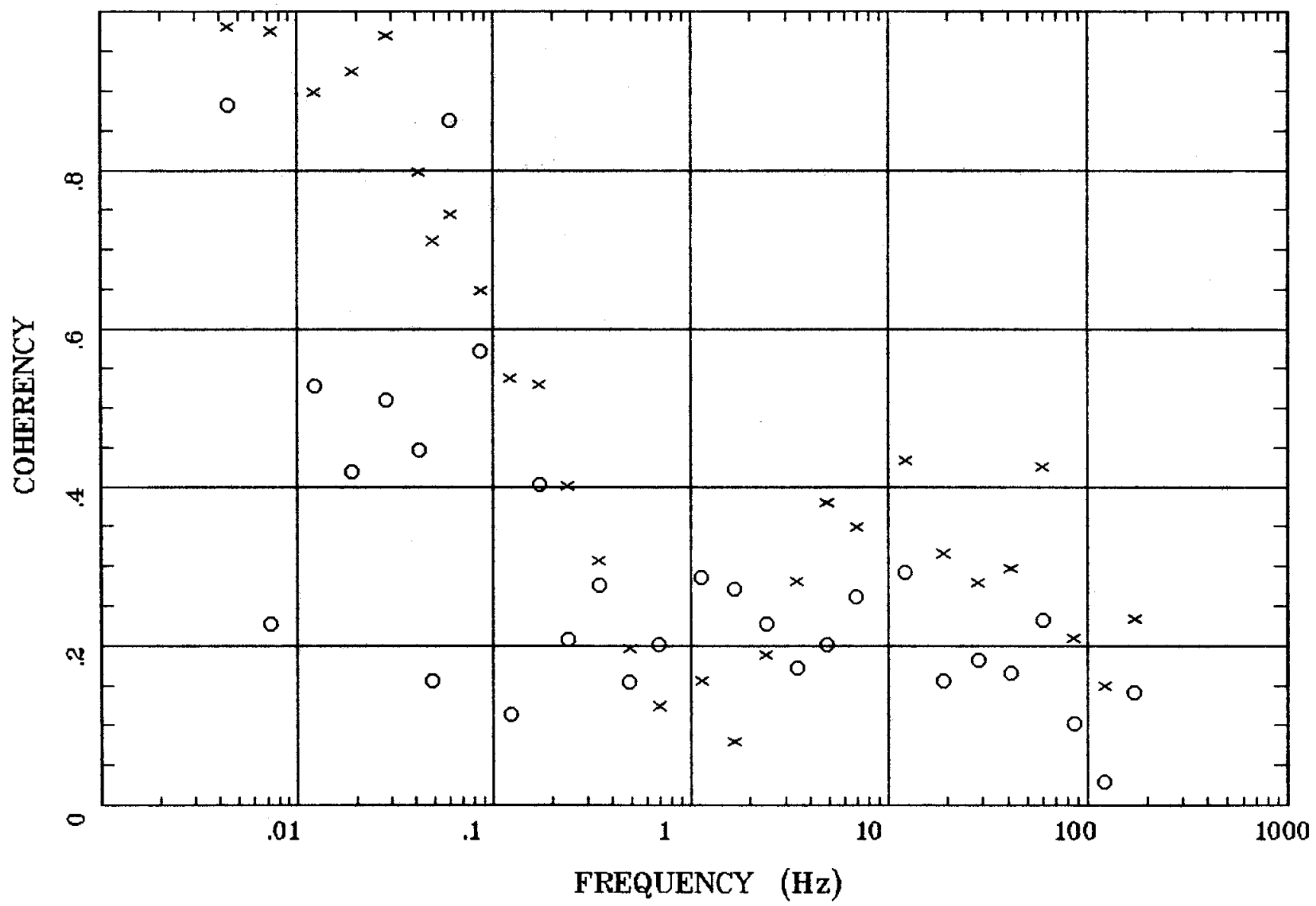

Client:

Remote: E local ref.

Acquired: 12:0 Jul 21, 1997

Survey Co:
Rotation:

Filename: nn27a.all

Channels: Ch6 Ch7 Ch8 Ch9 Ch10Ch1 Ch2

Plotted: 08:44 Dec 08, 2000

$<$ EMI - ElectroMagnetic Instruments > 


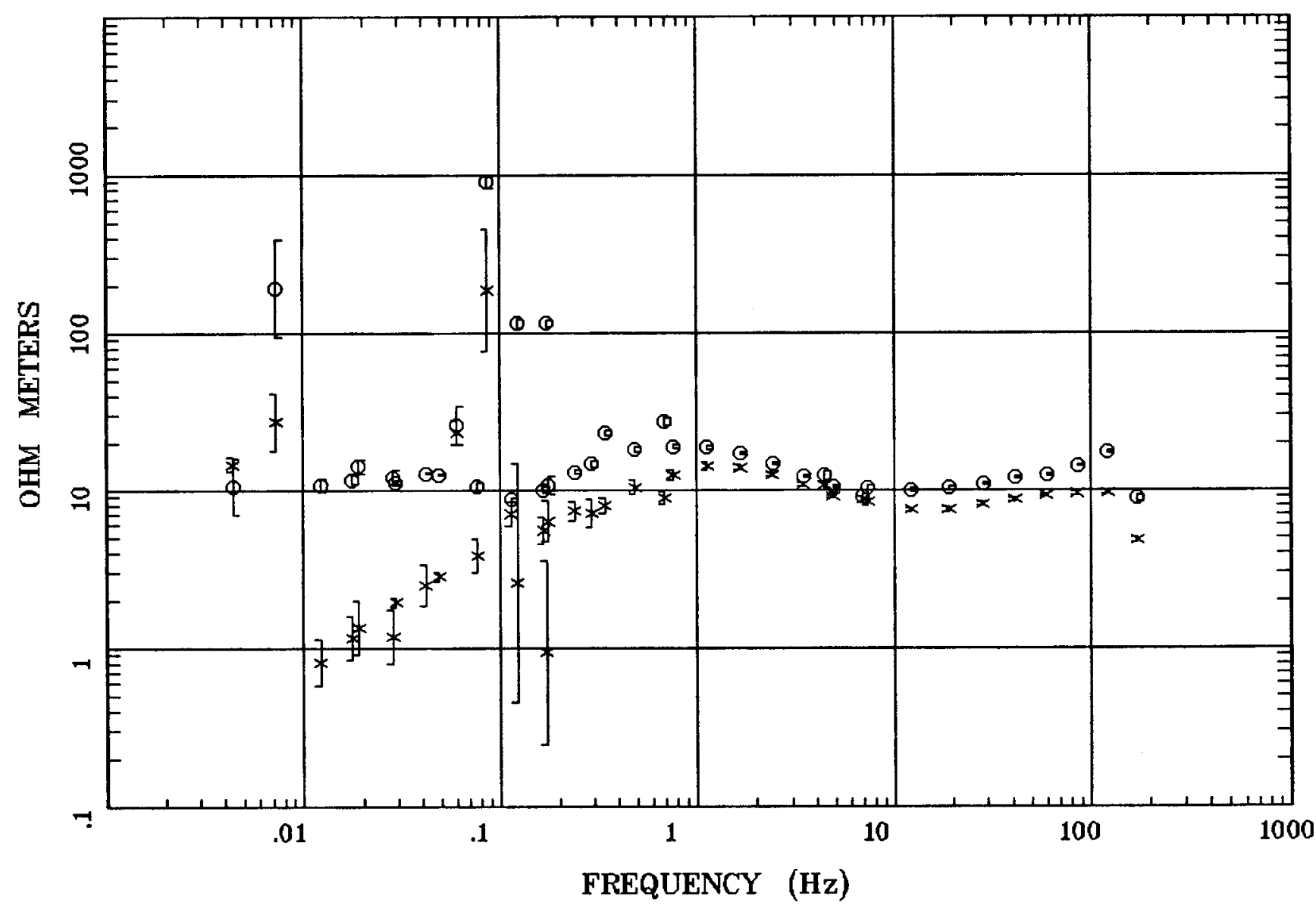

Client:

Remote: E local ref.

Acquired: 18:4 Jul 21, 1997 Survey Co:
Rotation:

Filename: nn28.all

Channels: Ch6 Ch7 Ch8 Ch9 Ch10Ch1 Ch2

Plotted: 08:44 Dec 08, 2000

$<$ EMI - ElectroMagnetic Instruments 


\section{IMPEDANCE PHASE}

...Battle Mtn...

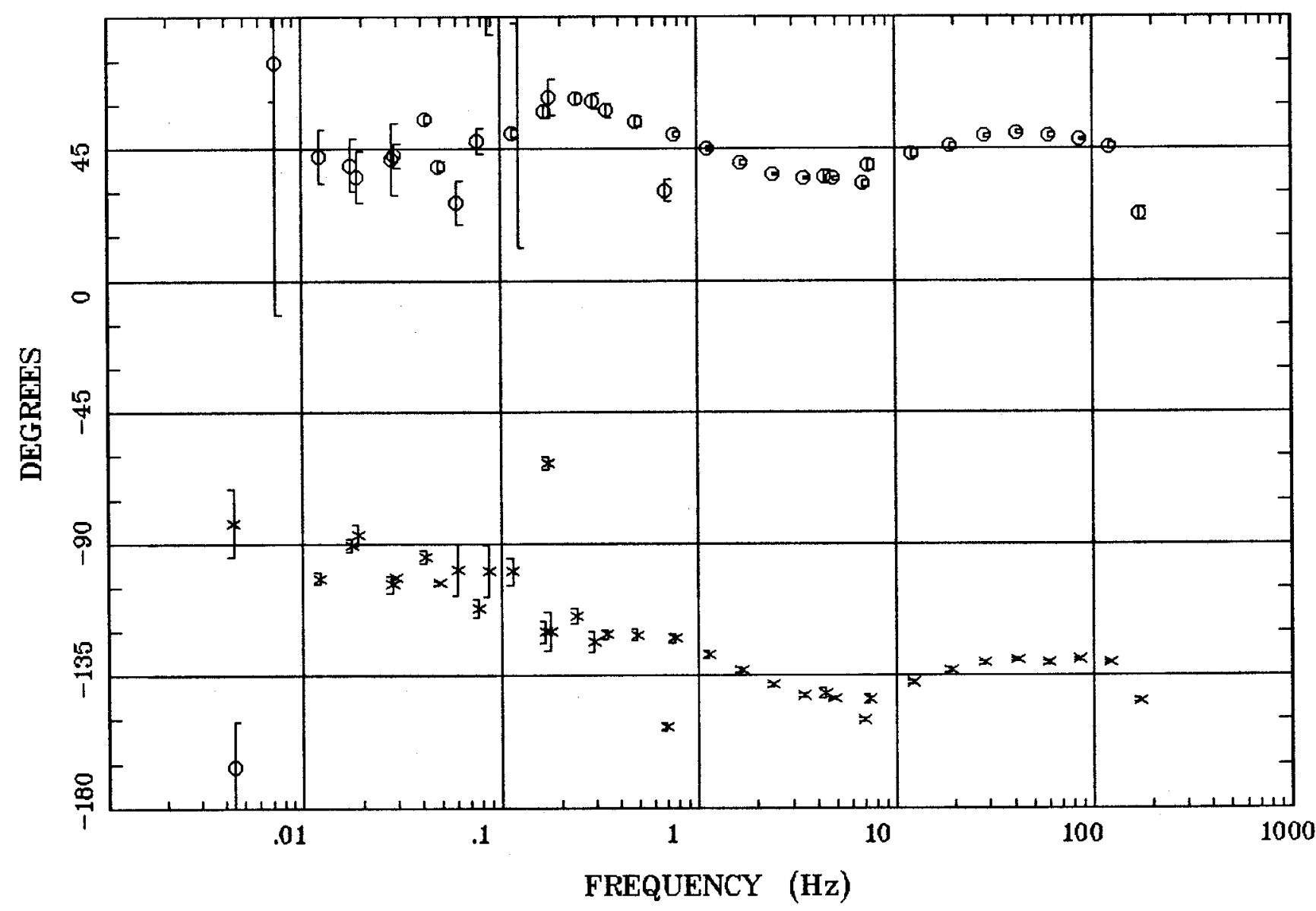

Client:

Remote: E local ref.

Acquired: 18:4 Jul 21, 1997

Survey Co:
Rotation:

Filename: nnz8.all

Channels: Ch6 Ch7 Ch8 Ch9 Ch10Ch1 Ch2

Plotted: 08:44 Dec 08, 2000

< EMI - ElectroMagnetic Instruments 
Station 28A

ROTATION ANGLE

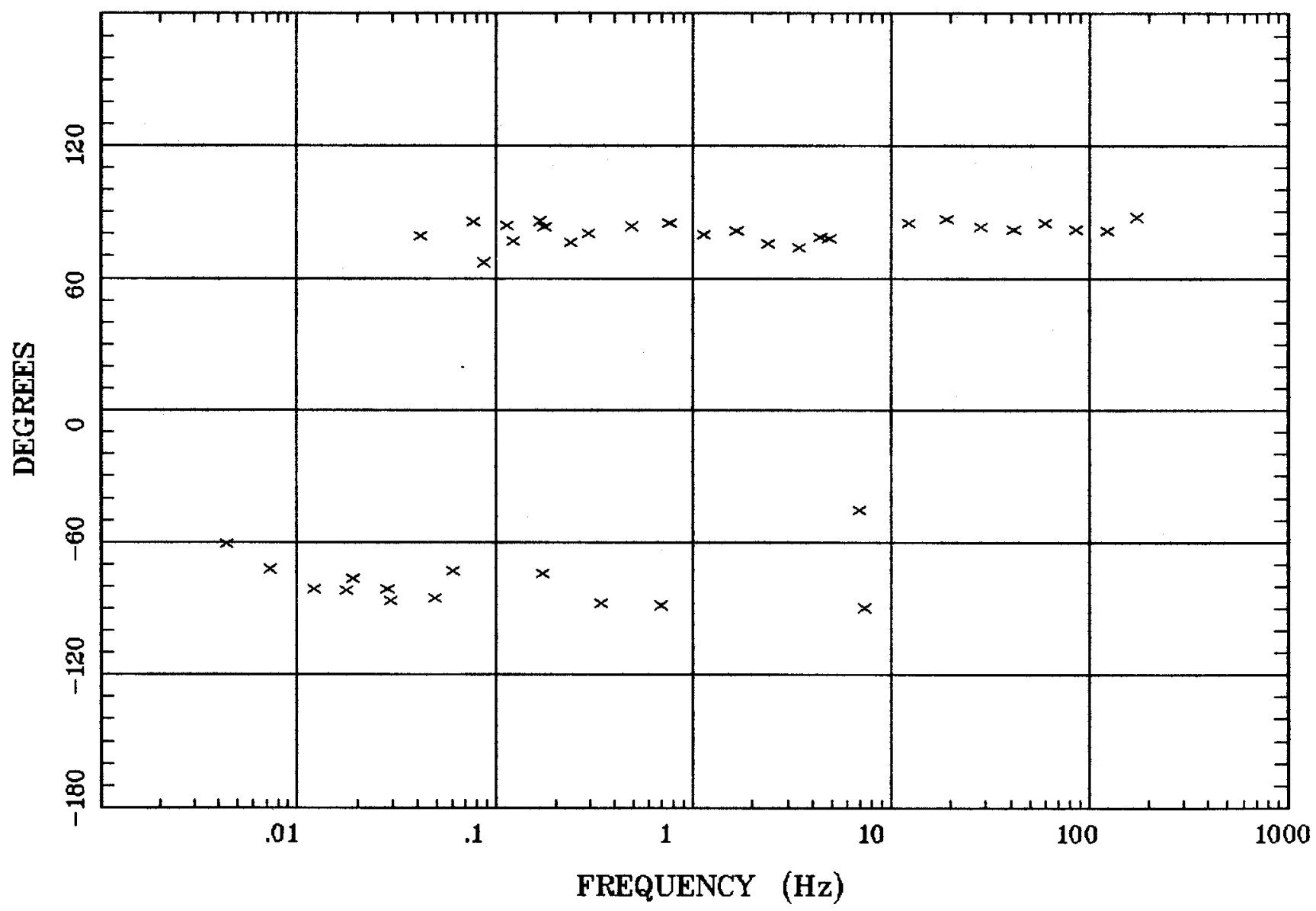

Client:

Remote: E local ref.

Acquired: 18:4 Jul 21, 1997

Survey Co:
Rotation:

Filename: nn28.all

Channels: Ch6 Chr ch8 Ch9 Ch10Ch1 Ch2

Plotted: 08:44 Dec 08, 2000

< EMI - ElectroMagnetic Instruments > 
IMPEDANCE SKEW

...Battle Mtn...

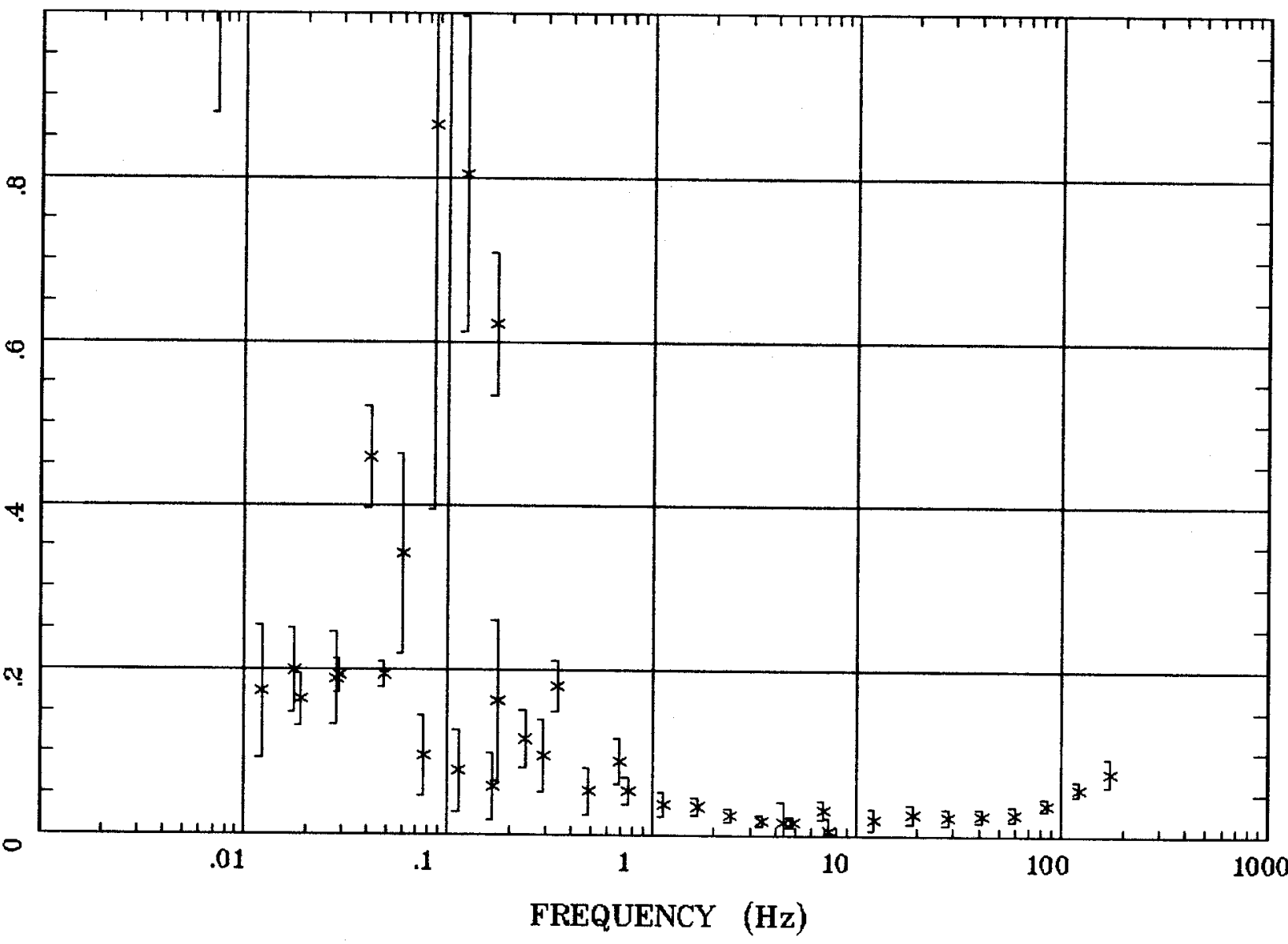

Client:

Remote: $E$ local ref.

Acquired: 18:4 Jul 21, 1997

Survey Co:
Rotation:

Filename: nnz8.all

Channels: Ch6 Ch7 Ch8 Ch9 Ch10Ch1 Ch2

Plotted: 08:44 Dec 08, 2000

$<$ EMI - ElectroMagnetic Instruments > 
Station 28A

\section{E MULT Coh.}

...Battle Mtn...

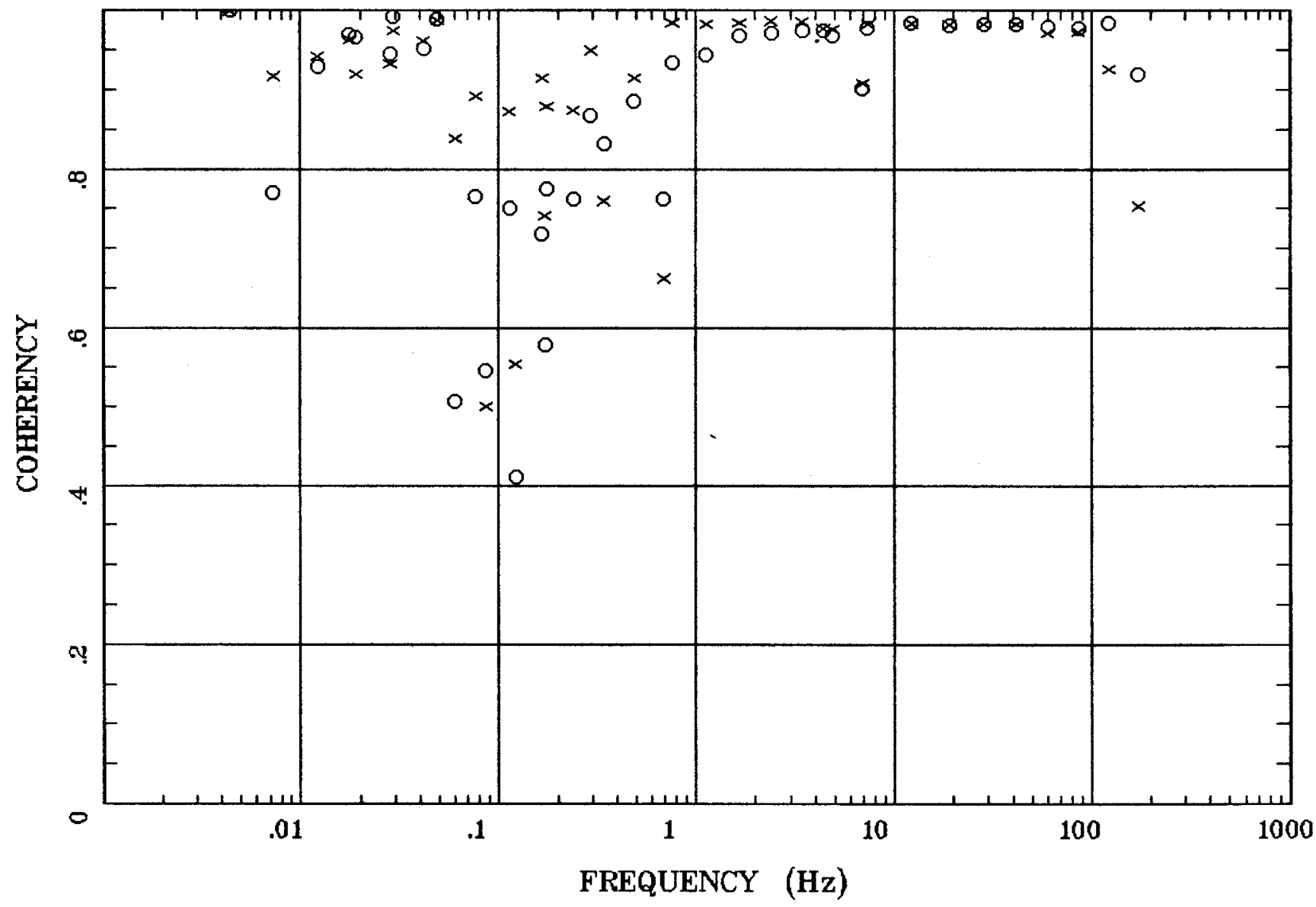

Client:

Remote: E local ref.

Acquired: 18:4 Jul 21, 1997

Survey Co:

Rotation:

Filename: nn28.ell

Channels: Ch6 Ch7 Ch8 Ch9 Ch10Ch1 Ch2

Plotted: 08:44 Dec 08, 2000

< EMI - ElectroMagnetic Instruments 


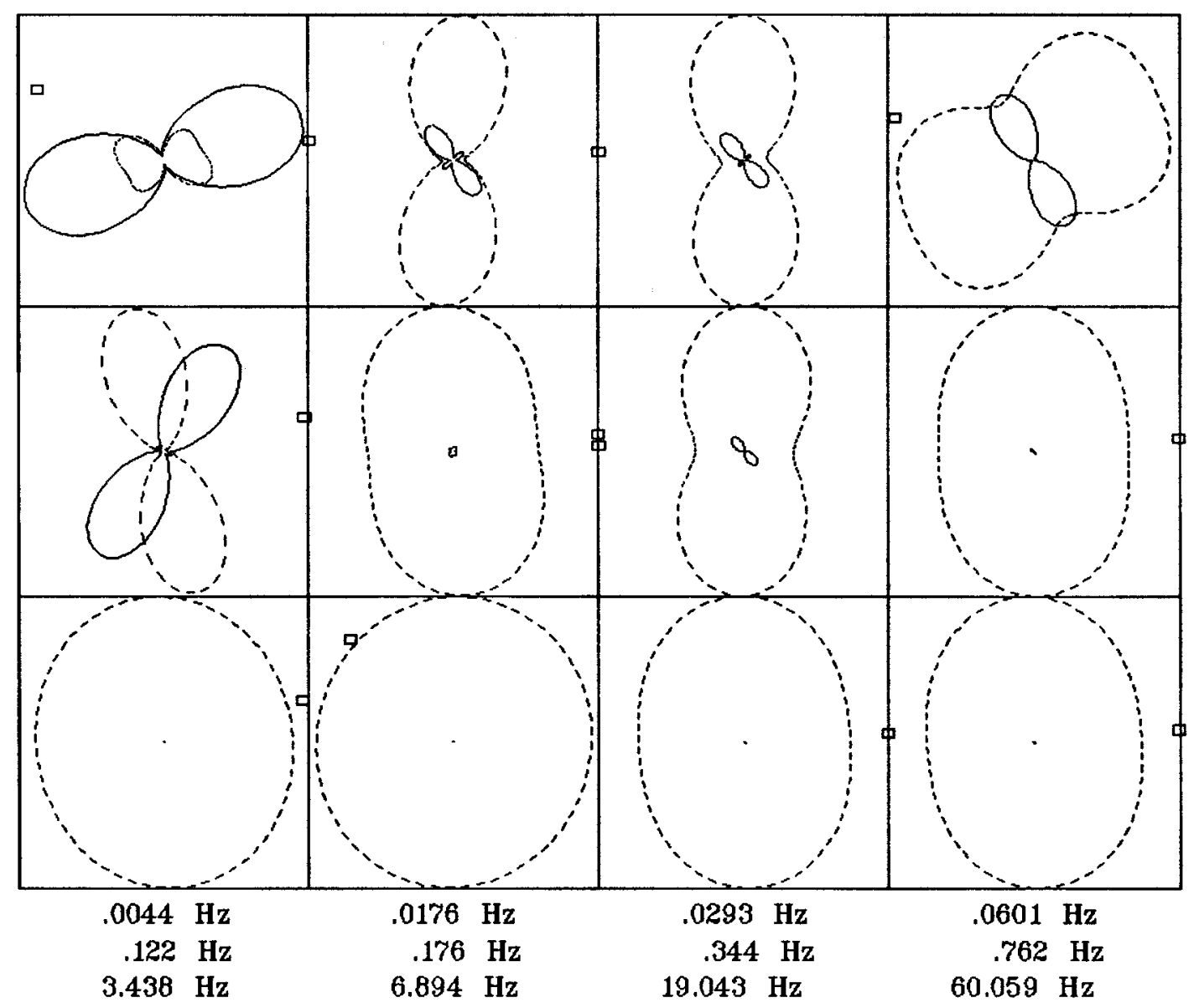

Client:

Remote: E local ref.

Acquired: 18:4 Jul 21, 1997 Survey Co:
Rotation:

Filename: nnz8.all

Channels: Ch6 Ch7 Ch8 Ch9 Ch10Ch1 Ch2

Plotted: 08:45 Dec 08, 2000

< EMI - ElectroMagnetic Instruments 
Station 28A

TIPPER MAGNITUDE

...Battle Mtn...

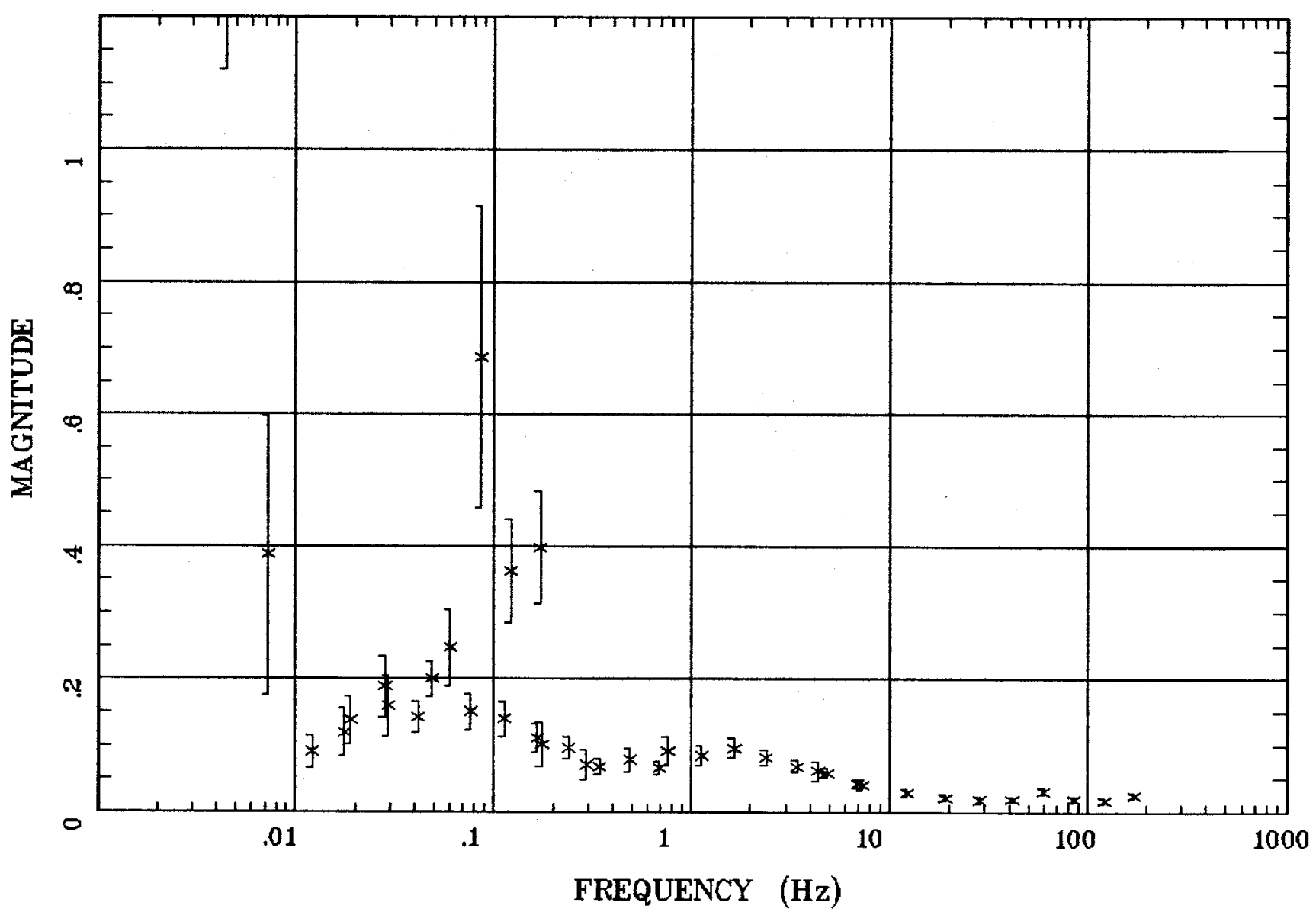

Client:

Rotation:

Remote: E local ref.

Acquired: 18:4 Jul 21, 1997

Filename: nn28.all

Channels: Ch6 Ch7 Ch8 Ch9 Ch10Ch1 Ch2

Plotted: 08:45 Dec 08, 2000

Survey Co:

< EMI - ElectroMagnetic Instruments > 
TIPPER STRIKE

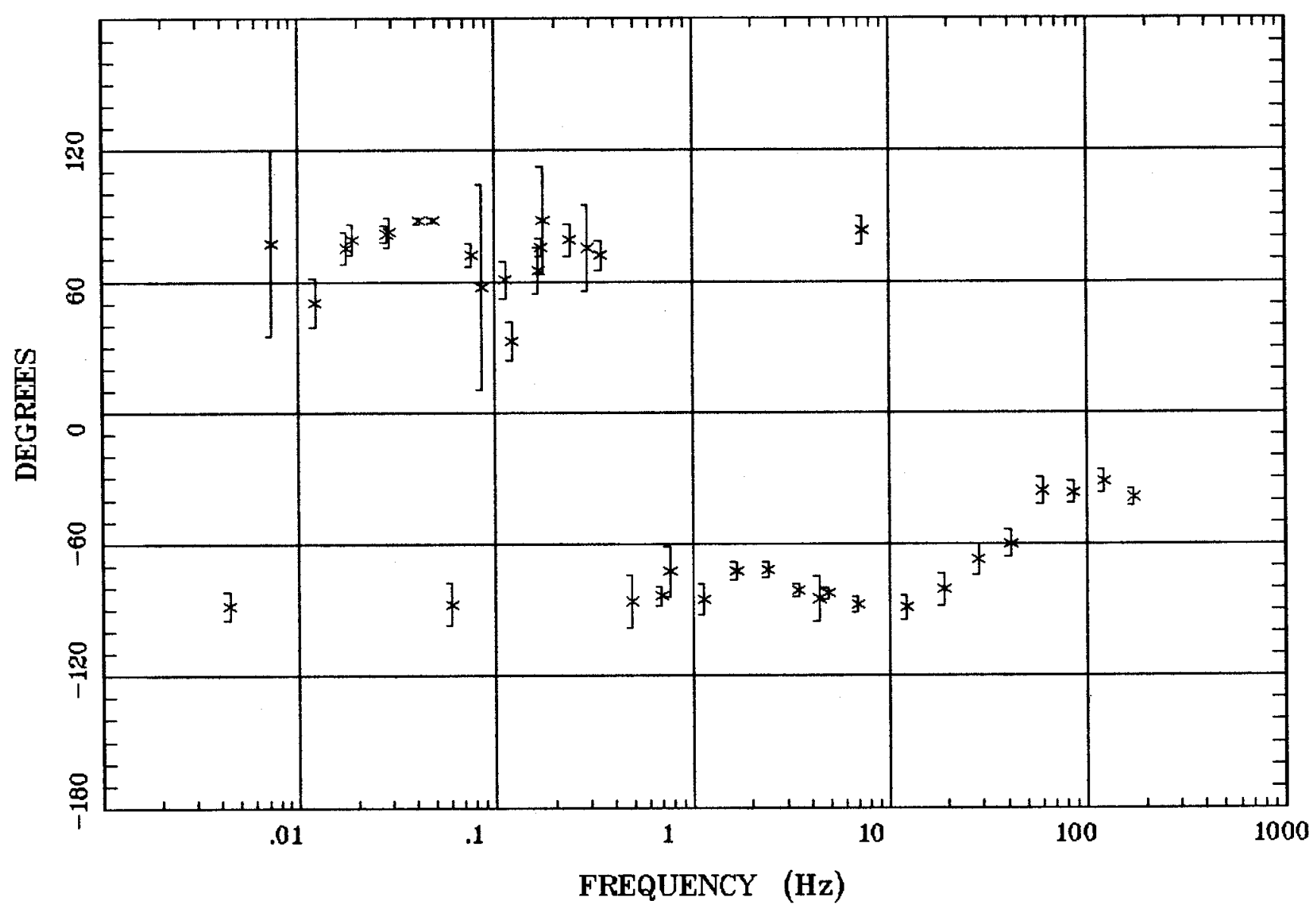

Client:

Remote: E local ref.

Acquired: 18:4 Jul 21, 1997 Survey Co:

\section{Rotation:}

Filename: nnze.all

Channels: Ch6 Ch7 Ch8 Ch9 Ch10Ch1 Ch2 Plotted: 08:45 Dec 08, 2000

< EMI - ElectroMagnetic Instruments 
HzHx.x Coh HzHy.o

...Battle Mtn...

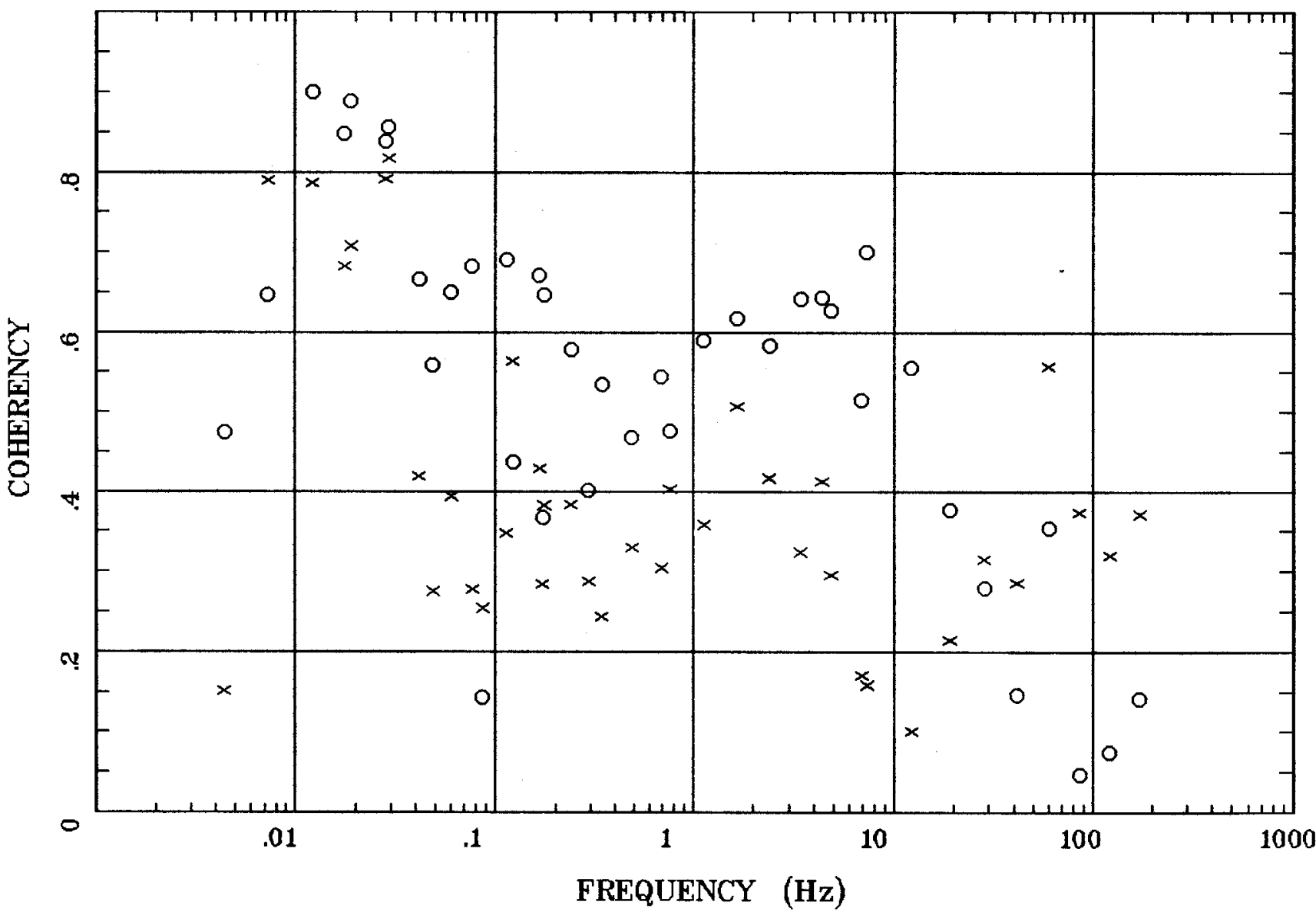

Client:

Remote: E local ref.

Acquired: 18:4 Jul 21, 1997 Survey Co:
Rotation:

Filename: nn28.all

Channels: Ch6 Ch7 Ch8 Ch9 Ch10Ch1 Ch2 Plotted: 08:45 Dec 08, 2000

< EMI - ElectroMagnetic Instruments > 


\section{Station 1}

APPARENT RESISTIVITY

Carlin, Nevada

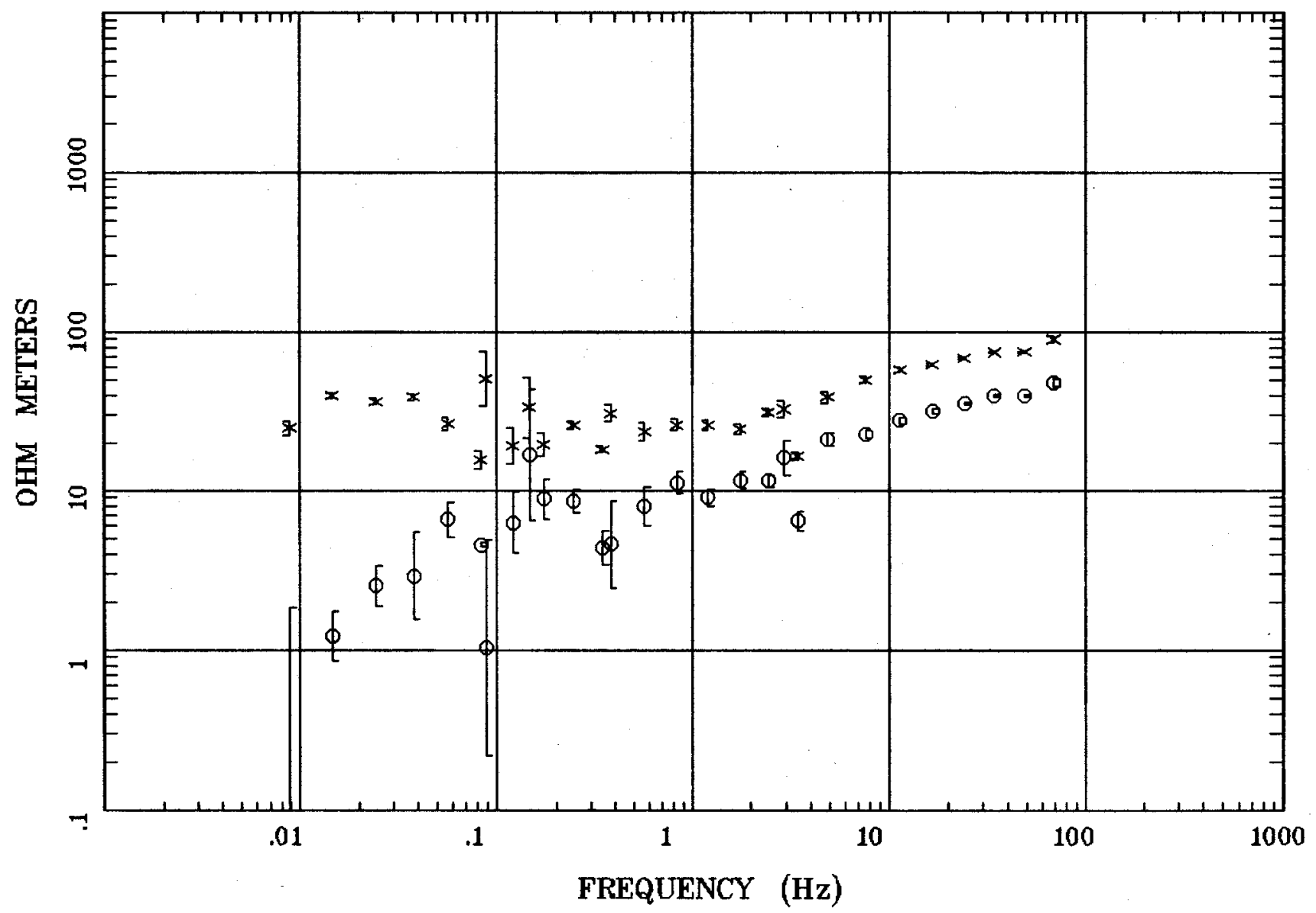

Client:

Remote: local

Acquired: 13:4 Jul 26, 1999

Survey Co:USGS
Rotation:

Filename: ct01.avg

Channels: Ch1 Ch2 Ch3 Ch4 Ch5 Ch8 Ch9

Plotted: 15:42 Jan 24, 2001

< EMI - ElectroMagnetic Instruments > 


\section{Station 1}

IMPEDANCE PHASE

Carlin, Nevada

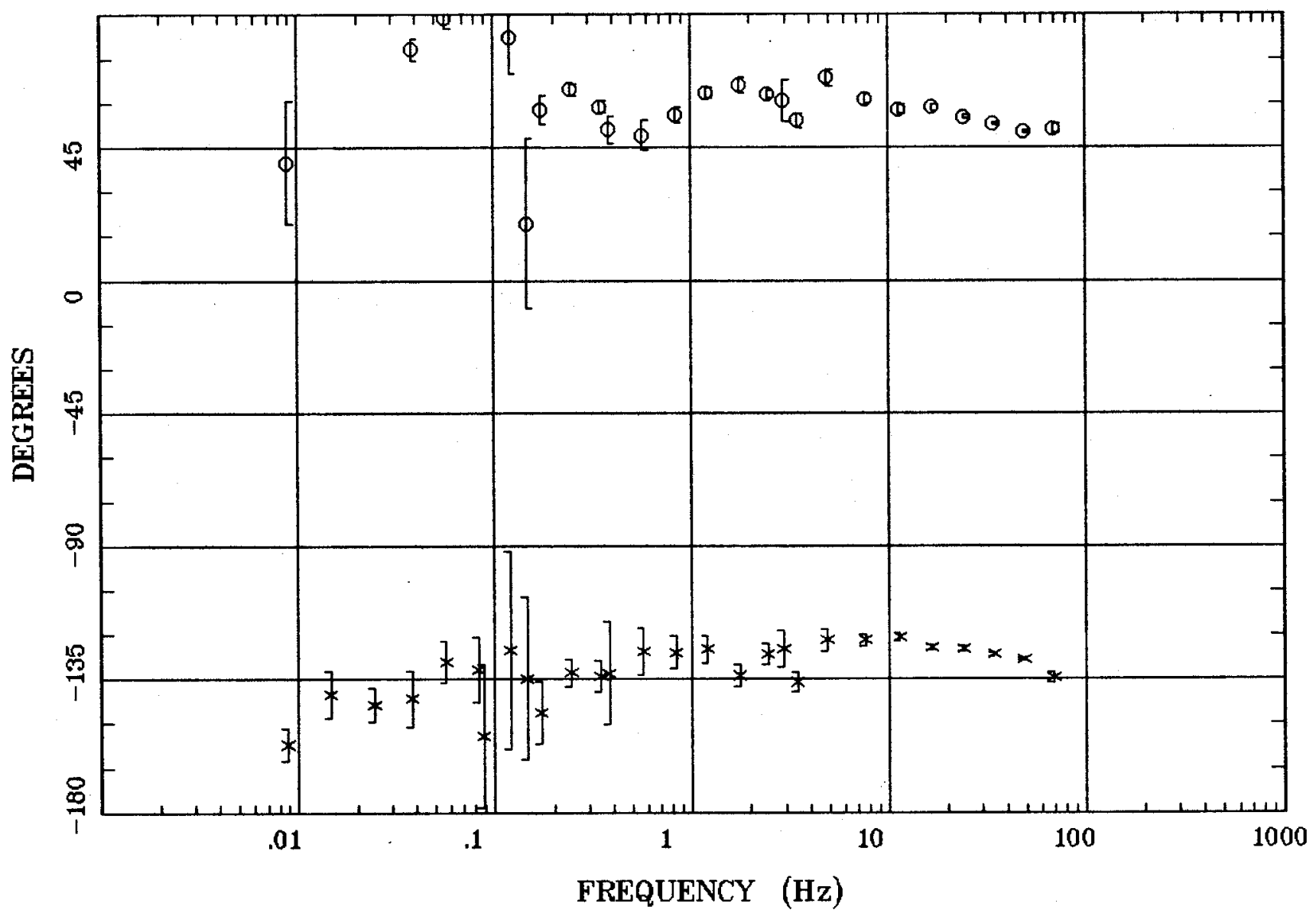

Client:

Remote: locel

Acquired: 13:4 Jul 26, 1999

Survey Co:USGS
Rotation:

Filename: ct01.avg

Channels: Ch1 Ch2 Ch3 Ch4 Ch5 Ch8 Ch9

Plotted: 15:42 Jan 24, 2001

< EMI - ElectroMagnetic Instruments > 


\section{Station 1}

ROTATION ANGLE

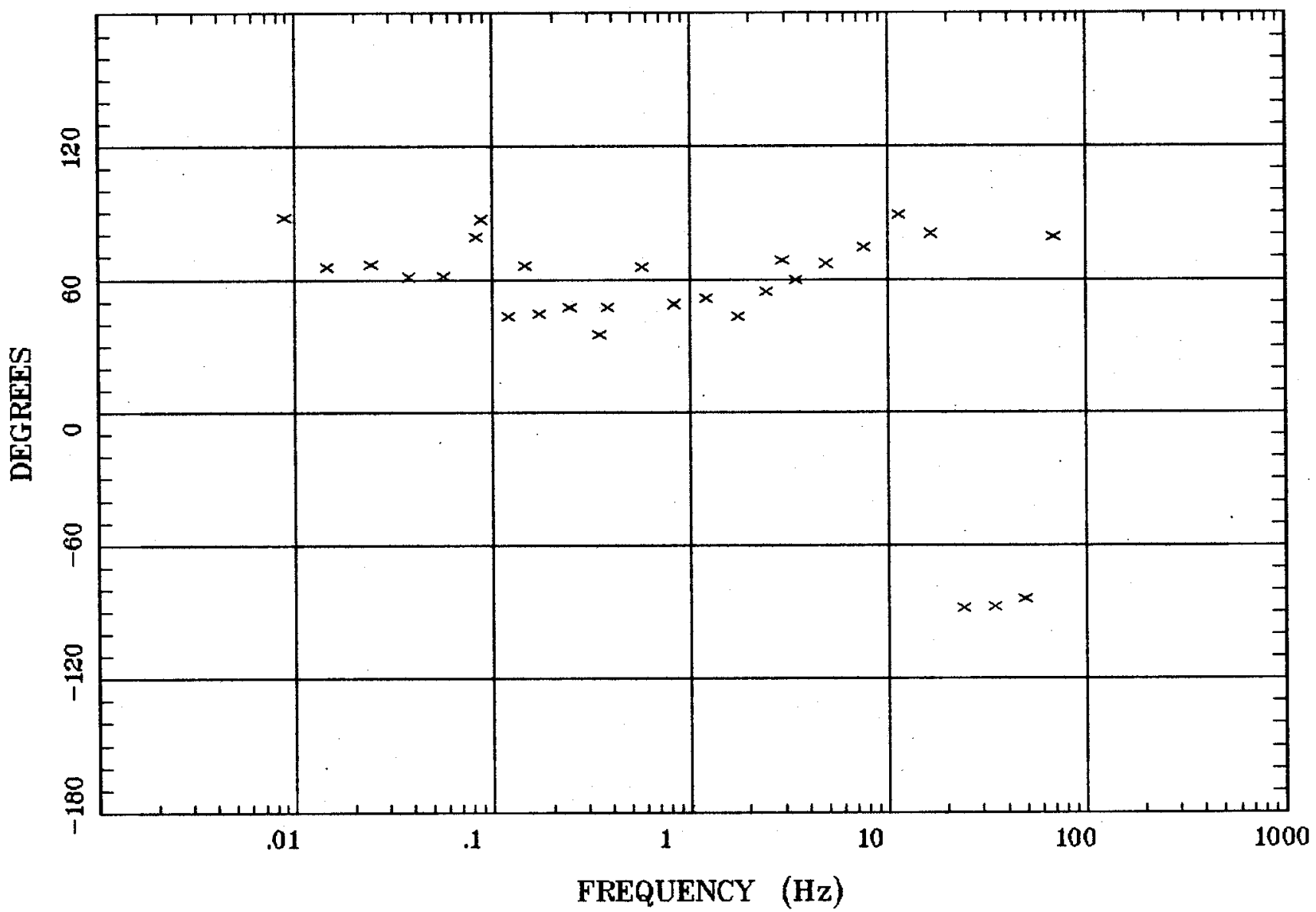

Client:

Remote: local

Acquired: 13:4 Jul 26, 1999

Survey Co:USGS
Rotation:

Filename: ct01.avg

Channels: Ch1 Ch2 Ch3 Ch4 Ch5 Ch8 Ch9

Plotted: 15:42 Jan 24, 2001

< EMI - ElectroMagnetic Instruments 
Carlin, Nevada

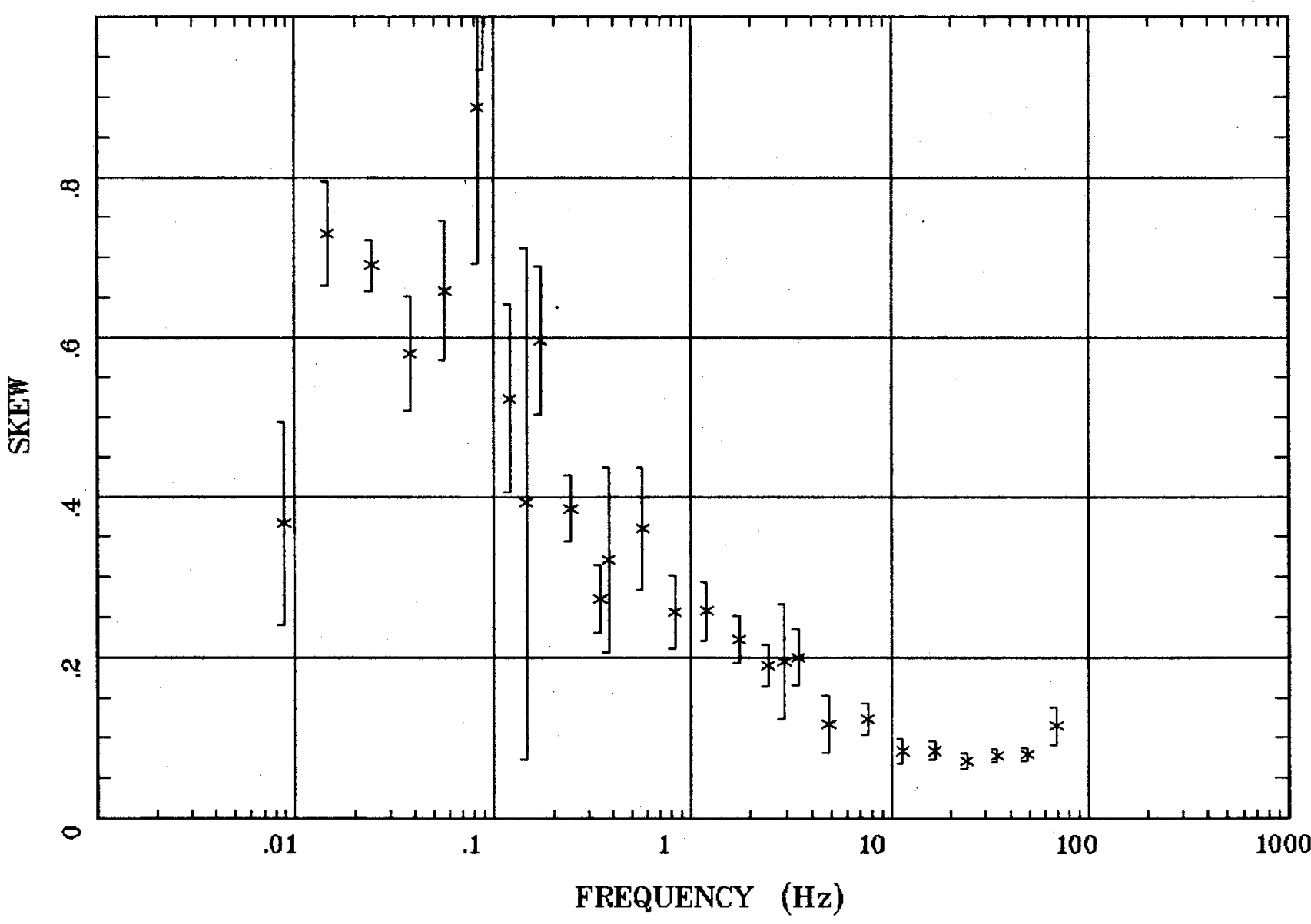

Client:

Remote: local

Acquired: 13:4 Jul 26, 1999

Survey Ca:USGS
Rotation:

Filename: ct01.avg

Channels: Ch1 Ch2 Ch3 ch4 Ch5 Ch8 Ch9

Plotted: 15:42 Jan 24, 2001

< EMI - ElectroMagnetic Instruments > 


\section{Station 1}

E MULT Coh.

Carlin, Nevada

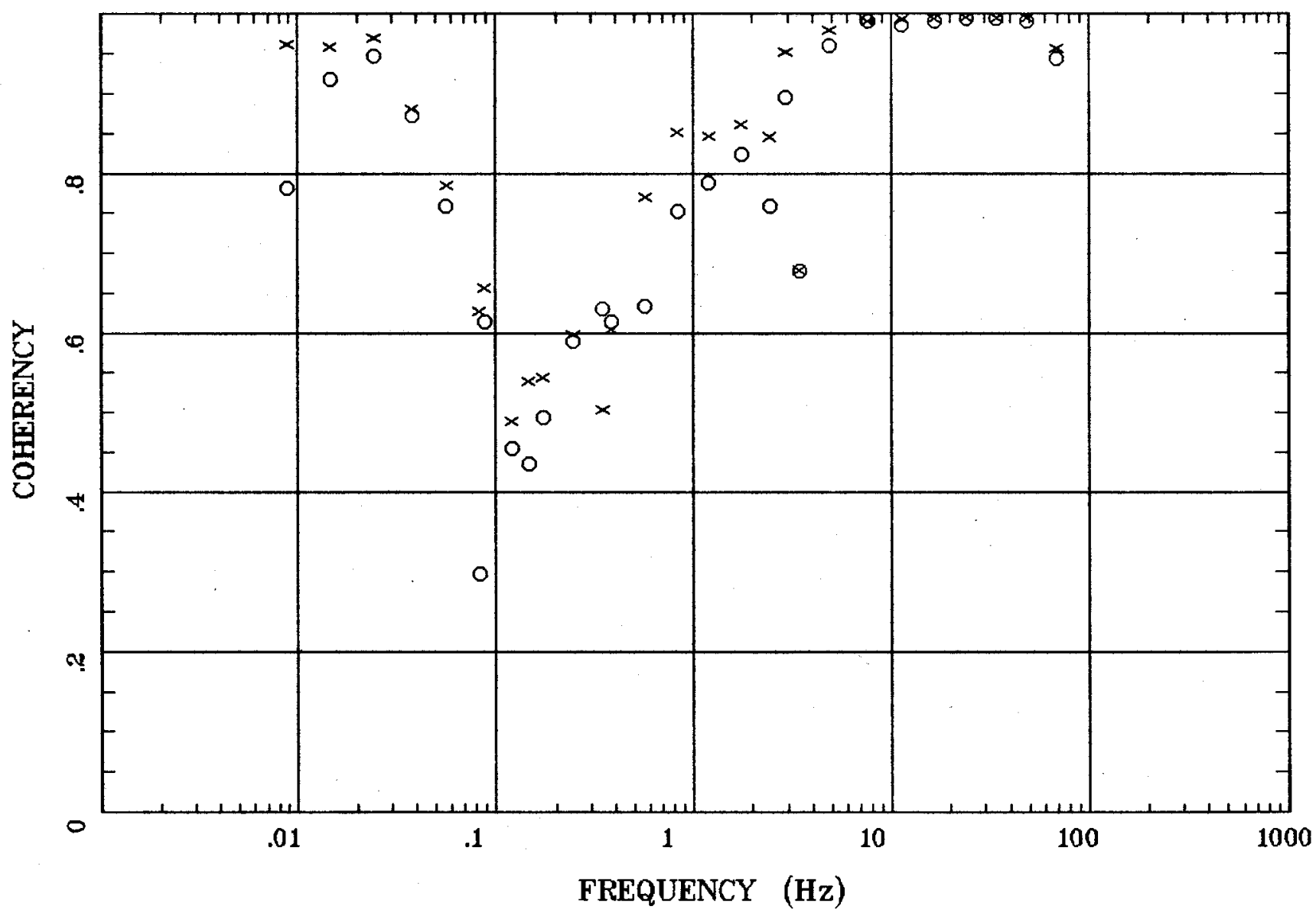

Client:

Remote: local

Acquired: 13:4 Jul 26, 1999

Survey Co:USGS
Rotation:

Filename: ct01.avg

Channels: Ch1 Ch2 Ch3 Ch4 Ch5 Ch8 Ch9

Plotted: 15:42 Jan 24, 2001

$<$ EMI - ElectroMagnetic Instruments > 


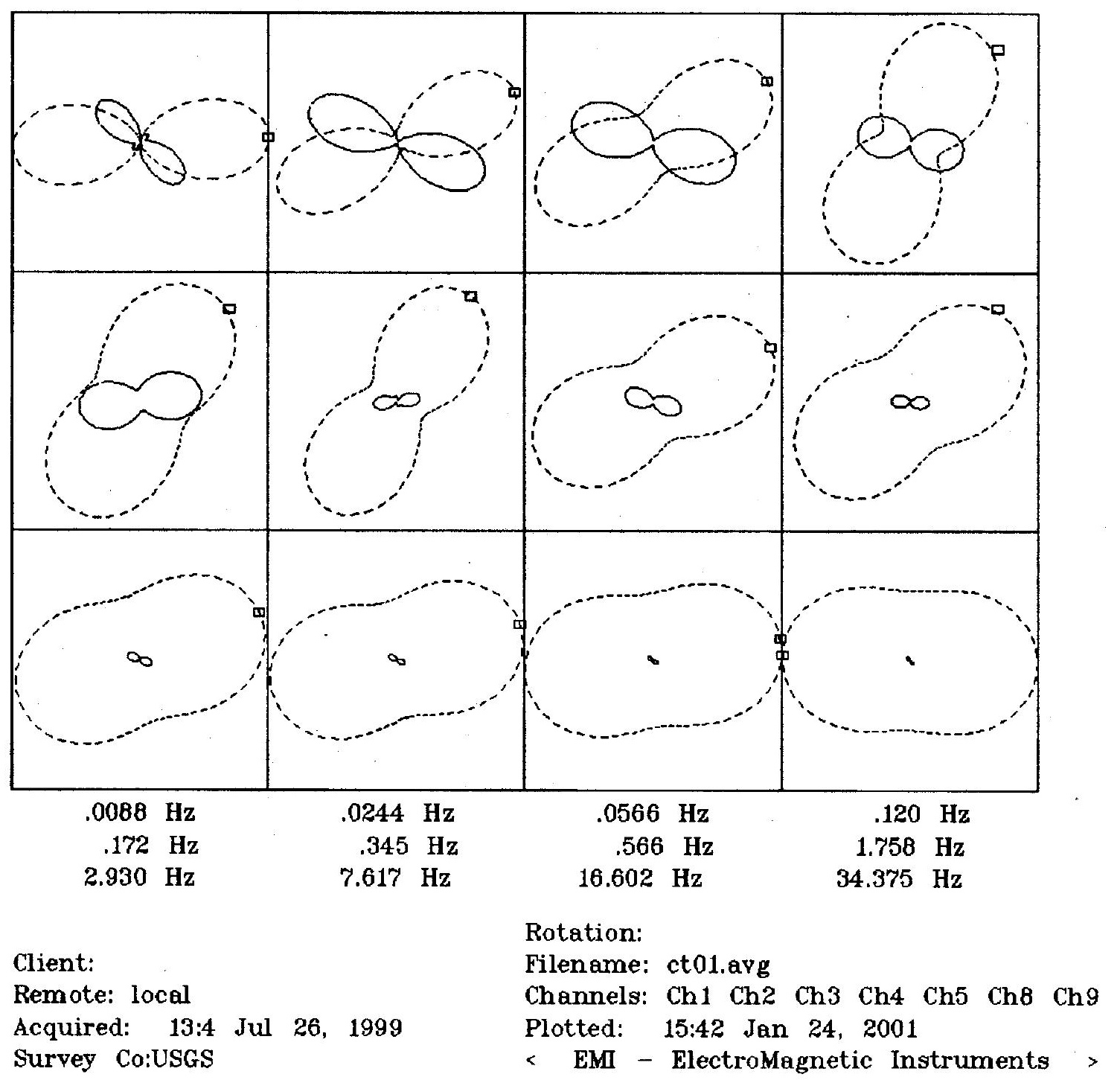


TIPPER MAGNITUDE

Carlin, Nevada

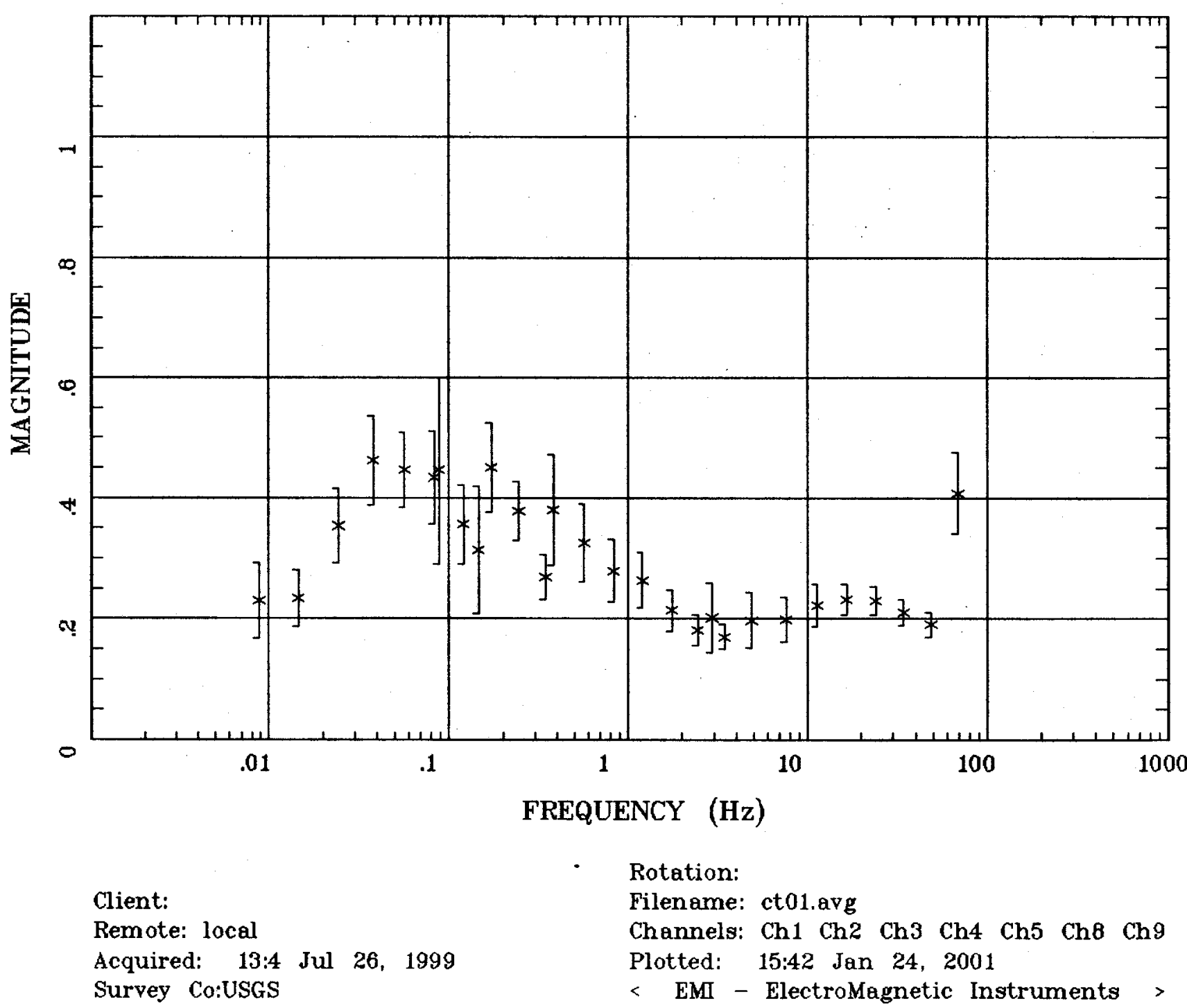




\section{Station 1}

TIPPER STRIKE

Carlin, Nevada

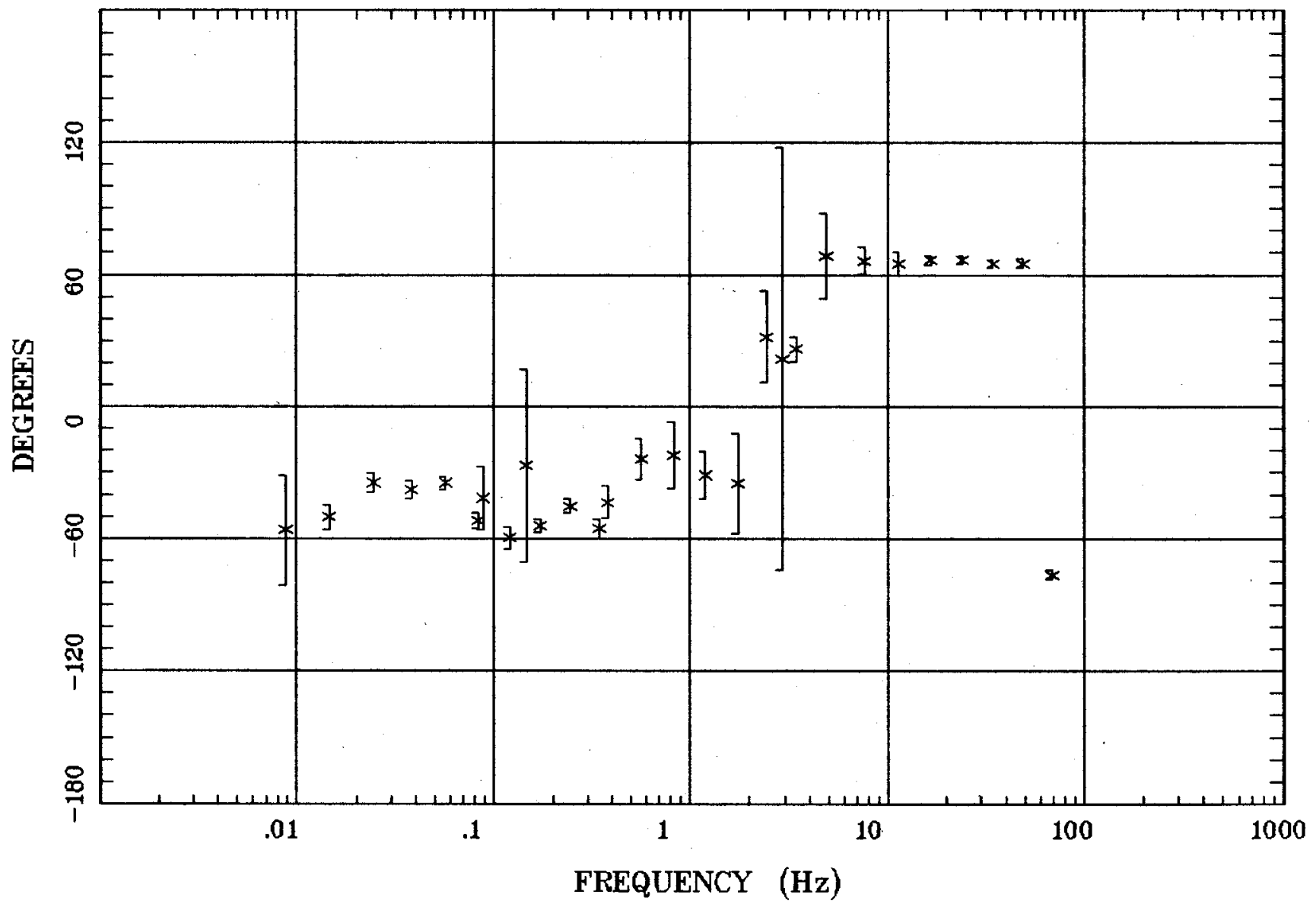

Client:

Rotation:

Filename: ct01.avg

Remote: local

Acquired: 13:4 Jul 26, 1999

Filename: cto1.avg

Channels: Ch1 Ch2 Ch3 ch4 Ch5 Ch8 Ch 9

Plotted: 15:42 Jan 24, 2001

Survey Co:USGS

< EMI - ElectroMagnetic Instruments 
HzHx.x Coh HzHy.o

Carlin, Nevada

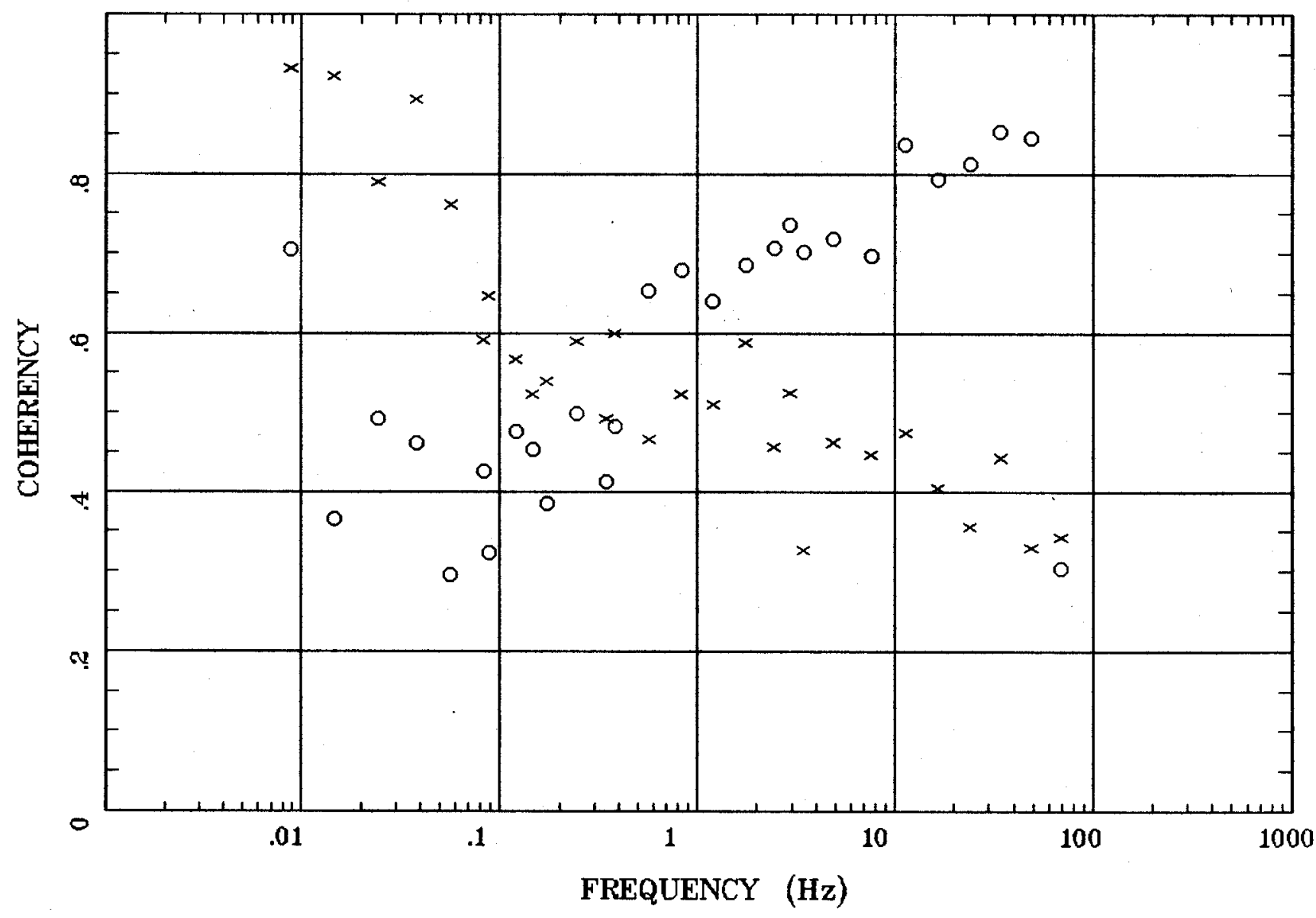

Client:

Remote: local

Acquired: 13:4 Jul 26, 1999

Survey Co:USGS
Rotation:

Filename: ct01.avg

Channels: Ch1 Ch2 Ch3 Ch4 Ch5 Ch8 Ch9

Plotted: 15:42 Jan 24, 2001

< EMI - ElectroMagnetic Instruments 


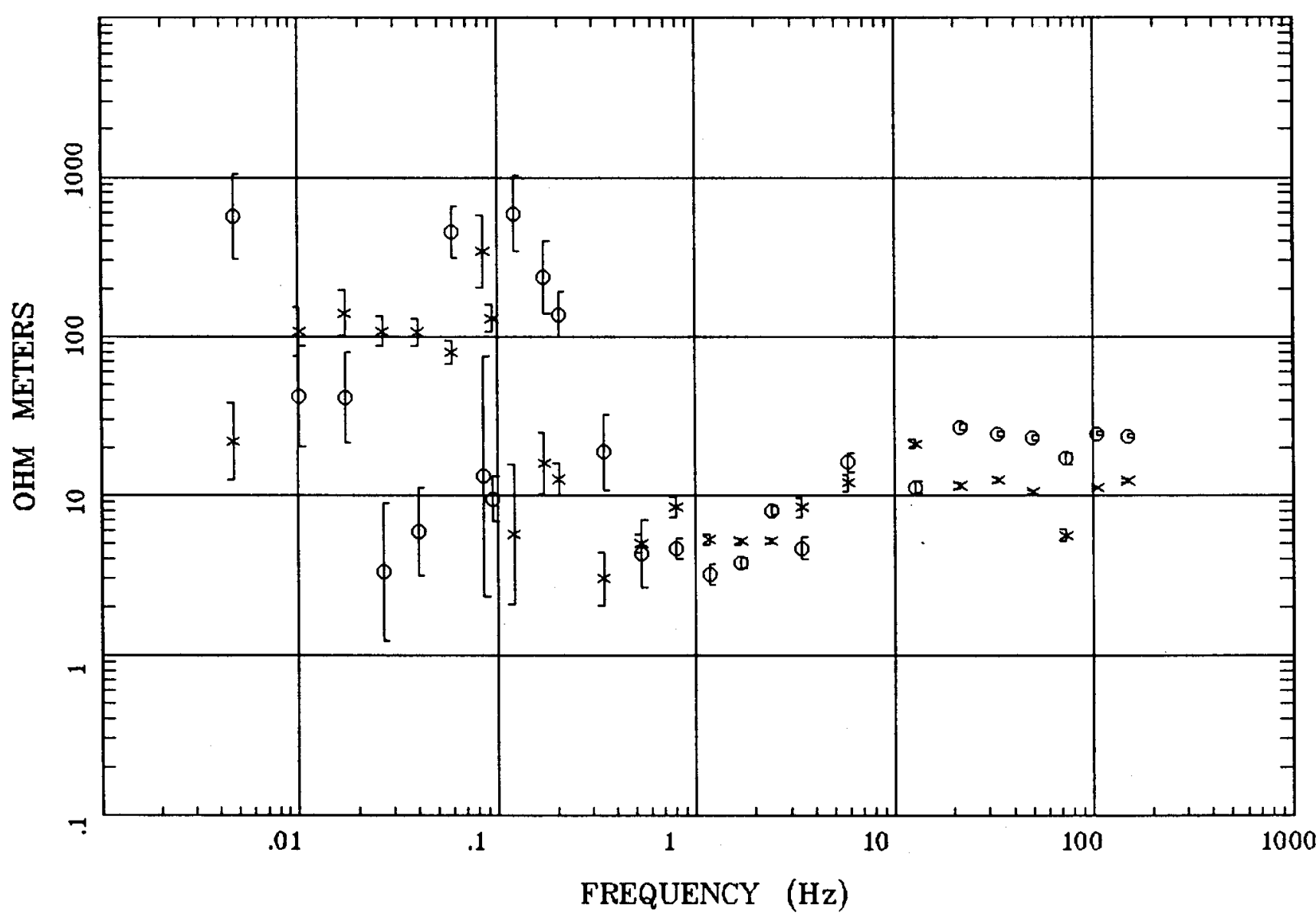

Client:

Remote:

Acquired:

Survey Co:
Rotation:

Filename: CT24C

Channels: Ch1 Ch2 Ch3 Ch4 Ch5 Ch6 Ch7

Plotted: 15:57 Dec 11, 2000

< EMI - ElectroMagnetic Instruments 
Station 24B

\section{IMPEDANCE PHASE}

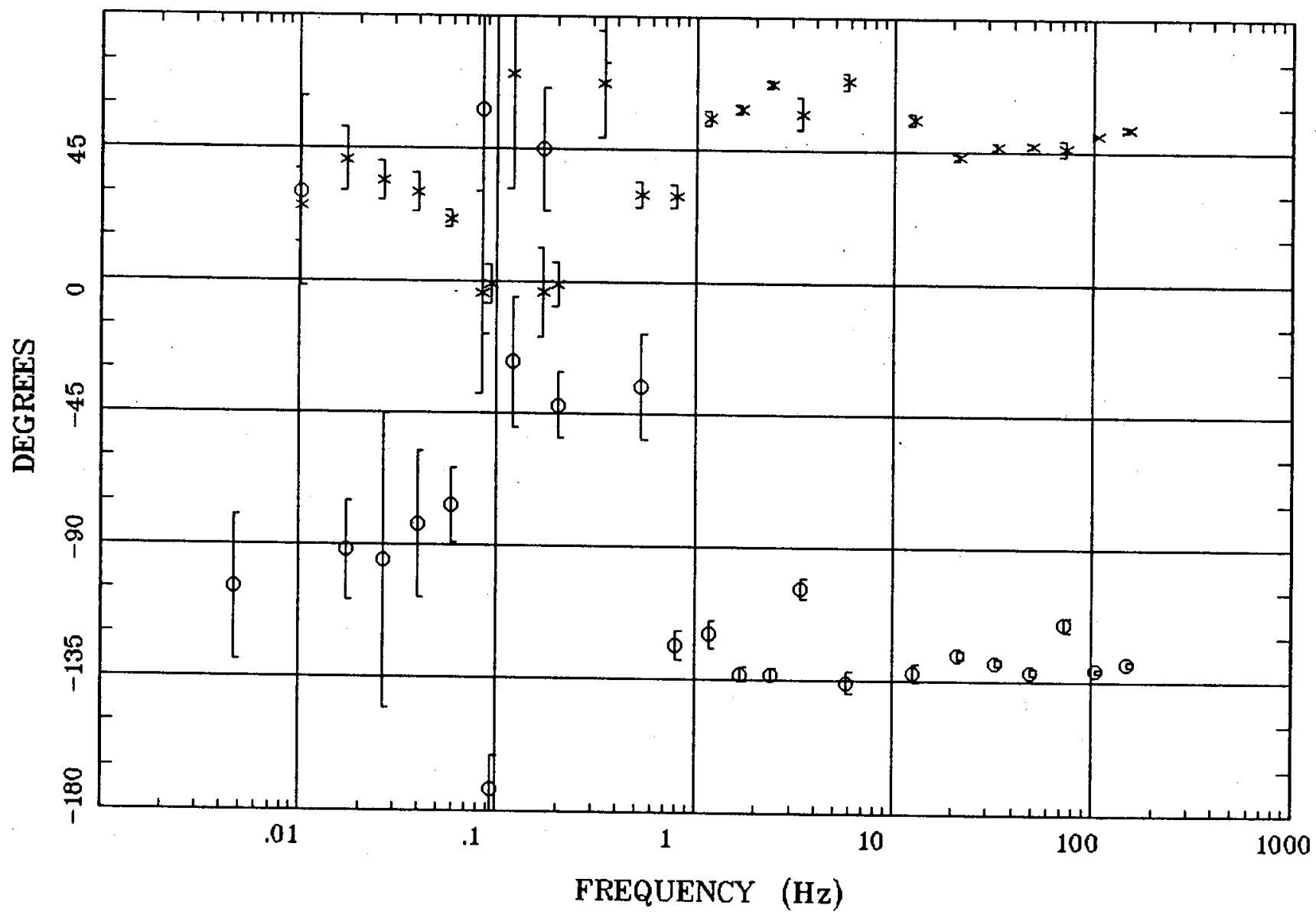

Client:

Remote:

Acquired:

Survey Co:
Rotation:

Filename: CT24C

Channels: Ch1 Ch2 Ch3 Ch4 Ch5 Ch6 Ch7

Plotted: 15:57 Dec 11, 2000

$<$ EMI - ElectroMagnetic Instruments 


\section{ROTATION ANGLE}

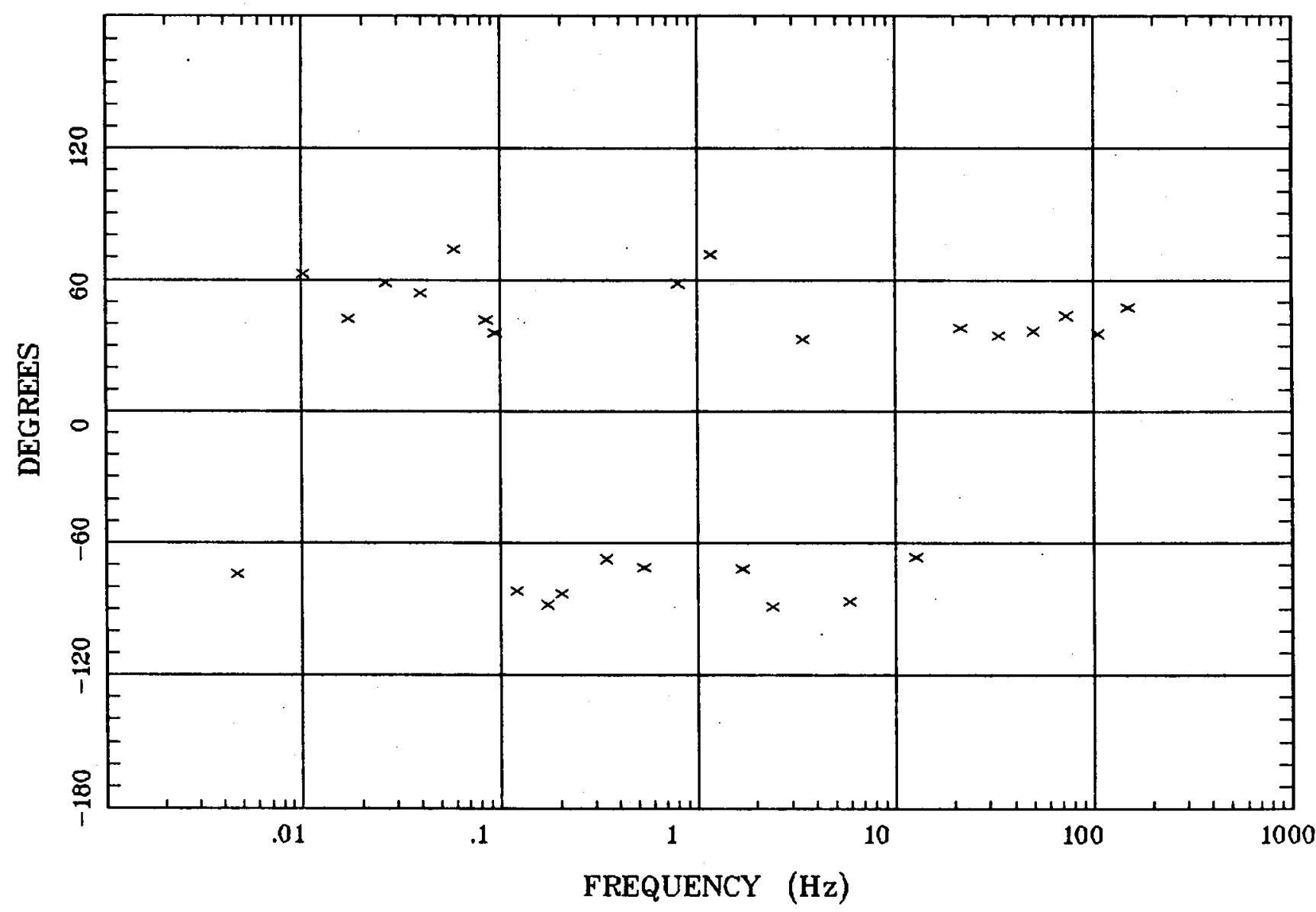

Client:

Remote:

Acquired:

Survey Co:
Rotation:

Filename: CT24C

Channels: Ch1 Ch2 Ch3 Ch4 Ch5 Ch6 Ch7

Plotted: 15:57 Dec 11, 2000

< EMI - ElectroMagnetic Instruments > 


\section{IMPEDANCE SKEW}

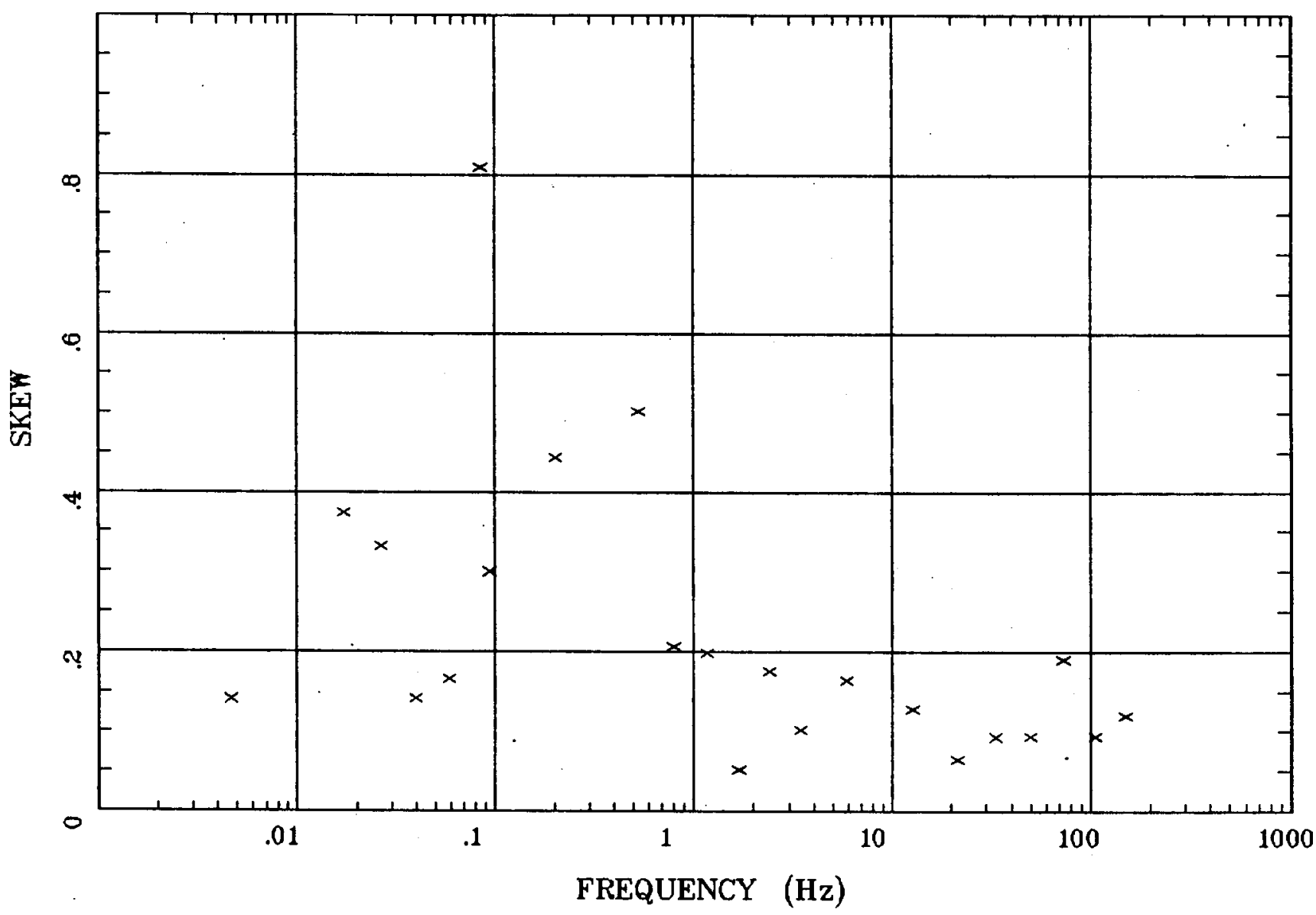

Client:

Remote:

Acquired:

Survey Co:
Rotation:

Filename: CT24C

Channels: Ch1 Ch2 Ch3 Ch4 Ch5 Ch6 Ch7

Plotted: $15: 57$ Dec 1.1, 2000

< EMI - ElectroMagnetic Instruments > 
E MULT Coh.

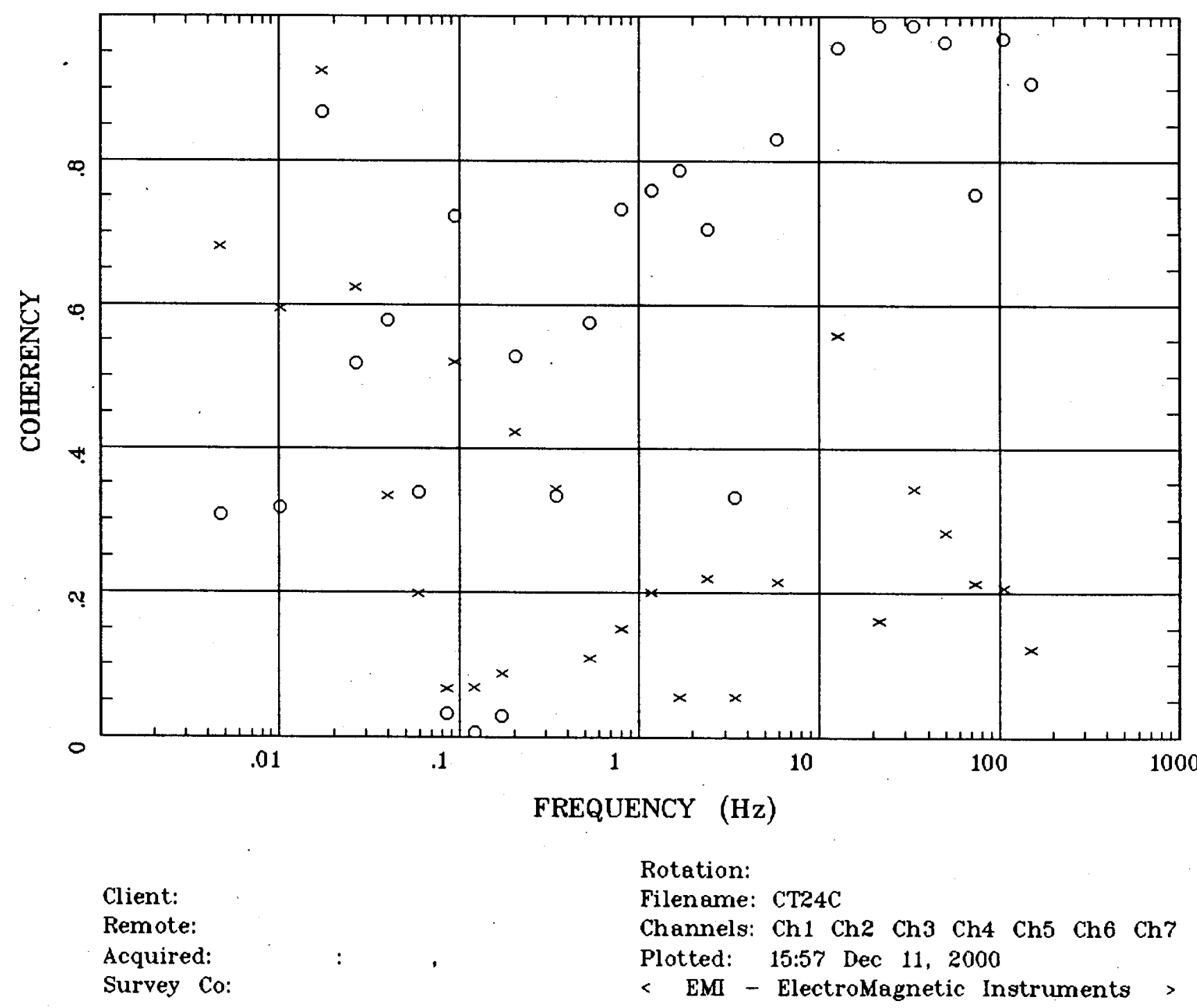




\section{POLAR PLOTS}

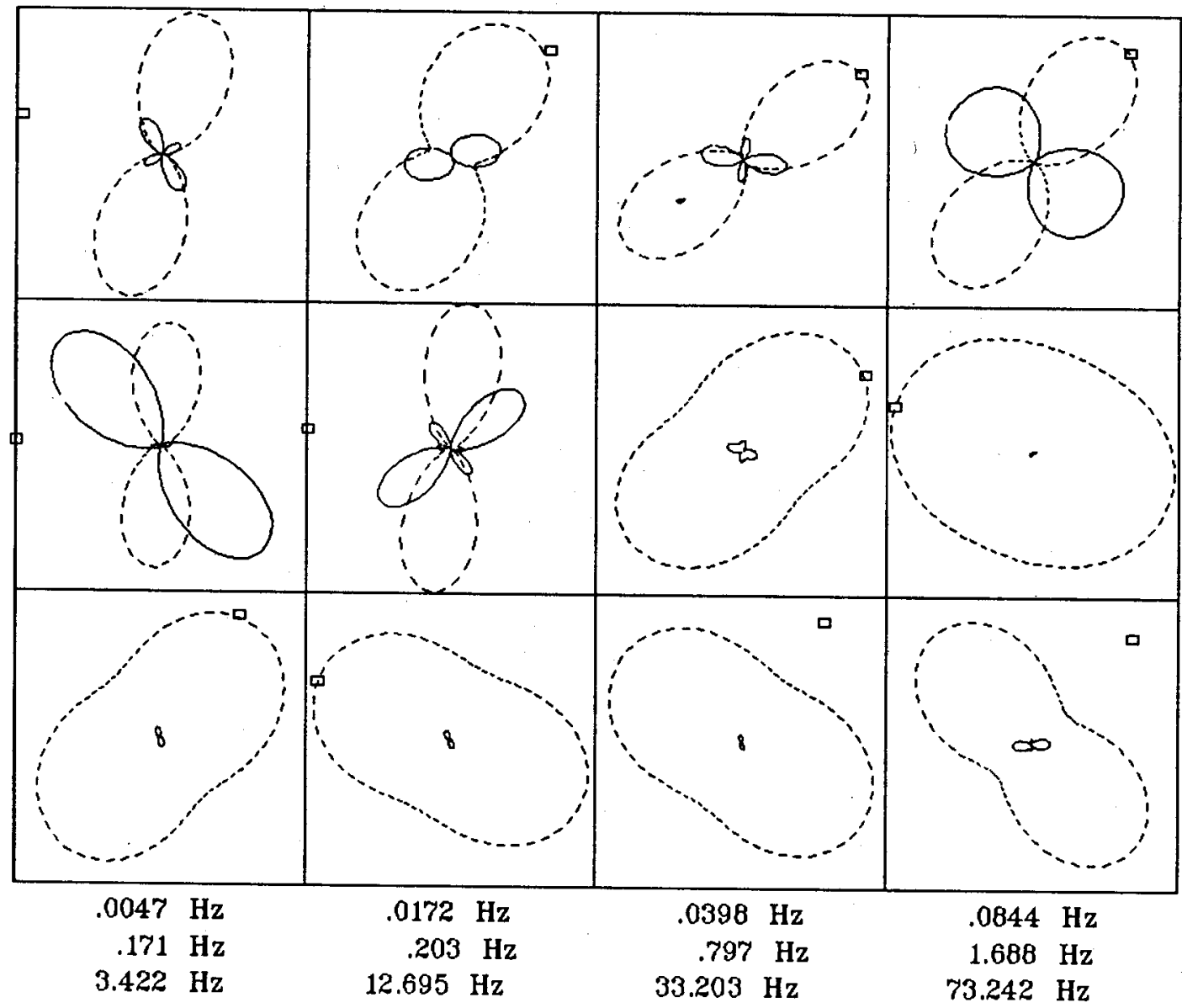

Client:

Remote:

Acquired:

Survey Co:
Rotation:

Filename: CT24C

Channels: Ch1 Ch2 Ch3 Ch4 Ch5 Ch6 Ch7 Plotted: 15:57 Dec 11, 2000

$<$ EMI - ElectroMagnetic Instruments > 


\section{APPARENT RESISTIVITY}

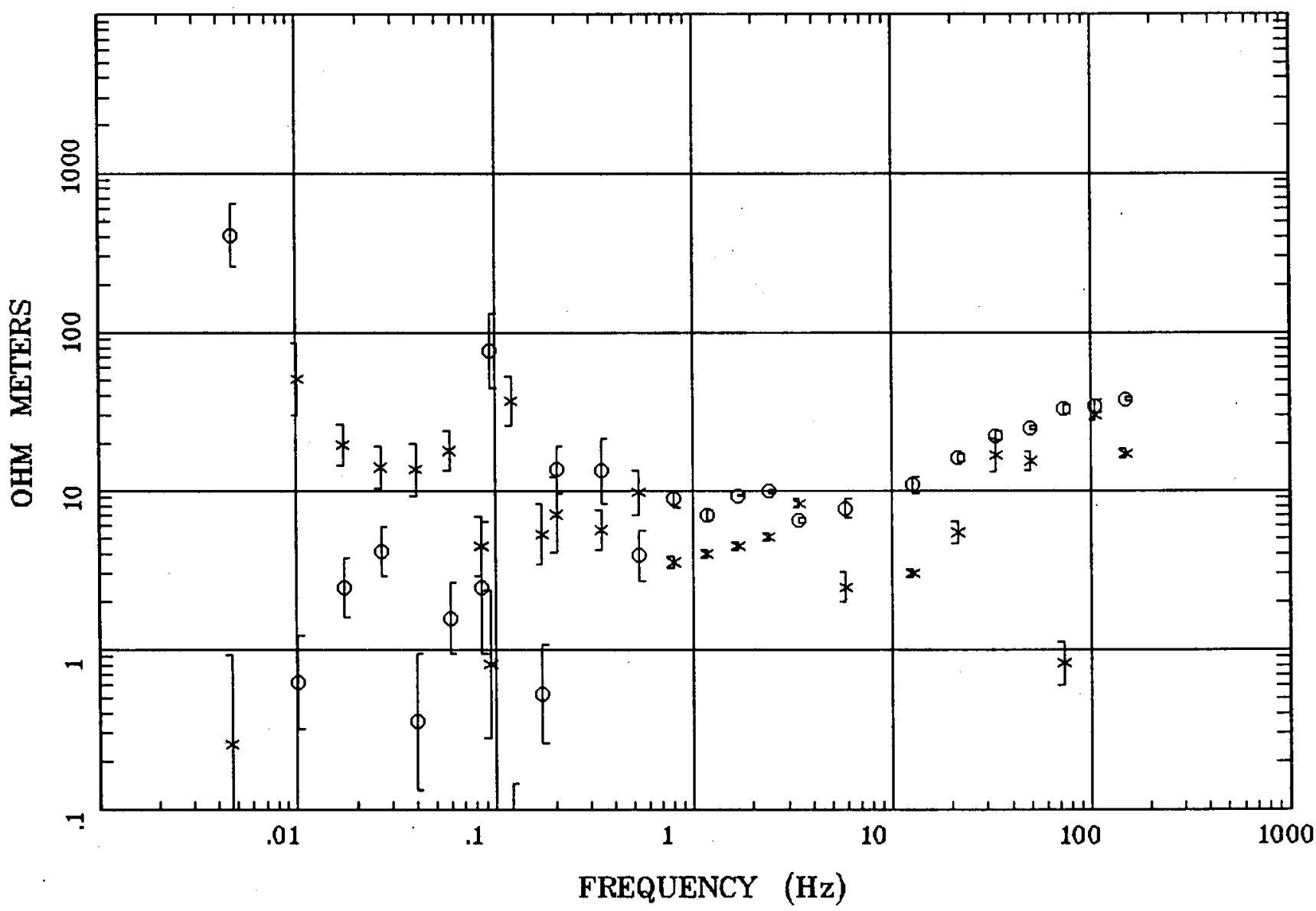

Client:

Remote:

Acquired: Survey Co:
Rotation:

Filename: CT25D

Channels: Ch1 Ch2 Ch3 Ch4 Ch5 Ch6 Ch7 Platted: 15:58 Dec 11, 2000

< EMI - ElectroMagnetic Instruments 
Station 25B

IMPEDANCE PHASE

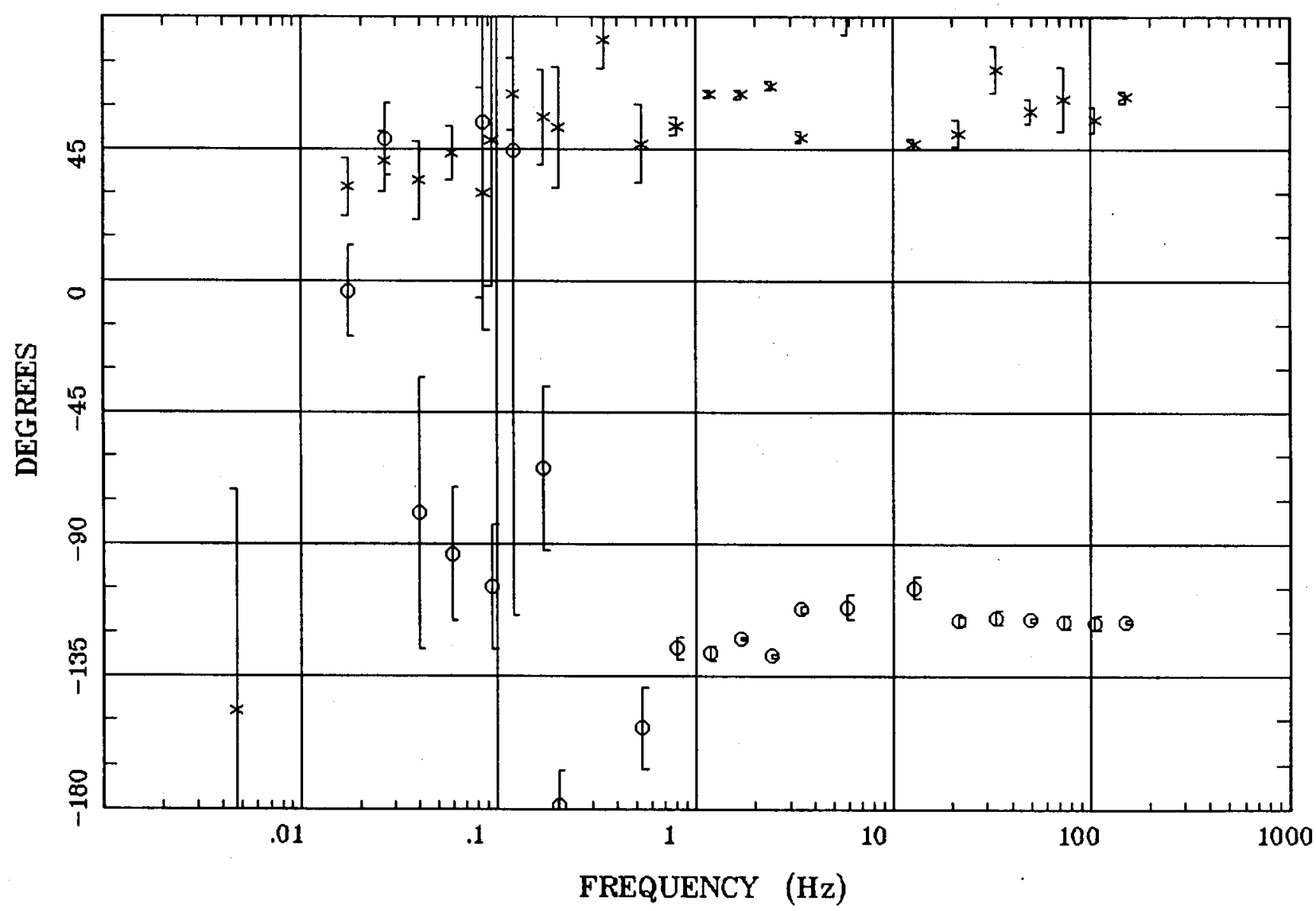

Client:

Rotation:

Filename: CT25D

Remote:

Filename: CT25D

Channels: Ch1 Ch2 Ch3 Ch4 Ch5 Ch6 Ch7

Acquired:

Plotted: 15:58 Dec 11, 2000

Survey Ca:

$<$ EMI - ElectroMagnetic Instruments 


\section{ROTATION ANGLE}

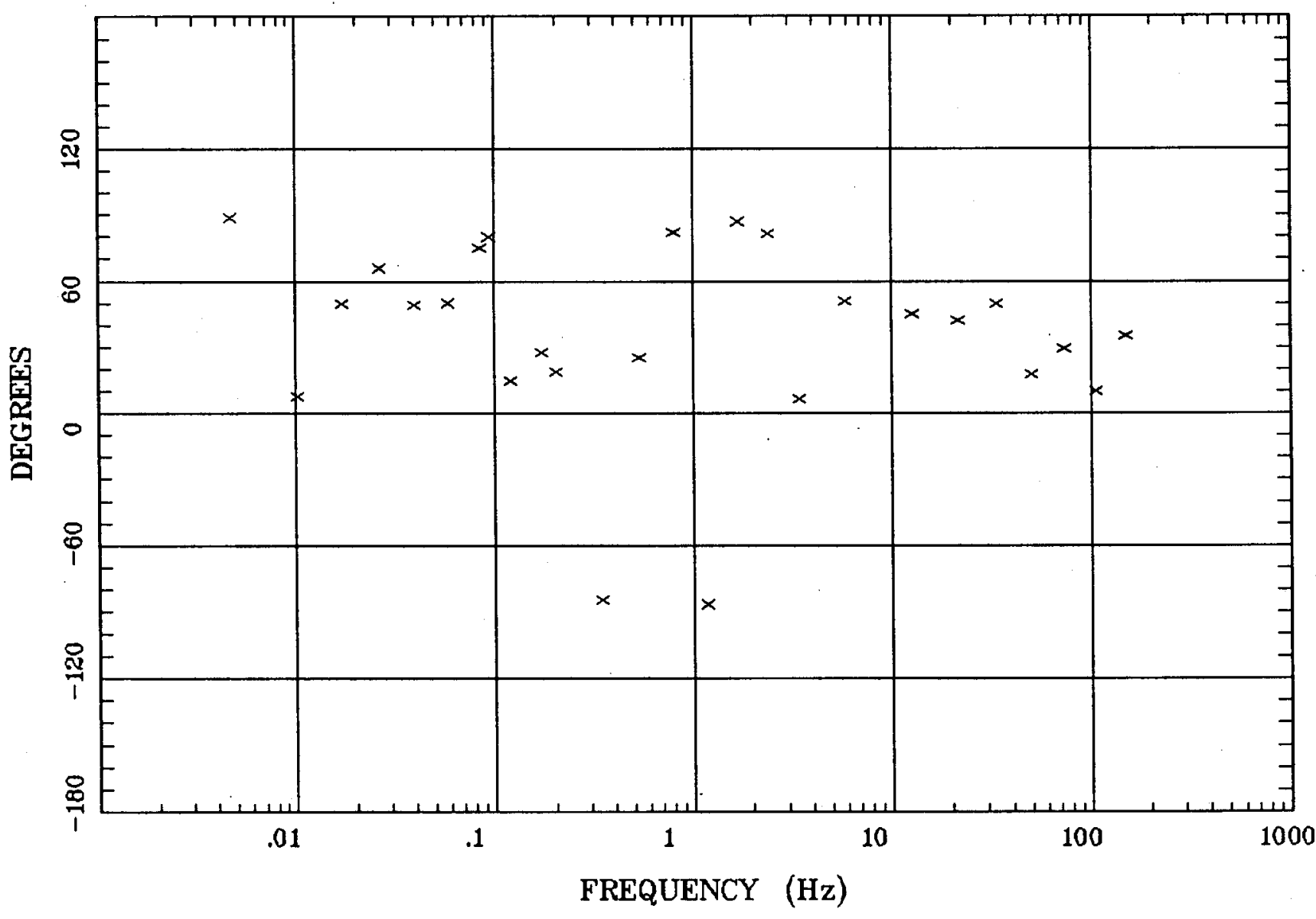

Client:

Remote:

Acquired: Survey Co:
Rotation:

Filename: CT25D

Channels: Ch1 Ch2 Ch3 Ch4 Ch5 Ch6 Ch7

Platted: 15:58 Dec 11, 2000

< EMI - ElectroMagnetic Instruments 
Station 25B

IMPEDANCE SKEW

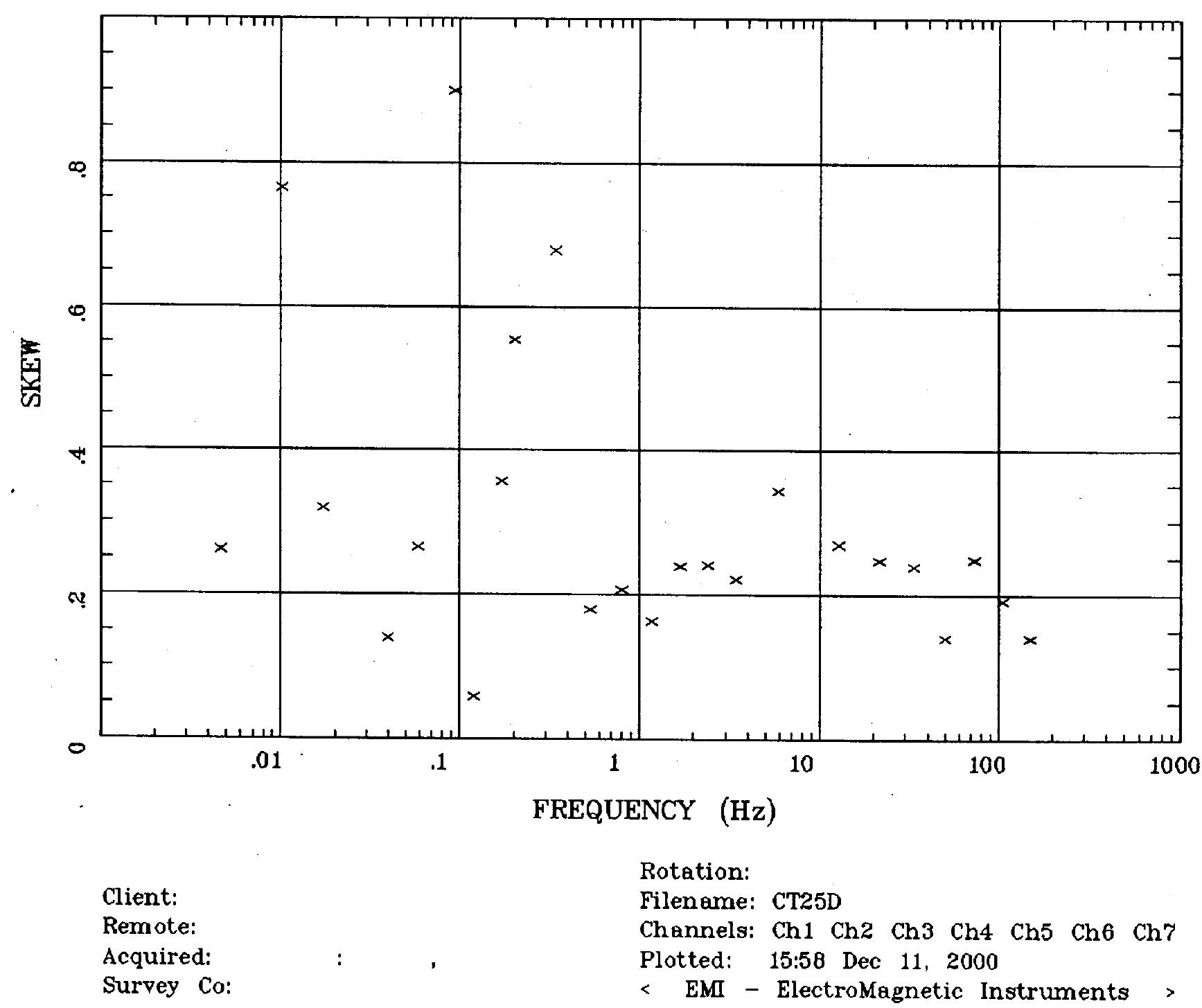


E MULT Coh.

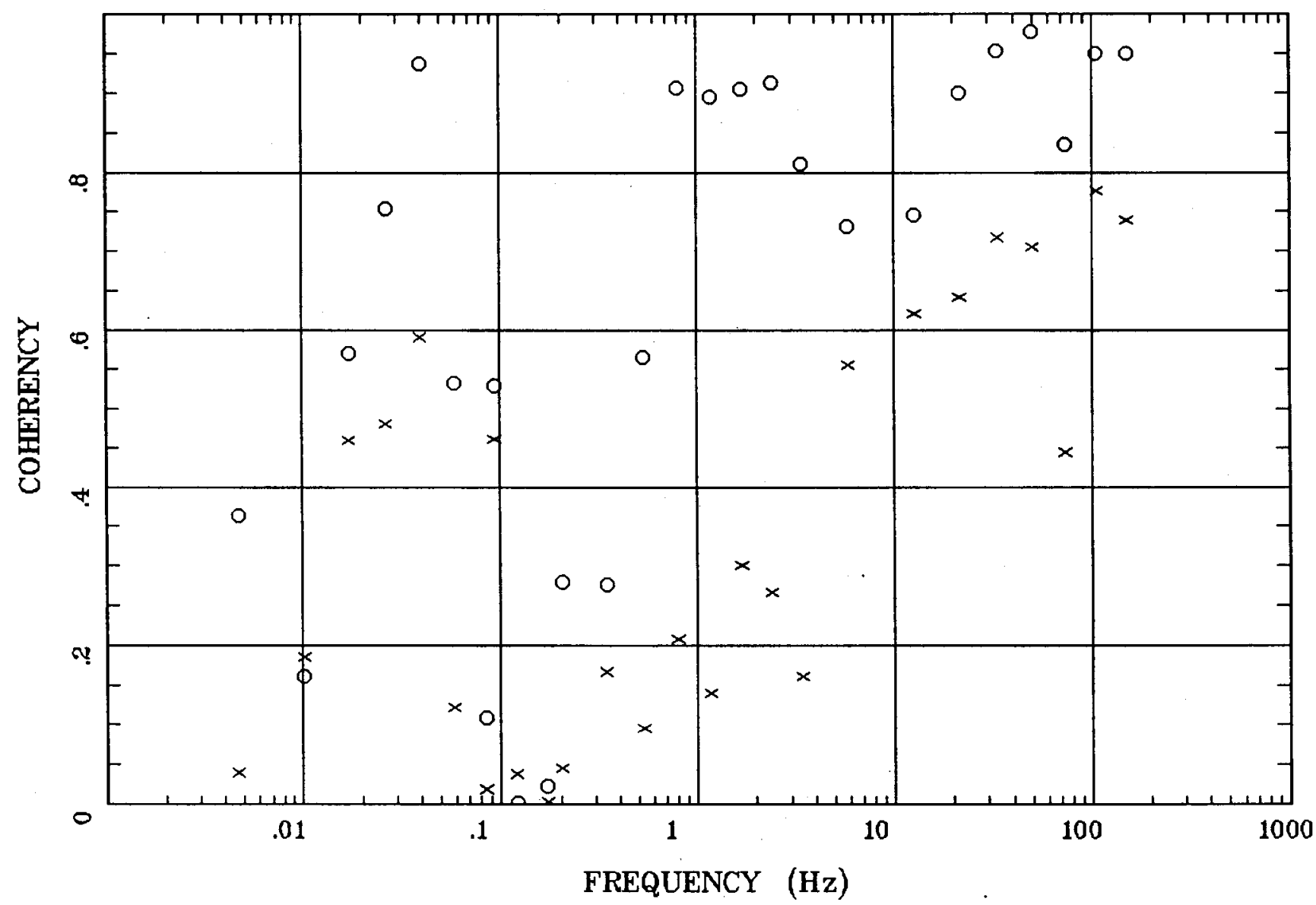

Client:

Remote:

Acquired:

Survey $\mathrm{Co}$
Rotation:

Filename: CT25D

Channels: Ch1 Ch2 Ch3 Ch4 Ch5 Ch6 Ch7

Plotted: 15:58 Dec 11, 2000

$<$ EMI - ElectroMagnetic Instruments 


\section{POLAR PLOTS}

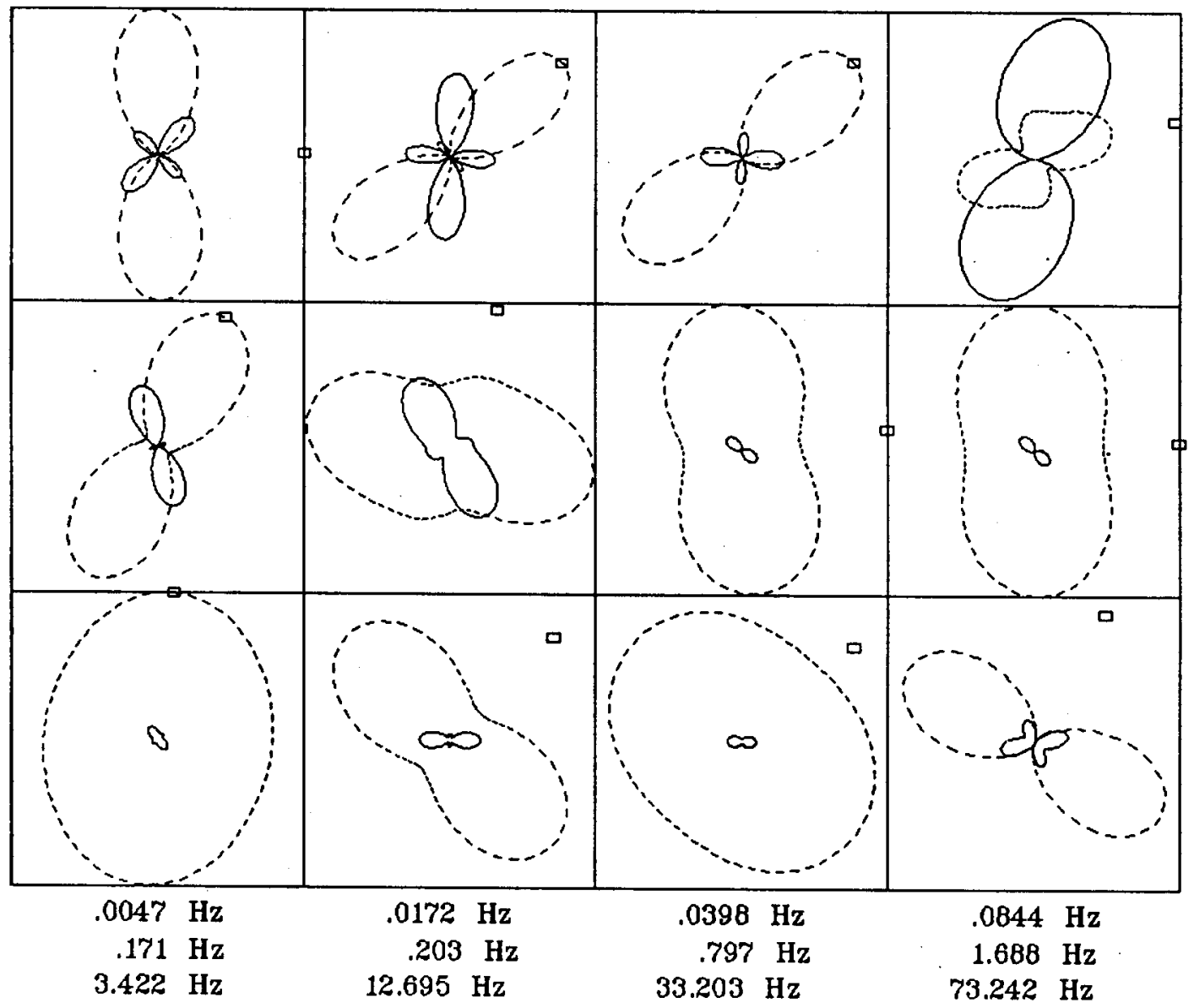

Client:

Remote:

Acquired:

Survey Co:
Rotation:

Filename: CT25D

Channels: Ch1 Ch2 Ch3 Ch4 Ch5 Ch6 Ch7

Plotted: 15:58 Dec 11, 2000

< EMI - ElectroMagnetic Instruments 


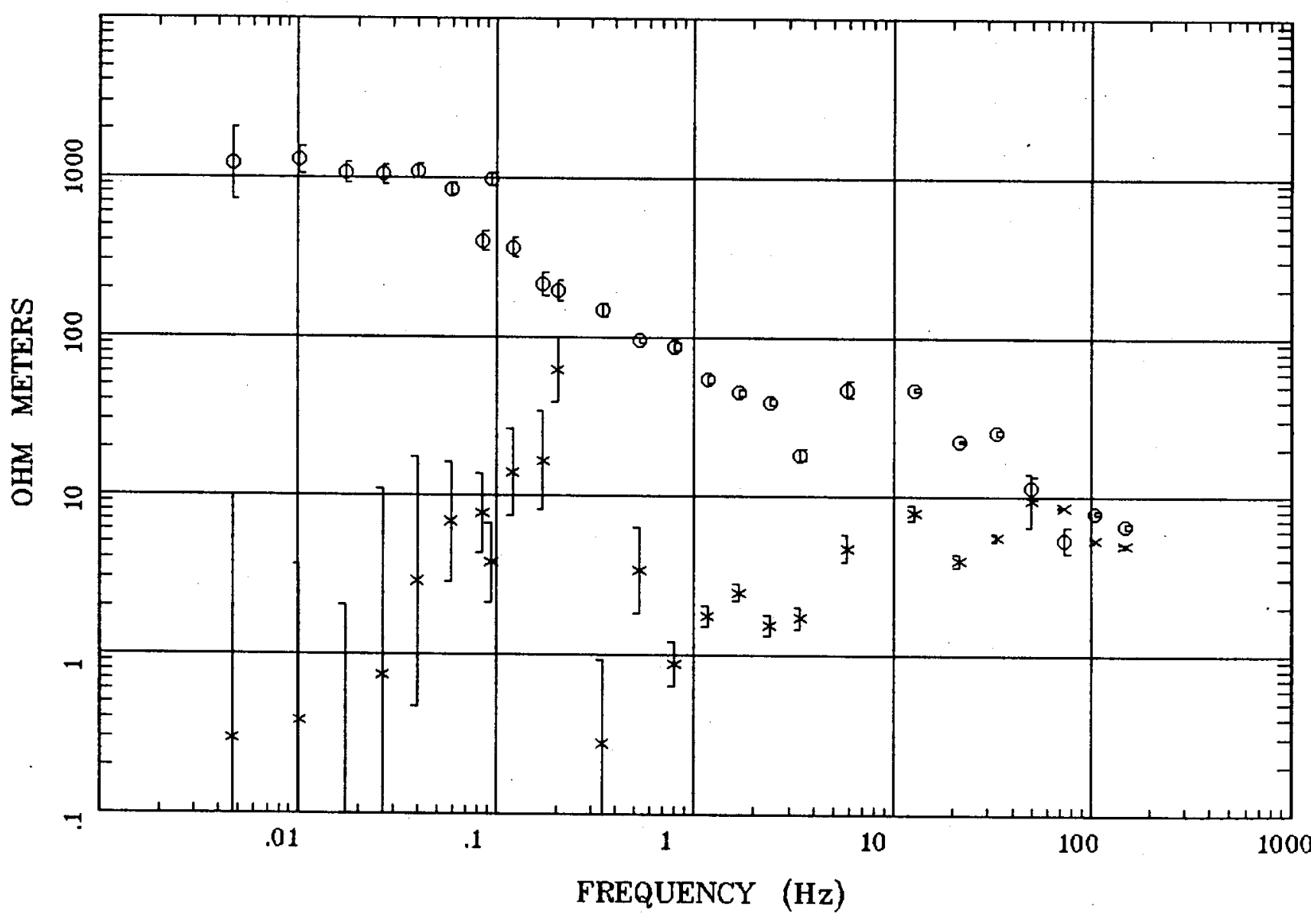

Client:

Remate:

Acquired:

Survey Co:
Rotation:

Filename: CT26C

Channels: Ch1 Ch2 Ch3 Ch4 Ch5 Ch6 Ch7 Plotted: 15:59 Dec 11, 2000

$<$ EMI - ElectroMagnetic Instruments 


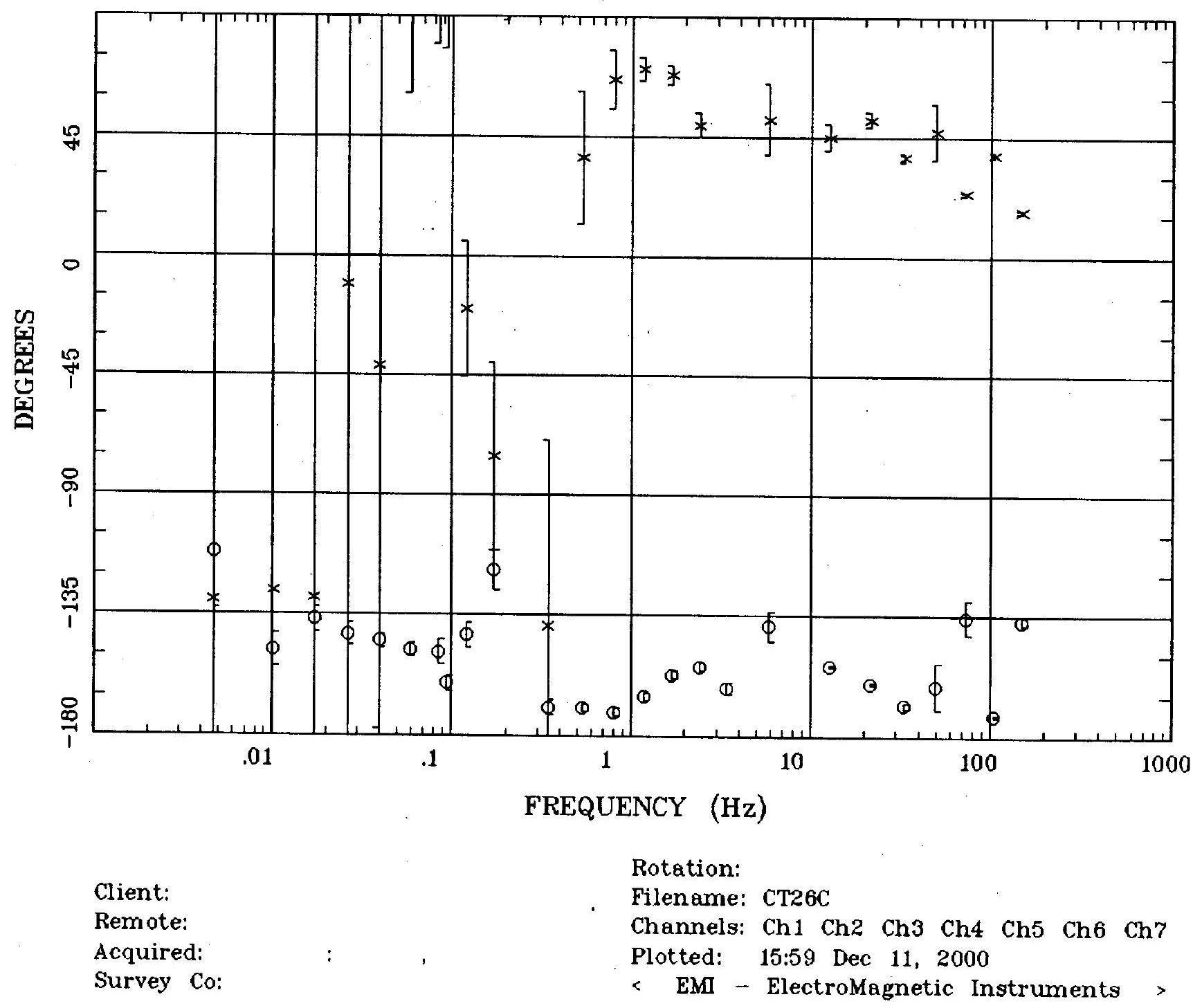




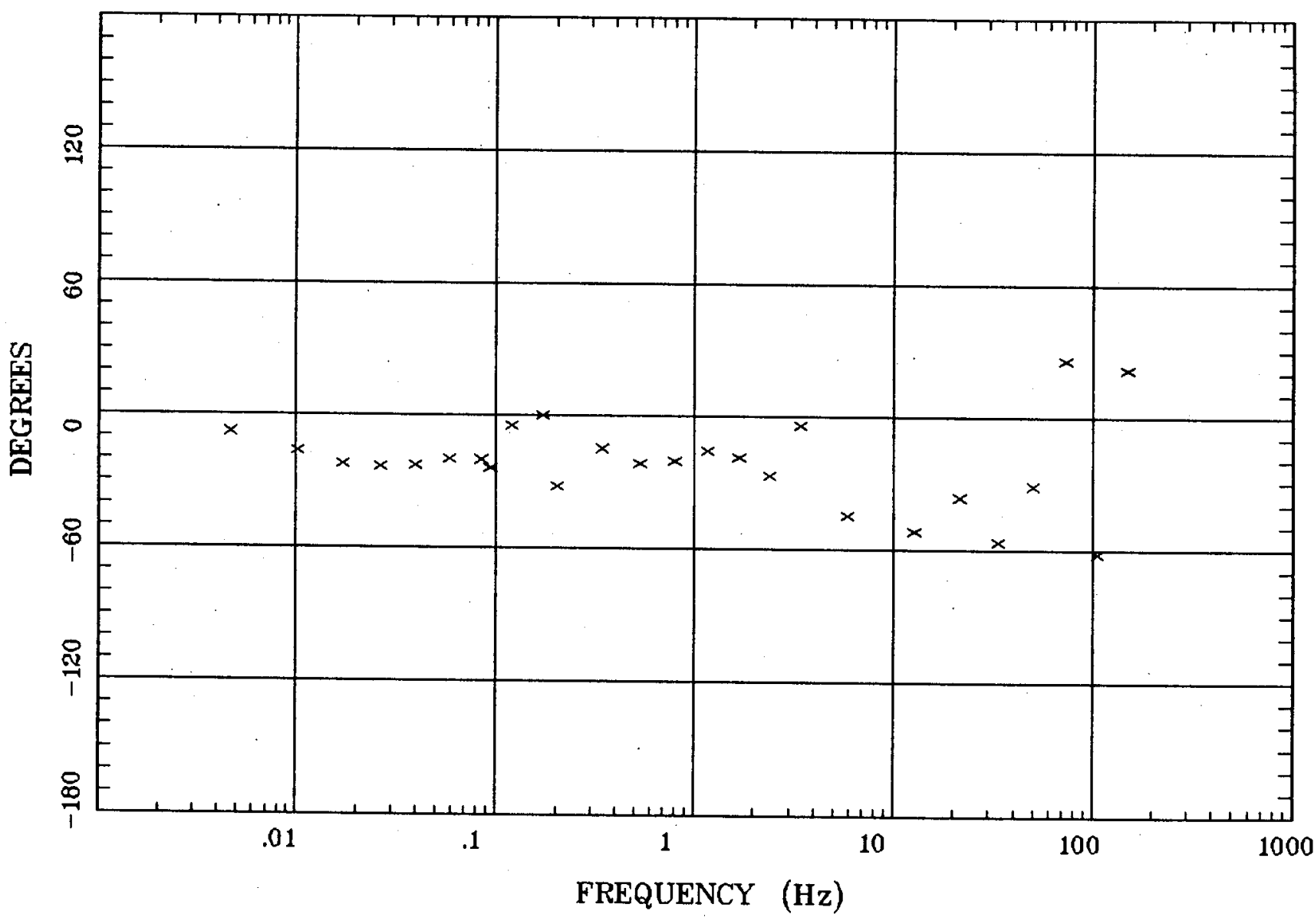

Client:

Remote:

Acquired:

Survey Co:
Rotation:

Filename: CT26C

Channels: Ch1 Ch2 Ch3 Ch4 Ch5 Ch6 Ch7 Plotted: 15:59 Dec 11, 2000

< EMI - ElectroMagnetic Instruments 


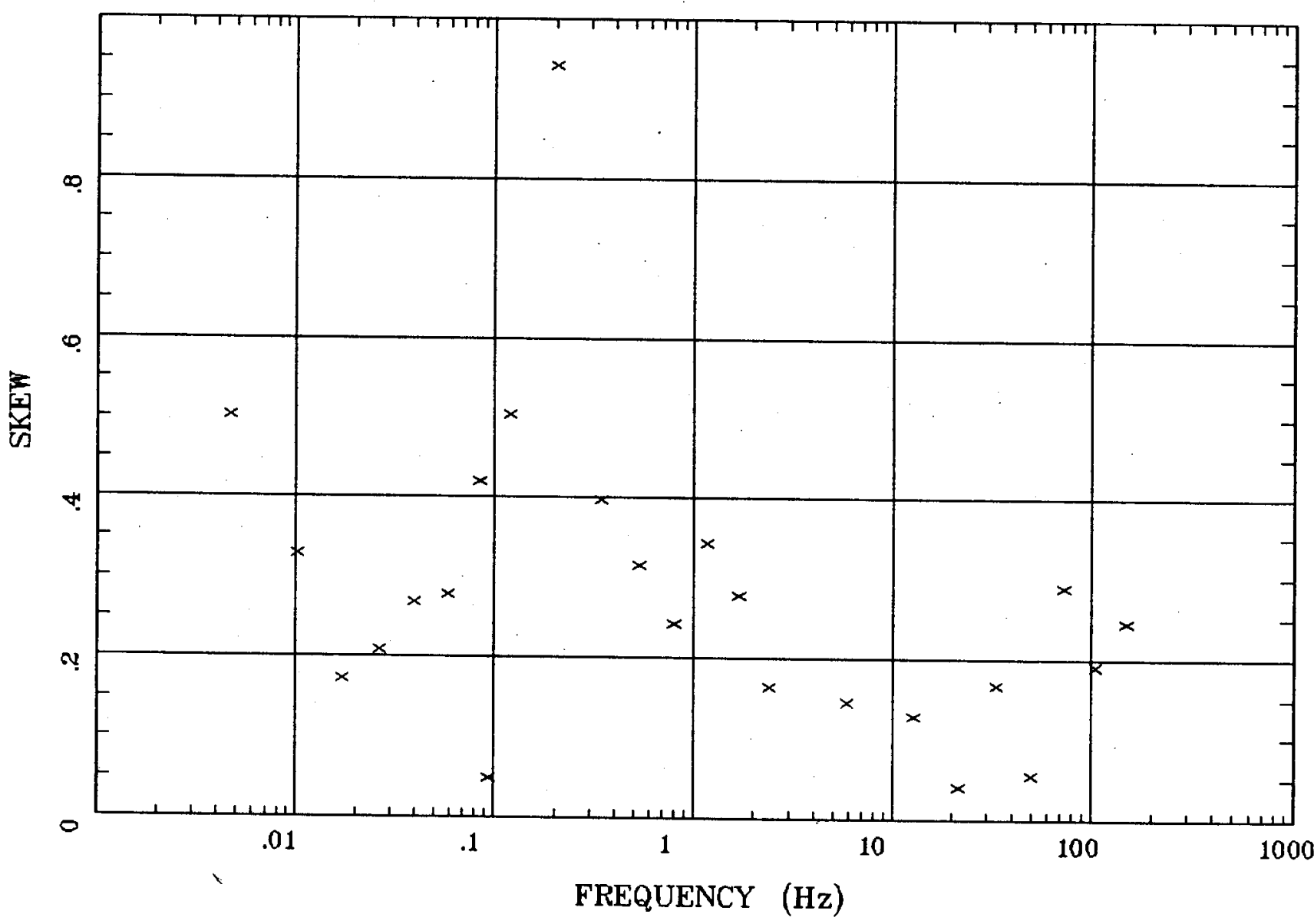

Client:

Remote:

Acquired:

Survey Co:
Rotation:

Filename: CT26C

Channels: Ch1 Ch2 Ch3 Ch4 Ch5 Ch6 Ch7

Plotted: 15:59 Dec 11, 2000

$<$ EMI - ElectroMagnetic Instruments 


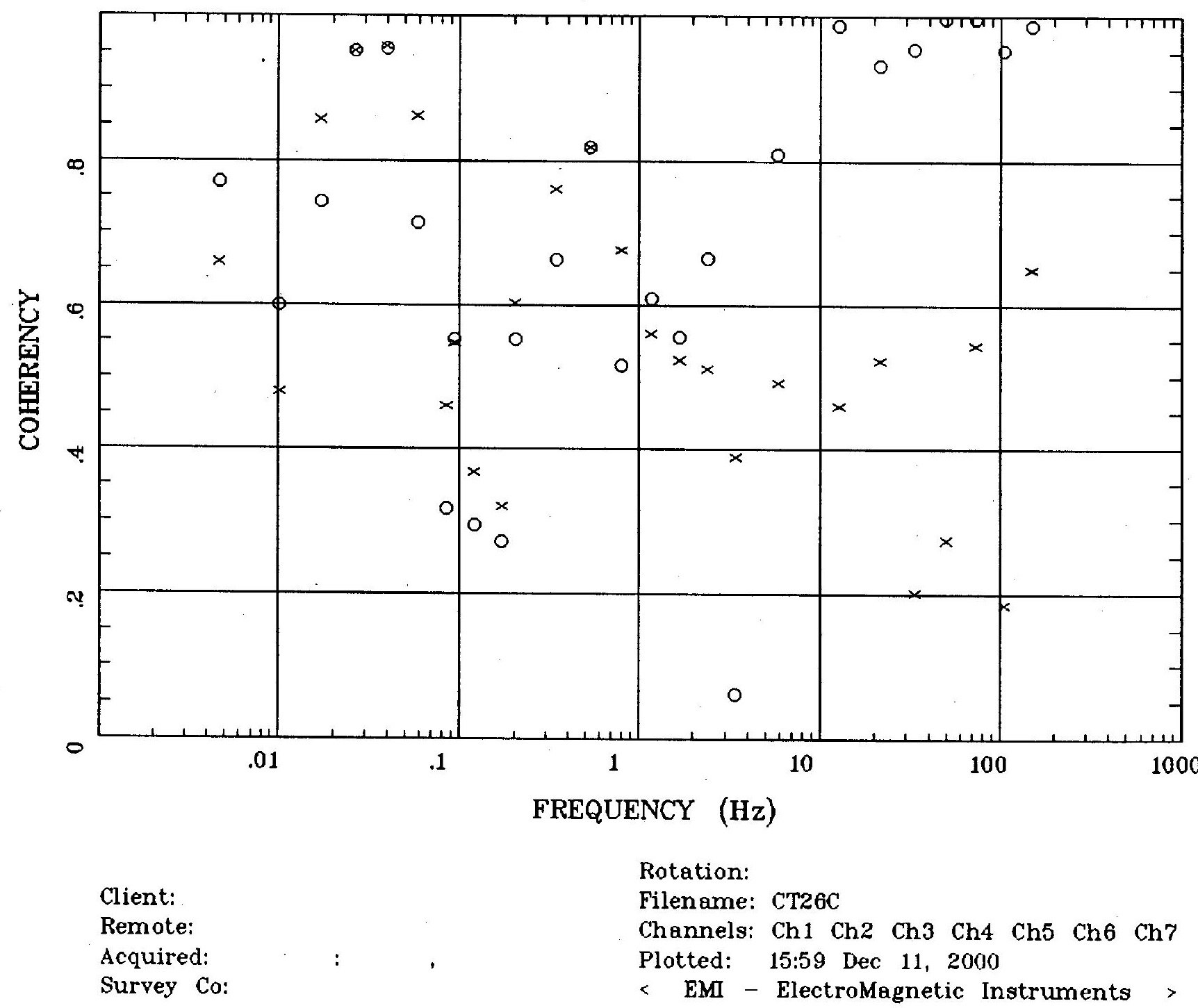




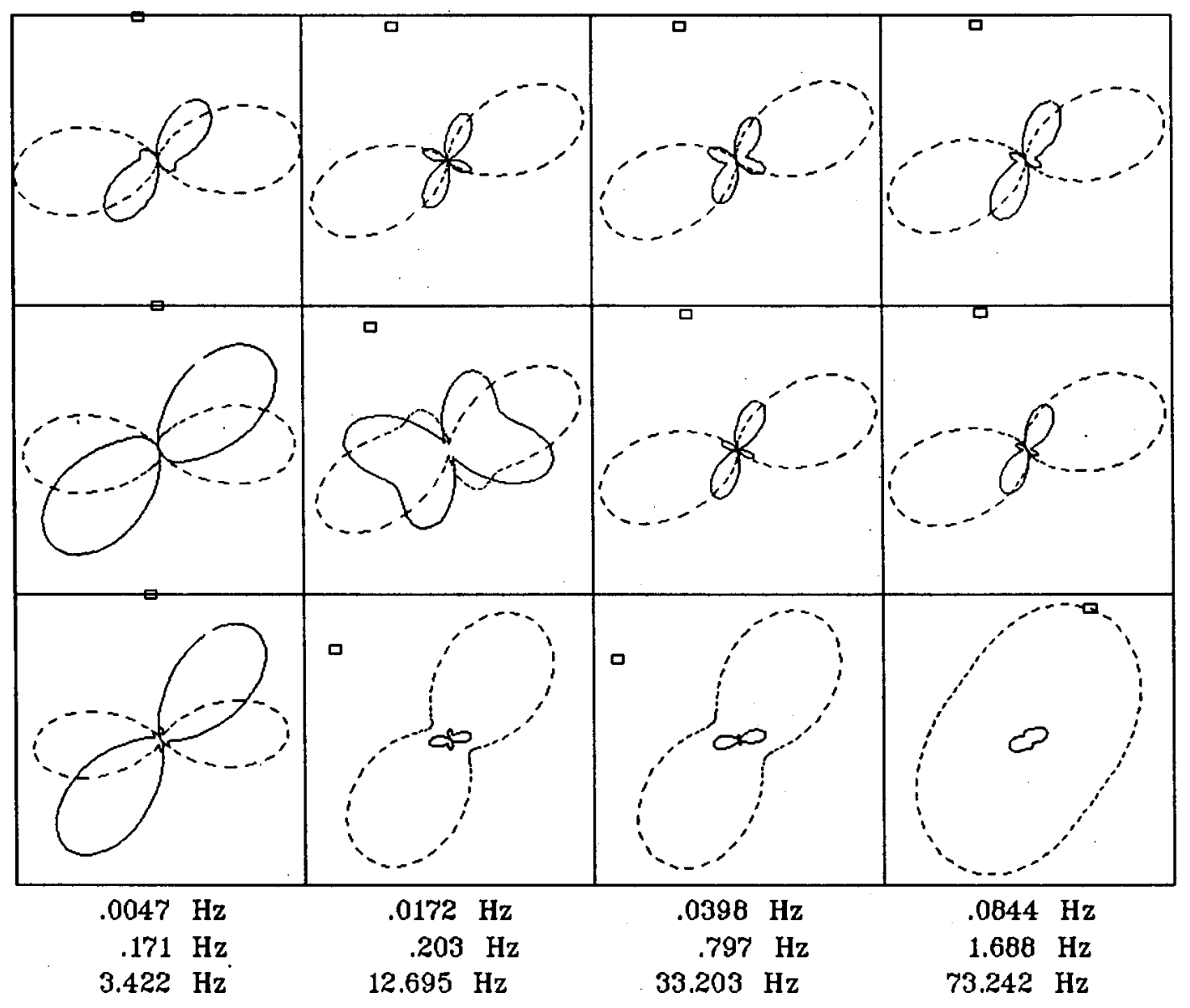

Rotation:

Client:

Remote:

Acquired:

Survey Co:
Filename: CT26C

Channels: Ch1 Ch2 Ch3 Ch4 Ch5 Ch6 Ch7

Plotted: 15:59 Dec 11, 2000

$<$ EMI - ElectroMagnetic Instruments 


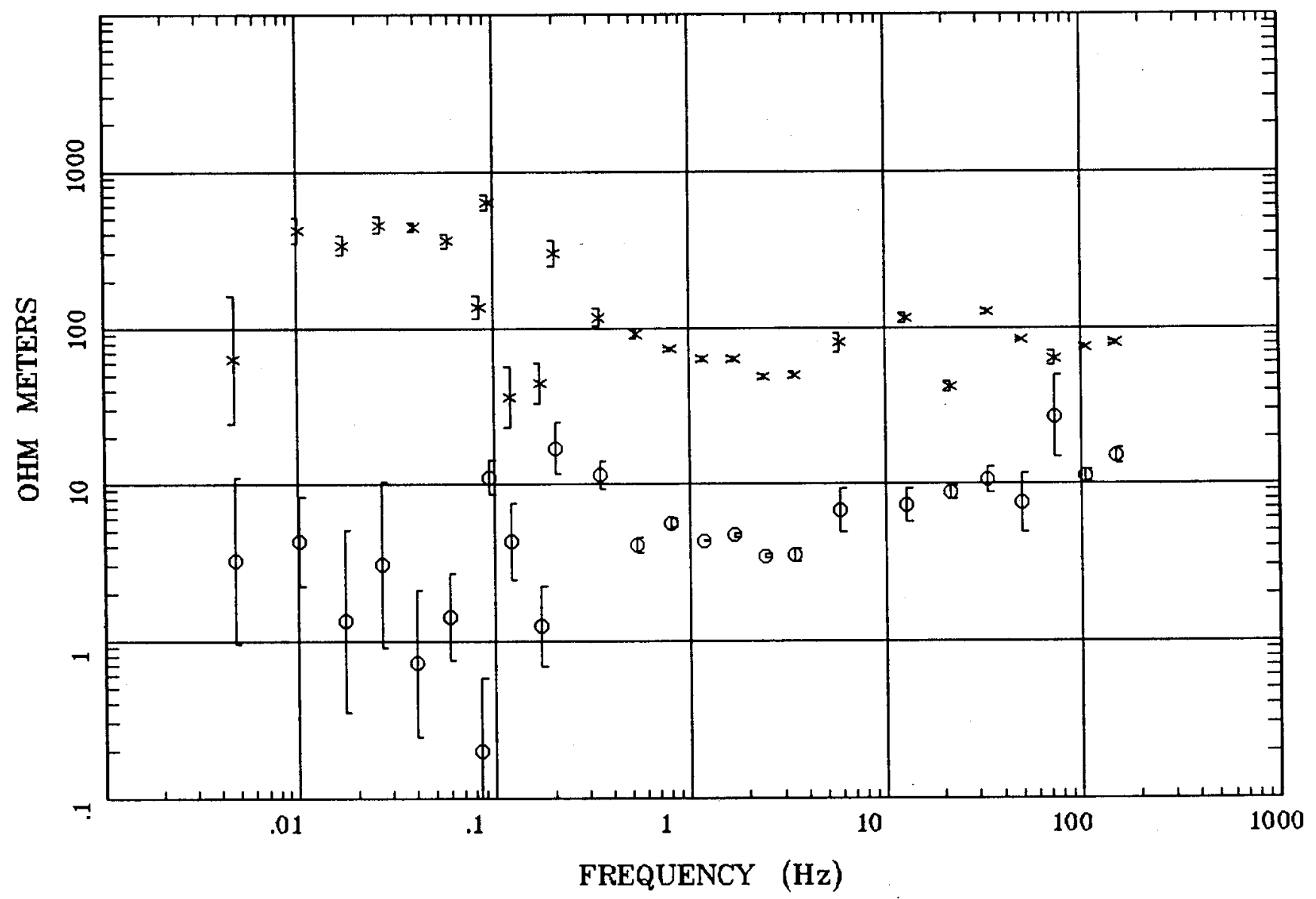

Client:

Remote: Acquired: Survey Co:
Rotation:

Filename: CT27A

Channels: Ch1 Ch2 Ch3 Ch4 Ch5 Ch6 Ch7 Plotted: 16:00 Dec 11, 2000

< EMI - ElectroMagnetic Instruments > 


\section{IMPEDANCE PHASE}

Station 27B

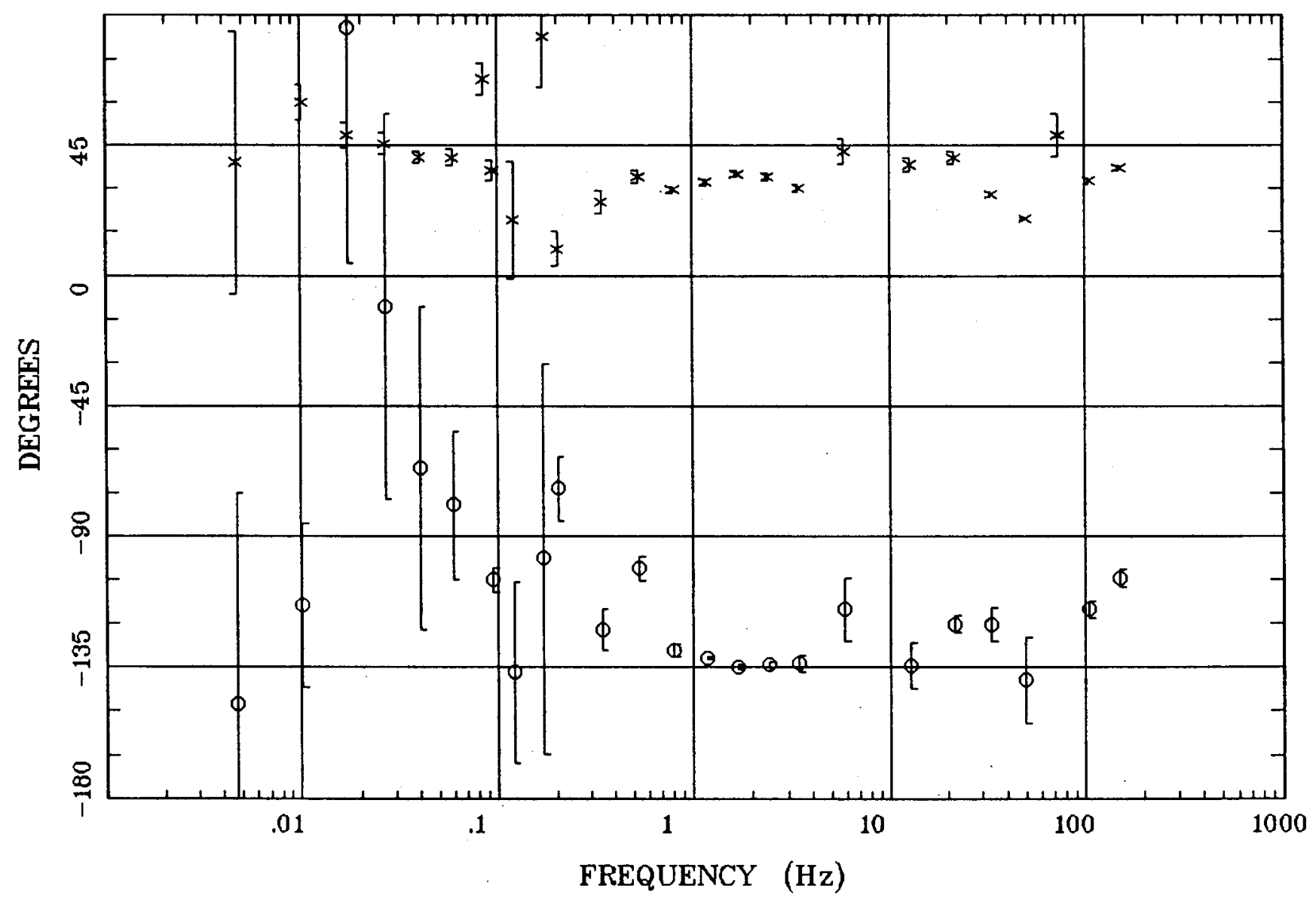

Client:

Remote:

Acquired

Survey Co:
Rotation:

Filename: CT27A

Channels: Ch1 Ch2 Ch3 Ch4 Ch5 Ch6 Ch7

Plotted: 16:00 Dec 11, 2000

< EMI - ElectroMagnetic Instruments > 


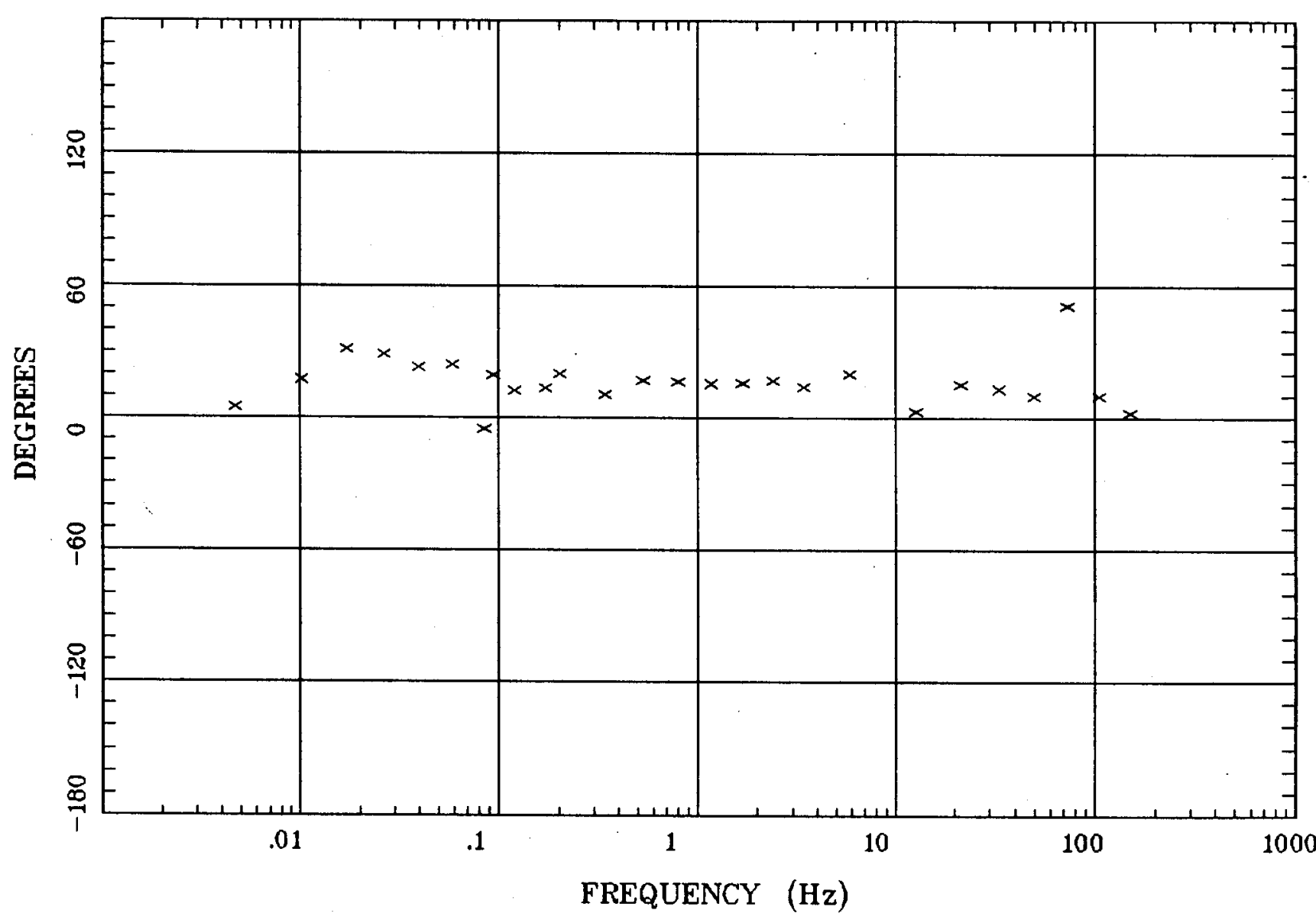

Client:

Remote:

Acquired:

Survey Co:
Rotation:

Filename: CT27A

Channels: Ch1 Ch2 Ch3 Ch4 Ch5 Ch6 Ch7

Platted: 16:00 Dec 11, 2000

< EMI - ElectroMagnetic Instruments $>$ 


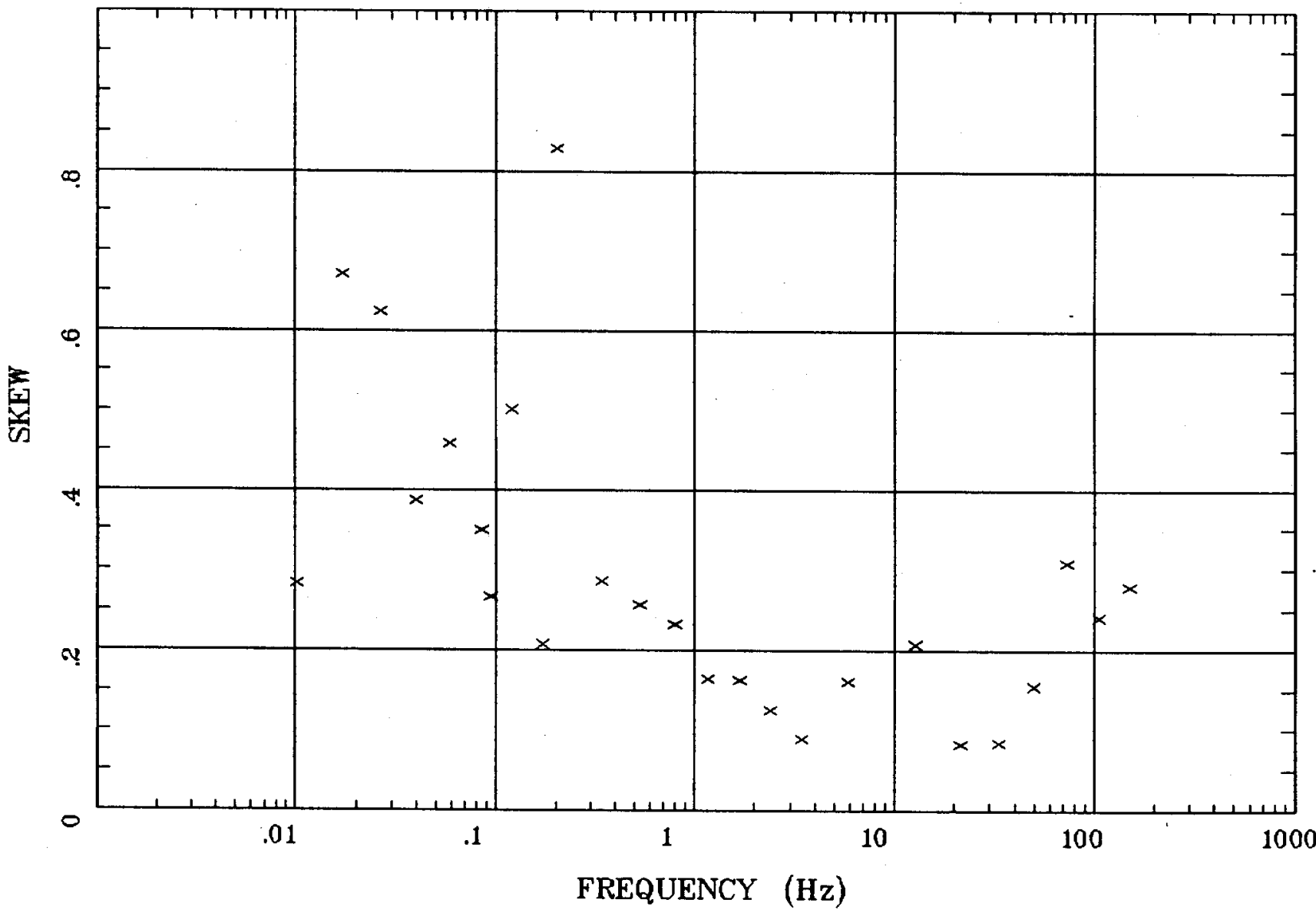

Client:

Remote:

Acquired:

Survey Co:
Rotation:

Filename: CT27A

Channels: Ch1 Ch2 Ch3 Ch4 Ch5 Ch6 Ch7

Plotted: 16:00 Dec 11, 2000

$<$ EMI - ElectroMagnetic Instruments 


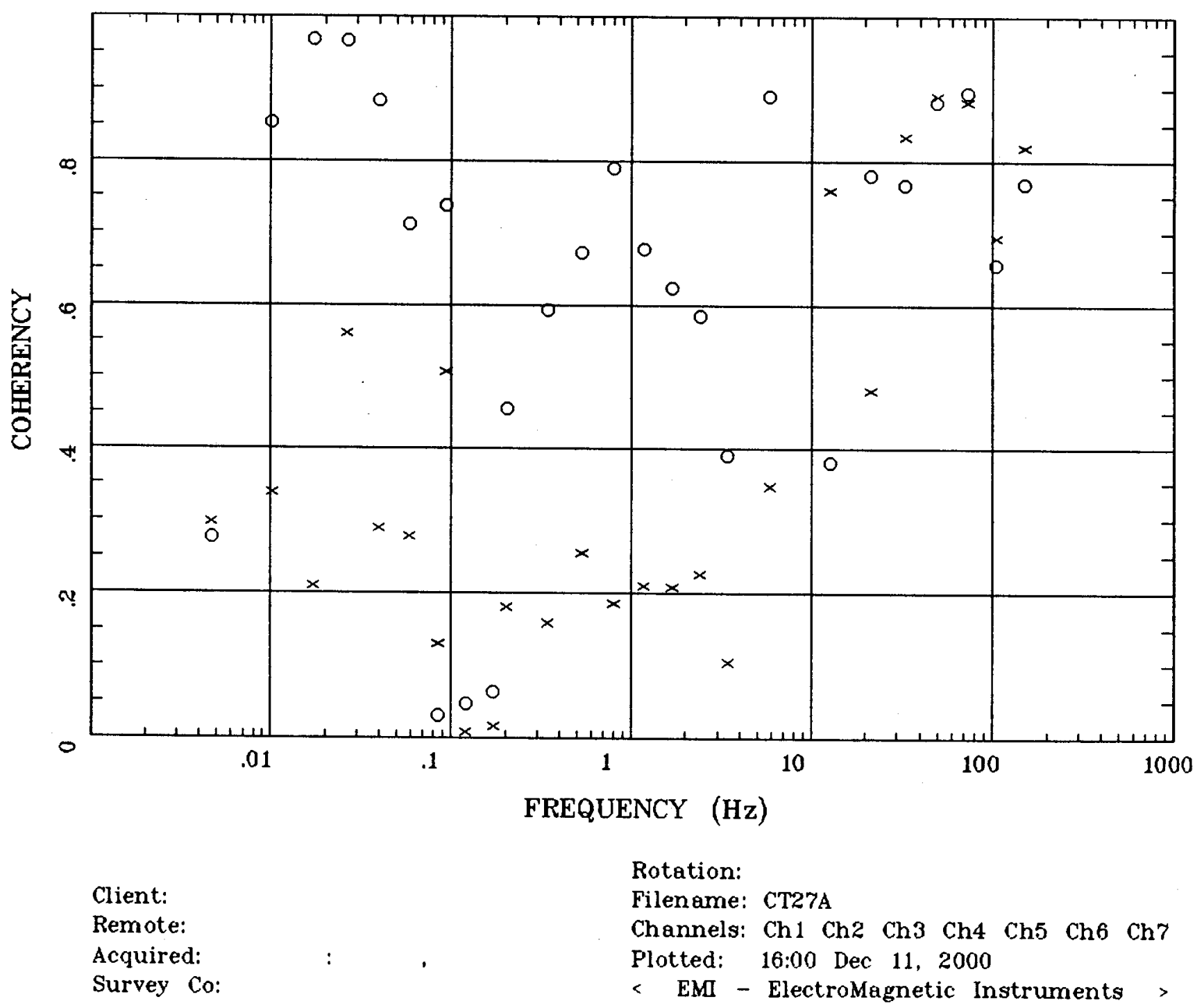




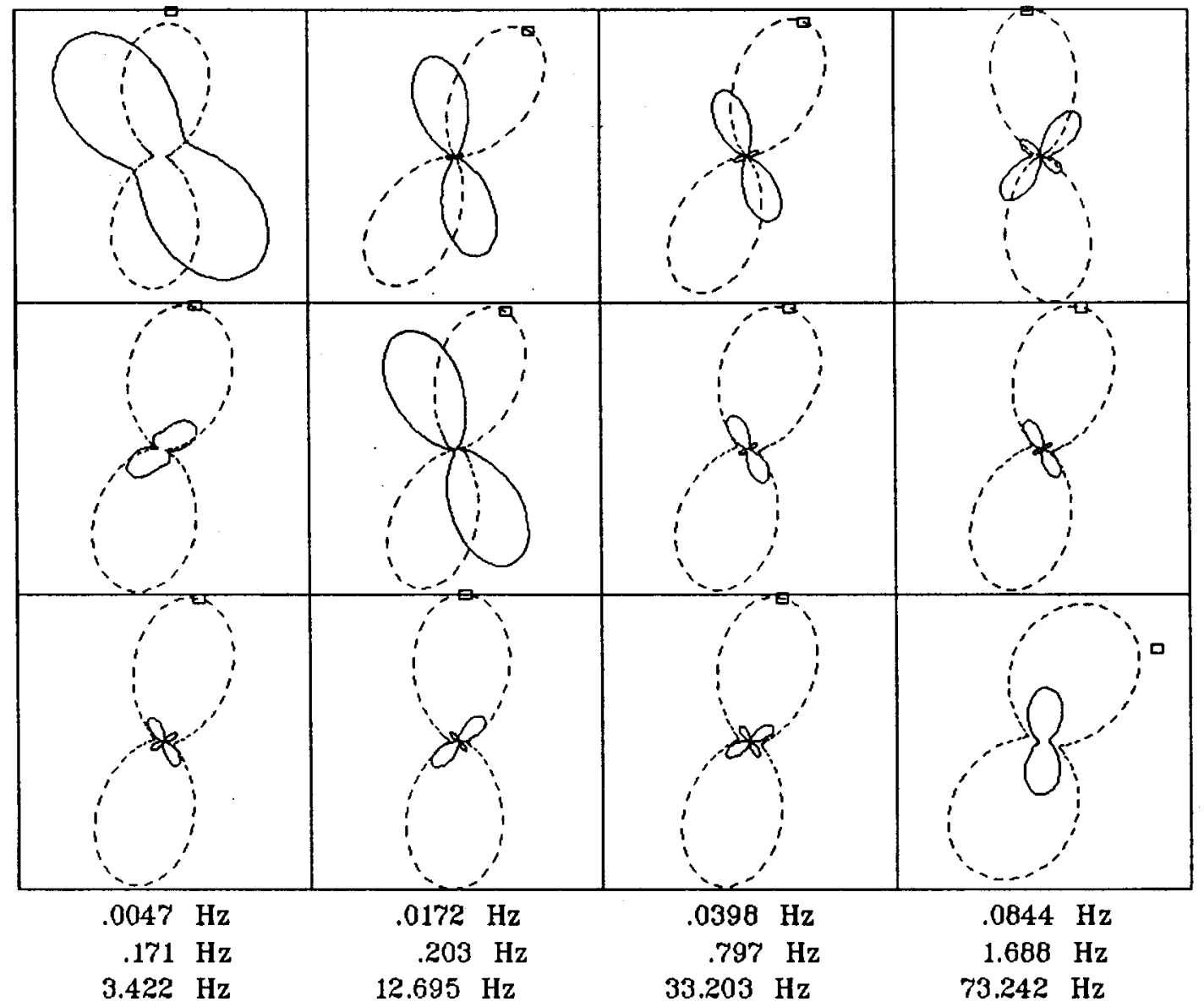

Client:

Remote:

Acquired:

Survey Co:

\section{Rotation:}

Filename: CT27A

Channels: Ch1 Ch2 Ch3 Ch4 Ch5 Ch6 Ch7 Plotted: 16:00 Dec 11, 2000

< EMI - ElectroMagnetic Instruments > 


\section{APPARENT RESISTIVITY}

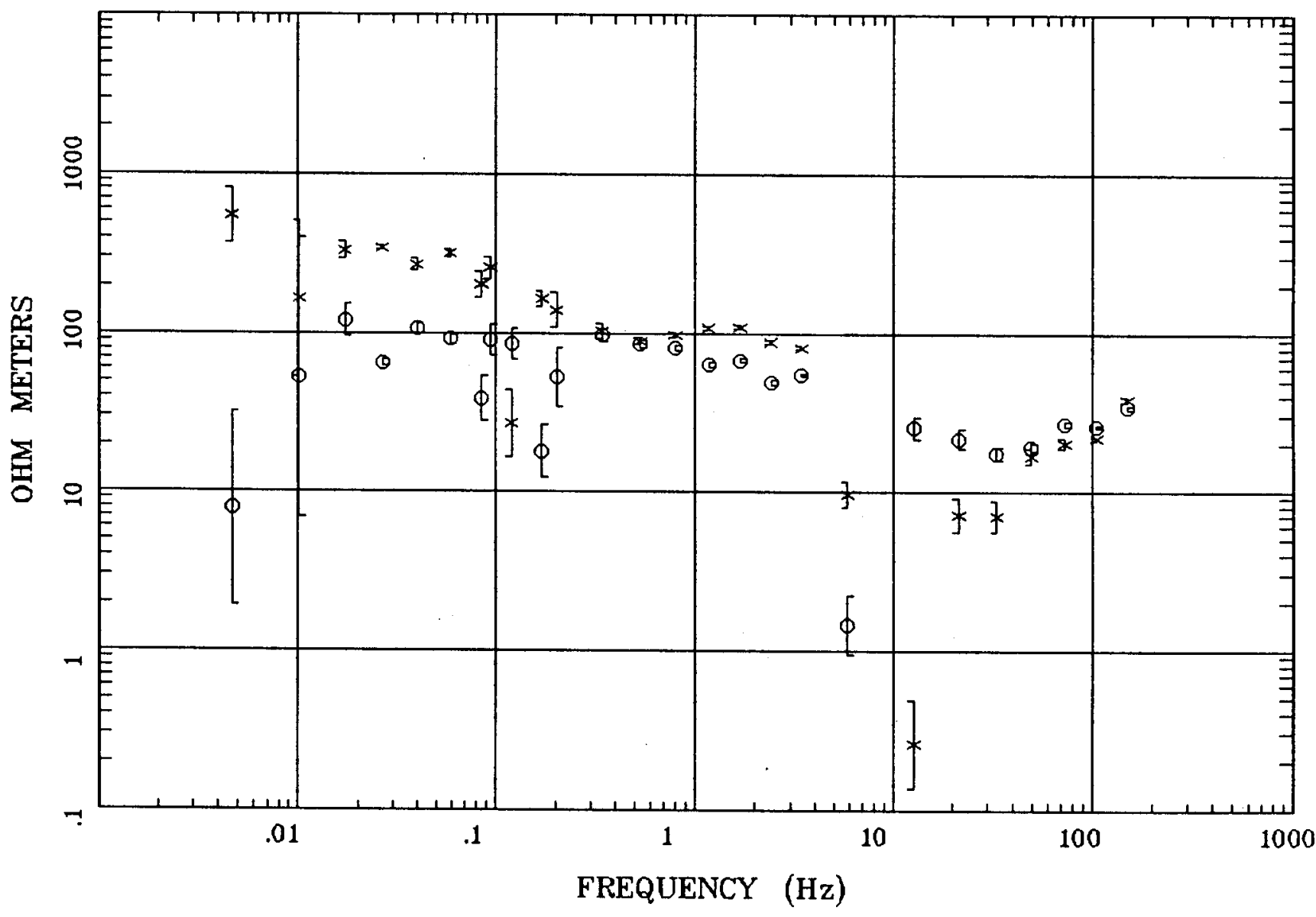

Client:

Remote:

Acquired:

Survey Co:
Rotation:

Filename: CT28C

Channels: Ch1 Ch2 Ch3 Ch4 Ch5 Ch6 Ch7

Plotted: 16:02 Dec 11, 2000

< EMI - ElectroMagnetic Instruments 


\section{Station 28B}

\section{IMPEDANCE PHASE}

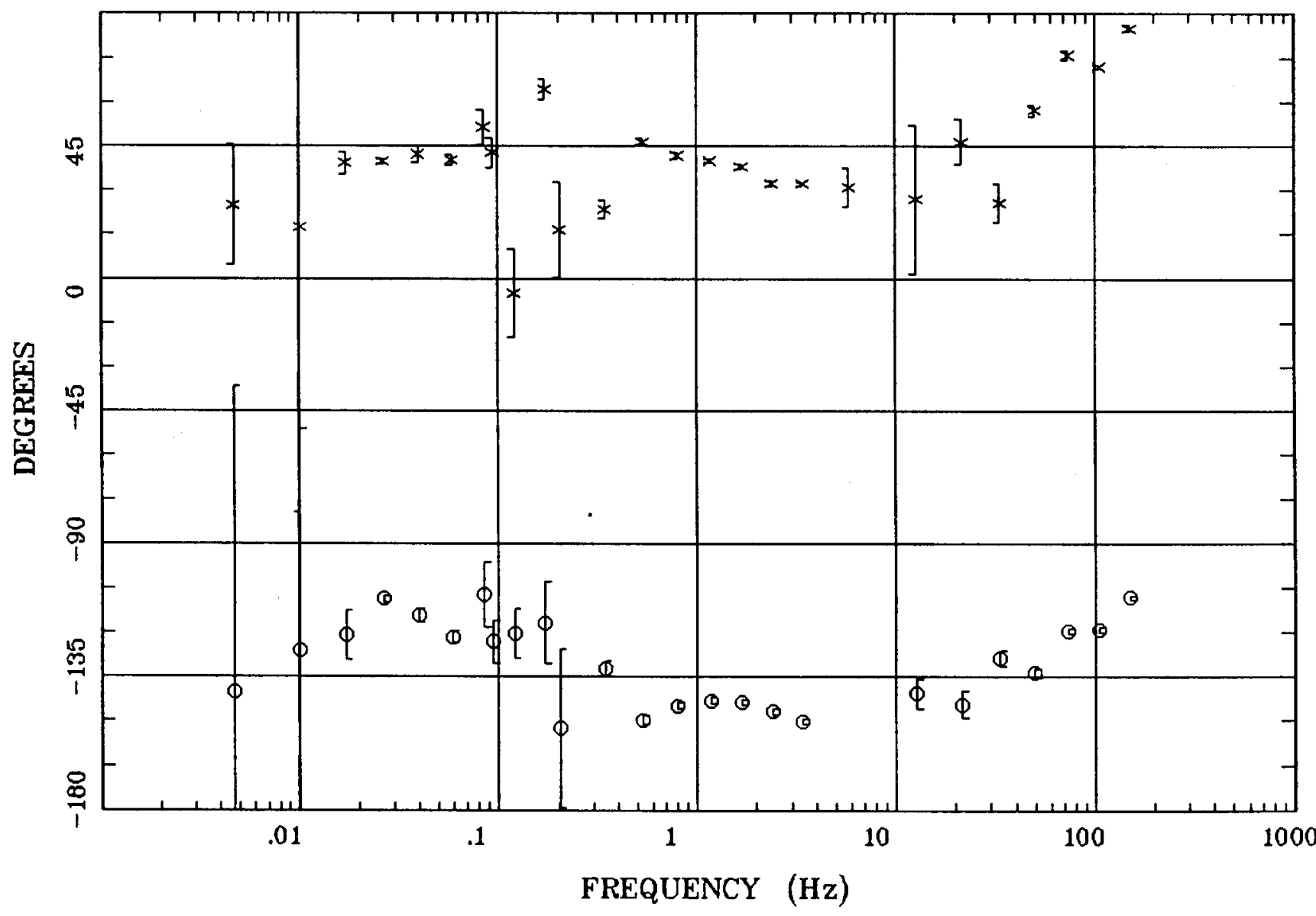

Client:

Remote:

Acquired:

Survey Co:
Rotation:

Filename: CT28C

Channels: Ch1 Ch2 Ch3 Ch4 Ch5 Ch6 Ch7 Plotted: 16:02 Dec 11, 2000

< EMI - ElectroMagnetic Instruments 


\section{ROTATION ANGLE}

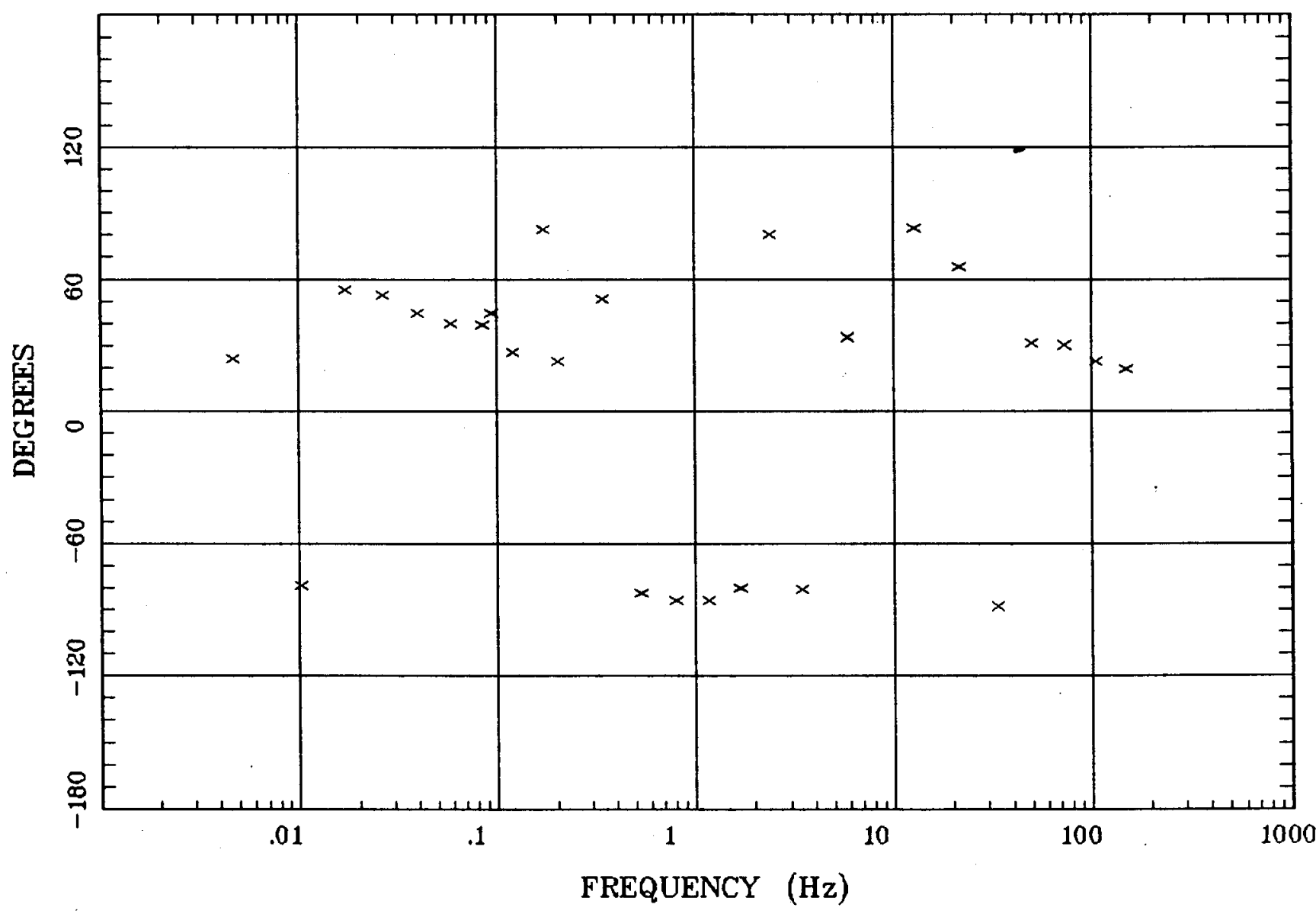

Client:

Remote:

Acquired:

Survey Co:
Rotation:

Filename: CT28C

Channels: Ch1 Ch2 Ch3 Ch4 Ch5 Ch6 Ch7

Plotted: 16:02 Dec 11, 2000

< EMI - ElectroMagnetic Instruments 
Station 28B

IMPEDANCE SKEW

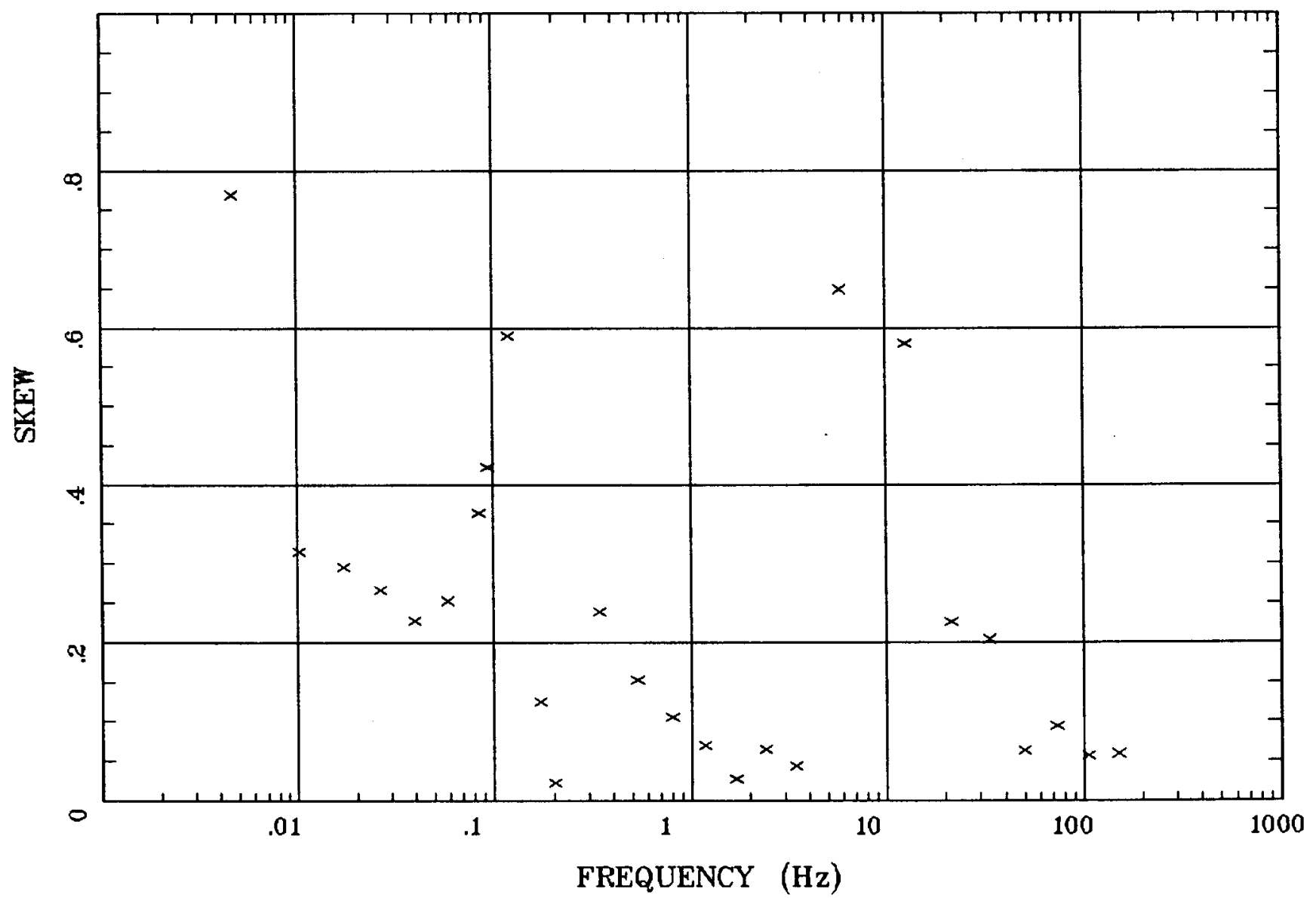

Client:

Rotation:

Remote:

Filename: CT28C

Channels: Ch1 Ch2 Ch3 Ch4 Ch5 Ch6 Ch7

Acquired:

Plotted:

16:02 Dec 11, 2000

Survey Co:

< EMI - ElectroMagnetic Instruments 
E MULT Coh.

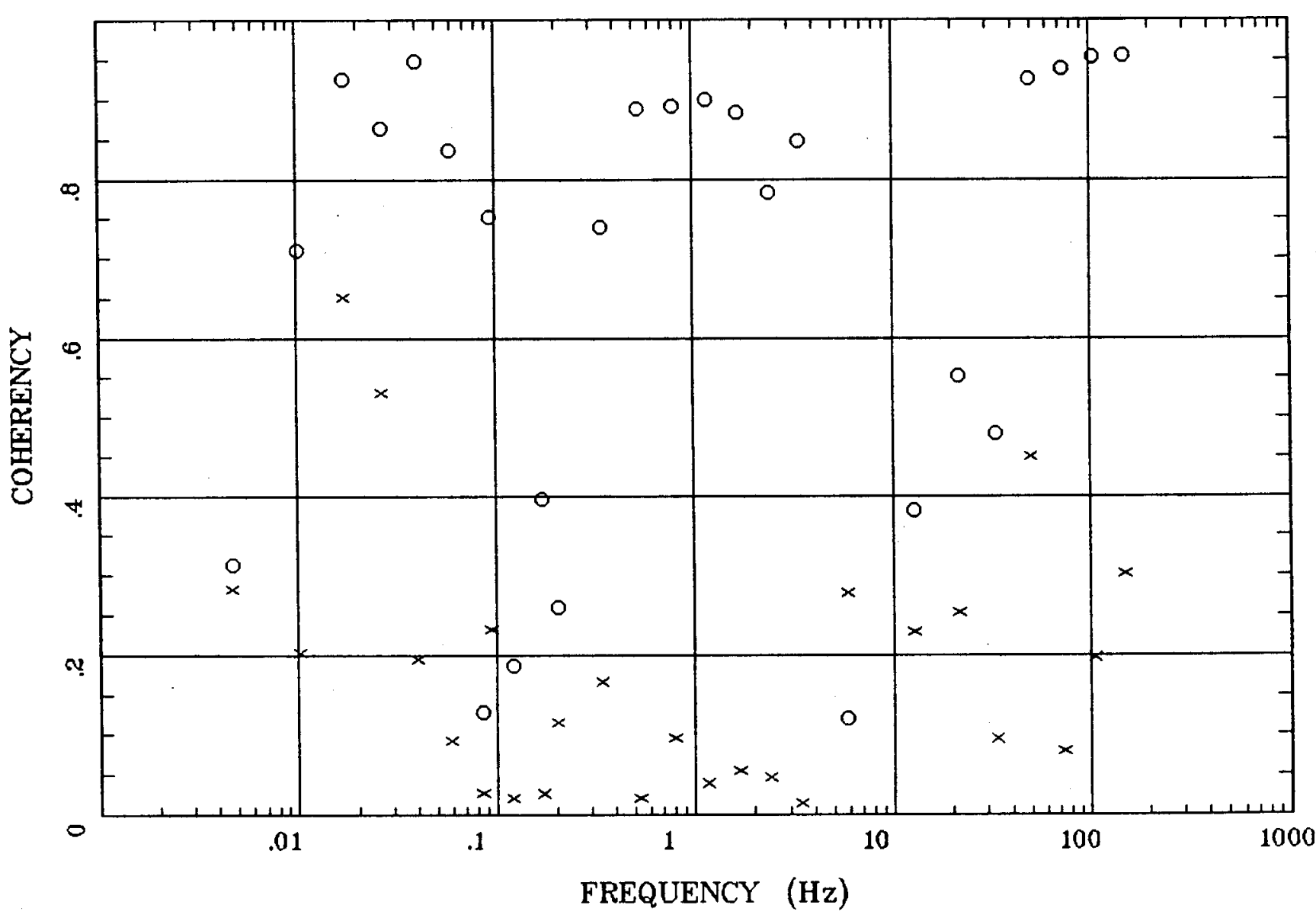

Client:

\section{Remote:}

Acquired:

Survey Co:
Rotation:

Filename: CT28C

Channels: Ch1 Ch2 Ch3 Ch4 Ch5 Ch6 Ch7

Plotted: 16:02 Dec 11, 2000

$<$ EMI - ElectroMagnetic Instruments > 
POLAR PLOTS

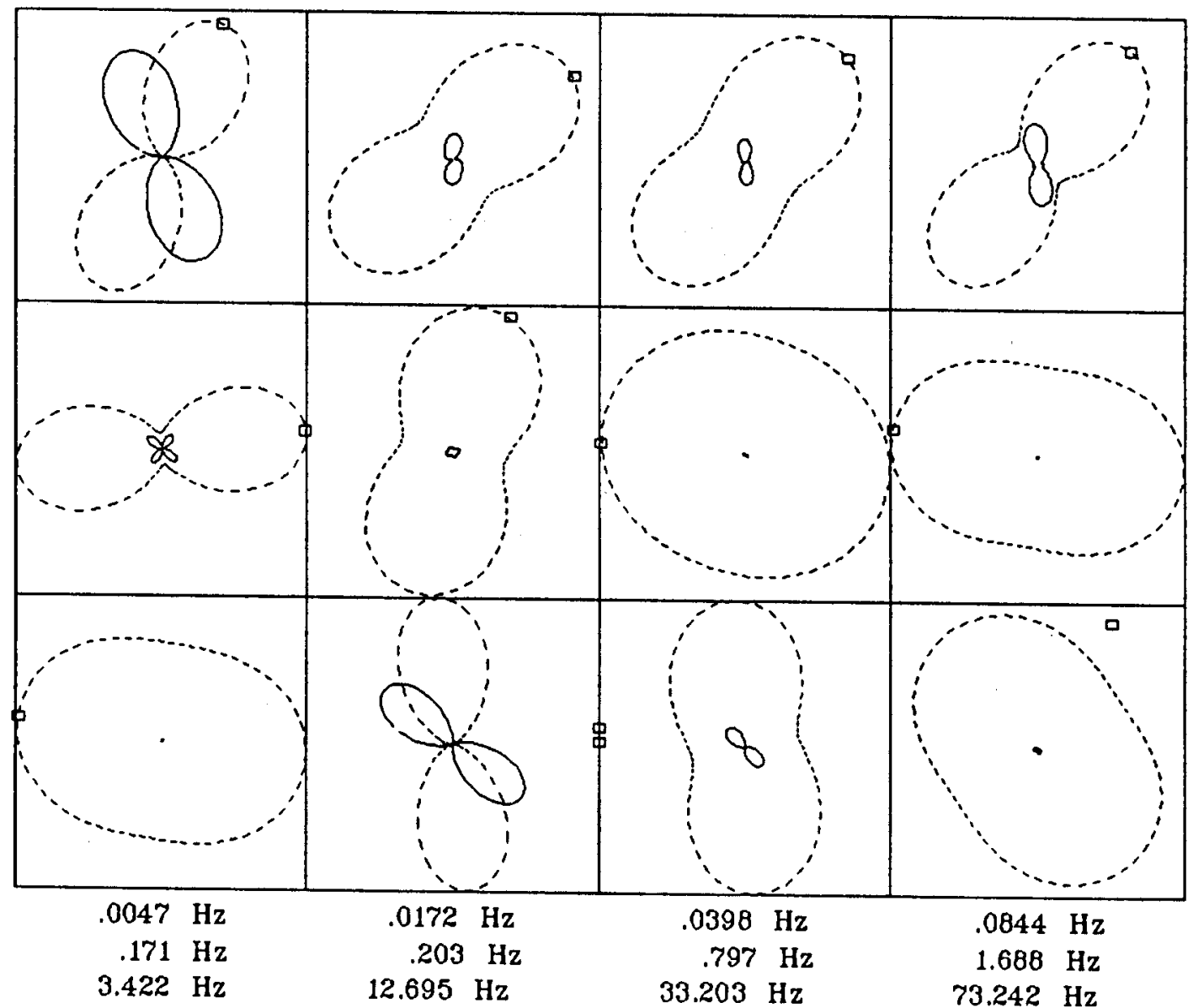

Client:

Remote:

Acquired:

Survey Co:
Rotation:

Filename: CT28C

Channels: Ch1 Ch2 Ch3 Ch4 Ch5 Ch6 Ch7 Plotted: 16:02 Dec 11, 2000

<EMI - ElectroMagnetic Instruments, 